\title{
High resolution CT imaging of finger joints
}

\author{
Citation for published version (APA):
}

Peters, MPH. (2018). High resolution CT imaging of finger joints: Automation of cortical interruption detection. [Doctoral Thesis, Maastricht University]. Maastricht University. https://doi.org/10.26481/dis.20180906mp

Document status and date:

Published: 01/01/2018

DOI:

10.26481/dis.20180906mp

Document Version:

Publisher's PDF, also known as Version of record

\section{Please check the document version of this publication:}

- A submitted manuscript is the version of the article upon submission and before peer-review. There can be important differences between the submitted version and the official published version of record.

People interested in the research are advised to contact the author for the final version of the publication, or visit the DOI to the publisher's website.

- The final author version and the galley proof are versions of the publication after peer review.

- The final published version features the final layout of the paper including the volume, issue and page numbers.

Link to publication

\footnotetext{
General rights rights.

- You may freely distribute the URL identifying the publication in the public portal. please follow below link for the End User Agreement:

www.umlib.nl/taverne-license

Take down policy

If you believe that this document breaches copyright please contact us at:

repository@maastrichtuniversity.nl

providing details and we will investigate your claim.
}

Copyright and moral rights for the publications made accessible in the public portal are retained by the authors and/or other copyright owners and it is a condition of accessing publications that users recognise and abide by the legal requirements associated with these

- Users may download and print one copy of any publication from the public portal for the purpose of private study or research.

- You may not further distribute the material or use it for any profit-making activity or commercial gain

If the publication is distributed under the terms of Article $25 \mathrm{fa}$ of the Dutch Copyright Act, indicated by the "Taverne" license above, 
High Resolution CT imaging of finger joints

Automation of cortical interruption detection 
(c) Michiel Peters, Maastricht, 2018

No part of this book may be reproduced or transmitted in any form or by any means, without prior permission in writing by the author, or when appropriate, by the publishers of the publications.

$\begin{array}{ll}\text { Cover: } & \text { Michiel Peters } \\ \text { Layout: } & \text { Tiny Wouters } \\ \text { Production: } & \text { Gildeprint B.V. }\end{array}$

ISBN/EAN: 978-94-9301-407-7

Printing of this thesis was financially supported by Maastricht University, Scanco Medical AG and the Dutch Arthritis Foundation 


\title{
High Resolution CT imaging of
}

\section{finger joints}

\author{
Automation of cortical interruption detection
}

\author{
PROEFSCHRIFT
}

ter verkrijging van de graad van doctor aan de Universiteit Maastricht, op gezag van de Rector Magnificus, Prof. dr. Rianne M. Letschert volgens het besluit van het College van Decanen,

in het openbaar te verdedigen op donderdag 6 september 2018, om 12:00 uur.

door

Michaël Petrus Henricus Peters 


\section{Promotoren}

Prof. dr. J.P.W. van den Bergh

Prof. dr. P.P.M.M. Geusens

\section{Co-promotoren}

Dr. A. van Tubergen

Dr. ir. B. van Rietbergen (Technische Universiteit Eindhoven)

\section{Beoordelingscommissie}

Prof. dr. A. Boonen (voorzitter)

Prof. dr. ir. W. Backes

Prof. dr. ir. K. Ito (Technische Universiteit Eindhoven)

Prof. dr. W. Lems (VU medisch centrum Amsterdam) 


\section{CONTENTS}

List of abbreviations

Chapter $1 \quad$ General introduction

Chapter 2 Visual detection of cortical breaks in hand joints: reliability and validity of high-resolution peripheral quantitative CT compared to microcT

BMC Musculoskeletal Disorders (2016)

Chapter 3 An automated algorithm for the detection of cortical interruptions on high resolution peripheral quantitative computed tomography images of finger joints Plos One (2017)

Chapter $4 \quad$ The reliability of a semi-automated algorithm for detection of cortical interruptions in finger joints on high resolution CT compared to microcT Calcified Tissue International (2017)

Chapter 5 An automated algorithm for the detection of cortical interruptions and its underlying loss of trabecular bone; a reproducibility study BMC Medical Imaging (2018)

Chapter 6 Assessment of cortical interruptions with semi-automated analysis of HR-pQCT in patients with rheumatoid arthritis versus healthy subjects and in comparison to radiography and MRI Journal of Bone Mineral Research (2018)

Chapter $7 \quad$ Longitudinal semi-automated quantification of cortical interruptions in finger joint using HR-pQCT in rheumatoid arthritis compared to healthy subjects

submitted

Chapter $8 \quad$ General discussion

Addendum Valorisation

Summary

Nederlandse samenvatting

Dankwoord

Curriculum Vitae

Publications 



\section{ABBREVIATIONS}

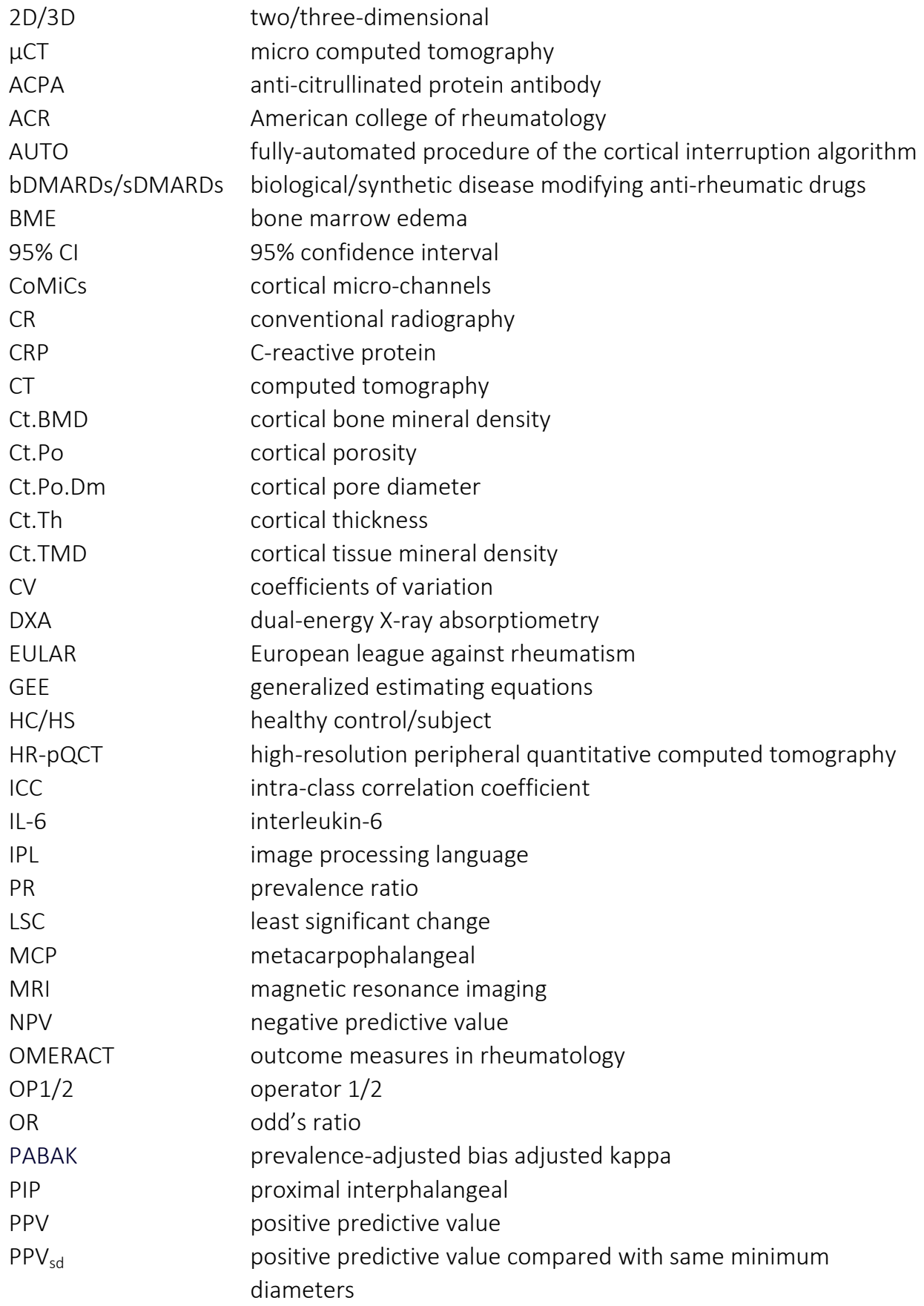


$P P V_{0.10 m m}$

PVE

RA

RANKL

RF

RMS

$\mathrm{ROI}$

SD

Sensitivity $_{s d}$

Sensitivity $0.10 \mathrm{~mm}$

SPECTRA

SvdH

Tb.BMD

Tb.N

Tb.Sp

Tb.SpSD

Tb.Th

TNFi

Tot.BMD

US

VBMD positive predictive value compared with fixed minimum diameter of $>0.10 \mathrm{~mm}$

partial volume effect

rheumatoid arthritis

receptor activator of nuclear factor kappa-B ligand

rheumatoid factor

root mean square

region of interest

standard deviation

sensitivity compared with same minimum diameters

sensitivity compared with fixed minimum diameter of $>0.10 \mathrm{~mm}$

Study grouP for xtrEme Computed Tomography in RA

Sharp/Van der Heijde

trabecular bone mineral density

trabecular number

trabecular separation

distribution of trabecular separation

trabecular thickness

tumor necrosis factor inhibitors

total bone mineral density

ultrasound

volumetric bone mineral density 


$$
1
$$



\begin{tabular}{l|l} 
& \\
General introduction & 11
\end{tabular}

\section{RHEUMATOID ARTHRITIS}

Approximately $1 \%$ of the population worldwide suffers from rheumatoid arthritis (RA), a chronic auto-immune inflammatory disease, that mainly affects the small joints of the hands, wrists and feet. ${ }^{1,2}$ RA is more common in females and symptoms consist primarily of pain, swelling and stiffness of the joints. Patients with RA are at risk for joint damage due to inflammation, which is characterized by cartilage damage leading to joint space narrowing and bone erosions. ${ }^{3}$ The presence of erosions is a predictor of further bone damage and is strongly associated with disability and reduced quality of life. ${ }^{4-7}$ Accurate and timely treatment is therefore important for the patients' well-being and the impact of the disease.

In the absence of adequate therapy, most patients with RA will develop bone damage visible by conventional radiography $(C R)^{8,9}$ Current treatment recommendations for RA support early and aggressive treatment to reduce the development of this bone damage.,10 Treatment with synthetic disease-modifying anti-rheumatic drugs (sDMARDs) and biologic drugs (bDMARDs) is directed at achieving symptom improvement, remission or low disease activity state, and inhibition of joint damage, the so called Treat-to-Target-strategy. ${ }^{11}$ Despite achieving remission, bone damage can still occur or progress. ${ }^{12}$

Both serological and imaging biomarkers can be used to identify those patients that are at risk for bone damage progression. Serological biomarkers that have been associated with risk for progression include rheumatoid factor (RF), ${ }^{13}$ anti-citrullinated protein antibodies $(\mathrm{ACPA})^{13-15}$ and $\mathrm{C}$-reactive protein $(\mathrm{CRP}){ }^{16}$ Imaging biomarkers allow visualization of inflammation and bone damage, which may not be determined by clinical examination. A variety of imaging techniques exists, each with their own particular advantages and disadvantages for use and interpretation in clinical practice.

\section{IMAGING IN RA}

Traditionally, imaging in RA is performed using $C R$, which is fast, inexpensive, easily accessible, provides a two-dimensional (2D) assessment of bone and rheumatologists are familiar with its interpretation. ${ }^{3}$ A major drawback of $C R$ is that it is not sensitive to detect erosions at an early stage and (subtle) progression over time. ${ }^{17}$ In addition, it cannot visualize inflammation of the joint. These limitations were the stimulus to apply other imaging modalities in RA, for example magnetic resonance imaging (MRI), ultrasound (US), and clinical Computed Tomography (CT).

MRI, with a resolution of $\sim 400 \mu \mathrm{m},{ }^{18}$ has the advantage of imaging the entire joint including surrounding soft tissues, the synovium and synovial fluid in three dimensions (3D). Therefore, MRI can not only detect bone erosions, but also quantify inflammation in the synovial fluid (synovitis) and in the bone marrow (osteitis/bone marrow edema $(\mathrm{BME})) .{ }^{19-22} \mathrm{MRI}$ is, however, expensive, time consuming, and its availability may be 
limited. Furthermore, erosions can be mimicked by lesions, such as focal bone edema or bone sclerosis, that do not involve bone loss, potentially leading to overestimation of the erosion size on MRI compared to clinical $\mathrm{CT}^{18,23}$

Musculoskeletal US is cheaper and less time consuming than MRI and allows detection of joint effusions, surrounding soft tissue involvement, and bone erosions, while power Doppler signal identifies the increased vascularity of inflamed tissues. ${ }^{24}$ Disadvantages of US are that it is operator dependent, it is not able to penetrate bone tissue for imaging, and standardization and validation are still incomplete. ${ }^{25}$

Clinical CT, with a resolution of $\sim 300 \mu \mathrm{m},{ }^{26}$ is currently the best modality to assess bone in 3D. ${ }^{23}$ Clinical CT has been proven to be superior in the detection of bone erosions compared to MRI and CR in patients with RA. ${ }^{18,23}$ Disadvantages of CT are its inability to visualize inflammation, its limited accessibility, and its high radiation exposure $(\sim 1 \mathrm{mSV}){ }^{26}$

\section{HIGH-RESOLUTION PERIPHERAL QUANTITATIVE CT IMAGING}

High-resolution peripheral quantitative CT (HR-pQCT) is a novel 3D imaging technique that allows in vivo evaluation of the cortical and trabecular bone micro-structure and volumetric bone mineral density (VBMD) of the peripheral skeleton, such as distal radius and tibia. ${ }^{27,28}$ The main advantage of HR-pQCT over clinical CT is its higher resolution (82 $\mathrm{mm}$ versus up to $300 \mu \mathrm{m}$ with MRI and clinical CT). ${ }^{18,26}$ This higher resolution enables capturing the bone micro-structure, also referred as "virtual bone biopsy". ${ }^{29}$ The assessment of the bone micro-structure on HR-pQCT is validated compared to microCT $(\mu \mathrm{CT})$ imaging, the 3D gold standard. ${ }^{27,30}$

The main disadvantages of HR-pQCT are the limited availability and experience, a longer scan time compared to clinical CT (typical scanning time is 3 minutes per $9 \mathrm{~mm}$ versus 1 second per $19 \mathrm{~mm}$, respectively ${ }^{31}$ ) which results in more frequently observed motion artefacts, $^{32,33}$ and the possibility of only scanning the peripheral skeleton. ${ }^{26}$

In the last decade, HR-pQCT imaging has been primarily used in studies on bone microstructure in metabolic bone diseases and osteoporosis in comparison to healthy subjects $^{28}$ and in intervention trials with anti-osteoporosis medication. ${ }^{34,35}$ More recently, HR-pQCT imaging has also been used to scan peri-articular bone in finger joints of patients with RA. ${ }^{36,37}$

\section{HR-pQCT imaging in RA}

The first studies with HR-pQCT in finger joints of patients with RA aimed to describe the potential of this imaging modality to detect peri-articular bone involvement in this population. Because of the high resolution, HR-pQCT imaging is more sensitive in the detection of structural bone damage in peri-articular bone of MCP and PIP joints in patients with RA compared to CR, MRI and US. ${ }^{37-41}$ Stach et al. showed that small 
\begin{tabular}{l|l} 
& \\
General introduction & 13
\end{tabular}

erosions $(<0.5 \mathrm{~mm}$ in diameter) could be detected on HR-pQCT images and that these erosions were found not only in patients with RA but also in healthy subjects. However, the number and size of erosions were greater in patients with RA, and erosions $>1.9 \mathrm{~mm}$ in diameter were considered highly specific for patients with RA. ${ }^{37}$ Fouque-Aubert et al. showed that patients with early RA (i.e. $<2$ years disease duration) without bone damage on $\mathrm{CR}$, had multiple erosions and a significantly impaired cortical volumetric bone density and thinner trabeculae on HR-pQCT compared to healthy subjects. ${ }^{36}$ Longitudinal studies using HR-pQCT imaging visualized partial repair of large erosions in patients with RA treated with tumor necrosis factor inhibitors (TNFi), interleukin-6 (IL-6) or receptor activator of nuclear factor kappa-B ligand (RANKL) blocker. ${ }^{42-44}$

In a previous study from our study group, we discovered that not only many cortical interruptions could be seen in both patients with RA and healthy subjects with HR-pQCT imaging, but that also a heterogeneous spectrum of cortical interruptions could be observed. ${ }^{45}$ Some (larger) cortical interruptions had underlying trabecular bone voids and were suggestive for an erosion, whereas other cortical interruptions were smaller and may represent physiological interruptions (i.e. vascular channels) (Figure 1.1). ${ }^{45}$
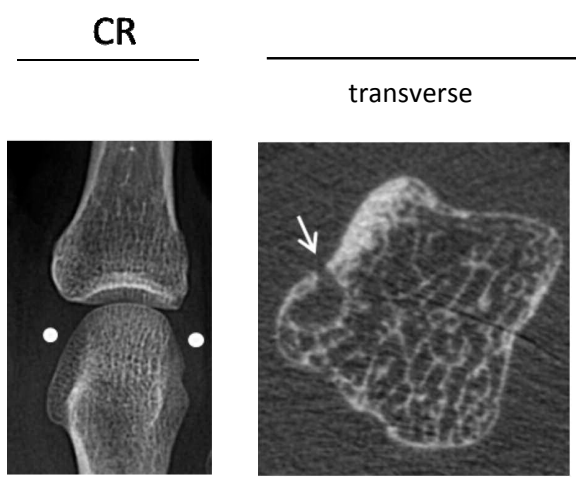

HR-pQCT

\section{a}
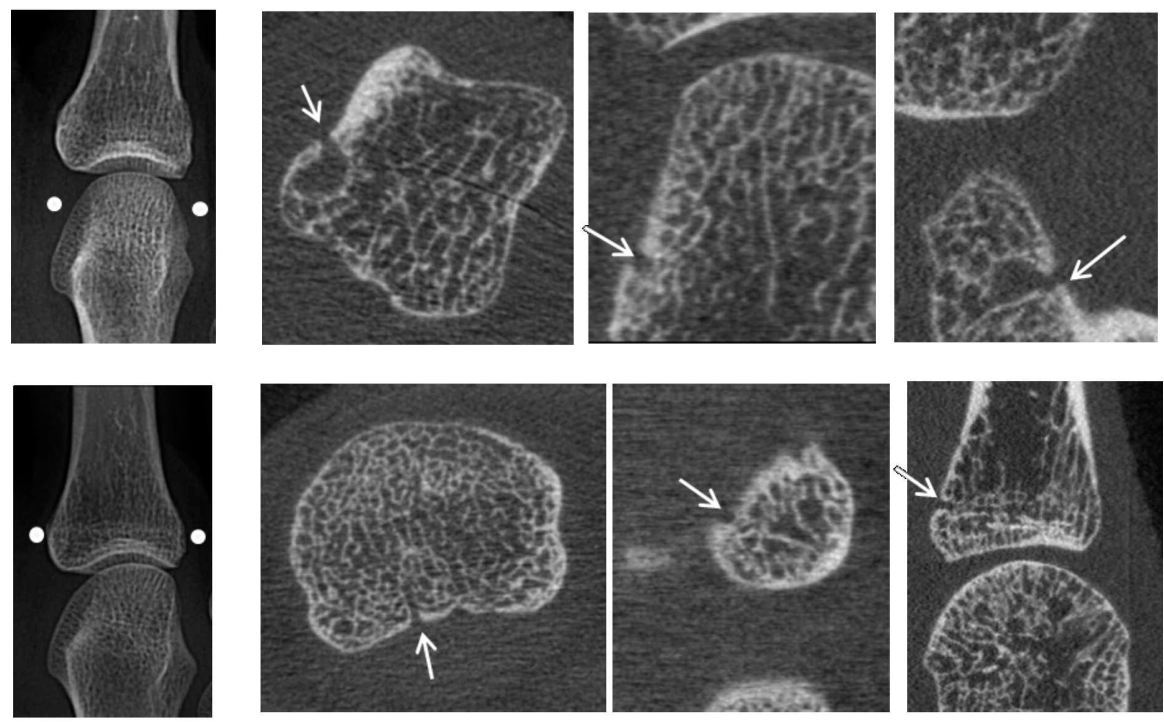

b

Figure 1.1. Normal MCP joints on CR, but cortical interruptions can be seen on HR-pQCT images with interpretations according to SPECTRA. (a.) Shows a cortical interruption which is suggestive for an erosion (adjacent trabecular bone loss). In (b.), a cortical interruption is seen on the palmar side (arrows), which is suggestive for a vascular channel (parallel lining). Image adapted from Scharmga and Peters et al. I Rheum $2016 .{ }^{45}$ 
$14 \mid$ Chapter 1

The Study grouP for xtrEme CT in RA (SPECTRA) proposed definitions to differentiate erosions from other cortical interruptions (i.e. vascular channels). ${ }^{46}$ They defined an erosion as an interruption in the cortical bone extending over a minimum of two consecutive slices in at least two perpendicular planes with adjacent trabecular bone loss (Figure 1.1a), and a vascular channel as an interruption in the cortical bone extending over a minimum of two consecutive slices in at least two perpendicular planes with parallel lining (Figure 1.1b). ${ }^{46}$ However, our study group showed that this preliminary definition for vascular channels was insufficient to capture most vascular channels using HR-pQCT. ${ }^{47}$

The detection of vascular channels in finger joints might be of importance in early diagnosis and monitoring of patients with RA. Vascular connections between bone marrow and the joint have been shown to be an anatomical location for bone damage in animal models of arthritis ${ }^{48,49}$ as they provide direct entry points for synovitis and osteoclast-mediated joint destruction. ${ }^{49,50}$ It has, therefore, been hypothesized that erosions might start in vascular channels. ${ }^{45,49-51}$

\section{Scoring of HR-pQCT images}

To date, cortical interruptions on HR-pQCT are evaluated by visual evaluation on multiple HR-pQCT slices (one scan stack with a length of $9 \mathrm{~mm}$ consists of 110 slices of $82 \mu \mathrm{m}) .{ }^{37-39,41-44,52} \mathrm{~A}$ major drawback of visual scoring is that it is not only prone to subjectivity, but also time consuming. Previous studies only scored erosions and did not incorporate all cortical interruptions (both erosions and small cortical interruptions). Earlier work from our study group, using visual scoring and incorporating all cortical interruptions, showed a substantial to almost perfect intra-reader reliability (ICC 0.69 and 0.88 ), but fair to moderate inter-reader reliability (ICC 0.37 and 0.56 ), and a poor feasibility (average scoring time per joint: $8.6 \mathrm{~min}$ (SD 3.7)) for application in clinical practice. $^{53}$

An automated algorithm for the detection of all cortical interruptions, could potentially improve reliability by minimizing reader interventions and improving feasibility in clinical practice. In one study, the development of an algorithm that accurately and reliably measured the volume of cortical interruptions has been described. ${ }^{54}$ However, only erosions were considered, an operator still needed to manually set a seeding point in the erosion, and manual corrections were necessary in the obtained volume in nearly half of the erosions. ${ }^{54}$ No fully-automated algorithm with inclusion of detection of small cortical interruptions was available.

\section{Bone mineral density and bone micro-structural parameters}

In addition to detection of (small) cortical interruptions, HR-pQCT imaging is also able to evaluate $V B M D$ and bone micro-structure in finger joints. ${ }^{36}$ Using the standard evaluation protocol, $V B M D$ is obtained in the total, trabecular and cortical region, and the bone micro-structure is captured in several micro-structural parameters (i.e. number 
and thickness of trabeculae (Tb.N and Tb.Th, respectively), separation and distribution of separation of trabeculae (Tb.Sp and Tb.SpSD, respectively), and thickness of the cortex (Ct.Th)). These morphometric measures are indicative of intra- and peri-articular bone loss which can also be observed in patients with RA (peri-articular osteopenia/ osteoporosis). ${ }^{51}$ Digital x-ray radiogrammetry (DXR) can measure BMD and cortical thickness in metacarpal bones. ${ }^{55}$ Previous studies showed that these parameters are correlated with functional status, disease activity parameters and erosive changes in $\mathrm{RA}^{55}$ and early loss of BMD on DXR predicted radiographic progression in RA. ${ }^{56}$

Using HR-pQCT imaging, a new method has been proposed to also determine cortical porosity (Ct.Po) and cortical tissue mineral density (Ct.TMD) in addition to VBMD and bone micro-structural parameters obtained from the standard evaluation protocol. ${ }^{57} \mathrm{~A}$ previous study evaluated the reproducibility of $\mathrm{VBMD}$ and bone micro-structural parameters in finger joints of patients with RA, but reproducibility of Ct.Po and Ct.TMD was not included. ${ }^{36}$ In addition, two cross-sectional studies evaluated VBMD and bone micro-structural parameters in finger joints of patients with RA in comparison to healthy subjects. $^{36,58}$ A lower $V B M D$ and impaired bone micro-structure in finger joints of patients with RA was found compared to healthy subjects, but Ct.Po and Ct.TMD were again not included. ${ }^{36,58}$ It is therefore unknown if Ct.Po and Ct.TMD are impaired in patients with RA compared to healthy subjects. Last, longitudinal studies evaluating change in vBMD and bone micro-structural parameters in patients with RA and healthy subjects are lacking.

\section{OBJECTIVES}

In this thesis, we describe the development of an algorithm for automated image analysis for detection of small cortical interruptions in finger joints, which aims at facilitating a more standardized and less time-consuming analysis of finger joints compared to visual scoring. When developing a new tool, it is important that it meets the requirements described in the Outcome Measures in Rheumatology (OMERACT, an independent initiative of health professionals interested in outcome measures in rheumatology): truth, discrimination and feasibility. ${ }^{59}$ By introducing this new automated algorithm, it is important to know whether this algorithm measures what is intended to measure (truth or validity), whether it discriminates between situations of interest (discrimination) and provides similar results on replication (reliability), and whether it is applicable in terms of time and interpretability (applicability).

In this thesis, reliability, validity and applicability of HR-pQCT in the detection of small cortical interruptions, bone density and bone micro-structure in finger joints of patients with RA and healthy subjects are studied. We compared the automated algorithm with the visual scoring method and with $\mu \mathrm{CT}$, the gold standard. Furthermore, we were specifically interested in the additional value of correction of contours by and operator ("semi-automated procedure") versus a fully automated procedure, to which extent the 
scores generated by the automated algorithm match between operators (inter-operator reliability) and on rescanning with in-between repositioning. We applied the automated method to patients with RA and healthy controls, and compared cortical interruptions, bone density and micro structural parameters between these groups and related findings on HR-pQCT to findings on MRI and CR both cross-sectionally and longitudinally.

\section{OUTLINE}

In Chapter 2, first, intra- and inter-reader reliability, and second, validity of visual detection of cortical interruptions using HR-pQCT compared to $\mu C T$ (as 3D gold standard) were studied using ten female index fingers from anatomic specimens from the Department of Anatomy and Embryology of the University of Amsterdam, the Netherlands. In Chapter 3, we introduced an automated algorithm for HR-pQCT images for detection of cortical interruptions, and studied the additional value of correction by an operator (semi-automated procedure) over a fully automated procedure. Subsequently, we compared the detection of cortical interruptions using the algorithm to visual scoring in a subset of patients with RA and healthy subjects from the MOSAHand cohort. This cohort consists of 38 female healthy subjects and 41 female patients diagnosed with RA from the Maastricht University Medical Center, the Netherlands. In Chapter 4, validity of the semi-automated algorithm for detection of different minimum cut-off diameters of cortical interruptions on HR-pQCT was studied and compared with $\mu \mathrm{CT}$, as gold standard, using ten female index fingers from anatomic specimens. In Chapter 5, we extended the semi-automated algorithm for detection of cortical interruptions with automated detection of underlying trabecular void volume, and studied the reproducibility of this extended algorithm in peri-articular bone of 17 early RA and four undifferentiated arthritis patients from the Early Inflammatory Arthritis Clinics of the Division of Rheumatology at the University of Calgary, Canada. Additionally, reproducibility of the peri-articular bone density and micro-structural parameters was studied in these subjects. In Chapter 6, the number and size of cortical interruptions, bone density and bone micro-structure were investigated in patients with RA and compared to healthy subjects from the MOSA-Hand cohort in a cross-sectional study. In addition, we investigated the association between structural damage on CR and MRI, and markers of inflammation on MRI with the number and size of cortical interruptions on HR-pQCT. In Chapter 7, change in cortical interruptions, bone density and bone micro-structure in MCP and PIP joints after one year follow-up was investigated in patients with RA and healthy subjects from the MOSA-Hand cohort. Finally, the overall findings of this thesis are summarized and discussed in Chapter 8. 


\section{REFERENCES}

1. Myasoedova E, Crowson CS, Kremers HM, Therneau TM, Gabriel SE. Is the incidence of rheumatoid arthritis rising?: results from Olmsted County, Minnesota, 1955-2007. Arthritis Rheum. 2010;62(6): 1576-82.

2. Alamanos Y, Voulgari PV, Drosos AA. Incidence and prevalence of rheumatoid arthritis, based on the 1987 American College of Rheumatology criteria: a systematic review. Semin Arthritis Rheum. 2006; 36(3):182-8.

3. van der Heijde DM. Radiographic imaging: the 'gold standard' for assessment of disease progression in rheumatoid arthritis. Rheumatology. 2000;39 Suppl 1:9-16.

4. van der Heijde D. Radiographic progression in rheumatoid arthritis: does it reflect outcome? Does it reflect treatment? Ann Rheum Dis. 2001;60 Suppl 3:iii47-50.

5. Clarke AE, St-Pierre Y, Joseph L, Penrod J, Sibley JT, Haga M, et al. Radiographic damage in rheumatoid arthritis correlates with functional disability but not direct medical costs. J Rheumatol. 2001;28(11): 2416-24.

6. Kirwan JR. Links between radiological change, disability, and pathology in rheumatoid arthritis. J Rheumatol. 2001;28(4):881-6.

7. Welsing PM, Landewe RB, van Riel PL, Boers M, van Gestel AM, van der Linden S, et al. The relationship between disease activity and radiologic progression in patients with rheumatoid arthritis: a longitudinal analysis. Arthritis Rheum. 2004;50(7):2082-93.

8. Lindqvist E, Jonsson K, Saxne T, Eberhardt K. Course of radiographic damage over 10 years in a cohort with early rheumatoid arthritis. Ann Rheum Dis. 2003;62(7):611-6.

9. Fuchs HA, Kaye JJ, Callahan LF, Nance EP, Pincus T. Evidence of significant radiographic damage in rheumatoid arthritis within the first 2 years of disease. J Rheumatol. 1989;16(5): 585-91.

10. Quinn MA, Green MJ, Conaghan P, Emery P. How do you diagnose rheumatoid arthritis early? Best Pract Res Clin Rheumatol. 2001;15(1):49-66.

11. Klarenbeek NB, Kerstens PJ, Huizinga TW, Dijkmans BA, Allaart CF. Recent advances in the management of rheumatoid arthritis. BMJ. 2010;341:c6942.

12. Hulsmans HM, Jacobs JW, van der Heijde DM, van Albada-Kuipers GA, Schenk Y, Bijlsma JW. The course of radiologic damage during the first six years of rheumatoid arthritis. Arthritis Rheum. 2000;43(9): 1927-40.

13. Ten Brinck RM, van Steenbergen HW, van Delft MAM, Verheul MK, Toes REM, Trouw LA, et al. The risk of individual autoantibodies, autoantibody combinations and levels for arthritis development in clinically suspect arthralgia. Rheumatology. 2017;56(12):2145-53.

14. Koga T, Okada A, Fukuda T, Hidaka T, Ishii T, Ueki Y, et al. Anti-citrullinated peptide antibodies are the strongest predictor of clinically relevant radiographic progression in rheumatoid arthritis patients achieving remission or low disease activity: A post hoc analysis of a nationwide cohort in Japan. PloS One. 2017;12(5):e0175281.

15. van der Helm-van Mil AH, Verpoort KN, Breedveld FC, Toes RE, Huizinga TW. Antibodies to citrullinated proteins and differences in clinical progression of rheumatoid arthritis. Arthritis Res Ther. 2005;7(5): R949-58.

16. Shimizu T, Choi HJ, Heilmeier U, Tanaka M, Burghardt AJ, Gong J, et al. Assessment of 3-month changes in bone microstructure under anti-TNFalpha therapy in patients with rheumatoid arthritis using highresolution peripheral quantitative computed tomography (HR-pQCT). Arthritis Res Ther. 2017;19(1):222.

17. Taouli B, Guermazi A, Sack KE, Genant HK. Imaging of the hand and wrist in RA. Ann Rheum Dis. 2002;61(10):867-9.

18. Dohn UM, Ejbjerg BJ, Hasselquist M, Narvestad E, Court-Payen M, Szkudlarek M, et al. Rheumatoid arthritis bone erosion volumes on $\mathrm{CT}$ and $\mathrm{MRI}$ : reliability and correlations with erosion scores on $\mathrm{CT}, \mathrm{MRI}$ and radiography. Ann Rheum Dis. 2007;66(10):1388-92.

19. Dohn UM, Ejbjerg BJ, Court-Payen M, Hasselquist M, Narvestad E, Szkudlarek M, et al. Are bone erosions detected by magnetic resonance imaging and ultrasonography true erosions? A comparison with computed tomography in rheumatoid arthritis metacarpophalangeal joints. Arthritis Res Ther. 2006; 8(4):R110. 
20. Conaghan PG, O'Connor P, McGonagle D, Astin P, Wakefield RJ, Gibbon WW, et al. Elucidation of the relationship between synovitis and bone damage: a randomized magnetic resonance imaging study of individual joints in patients with early rheumatoid arthritis. Arthritis Rheum. 2003;48(1):64-71.

21. Ostergaard M, Stoltenberg M, Lovgreen-Nielsen P, Volck B, Jensen CH, Lorenzen I. Magnetic resonance imaging-determined synovial membrane and joint effusion volumes in rheumatoid arthritis and osteoarthritis: comparison with the macroscopic and microscopic appearance of the synovium. Arthritis Rheum. 1997;40(10):1856-67.

22. Ostendorf B, Peters R, Dann P, Becker A, Scherer A, Wedekind F, et al. Magnetic resonance imaging and miniarthroscopy of metacarpophalangeal joints: sensitive detection of morphologic changes in rheumatoid arthritis. Arthritis Rheum. 2001;44(11):2492-502.

23. Perry D, Stewart N, Benton N, Robinson E, Yeoman S, Crabbe J, et al. Detection of erosions in the rheumatoid hand; a comparative study of multidetector computerized tomography versus magnetic resonance scanning. J Rheumatol. 2005;32(2):256-67.

24. Szkudlarek M, Klarlund M, Narvestad E, Court-Payen M, Strandberg C, Jensen KE, et al. Ultrasonography of the metacarpophalangeal and proximal interphalangeal joints in rheumatoid arthritis: a comparison with magnetic resonance imaging, conventional radiography and clinical examination. Arthritis Res Ther. 2006;8(2):R52.

25. Ostergaard M, Pedersen SJ, Dohn UM. Imaging in rheumatoid arthritis--status and recent advances for magnetic resonance imaging, ultrasonography, computed tomography and conventional radiography. Best Pract Res Clin Rheumatol. 2008;22(6):1019-44.

26. Burghardt AJ, Link TM, Majumdar S. High-resolution computed tomography for clinical imaging of bone microarchitecture. Clin Orthop Relat Res. 2011;469(8):2179-93

27. MacNeil JA, Boyd SK. Accuracy of high-resolution peripheral quantitative computed tomography for measurement of bone quality. Med Eng Phys. 2007;29(10):1096-105.

28. Boutroy S, Bouxsein ML, Munoz F, Delmas PD. In vivo assessment of trabecular bone microarchitecture by high-resolution peripheral quantitative computed tomography. J Clin Endocrinol Metab. 2005; 90(12):6508-15.

29. Nickolas TL, Shirazian S, Shane E. High-resolution computed tomography imaging: a virtual bone biopsy. Kidney Int. 2010;77(11):1046.

30. Krause M, Museyko O, Breer S, Wulff B, Duckstein C, Vettorazzi E, et al. Accuracy of trabecular structure by HR-pQCT compared to gold standard muCT in the radius and tibia of patients with osteoporosis and long-term bisphosphonate therapy. Osteoporos Int. 2014;25(5):1595-606.

31. Issever AS, Link TM, Kentenich M, Rogalla P, Burghardt AJ, Kazakia GJ, et al. Assessment of trabecular bone structure using MDCT: comparison of 64- and 320-slice CT using HR-pQCT as the reference standard. Eur Radiol. 2010;20(2):458-68.

32. Engelke K, Stampa B, Timm W, Dardzinski B, de Papp AE, Genant HK, et al. Short-term in vivo precision of $\mathrm{BMD}$ and parameters of trabecular architecture at the distal forearm and tibia. Osteoporos Int. 2012;23(8):2151-8.

33. Pialat JB, Burghardt AJ, Sode M, Link TM, Majumdar S. Visual grading of motion induced image degradation in high resolution peripheral computed tomography: impact of image quality on measures of bone density and micro-architecture. Bone. 2012;50(1):111-8.

34. Schafer AL, Burghardt AJ, Sellmeyer DE, Palermo L, Shoback DM, Majumdar S, et al. Postmenopausal women treated with combination parathyroid hormone (1-84) and ibandronate demonstrate different microstructural changes at the radius vs. tibia: the PTH and Ibandronate Combination Study (PICS). Osteoporos Int. 2013;24(10):2591-601.

35. Tsai JN, Uihlein AV, Burnett-Bowie SA, Neer RM, Zhu Y, Derrico N, et al. Comparative effects of teriparatide, denosumab, and combination therapy on peripheral compartmental bone density, microarchitecture, and estimated strength: the DATA-HRpQCT Study. J Bone Miner Res. 2015;30(1): 39-45.

36. Fouque-Aubert A, Boutroy S, Marotte H, Vilayphiou N, Bacchetta J, Miossec P, et al. Assessment of hand bone loss in rheumatoid arthritis by high-resolution peripheral quantitative CT. Ann Rheum Dis. 2010; 69(9):1671-6.

37. Stach CM, Bauerle M, Englbrecht M, Kronke G, Engelke K, Manger B, et al. Periarticular bone structure in rheumatoid arthritis patients and healthy individuals assessed by high-resolution computed tomography. Arthritis Rheum. 2010;62(2):330-9. 
38. Regensburger A, Rech J, Englbrecht M, Finzel S, Kraus S, Hecht K, et al. A comparative analysis of magnetic resonance imaging and high-resolution peripheral quantitative computed tomography of the hand for the detection of erosion repair in rheumatoid arthritis. Rheumatology (Oxford). 2015;54(9): 1573-81.

39. Lee CH, Srikhum W, Burghardt AJ, Virayavanich W, Imboden JB, Link TM, et al. Correlation of structural abnormalities of the wrist and metacarpophalangeal joints evaluated by high-resolution peripheral quantitative computed tomography, 3 Tesla magnetic resonance imaging and conventional radiographs in rheumatoid arthritis. Int J Rheum Dis. 2015;18(6):628-39.

40. Baillet A, Gaujoux-Viala C, Mouterde G, Pham T, Tebib J, Saraux A, et al. Comparison of the efficacy of sonography, magnetic resonance imaging and conventional radiography for the detection of bone erosions in rheumatoid arthritis patients: a systematic review and meta-analysis. Rheumatology. 2011; 50(6):1137-47.

41. Finzel S, Ohrndorf S, Englbrecht M, Stach C, Messerschmidt J, Schett G, et al. A detailed comparative study of high-resolution ultrasound and micro-computed tomography for detection of arthritic bone erosions. Arthritis Rheum. 2011;63(5):1231-6.

42. Finzel S, Rech J, Schmidt S, Engelke K, Englbrecht M, Stach C, et al. Repair of bone erosions in rheumatoid arthritis treated with tumour necrosis factor inhibitors is based on bone apposition at the base of the erosion. Ann Rheum Dis. 2011;70(9):1587-93.

43. Finzel S, Rech J, Schmidt S, Engelke K, Englbrecht M, Schett G. Interleukin-6 receptor blockade induces limited repair of bone erosions in rheumatoid arthritis: a micro CT study. Ann Rheum Dis. 2013; 72(3):396-400.

44. Yue J, Griffith JF, Xiao F, Shi L, Wang D, Shen J, et al. Repair of bone erosion in rheumatoid arthritis by denosumab: A high-resolution peripheral quantitative computed tomography study. Arthritis Care Res. 2017;69(8):1156-63.

45. Scharmga A, Peters M, van Tubergen A, van den Bergh J, Barnabe C, Finzel S, et al. Heterogeneity of Cortical Breaks in Hand Joints of Patients with rheumatoid arthritis and healthy controls imaged by highresolution peripheral quantitative computed tomography. J Rheumatol. 2016;43(10):1914-20.

46. Barnabe C, Toepfer D, Marotte H, Hauge EM, Scharmga A, Kocijan R, et al. Definition for rheumatoid arthritis erosions imaged with high resolution peripheral quantitative computed tomography and interreader reliability for detection and measurement. J Rheumatol. 2016; 43(10):1935-40.

47. Scharmga A, Keller KK, Peters M, van Tubergen A, van den Bergh JP, van Rietbergen B, et al. Vascular channels in metacarpophalangeal joints: a comparative histologic and high-resolution imaging study. Sci Rep. 2017;7(1):8966.

48. Benjamin M, Toumi H, Suzuki D, Redman S, Emery P, McGonagle D. Microdamage and altered vascularity at the enthesis-bone interface provides an anatomic explanation for bone involvement in the HLA-B27associated spondylarthritides and allied disorders. Arthritis Rheum. 2007;56(1):224-33.

49. Binks DA, Gravallese EM, Bergin D, Hodgson RJ, Tan AL, Matzelle MM, et al. Role of vascular channels as a novel mechanism for subchondral bone damage at cruciate ligament entheses in osteoarthritis and inflammatory arthritis. Ann Rheum Dis. 2015;74(1):196-203

50. Schett G, Stolina M, Bolon B, Middleton S, Adlam M, Brown H, et al. Analysis of the kinetics of osteoclastogenesis in arthritic rats. Arthritis Rheum. 2005;52(10):3192-201.

51. Schett G, Gravallese E. Bone erosion in rheumatoid arthritis: mechanisms, diagnosis and treatment. Nature reviews Rheumatology. 2012;8(11):656-64.

52. Srikhum W, Virayavanich W, Burghardt AJ, Yu A, Link TM, Imboden JB, et al. Quantitative and semiquantitative bone erosion assessment on high-resolution peripheral quantitative computed tomography in rheumatoid arthritis. J Rheumatol. 2013;40(4):408-16.

53. Scharmga A. High-resolution peripheral quantitative computed tomography in patients with rheumatoid arthritis: truth, discrimination and feasibility. Thesis UM 2017.

54. Topfer D, Finzel S, Museyko O, Schett G, Engelke K. Segmentation and quantification of bone erosions in high-resolution peripheral quantitative computed tomography datasets of the metacarpophalangeal joints of patients with rheumatoid arthritis. Rheumatology. 2014;53(1):65-71.

55. Pfeil A, Haugeberg G, Hansch A, Renz DM, Lehmann G, Malich A, et al. Value of digital X-ray radiogrammetry in the assessment of inflammatory bone loss in rheumatoid arthritis. Arthritis Care Res. 2011;63(5):666-74. 
56. Hoff M, Haugeberg G, Odegard S, Syversen S, Landewe R, van der Heijde D, et al. Cortical hand bone loss after 1 year in early rheumatoid arthritis predicts radiographic hand joint damage at 5-year and 10-year follow-up. Ann Rheum Dis. 2009;68(3):324-9.

57. Burghardt AJ, Buie HR, Laib A, Majumdar S, Boyd SK. Reproducibility of direct quantitative measures of cortical bone microarchitecture of the distal radius and tibia by HR-pQCT. Bone. 2010;47(3):519-28.

58. Yang H, Yu A, Burghardt AJ, Virayavanich W, Link TM, Imboden JB, et al. Quantitative characterization of metacarpal and radial bone in rheumatoid arthritis using high resolution- peripheral quantitative computed tomography. Int J Rheum Dis. 2017;20(3):353-62.

59. Boers M, Brooks P, Strand CV, Tugwell P. The OMERACT filter for outcome measures in rheumatology. J Rheumatol. 1998;25(2):198-9. 


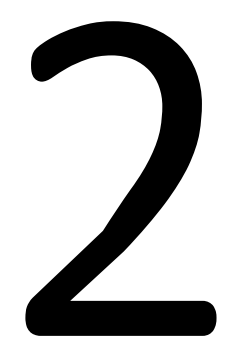

Visual detection of cortical breaks in hand joints: reliability and validity of high-resolution peripheral quantitative $\mathrm{CT}$ compared to microCT

A. Scharmga, M. Peters, A. van Tubergen, J. van den Bergh, J. de Jong, D. Loeffen, B. van Rietbergen, R. Weijers, P. Geusens 


\section{ABSTRACT}

\section{Background}

To study the reliability and validity of high-resolution peripheral quantitative CT (HR-pQCT) with microCT $(\mu \mathrm{CT})$ as gold standard in the visual detection of cortical breaks in metacarpophalangeal (MCP) and proximal interphalangeal (PIP) joints.

\section{Methods}

Ten cadaveric fingers (10 MCP and 9 PIP joints) were imaged by HR-pQCT and $\mu C T$ and visually analyzed by two independent readers. Intra- and inter-reader reliability were evaluated for the presence (yes/no, kappa statistics) and the total number (intra-class correlation coefficient, ICC) of cortical breaks. Sensitivity, specificity, positive and negative predictive value (PPV respectively NPV) of HR-pQCT in detecting cortical breaks were calculated.

\section{Results}

With HR-pQCT, mean 149 cortical breaks were identified and with $\mu \mathrm{CT}$ mean 129 $(p<0.05)$. Intra-reader reliability for the presence of a cortical break per quadrant was 0.52 (95\% Cl 0.48-0.56) and 0.71 (95\% Cl 0.67-0.75) for HR-pQCT and $\mu \mathrm{CT}$, respectively, and for the total number of cortical breaks $0.61(95 \% \mathrm{Cl} 0.49-0.70)$ and $0.75(95 \% \mathrm{Cl}$ $0.68-0.82)$. Inter-reader reliability for the presence of a cortical break per quadrant was 0.37 (95\% Cl 0.33-0.41) and 0.45 (95\% Cl 0.41-0.49) for HR-pQCT and $\mu \mathrm{CT}$, respectively, and for the number of cortical breaks $0.55(95 \% \mathrm{Cl} 0.43-0.65)$ and $0.54(95 \% \mathrm{Cl}$ 0.35-0.67). Sensitivity, specificity, PPV and NPV of HR-pQCT were $81.6 \%, 64.0 \%, 81.6 \%$, and $64.0 \%$ respectively.

\section{Conclusion}

Cortical breaks were commonly visualized in MCP and PIP joints with HR-pQCT and $\mu \mathrm{CT}$. Reliability of both HR-pQCT and $\mu C T$ was fair to moderate. HR-pQCT was highly sensitive to detect cortical breaks with $\mu \mathrm{CT}$ as gold standard. 
\begin{tabular}{l|l} 
& \multicolumn{2}{|l}{} \\
Visual detection of cortical breaks & 23
\end{tabular}

\section{INTRODUCTION}

Peri-articular cortical breaks are one of the characteristic features of bone involvement in rheumatoid arthritis (RA) and predictors of further radiographic progression. ${ }^{1,2}$ Early detection of cortical breaks is an important indicator for intensifying treatment in order to modify the disease course. ${ }^{3}$ In daily clinic, conventional radiographs (CR) are considered the gold standard for detection of cortical breaks in the hand joints in rheumatic diseases. CR is widely available, fast to perform, relatively cheap, and extensively validated, however its sensitivity to detect structural bone changes is low compared to computed tomography (CT), MRI and ultrasound. ${ }^{4-7}$ A novel, sensitive imaging technique is High-Resolution peripheral Quantitative Computed Tomography (HR-pQCT). ${ }^{8}$ HR-pQCT allows analysis of the cortical and trabecular microarchitecture of peripheral bones with an isotropic resolution of 82 micrometer $(\mu \mathrm{m})$. This technique is now also applied for 3-D assessment of the bone microarchitecture in the hand joints. ${ }^{8-10}$ A study by Stach et al. has demonstrated that HR-pQCT is more sensitive than CR in detecting cortical breaks in the hand joints in RA and also in healthy controls. ${ }^{8}$ However, the resolution of the HR-pQCT images can be of the same order as the thickness of the cortical bone in finger joints. Due to partial volume effects, it is possible that thin cortices are falsely identified as breaks. Therefore, in particular with thin cortices, the reliability, sensitivity and specificity of the measurements might be impaired and depend on the reader's perception.

The aims of this study were 1). to investigate the intra- and inter-reader reliability, and 2). to determine the sensitivity, specificity, positive predictive value (PPV) and negative predictive value (NPV) of HR-pQCT in detecting cortical breaks in hand joints, using $\mu \mathrm{CT}$ images with a much higher resolution $(18 \mu \mathrm{m})$ as gold standard. We were particularly interested in the methodology of identifying cortical breaks by HR-pQCT, not to study the clinical value of these cortical breaks. We hypothesized that HR-pQCT is a reliable and sensitive imaging method for identifying cortical breaks in hand joints compared to $\mu \mathrm{CT}$.

\section{METHODS}

\section{Specimens}

For this study, we used cadaveric specimen, because $\mu \mathrm{CT}$ imaging can only be executed in-vitro. MCP and PIP joints of ten female right hand human cadaveric index fingers were imaged by both HR-pQCT and $\mu \mathrm{CT}$. The donors had dedicated their body by testament signed during life to the Department of Anatomy and Embryology of the University of Amsterdam, the Netherlands. The fingers were fixated in formalin. 


\section{HR-pQCT and $\mu C T$ image acquisition}

HR-pQCT (XtremeCT1, Scanco Medical AG, Switzerland) scans were performed at clinical in vivo settings, i.e. at $60 \mathrm{kVp}$ tube voltage, $900 \mu \mathrm{A}$ tube current, $100 \mathrm{~ms}$ integration time and $82 \mu \mathrm{m}$ voxel size. $\mu \mathrm{CT}$ ( $\mu \mathrm{CT}$ 80, Scanco Medical AG, Switzerland) scans were performed at $70 \mathrm{kVp}$ tube voltage, $114 \mu \mathrm{A}$ tube current, $300 \mathrm{~ms}$ integration time and $18 \mu \mathrm{m}$ voxel size. On HR-pQCT, the region of interest of the MCP joint covered an area of $18.04 \mathrm{~mm}, 220$ slices and for the PIP joint $9.02 \mathrm{~mm}, 110$ slices.

On $\mu \mathrm{CT}$, the region of interest covered an area of $15.26 \mathrm{~mm}$; 848 slices and for the PIP joint 9.45 mm, 525 slices (Supplementary Figure S2.1).

\section{HR-pQCT and $\mu \mathrm{CT}$ image analysis}

Scans of HR-pQCT and $\mu \mathrm{CT}$ were exported in Digital Imaging and Communications in Medicine (DICOM) format and analyzed using Osirix (v.5.8.5 64-bit) multiplanar DICOM viewer. Differences in the extent of the scanned areas as well as in joint angles were noticed because the fingers were scanned horizontally on HR-pQCT and vertically on $\mu \mathrm{CT}$. Corresponding first and last slices of the overlapping region were visually determined to ensure that the same region of interest was used in the detection of cortical breaks on both imaging modalities.

Two trained readers (AS and MP) independently scored the HR-pQCT and $\mu C T$ images visually for the presence of cortical breaks. The readers received extensive training from Study grouP for xtrEme Computed Tomography in Rheumatoid Arthritis (SPECTRA) and have additional reading experience, before the current dataset was read. Readers were aware of the hypothesis of this study. The images were not anonymized. HR-pQCT images were first and independently scored from $\mu \mathrm{CT}$ images, with at least one day in between, and a two week interval for the rescoring by Reader 1 .

A cortical break was defined as a clear disruption of the cortex, seen on two consecutive slices on two orthogonal planes (on transverse and on sagittal or coronal plane) on HR-pQCT, and similarly, but on nine consecutive slices on $\mu C T$ to cover the same area as evaluated by HR-pQCT (Supplementary Figure S2.2).

To assess the location of the breaks in each joint, the transverse plane was divided into four quadrants: palmar, ulnar, dorsal and radial (Supplementary Figure S2.3). The phalangeal base and metacarpal head of the MCP joints, and proximal phalanx and distal phalanx of the PIP joints were separately assessed. In total eight quadrants per joint were analysed, four in the proximal bone, and four in the distal bone of the same joint. Each joint was systematically analysed per quadrant. Quadrants with large discrepancies between the readers (i.e. more than four breaks difference) were re-examined to identify reasons for discrepancy. Also, total volumetric bone mineral density (VBMD) of the specimens was calculated using HR-pQCT. 
Visual detection of cortical breaks $\mid 25$

\section{Statistics}

Descriptive analyses were done to calculate the total number of cortical breaks scored by the readers per quadrant for each imaging modality.

The difference in the total number of cortical breaks detected with HR-pQCT versus $\mu C T$ was tested for statistical significance with Wilcoxon signed-rank test. Intra- and inter-reader reliability were calculated using Cohen's Kappa ( $\mathrm{k}$ ) and intra-class correlations coefficient (ICC) with a two-way random model and absolute agreement. $\mathrm{k}$ value was calculated for the presence (yes/no) of a cortical break per quadrant and ICC values were calculated for the total number of cortical breaks per quadrant. $\mathrm{K}$ and ICC were calculated on the level of all available quadrants. Kappa values were also re-calculated, corrected for potential prevalence and bias within the kappa value (Prevalence-Adjusted Bias Adjusted Kappa, PABAK). ${ }^{11,12}$ Reliability was rated according to Landis et al.: $<0.00$ poor, 0.00-0.20 slight, 0.21-0.40 fair, 0.41-0.60 moderate, 0.61-0.80 substantial, 0.81-1.00 almost perfect. ${ }^{13}$ Sensitivity, specificity, PPV, and NPV of HR-pQCT in the detection of cortical breaks were calculated with $\mu \mathrm{CT}$ as gold standard. The mean value of the two readings of reader 1 (AS) was used for this purpose. Statistical analyses were performed with SPSS Statistics for Windows version 23.0 (IBM Corp., Armonk, NY).

\section{RESULTS}

The mean age of the donors was 85.1 (SD 9.6) years, the medical history was unknown. Average VBMD was $203 \mathrm{mgHA} / \mathrm{cm}^{3}$ for MCP joints, $293 \mathrm{mgHA} / \mathrm{cm}^{3}$ for PIP joints and $245 \mathrm{mgHA} / \mathrm{cm}^{3}$ for the total joints. The scans of ten MCP and nine PIP joints with in total 152 quadrants were available for analysis. One PIP joint could not be evaluated due to a missing $\mu \mathrm{CT}$ scan. Furthermore, Reader 1 considered the quality of the images of the metacarpal head in one MCP joint as too low on $\mu \mathrm{CT}$ due to a protocol error during scanning. Therefore four quadrants were excluded in the analyses of the $\mu \mathrm{CT}$ images.

Table 2.1 shows the total number of cortical breaks each reader found on HR-pQCT and $\mu C T$ images. The differences in scores between the first and second reading of Reader 1 were not statistically significant on HR-pQCT (139 vs. 118 breaks, $p=0.064)$ and $\mu$ CT (142 vs. 156 breaks, $p=0.163$ ). However, the difference in the mean score between HR-pQCT versus $\mu \mathrm{CT}$ was statistically significant (respectively, 129 and 149 breaks, $p<0.05$ ). The total number of cortical breaks on HR-pQCT scored by Reader 1 (first reading) versus Reader 2 was not statistically significant (respectively, 139 versus 151 breaks, $p=0.288$ ). On $\mu \mathrm{CT}$, Reader 2 found significantly more breaks than Reader 1 (first reading) (241 vs. 142 breaks, $p<0.001$ ). 
$26 \mid$ Chapter 2

Table 2.1. Number of cortical breaks scored per imaging modality

\begin{tabular}{lccr}
\hline & HR-pQCT & $\mu C T$ & $p$-value \\
\hline Reader 1 (first reading) & $139(7.3 \pm 4.1)$ & $142(7.4 \pm 4.0)$ & 0.562 \\
Reader 1 (second reading) & $118(6.2 \pm 2.6)$ & $156(8.2 \pm 3.6)$ & $<0.001$ \\
Mean score Reader 1 & $126(6.7 \pm 3.0)$ & $149(7.8 \pm 3.6)$ & 0.018 \\
Reader 2 & $151(7.9 \pm 3.3)$ & $241(12.6 \pm 6.3)$ & $<0.001$ \\
\hline
\end{tabular}

Total number of detected cortical breaks on the HR-pQCT and $\mu C T$ scans (mean \pm SD per joint). $N=19$ joints.

In total 4 quadrants with large discrepancies between the readers were re-examined. Several reasons for discrepancy were identified:

On $\mu \mathrm{CT}$ we defined a cortical break when present on nine consecutive slices. Sometimes Reader 1 observed the cortical break on eight consecutive slices, hence not considering it a break, whereas Reader 2 observed it on nine consecutive slices, thereby fulfilling the criteria for a break.

- $\quad$ The smaller the break, the less agreement between the readers. An example of this discrepancy is shown in Supplementary Figure S2.4a-b.

- Due to the low bone mineral density and thin cortices, there was low contrast in some cases (example in Supplementary Figure S2.4c).

- Reader 1 considered a break as one large break, whereas Reader 2 counted several small cortical breaks inside the same large break (example in Supplementary Figure S2.4d).

Table 2.2 shows the intra- and inter-reader reliability based on the 152 quadrants on HRpQCT and 148 quadrants on $\mu \mathrm{CT}$ that were evaluated. Intra-reader reliability was moderate to substantial for the presence of breaks (HR-pQCT: $K=0.52$ and $\mu C T$ : $K=0.71$ ) and for the number of breaks (HR-pQCT: ICC 0.61 and $\mu C T$ : ICC 0.75).

Inter-reader reliability was fair to moderate for the presence of breaks (HR-pQCT: $\mathrm{K}=0.37$ and $\mu \mathrm{CT}: \mathrm{K}=0.45$,) and for the number of breaks (HR-pQCT: ICC 0.55 and $\mu \mathrm{CT}$ : ICC 0.54). The values of PABAK were comparable (Table 2.2).

Table 2.2. Intra- and interreader reliability per imaging modality

\begin{tabular}{ccccccc}
\hline & \multicolumn{3}{c}{$\begin{array}{c}\text { Intra-reader } \\
\text { (reader 1) }\end{array}$} & \multicolumn{3}{c}{$\begin{array}{c}\text { Inter-reader } \\
\text { (reader 1 first reading versus reader 2 }\end{array}$} \\
\cline { 2 - 7 } & $\mathrm{K}$ & PABAK & $\mathrm{ICC}$ & $\mathrm{K}$ & PABAK & ICC \\
& $(95 \% \mathrm{Cl})$ & $(95 \% \mathrm{Cl})$ & $(95 \% \mathrm{Cl})$ & $(95 \% \mathrm{Cl})$ & $(95 \% \mathrm{Cl})$ & $(95 \% \mathrm{Cl})$ \\
\hline $\mathrm{HR}-$ pQCT & 0.52 & 0.53 & 0.61 & 0.37 & 0.38 & 0.55 \\
& $(0.48-0.56)$ & $(0.39-0.66)$ & $(0.49-0.70)$ & $(0.33-0.41)$ & $(0.23-0.53)$ & $(0.43-0.65)$ \\
$\mu \mathrm{CT}$ & 0.71 & 0.72 & 0.75 & 0.45 & 0.47 & 0.54 \\
& $(0.67-0.75)$ & $(0.60-0.83)$ & $(0.68-0.82)$ & $(0.41-0.49)$ & $(0.33-0.61)$ & $(0.35-0.67)$ \\
\hline
\end{tabular}

Intra- and inter-reader reliability of HR-pQCT and $\mu \mathrm{CT}$ images based on 152 quadrants for HR-pQCT and 148 quadrants on $\mu C T$. Kappa value was calculated on the presence of a cortical break (yes/no) and ICC was calculated on the total number of cortical breaks in all quadrants

Sensitivity, specificity, PPV and NPV of HR-pQCT in the detection of cortical breaks with $\mu \mathrm{CT}$ as gold standard were calculated with the mean scores of Reader 1 (reading 1 and 
2) and the score of Reader 2 . The sensitivity was $81.6 \%$, specificity $64 \%$, PPV $81.6 \%$, and NPV 64\% respectively for Reader 1 , and sensitivity $68.9 \%$, specificity $69.4 \%$, PPV $82.6 \%$ and NPV 51.5\% for Reader 2 (Table 2.3 and Supplementary Tables S2.1-S2.6).

Table 2.3. Sensitivity, specificity, positive predictive value and negative predictive value of HR-pQCT

\begin{tabular}{lcccccc}
\hline Joints & $\begin{array}{c}\text { Total number of } \\
\text { breaks on HR- } \\
\text { pQCT }\end{array}$ & $\begin{array}{c}\text { Total number } \\
\text { of breaks on } \\
\mu C T\end{array}$ & Sensitivity & Specificity & $\begin{array}{c}\text { Positive } \\
\text { predictive } \\
\text { value }\end{array}$ & $\begin{array}{c}\text { Negative } \\
\text { predictive } \\
\text { value }\end{array}$ \\
\hline MCP reader 1 & 68 & 79 & $82.1 \%$ & $65.0 \%$ & $86.8 \%$ & $56.5 \%$ \\
MCP reader 2 & 83 & 156 & $65.6 \%$ & $62.5 \%$ & $87.5 \%$ & $31.3 \%$ \\
PIP reader 1 & 61 & 70 & $81.0 \%$ & $63.3 \%$ & $75.6 \%$ & $70.4 \%$ \\
PIP reader 2 & 68 & 85 & $75.6 \%$ & $77.4 \%$ & $81.6 \%$ & $70.6 \%$ \\
All reader 1 & 129 & 149 & $81.6 \%$ & $64.0 \%$ & $81.6 \%$ & $64.0 \%$ \\
All reader 2 & 151 & 241 & $68.9 \%$ & $69.4 \%$ & $82.6 \%$ & $51.5 \%$ \\
\hline
\end{tabular}

Sensitivity, specificity, positive predictive value and negative predictive value of HR-pQCT in the detection of cortical breaks with $\mu \mathrm{CT}$ as gold standard based on the mean score of Reader 1 (readings 1 and 2), and the score of Reader 2.

In Figure 2.1, several examples of cortical breaks on corresponding HR-pQCT and $\mu \mathrm{CT}$ images are presented. Figure 2.1a-b show a cortical break on both HR-pQCT and $\mu \mathrm{CT}$. Figure 2.1c, a discontinuity of the cortex is found on HR-pQCT. However, it did not meet the definition of a cortical break applied in this study, because it was visible on one slice only, leading to discrepancy with the results from $\mu \mathrm{CT}$. Figure $2.1 d$, a cortical break was detected on HR-pQCT, but not on $\mu \mathrm{CT}$, where a thin cortical lining was seen.

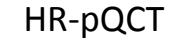

a.

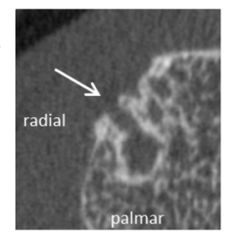

b.

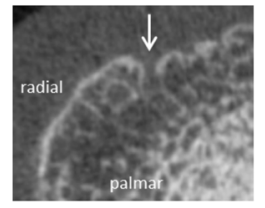

$\mu \mathrm{CT}$
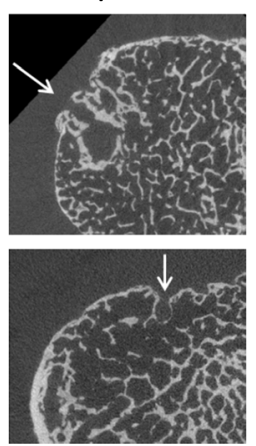

HR-pQCT

C.

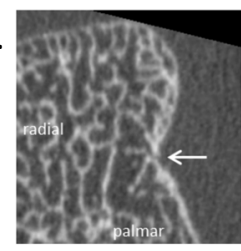

d.

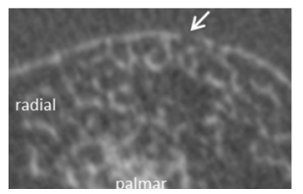

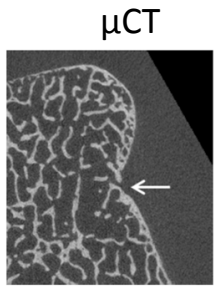

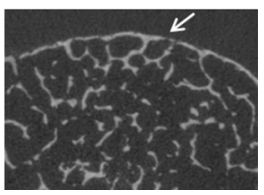

Figure 2.1. Corresponding images of cortical breaks on HR-pQCT and $\mu \mathrm{CT}$. (а.) A discontinuity of the cortex (arrow) meeting the definition of a cortical break was seen on both HR-pQCT and $\mu \mathrm{CT}$. (b.) A discontinuity of the cortex (arrow) meeting the definition of a cortical break was seen on both HR-pQCT and $\mu C T$. (c.) A discontinuity of the cortex (arrow) is seen on one slice only on HR- PQCT, thereby not fulfilling the definition for a break on HR-pQCT. A clear cortical break (arrow) is seen on $\mu \mathrm{CT}$, which was also seen on nine subsequent slices, thereby fulfilling the definition of a break. (d.) A discontinuity of the cortex (arrow) meeting the definition of a cortical break is seen on HR-pQCT. On $\mu \mathrm{CT}$, the cortical lining is intact (arrow). MCP; metacarpophalangeal, HR-pQCT; high-resolution peripheral quantitative computed tomography, $\mu \mathrm{CT}$; microCT. 


\section{DISCUSSION}

This study is the first that reports on aspects of reliability and validity of detecting cortical breaks in hand joints using HR-pQCT with $\mu \mathrm{CT}$ as gold standard. Cortical breaks were found in all joints with both imaging modalities. Intra-reader reliability of HR-pQCT and $\mu \mathrm{CT}$ was moderate to substantial, while inter-reader reliability was fair to moderate. The sensitivity of HR-pQCT in detecting cortical breaks was high (81.6\%).

In our study, only cortical discontinuities meeting the definition of a break, i.e. clearly visible cortical interruptions on at least $2 \mathrm{HR}$-pQCT (or 9 on $\mu \mathrm{CT}$ ) consecutive slices in 2 planes, were scored as a cortical break. They may have different pathological or physiological backgrounds, such as erosions or vascular channels. ${ }^{8,9}$ A formal classification system for defining breaks visualized on HR-pQCT and $\mu C T$ is lacking. Histological examination is needed to provide more insight in the nature of these cortical breaks and for developing definitions.

In a previous study by Stach et al. an almost perfect and substantial intra- and inter-reader reliability was reported using HR-pQCT for grading bone lesions and discrimination between healthy individuals and RA patients ( $\mathrm{k}=0.82$ and $\mathrm{k}=0.75$ respectively), but reliability on the presence and number of cortical breaks was not reported. ${ }^{8}$ The precision in scoring abnormalities visualized with several imaging techniques varies widely, even by experienced readers, as has been demonstrated for example for scoring radiographs in RA (ICC ranged from 0.65 to 0.99). ${ }^{14}$ In general, lower values for inter-reader reliability in comparison with intra-reader reliability are reported, ${ }^{15}$ corresponding to our findings. In our study, the breaks were scored visually, which is reader dependent. An automatic scoring algorithm, with detection of predefined definitions of breaks and executed automatically by the computer, could potentially improve reliability by minimizing reader interventions.

We investigated the sensitivity of HR-pQCT in detecting cortical breaks with $\mu \mathrm{CT}$ as the gold standard and found a high sensitivity (81.6\%). Unfortunately, no comparative studies are available. In contrast, two studies used HR-pQCT as the reference method for investigating the sensitivity to detect cortical breaks of other imaging modalities. ${ }^{9,16}$ These studies reported a sensitivity of $85.7 \%$ for MRI, $60.9 \%$ for CR, and $83-100 \%$ for ultrasound with HR-pQCT as the reference method. ${ }^{9,16}$ We found a lower specificity of HR-pQCT in detecting cortical breaks (64\%) in comparison to sensitivity. A possible explanation for this could be a phenomenon attributed to a partial volume effect leading to a reduced cortical signal on HR-pQCT, giving the impression that a cortical break is present, whereas on $\mu \mathrm{CT}$ the cortex is intact. An example of this is shown in Figure 2.1d. There are several limitations of this study. First, we evaluated whether the total number of breaks counted per quadrant corresponded between the two imaging modalities, but did not consider correspondence in exactly the same location. This might have led to an overestimation of the reliability. Second, we used fingers from cadaver specimens with unknown medical history and a relatively high mean age (85.1 years). Due to the old age of the donors, and the preservation in formalin, the cortices might become less 
mineralized. ${ }^{17}$ The average vBMD of the specimens was $245 \mathrm{mgHA} / \mathrm{cm}^{3}$, which is some $20 \%$ lower than the average in the normal population $\left(>300 \mathrm{mgHA} / \mathrm{cm}^{3}\right) .{ }^{10,18}$ This may hamper the scoring of a cortical break on HR-pQCT. It is also possible that thin regions were falsely identified as a cortical break. However, the use of cadaveric specimens was essential as in vivo human subjects cannot be measured by $\mu \mathrm{CT}$ because of a long scanning time. Third, the cadaveric specimens had slightly different orientations in the HR-pQCT versus the $\mu \mathrm{CT}$ scanner. Despite the careful visual matching of the regions of interest on HR-pQCT and $\mu \mathrm{CT}$, the angle at which the transversal images were viewed was slightly different in some joints and a cortical break might therefore be missed.

Fourth, a discrepancy between the readers regarding the number of cortical breaks identified on $\mu \mathrm{CT}$ was noticed. $\mu \mathrm{CT}$ images provide much detail, and in particular very small cortical interruptions were not always picked up by Reader 1 . This indicates that, when visually analyzing $\mu \mathrm{CT}$ images, more stringent definitions are necessary than when using HR-pQCT because of the higher resolution.

\section{CONCLUSIONS}

Cortical breaks were commonly visualized in hand joints with HR-pQCT and $\mu C T$. Reliability of both HR-pQCT and $\mu \mathrm{CT}$ was fair to moderate. HR-pQCT was sensitive to detect cortical breaks with $\mu \mathrm{CT}$ as gold standard. In spite of the limitations of our study, including the discrepancy of $\mu \mathrm{CT}$ results between the readers, we have shown that HR-pQCT is highly sensitive to detect cortical breaks with a fair to moderate reliability compared to $\mu \mathrm{CT}$. Our findings need further evaluation, preferably with focus on histological analyses to clarify the nature of the breaks and to establish more reliable definitions and a classification system for analyzing cortical breaks on high-resolution CT images. 


\section{REFERENCES}

1. Schett G, Gravallese E. Bone erosion in rheumatoid arthritis: mechanisms, diagnosis and treatment. Nat Rev Rheumatol. 2012;8(11):656-64.

2. Geusens P, van den Bergh J. Bone erosions in rheumatoid arthritis. Rheumatology (Oxford). 2014;53(1):4-5.

3. Sommer OJ, Kladosek A, Weiler V, Czembirek H, Boeck M, Stiskal M. Rheumatoid arthritis: a practical guide to state-of-the-art imaging, image interpretation, and clinical implications. Radiographics. 2005;25(2):381-98.

4. Baillet A, Gaujoux-Viala C, Mouterde G, Pham T, Tebib J, Saraux A, et al. Comparison of the efficacy of sonography, magnetic resonance imaging and conventional radiography for the detection of bone erosions in rheumatoid arthritis patients: a systematic review and meta-analysis. Rheumatology (Oxford). 2011;50(6):1137-47.

5. Geusens P, Chapurlat R, Schett G, Ghasem-Zadeh A, Seeman E, de Jong J, et al. High-resolution in vivo imaging of bone and joints: a window to microarchitecture. Nat Rev Rheumatol. 2014;10(5):304-13.

6. Saraux A, Berthelot JM, Chales G, Le Henaff C, Thorel JB, Hoang S, et al. Ability of the American College of Rheumatology 1987 criteria to predict rheumatoid arthritis in patients with early arthritis and classification of these patients two years later. Arthritis Rheum. 2001;44(11):2485-91.

7. Dohn UM, Ejbjerg BJ, Hasselquist M, Narvestad E, Moller J, Thomsen HS, et al. Detection of bone erosions in rheumatoid arthritis wrist joints with magnetic resonance imaging, computed tomography and radiography. Arthritis Res Ther. 2008;10(1):R25.

8. Stach CM, Bauerle M, Englbrecht M, Kronke G, Engelke K, Manger B, et al. Periarticular bone structure in rheumatoid arthritis patients and healthy individuals assessed by high-resolution computed tomography. Arthritis Rheum. 2010;62(2):330-9.

9. Finzel S, Ohrndorf S, Englbrecht M, Stach C, Messerschmidt J, Schett G, et al. A detailed comparative study of high-resolution ultrasound and micro-computed tomography for detection of arthritic bone erosions. Arthritis Rheum. 2011;63(5):1231-6.

10. Fouque-Aubert A, Boutroy S, Marotte H, Vilayphiou N, Bacchetta J, Miossec P, et al. Assessment of hand bone loss in rheumatoid arthritis by high-resolution peripheral quantitative CT. Ann Rheum Dis. 2010;69(9):1671-6.

11. Sim J, Wright CC. The kappa statistic in reliability studies: use, interpretation, and sample size requirements. Phys Ther. 2005;85(3):257-68.

12. Byrt T, Bishop J, Carlin JB. Bias, prevalence and kappa. J Clin Epidemiol. 1993;46(5):423-9.

13. Landis JR, Koch GG. The measurement of observer agreement for categorical data. Biometrics. 1977;33(1):159-74.

14. Sharp JT, Wolfe F, Lassere M, Boers M, Van Der Heijde D, Larsen A, et al. Variability of precision in scoring radiographic abnormalities in rheumatoid arthritis by experienced readers. J Rheumatol. 2004;31(6):1062-72.

15. Salaffi F, Carotti M. Interobserver variation in quantitative analysis of hand radiographs in rheumatoid arthritis: comparison of 3 different reading procedures. J Rheumatol. 1997;24(10):2055-6.

16. Lee $\mathrm{CH}$, Srikhum W, Burghardt AJ, Virayavanich W, Imboden JB, Link TM, et al. Correlation of structural abnormalities of the wrist and metacarpophalangeal joints evaluated by high-resolution peripheral quantitative computed tomography, 3 Tesla magnetic resonance imaging and conventional radiographs in rheumatoid arthritis. Int J Rheum Dis. 2015;18(6):628-39.

17. Zebaze RM, Ghasem-Zadeh A, Bohte A, Iuliano-Burns S, Mirams M, Price RI, et al. Intracortical remodelling and porosity in the distal radius and post-mortem femurs of women: a cross-sectional study. Lancet. 2010;375(9727):1729-36.

18. Feehan L, Buie H, Li L, McKay H. A customized protocol to assess bone quality in the metacarpal head, metacarpal shaft and distal radius: a high resolution peripheral quantitative computed tomography precision study. BMC Musculoskelet Disord. 2013;14:367. 


\section{SUPPLEMENTARY FILES}

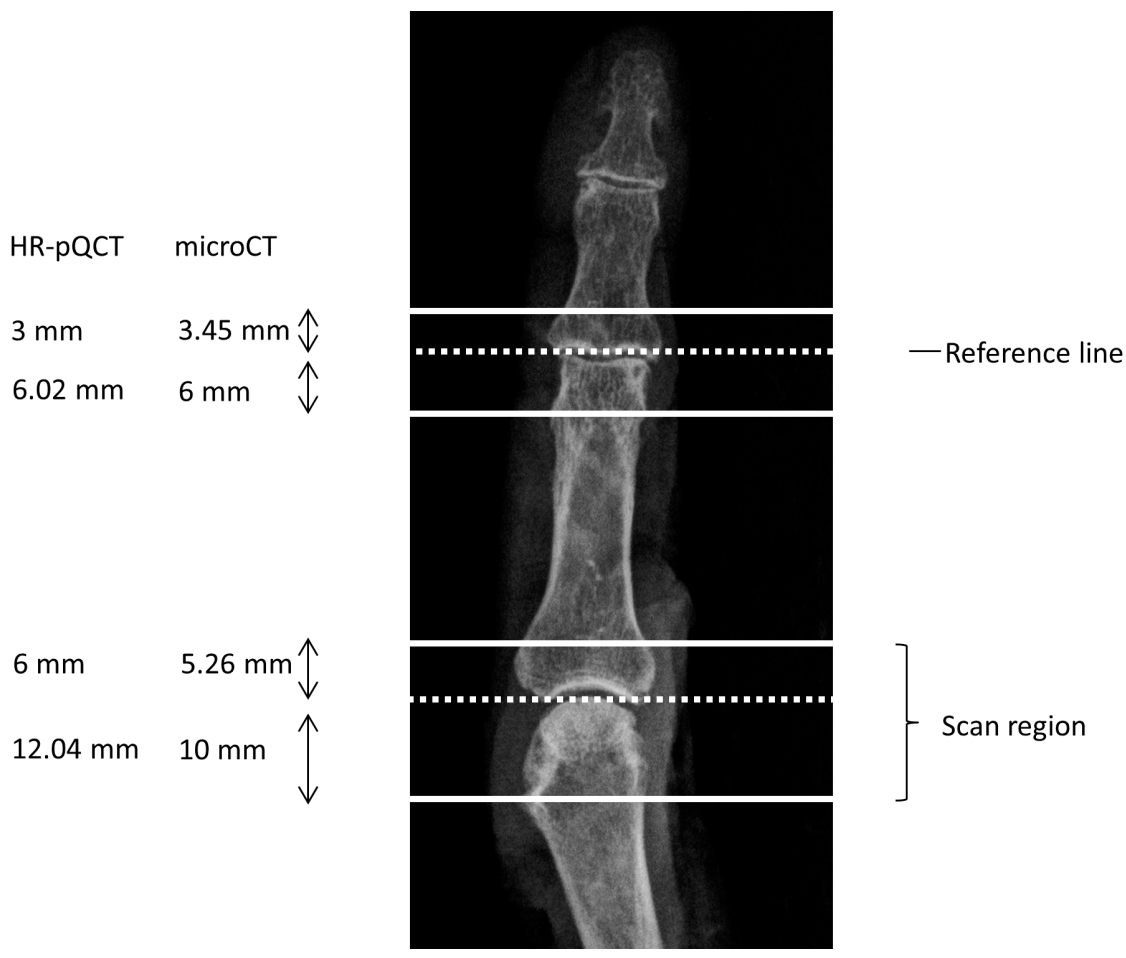

Figure S2.1 Method of selection of regions of interest. Method of selection of regions of interest in an MCP and PIP joint on HR-pQCT and $\mu C T$. Total scan area for an MCP joint on HR-pQCT was $18.04 \mathrm{~mm}$ and for a PIP joint $9.02 \mathrm{~mm}$. Total scan area for an MCP joint on $\mu \mathrm{CT}$ was $15.26 \mathrm{~mm}$ and for a PIP joint $9.45 \mathrm{~mm}$. HR-pQCT; highresolution peripheral quantitative computed tomography, $\mu \mathrm{CT}$; micro computed tomography, $\mathrm{MCP}$; metacarpophalangeal, PIP; proximal interphalangeal.

\section{HR-pQCT $82 \mu \mathrm{m}$ per slice}
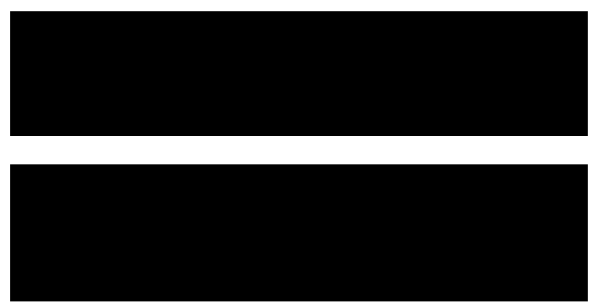

$\mu \mathrm{CT} 18 \mu \mathrm{m}$ per slice

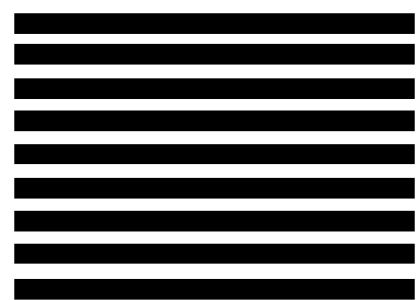

Figure S2.2. Resolution of HR-pQCT and $\mu \mathrm{CT}$ imaging. Resolution of HR-pQCT imaging is $82 \mu \mathrm{m}$, while the resolution of $\mu \mathrm{CT}$ imaging is $18 \mu \mathrm{m}$. To match both resolutions, two consecutive slices on HR-pQCT correspond with 9 consecutive slices on $\mu \mathrm{CT}$. HR-pQCT; high-resolution peripheral quantitative computed tomography, $\mu \mathrm{CT}$; microCT. 


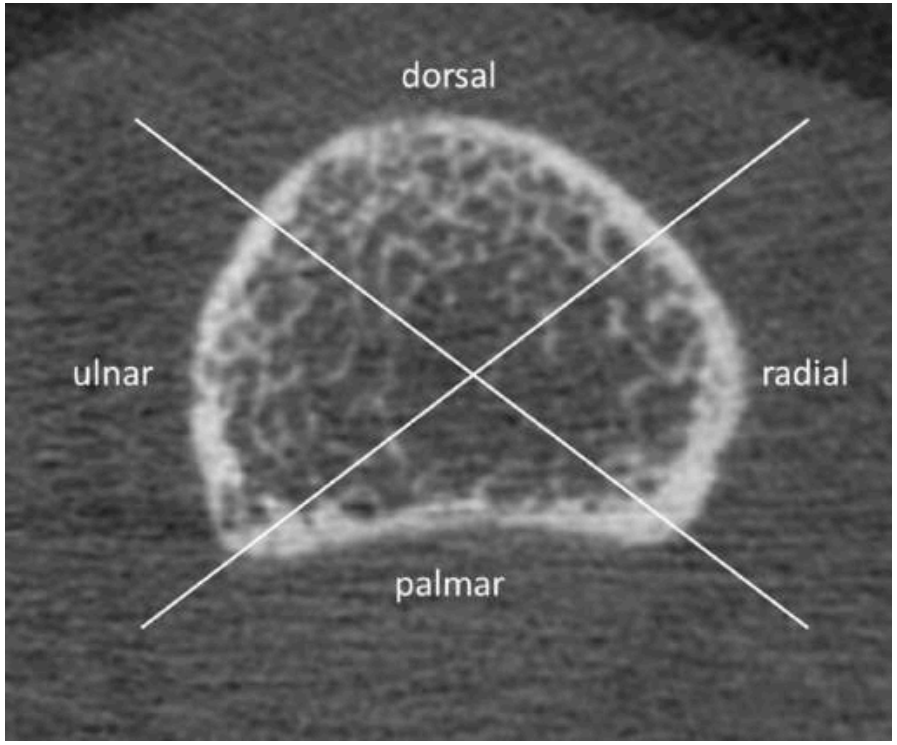

Figure S2.3. Division of a phalangeal base of an MCP joint. Phalangeal base of an MCP joint divided into palmar, ulnar, dorsal and radial quadrants. MCP; metacarpophalangeal.

\section{$\mu \mathrm{CT}$}

a.

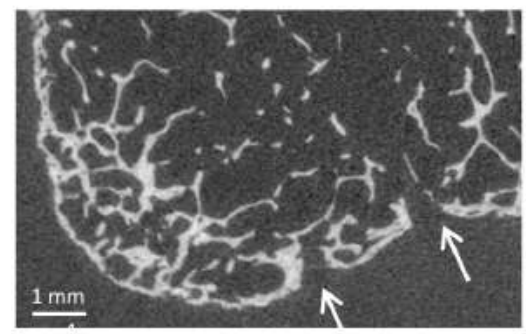

b.

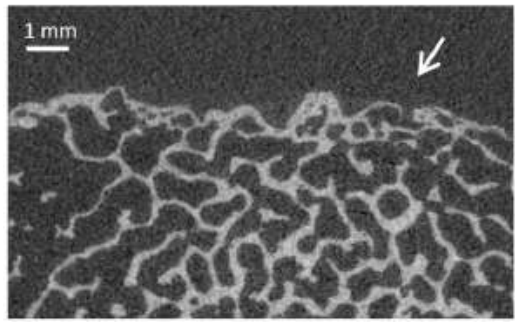

c.

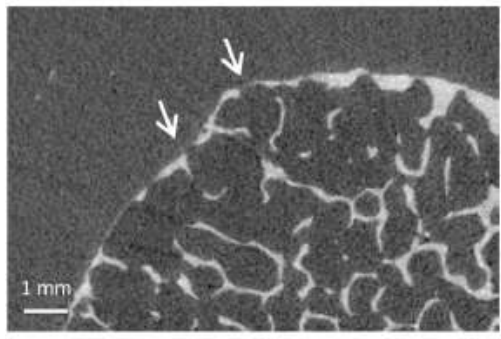

d.

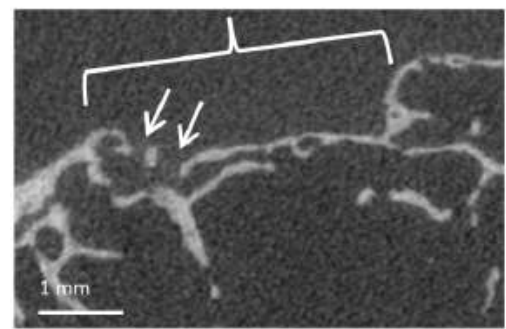

Figure S2.4. Examples of $\mu \mathrm{CT}$ images that could have attributed to the differences in scoring cortical breaks between Reader 1 and 2. (a.) Large cortical breaks (arrow) show high agreement. (b.) Small cortical breaks (arrow) show less agreement. (c.) An extremely thin cortex (arrow). (d.) One large break (in brackets) was counted by Reader 1, where Reader 2 considered this as several smaller cortical breaks (arrow). $\mu \mathrm{CT}$; microCT. 
Table S2.1. $2 \times 2$ contingency table for Reader 1 all joints (MCP and PIP)

\begin{tabular}{l|ccc}
\hline & $\mu C T$ presence break & $\mu C T$ absence break & Total \\
\hline HR-pQCT presence break & 80 & 18 & 98 \\
HR-pQCT absence break & 18 & 32 & 50 \\
Total & 98 & 50 & 148 quadrants \\
\hline
\end{tabular}

$2 \times 2$ table on the presence of a cortical break on $\mu \mathrm{CT}$ (gold standard) and HR-pQCT of Reader 1 for all joints (MCP and PIP). Reader 1 considered 4 quadrants not evaluable due to the quality of the images due to a protocol error.

Table S2.2. $2 \times 2$ contingency table for Reader 1 for MCP joints

\begin{tabular}{l|ccc}
\hline & $\mu C T$ presence break & $\mu$ CT absence break & Total \\
\hline HR-pQCT presence break & 46 & 7 & 53 \\
HR-pQCT absence break & 10 & 13 & 23 \\
Total & 56 & 20 & 76 quadrants \\
\hline
\end{tabular}

$2 \times 2$ table on the presence of a cortical break on $\mu \mathrm{CT}$ (gold standard) and HR-pQCT of Reader 1 for MCP joints. Reader 1 considered 4 quadrants not evaluable due to the quality of the images due to a protocol error.

Table S2.3. $2 \times 2$ contingency table for Reader 1 for PIP joints

\begin{tabular}{l|ccc}
\hline & $\mu C T$ presence break & $\mu$ CT absence break & Total \\
\hline HR-pQCT presence break & 34 & 11 & 45 \\
HR-pQCT absence break & 8 & 19 & 27 \\
Total & 42 & 30 & 72 quadrants \\
\hline
\end{tabular}

$2 \times 2$ table on the presence of a cortical break on $\mu \mathrm{CT}$ (gold standard) and HR-pQCT of Reader 1 for PIP joints. Reader 1 considered 4 quadrants not evaluable due to the quality of the images due to a protocol error.

Table S2.4. $2 \times 2$ contingency table for Reader 2 for all joints (MCP and PIP joints)

\begin{tabular}{l|ccc}
\hline & $\mu C T$ presence break & $\mu C T$ absence break & Total \\
\hline HR-pQCT presence break & 71 & 15 & 86 \\
HR-pQCT absence break & 32 & 34 & 66 \\
Total & 103 & 49 & 152 quadrants \\
\hline
\end{tabular}

$2 \times 2$ table on the presence of a cortical break on $\mu \mathrm{CT}$ (gold standard) and HR-pQCT of Reader 2 for all joints (MCP and PIP).

Table S2.5. $2 \times 2$ contingency table for Reader 2 for MCP joints

\begin{tabular}{l|ccc}
\hline & $\mu C T$ presence break & $\mu C T$ absence break & Total \\
\hline HR-pQCT presence break & 42 & 6 & 48 \\
HR-pQCT absence break & 22 & 10 & 32 \\
Total & 64 & 16 & 80 quadrants \\
\hline
\end{tabular}

$2 \times 2$ table on the presence of a cortical break on $\mu \mathrm{CT}$ (gold standard) and HR-pQCT of Reader 2 for MCP joints.

Table S2.6. $2 \times 2$ contingency table for Reader 2 for PIP joints

\begin{tabular}{l|ccc}
\hline & $\mu C T$ presence break & $\mu C T$ absence break & Total \\
\hline HR-pQCT presence break & 31 & 7 & 38 \\
HR-pQCT absence break & 10 & 24 & 34 \\
Total & 41 & 31 & 72 quadrants \\
\hline
\end{tabular}

$2 \times 2$ table on the presence of a cortical break on $\mu \mathrm{CT}$ (gold standard) and HR-pQCT of Reader 2 for PIP joints. 
34 Chapter 2 


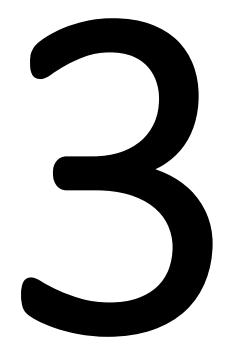

An automated algorithm for the detection of cortical interruptions on high resolution peripheral quantitative computed tomography images of finger joints

M. Peters, A. Scharmga, J. de Jong, A. van Tubergen, P. Geusens, J.J. Arts, D. Loeffen, R. Weijers, B. van Rietbergen, J. van den Bergh 
36 Chapter 3

\section{ABSTRACT}

\section{Objectives}

To introduce a fully-automated algorithm for the detection of small cortical interruptions $(\geq 0.246 \mathrm{~mm}$ in diameter) on high resolution peripheral quantitative computed tomography (HR-pQCT) images, and to investigate the additional value of manual correction of the automatically obtained contours (semi-automated procedure).

\section{Methods}

Ten metacarpophalangeal joints from seven patients with rheumatoid arthritis (RA) and three healthy subjects were imaged with HR-pQCT. The images were evaluated by an algorithm according to the fully- and semi-automated procedure for the number and surface area of interruptions per joint. Reliability between the fully- and semi-automated procedure and between two independent operators was tested using intra-class correlation coefficient (ICC) and the proportion of matching interruptions. Validity of single interruptions detected was tested by comparing it to visual scoring, as gold standard. The positive predictive value (PPV) and sensitivity were calculated.

\section{Results}

The median number of interruptions per joint was 14 (range 2 to 59) and did not significantly differ between the fully- and semi-automated procedure $(p=0.37)$. The median interruption surface area per joint was significantly higher with the fully- vs. semi-automated procedure (respectively, $8.6 \mathrm{~mm}^{2}$ vs. $5.8 \mathrm{~mm}^{2}$ and $6.1 \mathrm{~mm}^{2}, p=0.01$ ). Reliability was almost perfect between the fully- and semi-automated procedure for both the number and surface area of interruptions (ICC $\geq 0.95)$ and the proportion of matching interruptions was high $(\geq 76 \%)$. Also the inter-operator reliability was almost perfect (ICC $\geq 0.97$, proportion of matching interruptions $82 \%$ ). The PPV ranged from $27.6 \%$ to $29.9 \%$, and sensitivity from $69.7 \%$ to $76.3 \%$. Most interruptions detected with the algorithm, did show an interruption on a 2D grayscale image. However, this interruption did not meet the criteria of an interruption with visual scoring.

\section{Conclusion}

The algorithm for HR-pQCT images detects cortical interruptions, and its interruption surface area. Reliability and validity was comparable for the fully- and semi-automated procedures. However, we advise the use of the semi-automated procedure to assure quality. The algorithm is a promising tool for a sensitive and objective assessment of cortical interruptions in finger joints assessed by HR-pQCT. 
An automated algorithm for detection of cortical interruptions $\mid 37$

\section{INTRODUCTION}

Peri-articular cortical interruptions are one of the characteristic features of bone involvement in rheumatoid arthritis (RA) ${ }^{1,2}$ They may occur already at an early stage and are predictors of further radiographic progression. ${ }^{3}$ The presence, number, and size of interruptions within a joint and the number of joints affected are associated with a poor functional outcome. ${ }^{2,4,5}$

Conventional radiographs (CR) are considered the gold standard for detection of cortical interruptions in hand joints in RA. However, CR has its limitations since the sensitivity of the detection of cortical interruptions is low compared with other imaging modalities such as Computed Tomography (CT), magnetic resonance imaging (MRI) and Ultrasound (US). ${ }^{6-9}$ High resolution peripheral quantitative CT (HR-pQCT) is a low-dose imaging technique that is able to assess the three-dimensional (3D) bone structure of the peripheral skeleton at the micro-scale ( $82 \mu \mathrm{m}$ isotropic voxel size) in vivo. Studies with HR-pQCT in finger joints showed higher sensitivity for the detection of cortical interruptions compared with US, CR and MRI. ${ }^{10-13}$

Cortical interruptions, together with changes in the underlying trabecular bone architecture, such as absence of trabeculae, with or without surrounding sclerosis, are a hallmark of bone erosions. Several studies reported results on the visual inspection of the presence, number and size of cortical interruptions as part of analyzing erosions using HR-pQCT. ${ }^{10-20}$ High intra- and inter-rater reliability for the visual scoring of the number of erosions was found, with intra-class correlation coefficients (ICC) and kappa values ranging from 0.6 to $1.0 .^{10-12,18}$ However, in all studies only established erosions and thus relatively large cortical interruptions were scored (mean width $>2 \mathrm{~mm},{ }^{15,21}$ mean depth $>2 \mathrm{~mm}^{15,21}$ mean maximal dimension $>3 \mathrm{~mm}^{11}$ and mean volume $\left.>4 \mathrm{~mm}^{314,19}\right)$. Studies that systematically investigate the visual detection of smaller cortical interruptions on HR-pQCT images are currently lacking, but it is known that this is a laborious, complex and difficult process. ${ }^{22}$ Although these small interruptions might not be specific for RA, ${ }^{10}$ they might be prone to bone resorption early in the course of RA and can therefore be the first signs of erosions. ${ }^{23}$ Moreover, it has been shown that the number of these small interruptions is increased in patients with more active disease, suggesting a link to synovitis. ${ }^{10}$

Automated algorithms are an alternative to visual scoring of cortical interruptions on HRpQCT images. Recently, an algorithm has been developed that accurately measured erosion volume and erosion surface area, but still required an operator to visually identify the erosion and manually set a seeding point in the erosion. ${ }^{19} \mathrm{~A}$ fully-automated detection method of (small) cortical interruptions was not yet available.

Here, we introduce a newly developed, fully-automated algorithm for the detection of small cortical interruptions ( $\geq 0.246 \mathrm{~mm}$ in diameter) on HR-pQCT images. Because the algorithm might be susceptible for errors in the contouring, we investigated the additional value of correction of the contours by an operator (semi-automated procedure) as suggested by Burghardt et al.. ${ }^{24}$ In addition, we tested the validity of the 
$38 \mid$ Chapter 3

cortical interruptions detected by the algorithm compared to visual scoring, as gold standard.

\section{MATERIALS AND METHODS}

\section{Patients}

We selected a convenient sample of seven patients with RA (mean age 53.4 (SD 7.3) years, mean disease duration 8.2 (SD 9.8) years) and three healthy controls (HCs) (mean age 48.0 (SD 7.8) years) from the observational MOSA-Hand cohort study, including 41 patients with RA and $38 \mathrm{HCs}$ at the Maastricht University Medical Center, the Netherlands. The subjects were considered eligible when the HR-pQCT scan was of sufficient quality ( $\leq$ grade 3 , as described by Pialat et al. ${ }^{25}$ ), and the joint was not destroyed. All patients with RA fulfilled the 2010 American College of Rheumatology $(A C R) / E u r o p e a n$ League Against Rheumatism (EULAR) classification criteria for RA. ${ }^{26}$ None of the HCs suffered from hand joint complaints. Ethical approval was obtained from the ethics board of the academic hospital Maastricht, the Netherlands (NL42300.068.12 / METC 12-2-037). All participants signed informed consent.

\section{HR-pQCT scanning procedure}

MCP joints of both hands in patients with RA and the dominant hand in HCs were scanned with HR-pQCT (XtremeCT1, Scanco Medical AG, Switzerland). The scanning was performed at clinical in vivo settings, i.e. at $60 \mathrm{kVp}$ tube voltage, $900 \mu \mathrm{A}$ tube current, $100 \mathrm{~ms}$ integration time and $82 \mu \mathrm{m}$ isotropic voxel size. The reference line was placed on top of the second metacarpal head, such that the scan region covered a length of $16.56 \mathrm{~mm}$ in proximal direction and $10.50 \mathrm{~mm}$ in distal direction (total scan length $27.06 \mathrm{~mm}, 330$ slices). The total scanning time was approximately 9 minutes and the effective dose was $<9 \mu \mathrm{Sv}$. In each patient, the third MCP joint of the first scan that was available of a patient (either left or right sided) was selected for this study.

\section{Cortical interruption detection algorithm}

We developed the automated algorithm in several steps. In order to reduce the influence of noise on the images, we considered discontinuities in the cortex as cortical interruptions only if there was an opening of at least 5 voxels through the cortex and connected to both the periosteal and endosteal boundary of the cortical mask. This corresponds to a discontinuity in at least 3 consecutive slices and a width of at least 3 voxels $(=0.246 \mathrm{~mm})$. In addition, a cortical mask with a constant thickness of 4 voxels $(=0.328 \mathrm{~mm})$ was chosen because this approached the average cortical thickness in the MCP joints in this study, which was $0.39 \mathrm{~mm}$. We decided to set the thickness of the cortical mask lower, because the thickness of the cortex at the rim of the joint, where 
we expected most cortical interruptions, is in general thinner. ${ }^{14}$ The minimal intracortical interruption volume that can be detected by our algorithm is 20 voxels $\left(0.011 \mathrm{~mm}^{3}\right)$, based on an opening of at least 5 voxels * depth of 4 voxels. The 4 steps of the algorithm are explained below and displayed in Figures 3.1 and 3.2, and Supplementary Figure S3.1.

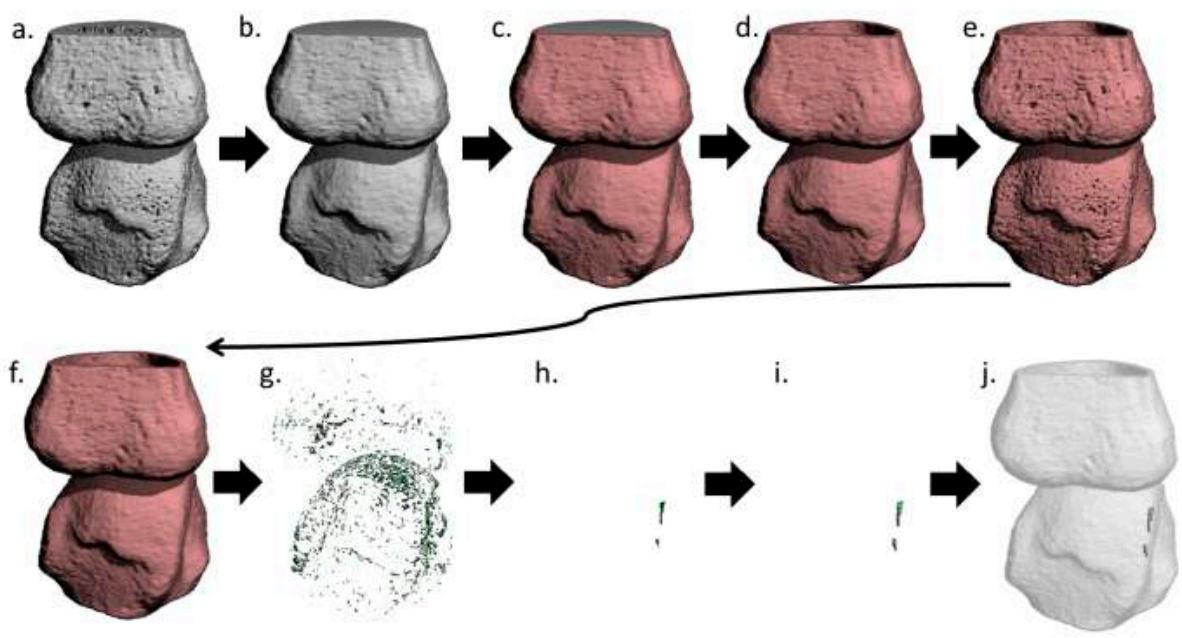

Figure 3.1. Representation of the steps executed by the algorithm. Representation of the steps executed by the algorithm applied to a 3D reconstruction of a HR-pQCT image of an MCP joint. Based on the outer margin contour of the original bone structure (a.) a solid volume is created (b.). The outer shell of this volume is segmented and depicted in red (c.) using an erosion operation. This is the cortical mask (d.), which is used to identify the cortical bone (e.). This cortical bone is dilated to fill small cavities (f.). Next, the image is inverted (g.) and only interruptions that were connected to the endosteal and periosteal boundary are selected (h.). The remaining cortical interruptions are dilated to their original volume (i.) and the results can be visually inspected by adding a transparent cortical mask (j.).

First, the outer contour of the cortex is identified. We applied a modified version of the auto-contouring algorithm developed for periosteal segmentation of the distal radius and tibia. ${ }^{24,27}$ Modifications on this algorithm were made for the structure approximation of the bone, which was performed using Gaussian filtering (sigma $=0.8$, support $=1$ voxel) with a constant threshold of 105 instead of 120 per 1000 of the maximum possible voxel value, because the cortex in finger joint is thinner and lower mineralized compared to radius and tibia in order to reduce the effect of partial volumes. In addition, and in contrast, with the originally developed algorithm, 7 instead of 10 closing steps (dilation followed by erosion in 3D) were performed, because finger joints are smaller compared to radius and tibia. This algorithm is explained in online Figure 3.1. 
40 Chapter 3

Second, a binary 3D model is created by extracting the voxels that contain mineralized bone using the standard evaluation protocol from the manufacturer for radii and tibia, which includes Laplace-Hamming filtering and thresholding. ${ }^{28}$ An example of a binary 3D model of an MCP joint is shown in Figure 3.1a.

Third, a cortical mask is generated by creating a 3D binary solid volume based on the outer margin contour (Figure 3.1b). This volume is then eroded in 3D by a distance of 4 voxels. The eroded volume is subtracted from its original volume, resulting in the outer shell of the contoured volume with a constant thickness of 4 voxels (Figure 3.1c). This shell is used as the mask (Figure 3.1d) to identify the cortical bone (Figure 3.1e).

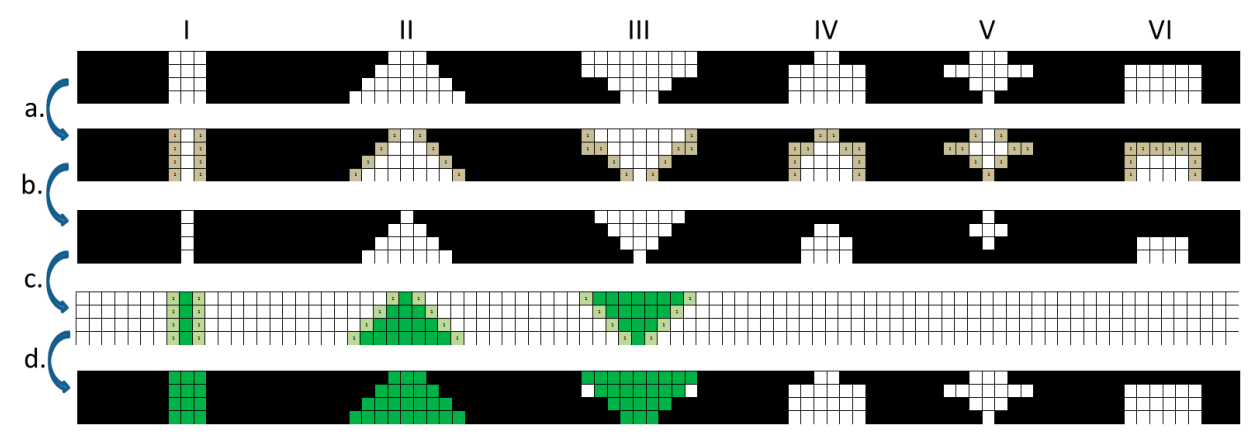

Figure 3.2. 2D examples of cortical interruptions on voxel level that are detected and are not detected by the algorithm. 2D examples of cortical interruptions on voxel level that are detected (I-III) and are not detected (IV$\mathrm{VI}$ ) by the algorithm. The following steps are made by the algorithm: The original cortex (step a, depicted in black) is dilated with 1 voxel (step b, depicted in grey). The cortex is then inverted (step c), only interruptions that are connected with the endosteal and periosteal boundary are selected and dilated to (approximately) its original size. The interruptions that are detected are subsequently displayed in the cortex (green) (d). Interruptions that are not connected to both the endosteal and periosteal boundary (IV-VI) with at least 3 pixels are not detected by the algorithm.

Fourth, the cortical bone is analyzed for discontinuities that meet the preset criteria of a cortical interruption (i.e. a connectivity of 5 voxels through the cortex and to both the periosteal and endosteal boundary of the cortical mask). First, the bone within the cortical mask is dilated in 3D by a distance of 1 voxel (Figure 3.1f). Second, the image is inverted (Figure 3.1g), and only interruptions that remain connected to both the endosteal and periosteal boundaries of the cortical mask are selected for further image processing (Figure 3.1h). These interruptions are dilated again in 3D by 1 voxel to restore the original volume (Figure 3.1i) and are considered cortical interruptions. Based on this analysis, an image can be created with a transparent cortical mask as reference (Figure 3.1j). Subsequently, the total number of cortical interruptions and the intracortical volume of each cortical interruption is determined using a function that identifies unconnected structural components and their volume. The interruption 
surface area is calculated by dividing the intra-cortical interruption volume by the thickness of the cortical mask $(=0.328 \mathrm{~mm})$. Figure 3.2 shows $2 \mathrm{D}$ examples of cortical interruptions on voxel level, which are detected and not detected by the algorithm. Only interruptions on at least 3 consecutive slices and a width of at least 3 voxels through the cortex are detected. The algorithm is enclosed as Supplementary File (File S3.1).

\section{Correction of the contours}

All HR-pQCT images were also evaluated by the semi-automated procedure in which the contour generated in step 1 of the fully-automated procedure is visually inspected and, if necessary, corrected by an operator as recommended by Burghardt et al.. ${ }^{24}$ To test the inter-operator reliability, this correction was performed by two operators independently.

\section{Visual scoring}

All HR-pQCT images were scored visually on cortical interruptions for comparison with the algorithm. A cortical interruption was defined as a clear interruption of the cortex, seen on two consecutive slices on two orthogonal planes (on transverse, and on sagittal or coronal plane). ${ }^{29,30}$

\section{Statistical analysis}

In this study, the results of the fully-automated procedure (AUTO), the two operators (OP1 and OP2) of the semi-automated procedure, and visual scoring were compared. Descriptives of the number of interruptions and the interruption surface area were calculated. Friedman test was used for comparison across the operators and visual scoring. Post-hoc analyses were performed using Wilcoxon signed-rank test between the operators, and between the operators and visual scoring.

Reliability between AUTO and the semi-automated procedure, and between OP1 and OP2 for the number of interruptions and interruption surface area on the joint level was estimated by ICCs with a two-way random model and absolute agreement. In addition, the reliability on the joint level between the algorithm (AUTO, OP1 and OP2) and visual scoring for the number of interruptions was obtained using ICCs.

Reliability was rated according to Landis et al.: $<0.00$ poor, $0.00-0.20$ slight, $0.21-0.40$ fair, 0.41-0.60 moderate, 0.61-0.80 substantial, 0.81-1.00 almost perfect. ${ }^{31}$ In addition, reliability was evaluated by the proportion of matching interruptions across the operators. Interruptions were counted as matching interruptions if they overlapped with at least 20 voxels $\left(0.011 \mathrm{~mm}^{3}\right)$, because this is the smallest intra-cortical interruption volume that could be detected with this algorithm. The proportion of matching interruptions was calculated for the presence (yes/no) of a cortical interruption on exactly the same location according to equation 1 . Moreover, the validity of the algorithm to detect single interruptions on exactly the same location was evaluated by calculation of the positive predictive value (PPV, equation 2), and sensitivity (equation 3 ) 
using the visual scoring as gold standard. Statistical analysis was performed using IBM SPSS Statistics for Windows, Version 20.0 (IBM Corp., Armonk, NY).

Proportion of matching interruptions $=\frac{\mathrm{nr} \text {. of matching interruptions } \mathrm{OP} 12}{\mathrm{nr} \text {. of interruptions } \mathrm{OP} 1_{\mathrm{O} 1}+\mathrm{nr} \text {. of interruptions } \mathrm{OP} 2_{2}-\mathrm{nr} \text {. of matching interruptions } \mathrm{OP} 12} * 100 \%$

Nr. of interruptions $\mathrm{Op}_{1}$ :

Nr. of interruptions $\mathrm{OP}_{2}$ :

$\mathrm{Nr}$. of matching interruptions $\mathrm{OP}_{12}$ :

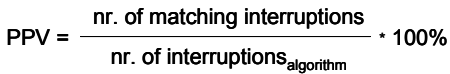

PPV:

Nr. of interruptions algorithm:

$\mathrm{Nr}$. of matching interruptions:
Number of interruptions detected by $1^{\text {st }}$ operator Number of interruptions detected by $2^{\text {nd }}$ operator Number of matching interruptions between $1^{\text {st }}$ and $2^{\text {nd }}$ operator

Positive predictive value

Number of interruptions detected by the algorithm Number of matching interruptions between the algorithm and visual scoring

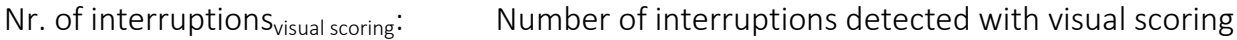
Nr. of matching interruptions: Number of matching interruptions between the algorithm and visual scoring

\section{RESULTS}

\section{Visual impression}

\section{Output algorithm}

Both the fully- and semi-automated procedures detected multiple cortical interruptions in $\mathrm{HCs}$ and patients with RA (Figure 3.3). Figure 3.3 shows a typical example of 3D reconstructions of an MCP joint of an $\mathrm{HC}(\mathrm{I})$, a patient with early RA $(<2$ years since diagnosis) (II), and a patient with late RA (>10 years since diagnosis) (III), and the detected interruptions of the fully-automated procedure. Multiple cortical interruptions (depicted in green) can be seen in the 3D images and most interruptions are located at the rim of the joint (Figure 3.3a). Figure 3.3b shows corresponding 2D grayscale images of examples of interruptions that were detected by the fully-automated as well as the semi-automated procedure. 
a.
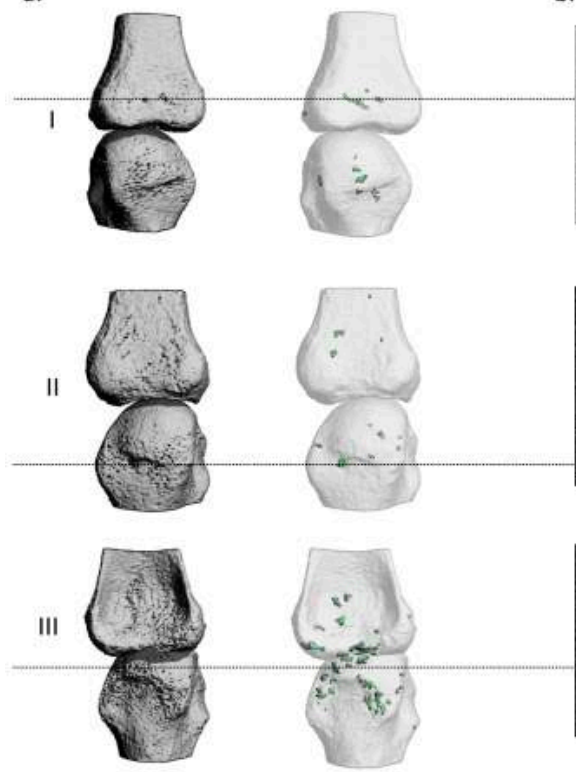

b.
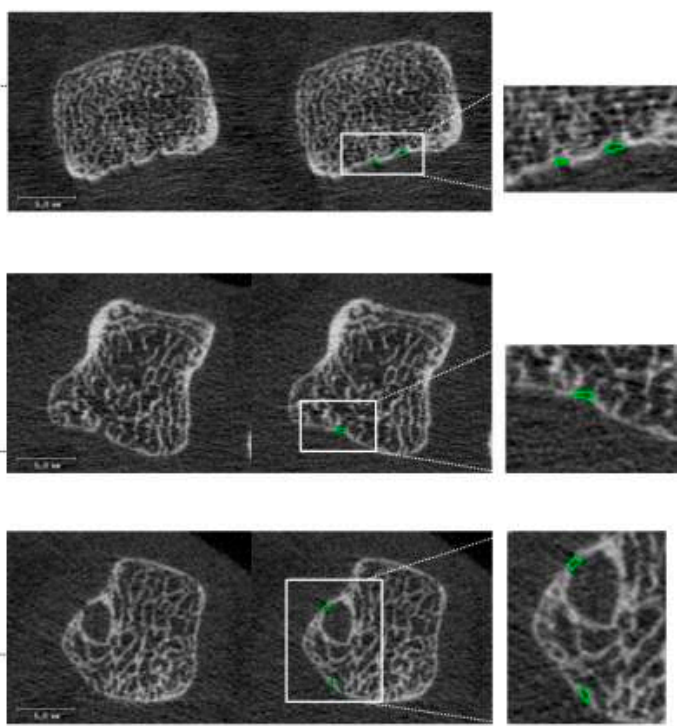

Figure 3.3. Examples of the $2 \mathrm{D}$ and $3 \mathrm{D}$ outputs of the algorithm. Typical examples of a 3D reconstruction of an MCP joint for a $\mathrm{HC}(\mathrm{I})$, a patient with early RA (<2 years since diagnosis) (II), and a patient with late RA ( $>10$ years since diagnosis) (III), with the corresponding 3D outputs of the algorithm. The cortical region is indicated in transparent white. Cortical interruptions of $\geq 0.246 \mathrm{~mm}$ that are detected by the algorithm are shown in green. b) Corresponding 2D grayscale images with in green cortical interruptions detected by the algorithm.

\section{Correction of the contours}

Figure 3.4 shows examples of contours obtained with the fully-automated procedure that were manually corrected by the operators. In two cases, large cortical interruptions were corrected by both operators. For small cortical interruptions (diameter $<1 \mathrm{~mm}$ ) no correction was necessary by the operators. Figure 3.4a-l shows an example of a large cortical interruption (diameter $>1.0 \mathrm{~mm}$, white arrow). The contour obtained with the fully-automated procedure did not follow the outer margin of the original structure at the location of the cortical interruption (Figure 3.4a-II). Both operators corrected the contour (Figure 3.4a-III, white arrow) to more precisely detect the size of the large cortical interruption (Figure 3.4a-IV) 
44 $\mid$ Chapter 3
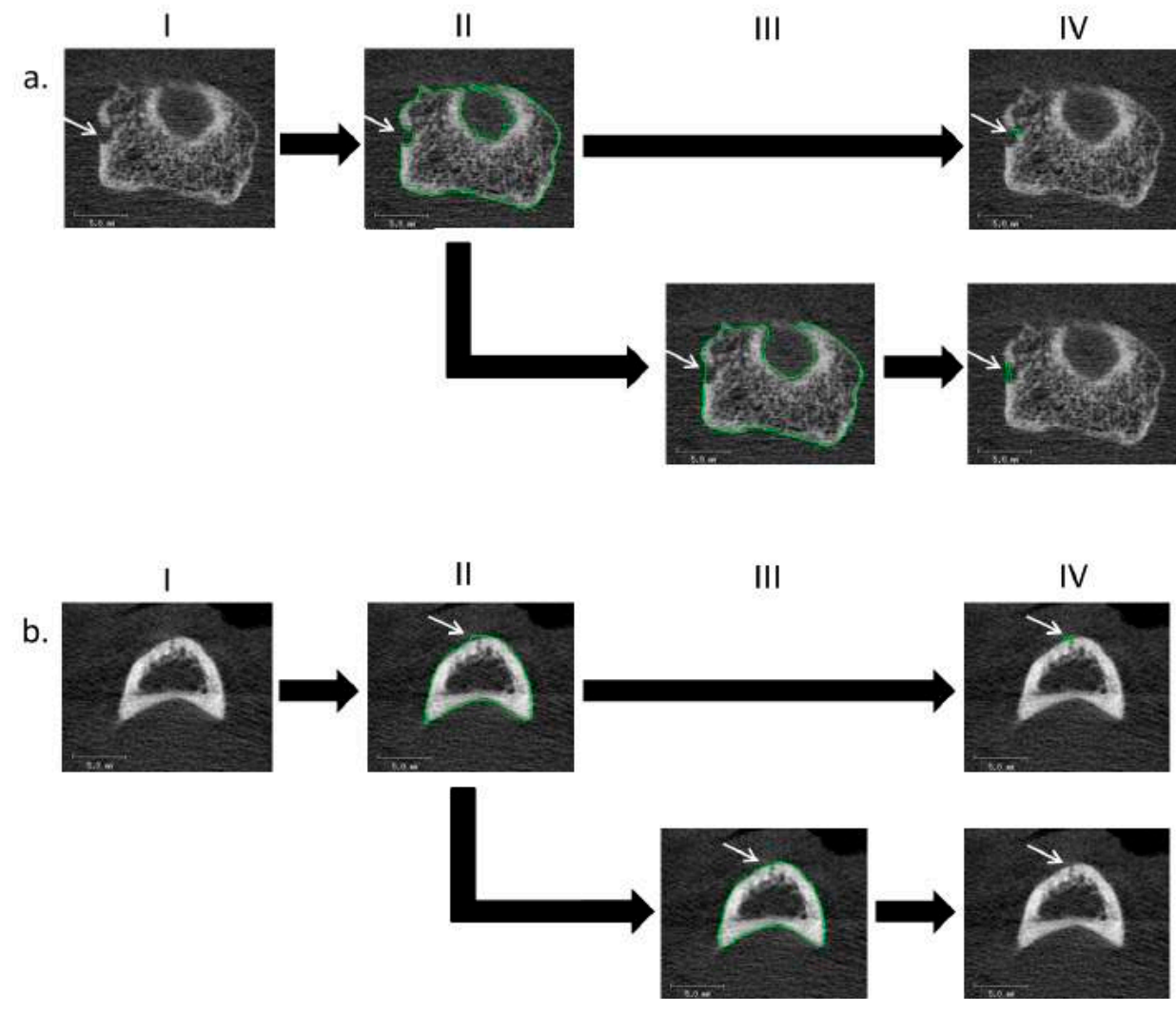

Figure 3.4. Examples of contours that were manually corrected. Typical examples of 2D grayscale images in which the contour is manually corrected by the operators. In a) a large cortical interruption is shown (a. I, arrow). The automatically obtained contour does not follow the outer margin of the original structure at the cortical interruption (a. II). The operators therefore corrected the contour (a. III) to accurately detect the large cortical interruption (IV). In b) a small motion artifact is shown (I). Due to this motion artifact, the automatically obtained contour was not tight around its original structure (II). The operators corrected this (III) and therefore no interruption was detected (IV).

Figure 3.4b-I shows an example of a small motion artifact (white arrow). Due to this motion artifact, the contour obtained with the fully-automated procedure was not tight around the original structure (Figure 3.4b-II), which led to a false detection of an interruption (Figure 3.4b-IV). Both operators corrected the contour (Figure 3.4b-IV).

\section{Quantitative comparison}

Table 3.1 shows the number of interruptions and interruption surface area detected by the algorithm (AUTO, OP1 and OP2), and visual scoring. The median number of interruptions was 14 and ranged from 2 to 59 interruptions per joint for the algorithm when including all operators. The median number of interruptions with visual scoring 
was 5 and ranged from 3 to 22 interruptions per joint. Statistical significant differences were found across the algorithm and visual scoring for the number of interruptions $(p<0.01)$. The post-hoc analysis showed significant differences between the algorithm (AUTO, OP1 and OP2) versus visual scoring (all, $p \leq 0.01$ ).

Table 3.1. The number of cortical interruptions and interruption surface area per joint detected by the algorithm and with visual scoring

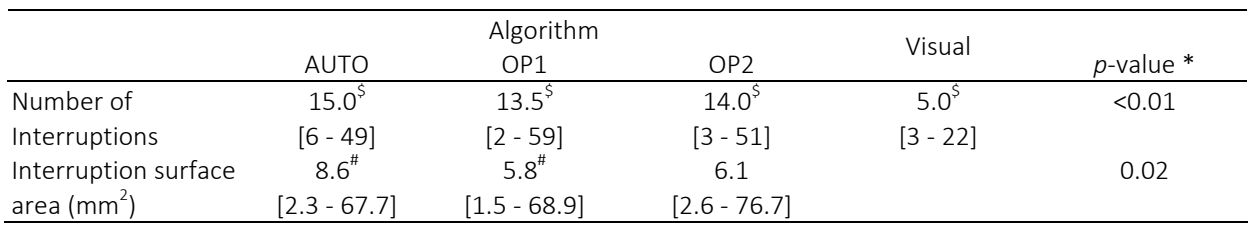

Values are displayed as: median [min-max], AUTO =fully-automated procedure. OP1 =semi-automated procedure manual correction by operator 1 . OP2 =semi-automated procedure manual correction by operator 2. * $p$-values obtained from Friedman test. $\$ p \leq 0.01$ obtained from post-hoc Wilcoxon signed-rank test (algorithm (AUTO, OP1 and OP2) vs. visual). \# $p<0.05$ obtained from post-hoc Wilcoxon signed-rank test (AUTO vs. OP1)

The median of the interruption surface area per joint was $7.0 \mathrm{~mm}^{2}$ and ranged from $1.5 \mathrm{~mm}^{2}$ to $76.7 \mathrm{~mm}^{2}$ when including all operators. The average surface area of a single interruption was $<1.0 \mathrm{~mm}^{2}$. The interruption surface area per joint was significantly lower with the semi-automated vs. the fully-automated procedure (Table 3.1). The posthoc analysis showed a significant difference between AUTO and OP1 ( $p=0.02)$, but no significant difference between AUTO and OP2 ( $p=0.07)$, and OP1 and OP2 ( $p=0.29)$.

The reliability between AUTO and both OP1 and OP2 was almost perfect for both the total number of interruptions and the interruption surface area (ICC $\geq 0.95, p<0.01)$. The majority of interruptions were detected on exactly the same location $(\geq 76.6 \%)$ (Table 3.2). The inter-operator reliability was also almost perfect (ICC $\geq 0.97, p<0.01$ ), as was the proportion of matching interruptions (82.0\%).

Table 3.2. Reliability of the algorithm.

\begin{tabular}{lccc}
\hline & AUTO vs. OP1 & AUTO vs. OP2 & Inter-operator \\
\hline Number of Interruptions & ICC 0.96 & ICC 0.95 & ICC 0.97 \\
& $(95 \% \mathrm{Cl} 0.87-0.99)$ & $(95 \% \mathrm{Cl} 0.83-0.99)$ & $(95 \% \mathrm{Cl} 0.90-0.99)$ \\
Interruption Surface area & ICC 0.98 & ICC 0.96 & ICC 0.98 \\
& $(95 \% \mathrm{Cl} 0.77-1.00)$ & $(95 \% \mathrm{Cl} 0.85-0.99)$ & $(95 \% \mathrm{Cl} 0.92-1.00)$ \\
Proportion of matching Interruptions & $81.1 \%$ & $76.6 \%$ & $82.0 \%$ \\
\hline
\end{tabular}

Reliability of the fully-automated vs. semi-automated (AUTO vs. OP1 and AUTO vs. OP2) and semi-automated inter-operator (OP1 vs. OP2) of the algorithm is shown. ICCS are calculated on the total number of cortical interruptions and interruption surface area in all joints. Proportion of matching interruptions is calculated on the presence of a cortical interruption (yes/no) on exactly the same location. ICC =intra-class correlation coefficient, $95 \% \mathrm{Cl}=95 \%$ confidence interval 
46 $\mid$ Chapter 3

\section{Comparison to visual scoring}

The reliability between the algorithm (AUTO, OP1 and OP2), and visual scoring was fair to moderate for the number of interruptions (ICC ranging from 0.38 to 0.45 ). The PPV of interruptions detected by the algorithm compared to visual scoring was fair (PPV ranging from $27.6 \%$ to $29.9 \%$ ). The sensitivity of the algorithm compared to visual scoring was substantial (sensitivity ranging from $69.7 \%$ to $76.3 \%$ ). Examples of interruptions detected visually and with the algorithm are shown in Figure 3.5. An example of an interruption detected visually as well as with the algorithm is shown (Figure 3.5-I). Figure 3.5-II shows interruptions detected with the algorithm which did not meet with the criteria of visual scoring. Figure 3.5-III shows an interruption that was detected visually, but missed by the algorithm.

a.
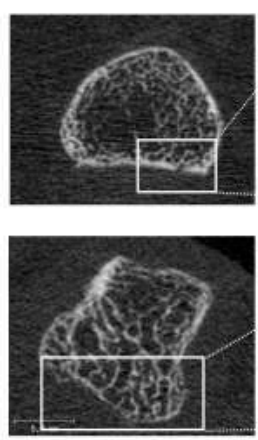

III

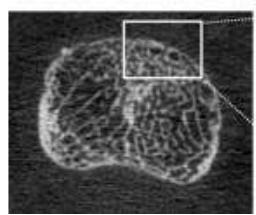

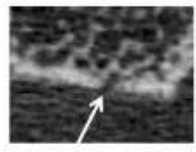
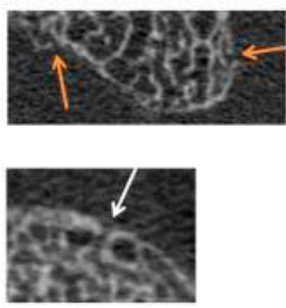

b.
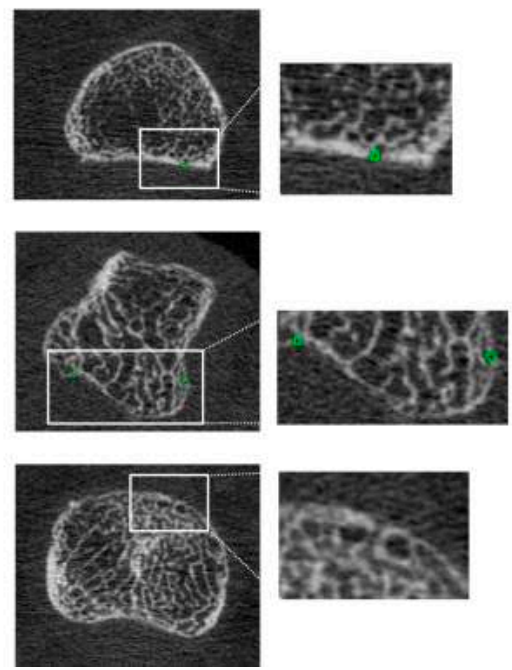

Figure 3.5. 2D examples of interruptions detected visually, and with the algorithm. Typical examples of a 2D grayscale images of interruptions detected visually (a), and with the algorithm (b). (I.) Shows an interruption detected visually (white arrow) as well as with the algorithm (green circle). (II.) Shows interuptions detected by the algorithm which were not detected visually (orange arrows). Small cortical interruptions could be observed, but not meeting the criteria of a cortical interruption in the visual scoring (II.). (III.) Shows a small interruption that was detected visually (white arrow), but not with the algorithm.

\section{DISCUSSION}

In this study, we introduced a fully-automated algorithm to detect small cortical interruptions in HR-pQCT images and tested the additional value of correction by an 
operator. In addition, we tested the validity of interruptions detected by the algorithm by comparing it to visual scoring, as gold standard. The interruption surface area detected with the fully-automated procedure was significantly higher compared with the semi-automated procedure, but the absolute number of interruptions did not significantly differ. Reliability was almost perfect between both procedures (ICC $\geq 0.95$ ) meaning that the manual correction of the contours was of little additional value. In addition, the proportion of matching interruptions on exactly the same location was substantial to almost perfect across the operators ( $\geq 76.6 \%)$. Lastly, the validity of a single interruption was similar for both procedures. We found that most interruptions detected by the algorithm were not identified as a cortical interruption with the visual scoring (70-72\%). However, in most cases an interruption was present, but did not met the preset criteria (Figure 3.5-II). In addition, most interruptions detected visually were detected by the algorithm (70-76\%). However, it remains important that the images are rated on motion artifacts before applying the fully-automated algorithm. Only a scan quality with grade $\leq 3$ according to Pialat et al.. ${ }^{25}$ should be included in the analysis. Even small motion artifacts may lead to false positive detection of cortical interruptions as exemplified in this study (Figure 3.4b). Compared to visual scoring of the number of cortical interruptions, ${ }^{10-19}$ our approach has the advantage of providing an automated and objective measure of evaluation of cortical interruptions and its interruption surface area, including small interruptions.

The number of cortical interruptions per joint detected in our study (median 14, ranging from 2 to 59) is substantially higher than observed in other studies using HR-pQCT, ranging from 0.5 to 2.9 interruptions per MCP joint. ${ }^{10,11,15,16,18,19,21}$ This can mainly be explained by the fact that our algorithm detects smaller cortical interruptions (mean interruption surface area $<1 \mathrm{~mm}^{2}$, equivalent to a width of $<1.13 \mathrm{~mm}$ when assuming a circle) compared with a visual scoring of the number of interruptions (mean width $>2 \mathrm{~mm}^{15,21}$ ). Furthermore, in these studies the number of interruptions was scored on 2D grayscale images whereas our automated algorithm detects interruptions on 3D binary images, which has the advantage of having an objective manner to distinguish between bone and non-bone. The use of binary images has been validated earlier in human tibia and radius. ${ }^{32,33}$ A potential disadvantage of binary images is the partial volume effect, which mainly affects regions where the cortical bone is thin, i.e. close to the image spatial resolution, leading to the misperception that an interruption is present in the binary image. We showed examples of interruptions that were detected with the algorithm displayed in 2D grayscale images (Figures 3.3 and 3.5). In most cases, a clear interruption in the cortex can be seen, whereas in some cases an interruption was detected which could not be seen on the $2 \mathrm{D}$ grayscale images, as a result of the partial volume effect (Figure 3.3 and 3.5). This potential error might be resolved when using the second generation HR-pQCT (HR-pQCT 2). The HR-pQCT 2 has a higher spatial resolution compared to the HR-pQCT (95 $\mu \mathrm{m}$ versus $130 \mu \mathrm{m}$, respectively). Thus, the HR-pQCT 2 might be able to detect these thin structures, which may reduce the number of interruptions detected as a result of the partial volume effect. 
However, with the HR-pQCT 2, the cortical mask thickness should be increased to 5 voxels $(=0.305 \mathrm{~mm})$, to obtain a similar cortical mask thickness as for the first generation HR-pQCT. In addition, the minimum diameter of interruptions is smaller on the HR-pQCT 2 due to the smaller voxel size ( $\geq 0.183 \mathrm{~mm}$ versus $\geq 0.246 \mathrm{~mm}$ ). Hence, the HR-pQCT 2 is able to detect even smaller interruptions, which might lead to a higher number of interruptions detected.

The fully-automated differed from the semi-automated procedure in the detection of large cortical interruptions (diameter $>1 \mathrm{~mm}$ ). In the first step of the fully-automated algorithm, where the outer contour of the bone is identified, the original outer contour of the finger joint may not be restored in the case of large interruptions. In the example of Figure 3.4a, both operators manually corrected this. For small cortical interruptions (diameter $<1 \mathrm{~mm}$ ) no correction was necessary. The role for HR-pQCT will mainly be for the detection of small cortical interruptions, as larger interruptions are already visible on other imaging techniques. Although not specific for RA, ${ }^{10}$ the small interruptions might be prone to bone resorption early in the course of RA. ${ }^{23}$ Clinical follow-up studies in patients with RA are needed to investigate whether these small interruptions may become established erosions.

Some limitations of the study should be mentioned. First, we included a small number of subjects, however, a large number of cortical interruptions (199 in total) was detected in these joints, which provided us reliable estimates for the proportion of matching interruptions. Second, the cortical mask was set to a constant thickness because this would avoid any inaccuracies in the detection of the endosteal cortical bone contour. A disadvantage of our approach is that in relatively thick cortical regions, that are located more distal from the joint, cortical interruptions can be detected which are not connected to the endosteal cortical bone surface, as was exemplified in Figure 3.4b. In addition, the interruption surface area may be overestimated in thin cortical regions, because a small part of the trabecular region is considered as part of the interruption.

As RA is characterized by the development of cortical interruptions in hand joints, we expect that this fully-automated algorithm can help in the assessment of small cortical interruptions in finger joints by HR-pQCT. The algorithm needs to be further validated in clinical studies in order to obtain its potential value.

In conclusion, the algorithm presented in this study allows the detection of cortical interruptions, and its interruption surface area. Reliability and validity was comparable for the fully- and semi-automated procedures. However, we advise the use of the semiautomated procedure to assure quality. We expect that this algorithm is a promising tool for a sensitive and objective assessment of cortical interruptions in finger joints by HR-pQCT 


\section{REFERENCES}

1. Schett G, Gravallese E. Bone erosion in rheumatoid arthritis: mechanisms, diagnosis and treatment. Nat Rev Rheumatol. 2012;8(11):656-64.

2. Geusens P, van den Bergh J. Bone erosions in rheumatoid arthritis. Rheumatology (Oxford). 2014;53(1):4-5.

3. Hulsmans HM, Jacobs JW, van der Heijde DM, van Albada-Kuipers GA, Schenk Y, Bijlsma JW. The course of radiologic damage during the first six years of rheumatoid arthritis. Arthritis Rheum. 2000;43(9): 1927-40.

4. van der Heijde DM, van Riel PL, van Leeuwen MA, van 't Hof MA, van Rijswijk MH, van de Putte LB. Prognostic factors for radiographic damage and physical disability in early rheumatoid arthritis. A prospective follow-up study of 147 patients. Br J Rheumatol. 1992;31(8):519-25.

5. Lillegraven S, van der Heijde D, Uhlig T, Kvien TK, Haavardsholm EA. What is the clinical relevance of erosions and joint space narrowing in RA? Nat Rev Rheumatol. 2012;8(2):117-20.

6. Ejbjerg B, Narvestad E, Rostrup E, Szkudlarek M, Jacobsen S, Thomsen HS, et al. Magnetic resonance imaging of wrist and finger joints in healthy subjects occasionally shows changes resembling erosions and synovitis as seen in rheumatoid arthritis. Arthritis Rheum. 2004;50(4):1097-106.

7. Baillet A, Gaujoux-Viala C, Mouterde G, Pham T, Tebib J, Saraux A, et al. Comparison of the efficacy of sonography, magnetic resonance imaging and conventional radiography for the detection of bone erosions in rheumatoid arthritis patients: a systematic review and meta-analysis. Rheumatology (Oxford). 2011;50(6):1137-47.

8. Geusens P, Chapurlat R, Schett G, Ghasem-Zadeh A, Seeman E, de Jong J, et al. High-resolution in vivo imaging of bone and joints: a window to microarchitecture. Nat Rev Rheumatol. 2014;10(5):304-13.

9. Dohn UM, Ejbjerg BJ, Hasselquist M, Narvestad E, Moller J, Thomsen HS, et al. Detection of bone erosions in rheumatoid arthritis wrist joints with magnetic resonance imaging, computed tomography and radiography. Arthritis Res Ther. 2008;10(1):R25.

10. Stach CM, Bauerle M, Englbrecht M, Kronke G, Engelke K, Manger B, et al. Periarticular bone structure in rheumatoid arthritis patients and healthy individuals assessed by high-resolution computed tomography. Arthritis Rheum. 2010;62(2):330-9.

11. Srikhum W, Virayavanich W, Burghardt AJ, Yu A, Link TM, Imboden JB, et al. Quantitative and semiquantitative bone erosion assessment on high-resolution peripheral quantitative computed tomography in rheumatoid arthritis. J Rheumatol. 2013;40(4):408-16.

12. Albrecht A, Finzel S, Englbrecht M, Rech J, Hueber A, Schlechtweg $P$, et al. The structural basis of MRI bone erosions: an assessment by microCT. Ann Rheum Dis. 2013;72(8):1351-7.

13. Finzel S, Ohrndorf S, Englbrecht M, Stach C, Messerschmidt J, Schett G, et al. A detailed comparative study of high-resolution ultrasound and micro-computed tomography for detection of arthritic bone erosions. Arthritis Rheum. 2011;63(5):1231-6.

14. Fouque-Aubert A, Boutroy S, Marotte H, Vilayphiou N, Bacchetta J, Miossec P, et al. Assessment of hand bone loss in rheumatoid arthritis by high-resolution peripheral quantitative CT. Ann Rheum Dis. 2010;69(9):1671-6.

15. Finzel S, Rech J, Schmidt S, Engelke K, Englbrecht M, Stach C, et al. Repair of bone erosions in rheumatoid arthritis treated with tumour necrosis factor inhibitors is based on bone apposition at the base of the erosion. Ann Rheum Dis. 2011;70(9):1587-93.

16. Finzel S, Englbrecht M, Engelke K, Stach C, Schett G. A comparative study of periarticular bone lesions in rheumatoid arthritis and psoriatic arthritis. Ann Rheum Dis. 2011;70(1):122-7.

17. Kocijan R, Finzel S, Englbrecht M, Engelke K, Rech J, Schett G. Decreased quantity and quality of the periarticular and nonperiarticular bone in patients with rheumatoid arthritis: a cross-sectional HR-pQCT study. J Bone Miner Res. 2014;29(4):1005-14.

18. Aschenberg S, Finzel S, Schmidt S, Kraus S, Engelke K, Englbrecht M, et al. Catabolic and anabolic periarticular bone changes in patients with rheumatoid arthritis: a computed tomography study on the role of age, disease duration and bone markers. Arthritis Res Ther. 2013;15(3):R62. 
50 Chapter 3

19. Topfer D, Finzel S, Museyko O, Schett G, Engelke K. Segmentation and quantification of bone erosions in high-resolution peripheral quantitative computed tomography datasets of the metacarpophalangeal joints of patients with rheumatoid arthritis. Rheumatology. 2014;53(1):65-71.

20. Barnabe C, Szabo E, Martin L, Boyd SK, Barr SG. Quantification of small joint space width, periarticular bone microstructure and erosions using high-resolution peripheral quantitative computed tomography in rheumatoid arthritis. Clin Exp Rheumatol. 2013;31(2):243-50.

21. Finzel S, Rech J, Schmidt S, Engelke K, Englbrecht M, Schett G. Interleukin-6 receptor blockade induces limited repair of bone erosions in rheumatoid arthritis: a micro CT study. Ann Rheum Dis. 2013;72(3): 396-400.

22. Zebaze R, Seeman E. Cortical bone: a challenging geography. J Bone Miner Res. 2015;30(1):24-9.

23. Schett G, Gravallese E. Bone erosion in rheumatoid arthritis: mechanisms, diagnosis and treatment. Nat Rev Rheumatol. 2012;8(11):656-64.

24. Burghardt AJ, Buie HR, Laib A, Majumdar S, Boyd SK. Reproducibility of direct quantitative measures of cortical bone microarchitecture of the distal radius and tibia by HR-pQCT. Bone. 2010;47(3):519-28.

25. Pialat JB, Burghardt AJ, Sode M, Link TM, Majumdar S. Visual grading of motion induced image degradation in high resolution peripheral computed tomography: impact of image quality on measures of bone density and micro-architecture. Bone. 2012;50(1):111-8.

26. Aletaha D, Neogi T, Silman AJ, Funovits J, Felson DT, Bingham CO, 3rd, et al. 2010 Rheumatoid arthritis classification criteria: an American College of Rheumatology/European League Against Rheumatism collaborative initiative. Arthritis Rheum. 2010;62(9):2569-81.

27. Buie HR, Campbell GM, Klinck RJ, MacNeil JA, Boyd SK. Automatic segmentation of cortical and trabecular compartments based on a dual threshold technique for in vivo micro-CT bone analysis. Bone. 2007;41(4):505-15.

28. Laib A, Hauselmann HJ, Ruegsegger P. In vivo high resolution 3D-QCT of the human forearm. Technol Health Care. 1998;6(5-6):329-37.

29. Scharmga A, Peters M, van Tubergen A, van den Bergh J, de Jong J, Loeffen D, et al. Visual detection of cortical breaks in hand joints: reliability and validity of high-resolution peripheral quantitative CT compared to microCT. BMC Musculoskelet Disord. 2016;17(1):271.

30. Barnabe C, Toepfer D, Marotte H, Hauge EM, Scharmga A, Kocijan R, et al. Definition for Rheumatoid Arthritis Erosions Imaged with High Resolution Peripheral Quantitative Computed Tomography and Interreader Reliability for Detection and Measurement. J Rheumatol. 2016;43(10):1935-40.

31. Landis JR, Koch GG. The measurement of observer agreement for categorical data. Biometrics. 1977; 33(1):159-74.

32. MacNeil JA, Boyd SK. Accuracy of high-resolution peripheral quantitative computed tomography for measurement of bone quality. Med Eng Phys. 2007;29(10):1096-105.

33. Liu XS, Zhang XH, Sekhon KK, Adams MF, McMahon DJ, Bilezikian JP, et al. High-resolution peripheral quantitative computed tomography can assess microstructural and mechanical properties of human distal tibial bone. J Bone Miner Res. 2010;25(4):746-56. 
a.

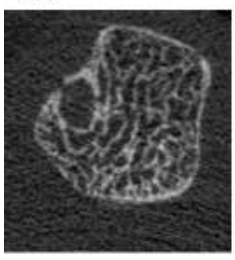

b.
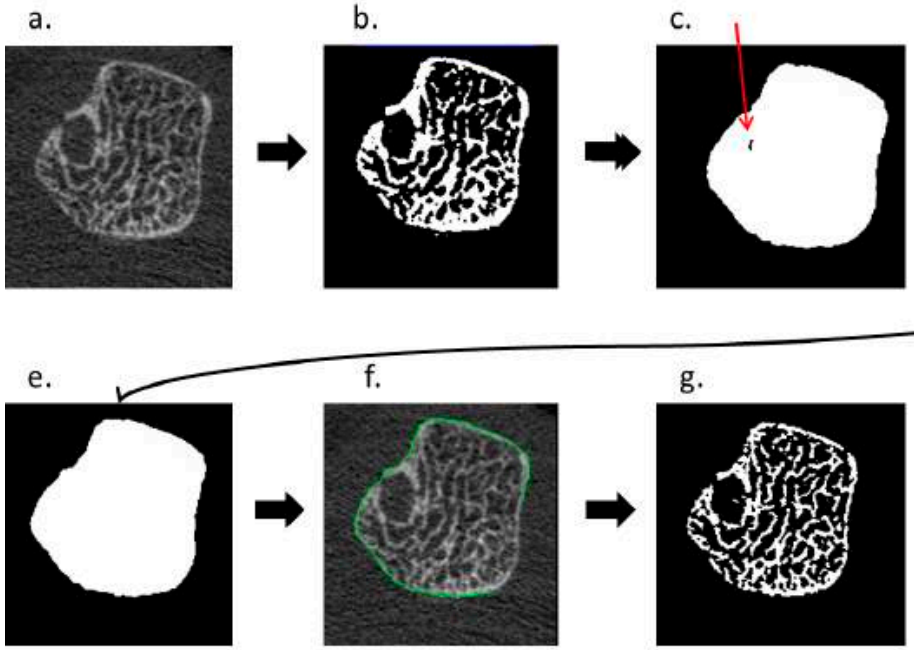

f.

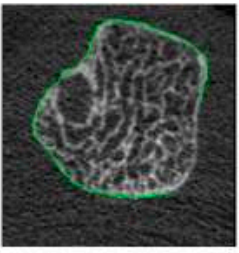

g.

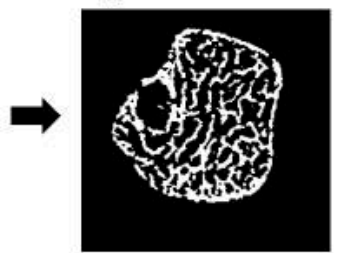

Figure S3.1. An illustration of the auto-contouring script. An illustration of the auto-contouring script of a 2D grayscale image with a cortical interruption. The grayscale image (a) is thresholded for a first structure approximation using Gaussian filtering (sigma=0.8, support=1 voxel) and a constant threshold of 105 per 1000 of maximum possible voxel value (b). This structure is dilated by 7 voxels (c). After deleting (by ranking) the black voxels inside the dilated structure (red arrow, d), the volume was eroded back to its original size (e). The contour that is obtained is displayed in green in the original grayscale image (f), and used for segmentation of the bone using the standard evaluation protocol with a constant threshold using Laplace-Hamming filtering (g). 



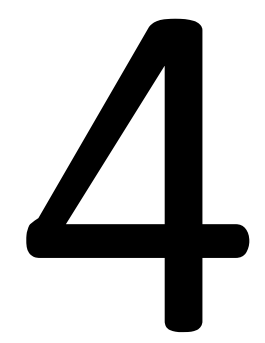

The reliability of a semi-automated algorithm for detection of cortical interruptions in finger joints on high resolution CT compared to microCT

M. Peters, A. Scharmga, A. van Tubergen, J. Arts, D. Loeffen, R. Weijers, B. van Rietbergen, P. Geusens, J.P. van den Bergh Calcified tissue international 2017 Aug;101(2):132-40. 


\section{ABSTRACT}

\section{Background}

We developed a semi-automated algorithm for the detection of cortical interruptions in finger joints using high-resolution peripheral quantitative computed tomography (HR-pQCT). Here, we tested its reliability compared to microCT $(\mu \mathrm{CT})$ as gold standard.

\section{Methods}

Nineteen joints of 10 female anatomic index fingers were imaged by HR-pQCT and $\mu C T$ (82 and $18 \mu \mathrm{m}$ isotropic voxel sizes, respectively). The algorithm was applied for detection of cortical interruptions of different minimum diameters (range $>0.16 \mathrm{~mm}$ to $>0.50 \mathrm{~mm}$ ). Reliability was tested at the joint level with intra-class correlation coefficient (ICC) for the number of interruptions and interruption surface, and at the level of a single interruption for matching between HR-pQCT and $\mu \mathrm{CT}$ with a fixed interruption diameter $(>0.10 \mathrm{~mm})$ on $\mu \mathrm{CT}$. The positive predictive value $\left(\mathrm{PPV}_{0.10 \mathrm{~mm}}\right)$ and sensitivity $0.10 \mathrm{~mm}$ were evaluated.

\section{Results}

The mean number of interruptions per joint depended on the diameter cut-off and ranged from 3.4 to 53.5 on HR-pQCT and from 1.8 to 45.1 on $\mu \mathrm{CT}$ for interruptions $>0.50 \mathrm{~mm}$ to $>0.16 \mathrm{~mm}$, respectively. Reliability at the joint level was almost perfect (ICC $\geq 0.81$ ) for both the number and surface of interruptions $>0.16 \mathrm{~mm}$ and $>0.33 \mathrm{~mm}$. As expected, the $P P V_{0.10 m m}$ increased with increasing interruption diameter from $84.9 \%$ to $100 \%$, for interruptions $>0.16 \mathrm{~mm}$ and $>0.50 \mathrm{~mm}$, respectively. However, the sensitivity ${ }_{0.10 \mathrm{~mm}}$ decreased with increasing interruption diameter from $62.4 \%$ to $4.7 \%$.

\section{Conclusion}

This semi-automated algorithm for HR-pQCT in finger joints performed best for the detection of cortical interruptions with a minimum diameter of $>0.16 \mathrm{~mm}$ or $>0.33 \mathrm{~mm}$, showing almost perfect reliability at the joint level and interruptions matched with those on $\mu \mathrm{CT}$. 


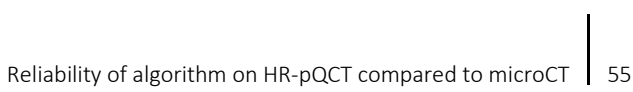

\section{INTRODUCTION}

Peri-articular cortical interruptions are one of the characteristic features of bone involvement in rheumatoid arthritis (RA) and predictors of further radiographic progression. ${ }^{1,2}$ Conventional radiographs (CR) are considered the gold standard for detection of cortical interruptions (i.e. erosions) in the hand joints in RA in daily practice. However, CRs have limited sensitivity to visualize joint damage compared to Computed Tomography (CT), MRI and Ultrasound (US). ${ }^{3-6}$

High-resolution peripheral quantitative CT (HR-pQCT) is a non-invasive imaging method for in vivo three-dimensional (3D) characterization of human bone. ${ }^{7}$ Studies with the HRPQCT in finger joints have shown that it has a higher sensitivity for detection of cortical interruptions compared with US, CR, and MRI. ${ }^{8-11}$ Studies that compare HR-pQCT imaging to microCT $(\mu \mathrm{CT})$ imaging with a higher resolution in the detection of cortical interruptions in finger joints are, however, scarce. ${ }^{12}$

The cortical bone in finger joints is thin and low mineralized. Cortical bone regions of approximately $100 \mu \mathrm{m}$ can be observed. ${ }^{13}$ The accuracy of detecting these thin cortices might be lower using HR-pQCT because of its spatial resolution of $130 \mu \mathrm{m} .{ }^{14}$ In regions of thin cortical bone, i.e. in the range of the spatial resolution, the bone density of the cortex will be underestimated due to the partial volume effect. After thresholding the image, this region might appear as a non-bone region (i.e. a false cortical interruption) in the binary image.

Recently, we developed a semi-automated algorithm that reliably detects small cortical interruptions in finger joints on HR-pQCT images. ${ }^{15}$ However, the algorithm uses binary images to detect cortical interruptions, and might therefore be susceptible for false detection of cortical interruptions. Increasing the minimum cut-off diameter of interruptions, might be a solution to avoid false detection of cortical interruptions.

Therefore, the aim of this study was to investigate the reliability of the algorithm on HRpQCT for different minimum diameters of cortical interruptions to detect its presence and to quantify the surface of cortical interruptions compared to $\mu \mathrm{CT}$, as gold standard.

\section{MATERIALS AND METHODS}

\section{Specimens}

For this study, we used anatomic specimens, because $\mu \mathrm{CT}$ imaging can only be executed in-vitro. Metacarpophalangeal (MCP) and proximal interphalangeal (PIP) joints of ten female right hand human anatomic index fingers were imaged by both HR-pQCT and $\mu C T$. One PIP joint could not be evaluated due to a failed $\mu \mathrm{CT}$ scan, due to bad fixation. Thus, 10 MCP and 9 PIP joints remained for analysis. The anatomic specimens were obtained from the department of Anatomy and Embryology, University of Amsterdam, Amsterdam, the Netherlands. A handwritten and signed codicil from each donor posed 
when still alive and well, is kept at the Department of Anatomy and Embryology, University of Amsterdam, Amsterdam, the Netherlands. The medical history of the donors was unknown. The fingers were fixated in formalin.

\section{HR-pQCT and $\mu C T$ image acquisition}

HR-pQCT (XtremeCT, Scanco Medical AG, Switzerland) scans were performed at clinical in vivo settings, i.e. at $60 \mathrm{kVp}$ tube voltage, $900 \mu \mathrm{A}$ tube current, $100 \mathrm{~ms}$ integration time and $82 \mu \mathrm{m}$ voxel size. For the MCP joint, a reference line was placed on top of the metacarpal head, covering an area of $12.00 \mathrm{~mm}$ in proximal direction and $6.04 \mathrm{~mm}$ in distal direction (total scan area $18.04 \mathrm{~mm}, 220$ slices). For the PIP joint, a reference line was placed on top of the proximal phalanx, covering an area of $6.00 \mathrm{~mm}$ in proximal direction and $3.02 \mathrm{~mm}$ in distal direction (total scan area $9.02 \mathrm{~mm}, 110$ slices).

$\mu \mathrm{CT}$ ( $\mu \mathrm{CT}$ 80, Scanco Medical AG, Switzerland) scans were performed with settings: $70 \mathrm{kVp}$ tube voltage, $114 \mu \mathrm{A}$ tube current, $300 \mathrm{~ms}$ integration time and $18 \mu \mathrm{m}$ voxel size. For the MCP joint, a reference line was placed on top of the metacarpal head, covering an area of $10.00 \mathrm{~mm}$ in proximal direction and $5.26 \mathrm{~mm}$ in distal direction (total scan area $15.26 \mathrm{~mm}, 848$ slices). For the PIP joint, a reference line was placed on top of the proximal phalanx, covering an area of $6 \mathrm{~mm}$ in proximal direction and $3.45 \mathrm{~mm}$ in distal direction (total scan area $9.45 \mathrm{~mm}, 525$ slices).

Differences in the extent of the scanned areas as well as in joint angles were noticed because the fingers were scanned horizontally on HR-pQCT and vertically on $\mu \mathrm{CT}$. Corresponding first and last slices of the overlapping region were visually determined to ensure that the same region of interest was used in the detection of cortical interruptions on both imaging modalities. In this overlapping region, the total, cortical and trabecular volumetric bone mineral density (Tot.BMD, Ct.BMD and Tb.BMD, respectively) of the specimens were calculated from the HR-pQCT scans to get insight into the bone mineralization of the specimens.

\section{Cortical interruption detection}

\section{The algorithm}

A semi-automated algorithm for the detection of cortical interruptions was applied on both the HR-pQCT and $\mu \mathrm{CT}$ images. The algorithm is described elsewhere. ${ }^{15}$ In short, first, the outer margin of the cortex was identified according to a modified autocontouring algorithm originally developed for periosteal segmentation of the distal radius and tibia. ${ }^{15-17}$ The contour was visually corrected by an operator if necessary. Second, a binary segmentation into bone and non-bone was performed. For the HRpQCT datasets, the bone was segmented using the standard evaluation protocol from the manufacturer for radius and tibia, which included Laplace-Hamming filtering and thresholding. ${ }^{18}$ Segmentation of the $\mu \mathrm{CT}$ dataset was performed using a Gaussian filtering (sigma $=0.8$, support $=1$ voxel) with a constant threshold of 247 per 1000 of the 
maximum possible voxel value, to equal bone volume to total volume (BV/TV) of the $\mu \mathrm{CT}$ with that of the HR-pQCT. Third, a cortical mask with a constant thickness was generated to identify the cortical bone. This was 4 voxels $(=0.328 \mathrm{~mm})$ for HR-pQCT and 18 voxels $(=0.324 \mathrm{~mm})$ for $\mu \mathrm{CT}$, because this approached the average cortical thickness in MCP joints images. ${ }^{15}$ Fourth, the cortical bone was analyzed for discontinuities that meet the preset criteria of the minimum diameter of a cortical interruption $(>0.16 \mathrm{~mm},>0.33 \mathrm{~mm}$ and $>0.50 \mathrm{~mm}$ ). The total number of detected cortical interruptions and interruption surface per joint were analyzed.

\section{Diameter selection}

The minimum diameter of an interruption was selected by the number of dilation steps. This way, we ensured that the interruption had an opening through the cortex with this minimum diameter and at both the periosteal and endosteal boundary of the cortical mask.

Two-dimensional examples of cortical interruptions on HR-pQCT voxel level and the 5 steps performed by the algorithm are shown in Figure $4.1 \mathrm{I}-\mathrm{V}$. We used sequentially 1 , 2 , and 3 dilation steps resulting in minimum diameters of an interruption of $>0.16 \mathrm{~mm}$ (Figure 4.1a), $>0.33 \mathrm{~mm}$ (Figure 4.1b) and $>0.50 \mathrm{~mm}$ (Figure 4.1c), respectively. Shown is that with the increase in the number of dilation steps, only interruptions with a larger diameter are detected (Figure 4.1-V, green).
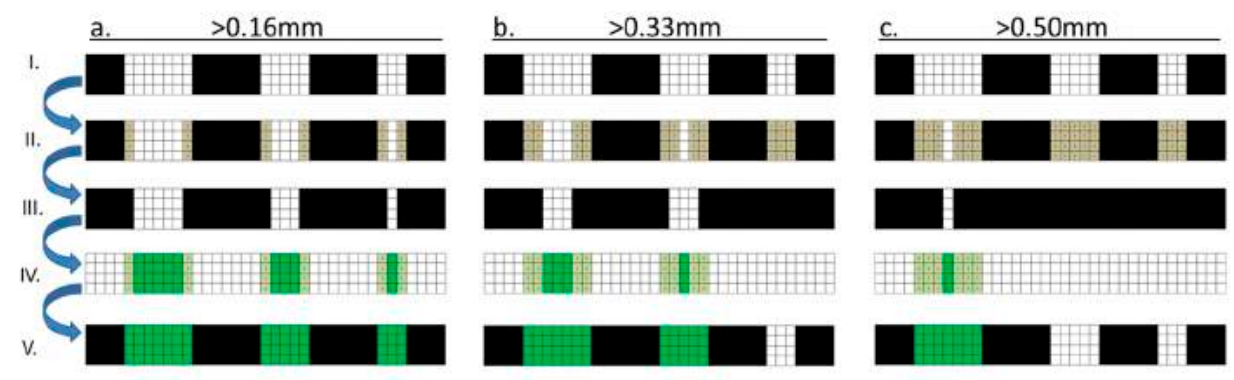

Figure 4.1. Two-dimensional examples of cortical interruptions with different diameters on HR-pQCT voxel level, and the multiple dilation steps that were used to obtain different minimum diameters of interruptions. The following steps are made by the algorithm: (I) shows the original cortex (black) and cortical interruptions (white). The cortex is then dilated with 1 (a), 2 (b) or 3 (c) voxels (II, grey). The dilated cortex (III) is then inverted, and interruptions connected to both the endosteal and periosteal boundary are selected. Finally, the selected interruptions are dilated to its original size (IV), and displayed in the original cortex (V). Shown is that with the increase in the number of dilation steps, only interruptions with a larger diameter are detected $(\mathrm{V}$, green).

For $\mu \mathrm{CT}$ the number of dilations was chosen such that it resulted in similar minimum diameters (Table 4.1). In addition, we were interested if an interruption that was detected with the algorithm on HR-pQCT was present on $\mu \mathrm{CT}$ not taking into account the 
58 $\mid$ Chapter 4

minimum interruption diameter. Moreover, we were interested in how many interruptions would be missed when increasing the minimum diameter on HR-pQCT compared to the total number of interruptions $>0.10 \mathrm{~mm}$ detected on $\mu \mathrm{CT}$. Therefore, for the $\mu \mathrm{CT}$ only, we also set the minimum interruption diameter to $>0.10 \mathrm{~mm}$. All investigated dilation steps and corresponding minimum diameter values on HR-pQCT and $\mu \mathrm{CT}$ are shown in Table 4.1.

Table 4.1. Parameters of the algorithm for the different minimum diameters of the interruptions on HR-pQCT and $\mu \mathrm{CT}$

\begin{tabular}{lcccc}
\hline $\begin{array}{l}\text { Minimum diameter of } \\
\text { the interruption }\end{array}$ & $\begin{array}{c}\text { Number of dilation } \\
\text { steps in } \\
\text { HR-pQCT }\end{array}$ & $\begin{array}{c}\text { Diameter of the } \\
\text { interruptions }\end{array}$ & $\begin{array}{c}\text { Number of dilation } \\
\text { steps in } \\
\mu C T\end{array}$ & $\begin{array}{c}\text { Diameter of the } \\
\text { interruptions }\end{array}$ \\
\hline$>0.10 \mathrm{~mm}$ & - & - & $3(0.054 \mathrm{~mm})$ & $>6(0.108 \mathrm{~mm})$ \\
$>0.16 \mathrm{~mm}$ & $1(0.082 \mathrm{~mm})$ & $>2(0.164 \mathrm{~mm})$ & $5(0.090 \mathrm{~mm})$ & $>10(0.180 \mathrm{~mm})$ \\
$>0.33 \mathrm{~mm}$ & $2(0.164 \mathrm{~mm})$ & $>4(0.328 \mathrm{~mm})$ & $9(0.180 \mathrm{~mm})$ & $>18(0.324 \mathrm{~mm})$ \\
$>0.50 \mathrm{~mm}$ & $3(0.246 \mathrm{~mm})$ & $>6(0.492 \mathrm{~mm})$ & $14(0.252 \mathrm{~mm})$ & $>28(0.504 \mathrm{~mm})$ \\
\hline
\end{tabular}

Minimum diameters of the interruptions are ranging from $0.10 \mathrm{~mm}$ to $0.50 \mathrm{~mm}$ and their corresponding number of dilated voxels on the HR-pQCT and $\mu \mathrm{CT}$ are shown. Values are displayed as number of voxels ( $\mathrm{mm}$ )

\section{Registration of HR-pQCT and $\mu \mathrm{CT}$ images}

To evaluate matching interruptions on exactly the same location on HR-pQCT and $\mu C T$, a $3 \mathrm{D}$ rigid image registration was performed (Figure 4.2). Each bone of the joint was registered separately to avoid registration errors due to different joint angles. The $\mu C T$ images of the interruptions detected were downscaled and transformed to the HR-pQCT images (Figure 4.2-II). The $\mu \mathrm{CT}$ and HR-pQCT images are overlayed, and only interruptions that overlapped with at least 20 voxels $\left(=0.011 \mathrm{~mm}^{3}\right)$ were counted as matching (Figure 4.2-III). This is the smallest interruption volume that can be detected on HR-pQCT images for interruptions $>0.16 \mathrm{~mm}^{15}$

The number of matching interruptions was calculated for the minimum diameters of $>0.16 \mathrm{~mm},>0.33 \mathrm{~mm}$ and $>0.50 \mathrm{~mm}$ on HR-pQCT with a fixed minimum diameter of the interruption of $>0.10 \mathrm{~mm}$ on $\mu \mathrm{CT}$ (matching interruption (m.10mm ) (Figure $4.2 \mathrm{a}$ ). In addition, the number of matching interruptions was calculated for minimum diameters of $>0.16 \mathrm{~mm},>0.33 \mathrm{~mm}$ and $>0.50 \mathrm{~mm}$ on HR-pQCT with the same minimum diameter on $\mu \mathrm{CT}$ (matching interruptions sd $_{\text {) }}$ (Figure 4.2b). 


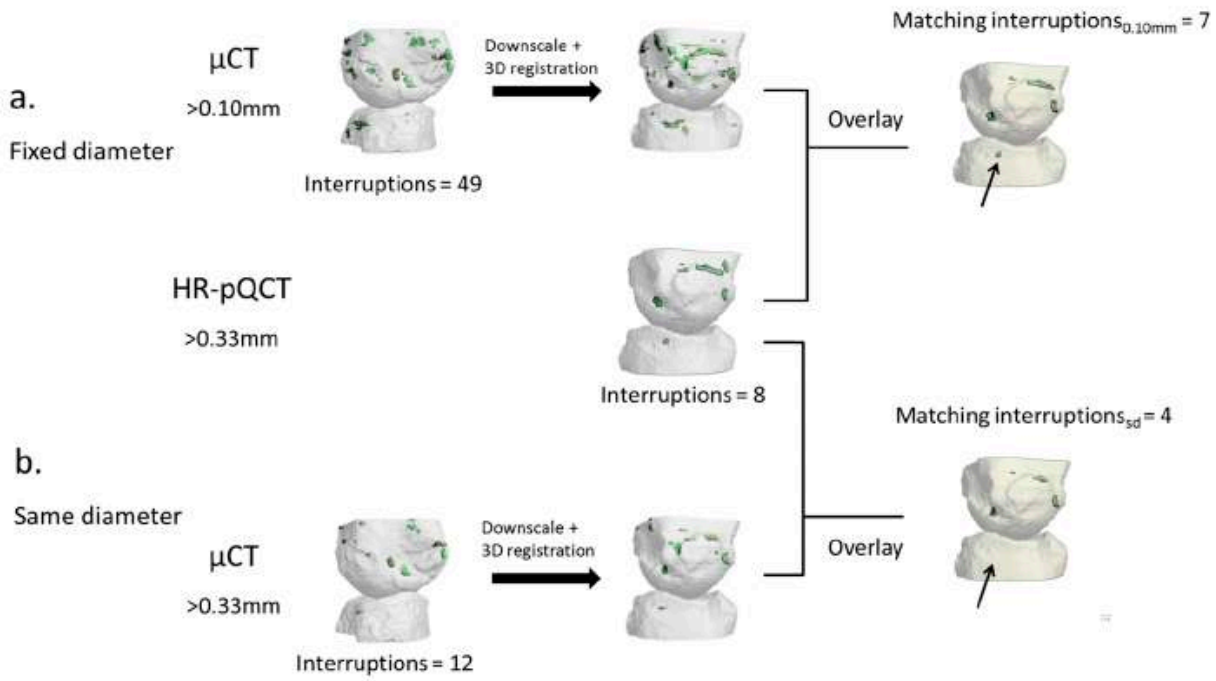

Figure 4.2. Matching interruptions between HR-pQCT and $\mu \mathrm{CT}$ with a variable minimum diameter on HR-pQCT and fixed minimum diameter of $>0.10 \mathrm{~mm}$ on $\mu \mathrm{CT}(\mathrm{a})$, and with the same variable minimum diameter on HRpQCT and $\mu \mathrm{CT}$ (b). (I) Represents the outputs of the cortical interruption algorithm on $\mu \mathrm{CT}$. The $\mu \mathrm{CT}$ image is downscaled and registered to the HR-pQCT output image (II). The $\mu C T$ and HR-pQCT images are overlayed, and interruptions with at least $20 \mathrm{HR}$-pQCT voxels overlap are shown in (III). The black arrow indicates an interruption that was found matching on HR-pQCT and $\mu \mathrm{CT}$ in (a) with a fixed minimum diameter on $\mu \mathrm{CT}$ (matching interruptions $\mathrm{s}_{0.10 \mathrm{~mm}}$ ), but was not found matching with the same minimum diameter $(\mathrm{b}$, matching interruptions $s_{\text {sd }}$ ).

\section{Statistical analysis}

Descriptive statistics were calculated for the number of interruptions and interruption surface per joint. Differences between the imaging modalities were analyzed with Wilcoxon signed-rank test. On the joint level, the reliability of the algorithm for different minimum diameters of the interruptions was estimated by ICC with a two-way random model and absolute agreement.

On the single interruption level, reliability of the HR-pQCT with different minimum diameters was evaluated with positive predictive values (PPV, equation 1) with a fixed $\mu C T$ diameter of $>0.10 \mathrm{~mm}\left(\mathrm{PPV}_{0.10 \mathrm{~mm}}\right)$ and same minimum diameter $\left(\mathrm{PPV}_{\mathrm{sd}}\right)$ of interruptions. In addition, reliability of single interruptions on the HR-pQCT with different minimum diameters were evaluated with the sensitivity (equation 2) for detection of interruptions $>0.10 \mathrm{~mm}$ on $\mu \mathrm{CT}$ (sensitivity $0.10 \mathrm{~mm}$ ), and the sensitivity for detection of interruptions with the same minimum diameter (sensitivity ${ }_{\text {sd }}$ ). Statistical 
$60 \mid$ Chapter 4

analysis was performed using IBM SPSS Statistics for Windows, Version 20.0 (IBM Corp., Armonk, NY).

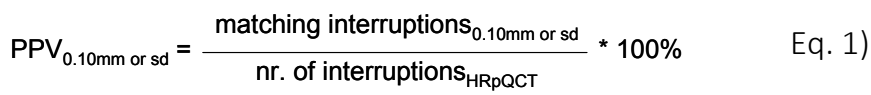

Sensitivity $_{0.10 \mathrm{~mm} \mathrm{or} \mathrm{sd}}=\frac{\text { matching interruptions }_{0.10 \mathrm{~mm} \mathrm{or} \mathrm{sd}}}{\mathrm{nr} \text {. of interruptions }} * 100 \% \quad$ Eq. 2)

\section{RESULTS}

The mean age (SD) of the donors was 85.1 (9.6) years, the medical history was unknown. The average Tot.BMD was $248.5 \mathrm{mgHA} / \mathrm{cm}^{3}$ for the joints. The Ct.BMD and Tb.BMD were 464.0 and $179.7 \mathrm{mgHA} / \mathrm{cm}^{3}$, respectively.

\section{Visual evaluation of HR-pQCT and $\mu \mathrm{CT}$ examinations}

Figure 4.3 shows 3D reconstructions of the same MCP joint when examined with different minimum diameters of the interruptions on HR-pQCT and $\mu \mathrm{CT}$. With both imaging modalities, the cortical bone is very porous and most pores are found on the same location (Figure 4.3a). However, in some, thin cortices can be identified on $\mu \mathrm{CT}$ that are not seen on HR-pQCT (Figure 4.3a, orange arrows).

When applying the algorithm with a cortical interruption diameter of $>0.16 \mathrm{~mm}$ on HR-pQCT and $\mu \mathrm{CT}$, most pores are identified as cortical interruptions and accurately fill the pore in the cortex (Figure 4.3b). Most detected interruptions on HR-pQCT are also detected on $\mu \mathrm{CT}$ when the same diameter of $>0.16 \mathrm{~mm}$ is applied (Figure $4.3 b$, green arrows), with some exceptions (Figure $4.3 b$, red arrows).

When a minimum cortical interruption diameter of $>0.33 \mathrm{~mm}$ is chosen on HR-pQCT and $\mu \mathrm{CT}$, only larger pores are identified as cortical interruptions (Figure 4.3c). Again, most interruptions detected on HR-pQCT are also detected on $\mu \mathrm{CT}$ (Figure 4.3c, green arrows), with some discrepancies (Figure 4.3c, red arrows).

Interestingly, the majority of interruptions with a diameter of $>0.50 \mathrm{~mm}$ on HR-pQCT are not identified as interruptions of the same size on $\mu \mathrm{CT}$ (Figure $4.3 d$, red arrows).

When applying the algorithm with a cortical interruption diameter of $>0.10 \mathrm{~mm}$ on $\mu \mathrm{CT}$, even smaller pores are identified as interruptions (Figure 4.3e, green arrows). When comparing interruptions with a diameter of $>0.16 \mathrm{~mm},>0.33 \mathrm{~mm}$ and $>0.50 \mathrm{~mm}$ on HR-pQCT (Figure 4.3b-d) to the interruptions with a diameter of $>0.10 \mathrm{~mm}$ on $\mu \mathrm{CT}$ (Figure 4.3e), we can see that almost all interruptions detected on HR-pQCT are also detected on $\mu \mathrm{CT}$, although the majority has a smaller diameter (Figure 4.3e). 
HR-pQCT

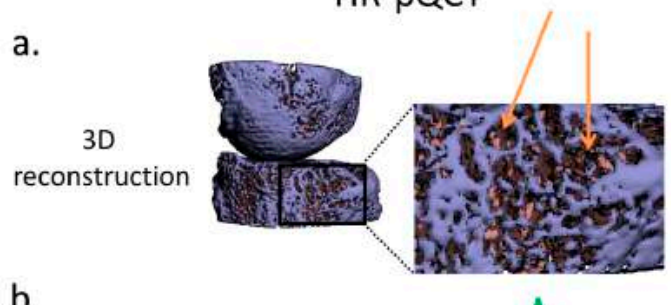

b.

C.

d.

e.
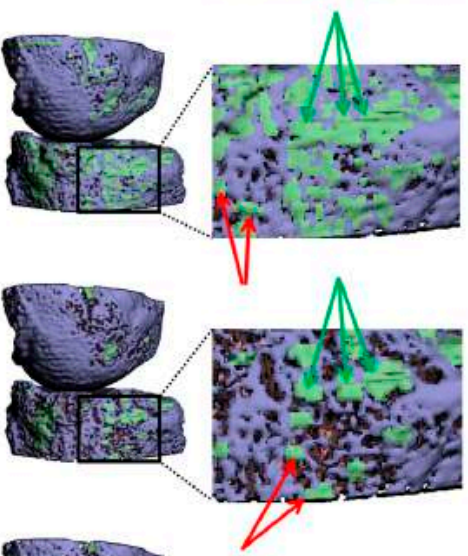

$>0.50 \mathrm{~mm}$
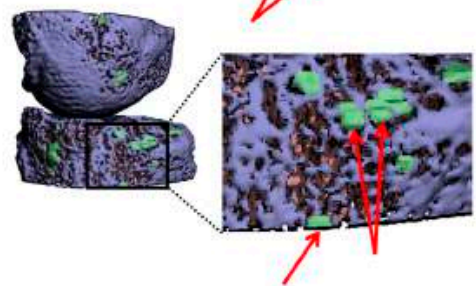

$>0.10 \mathrm{~mm}$ $\mu \mathrm{CT}$
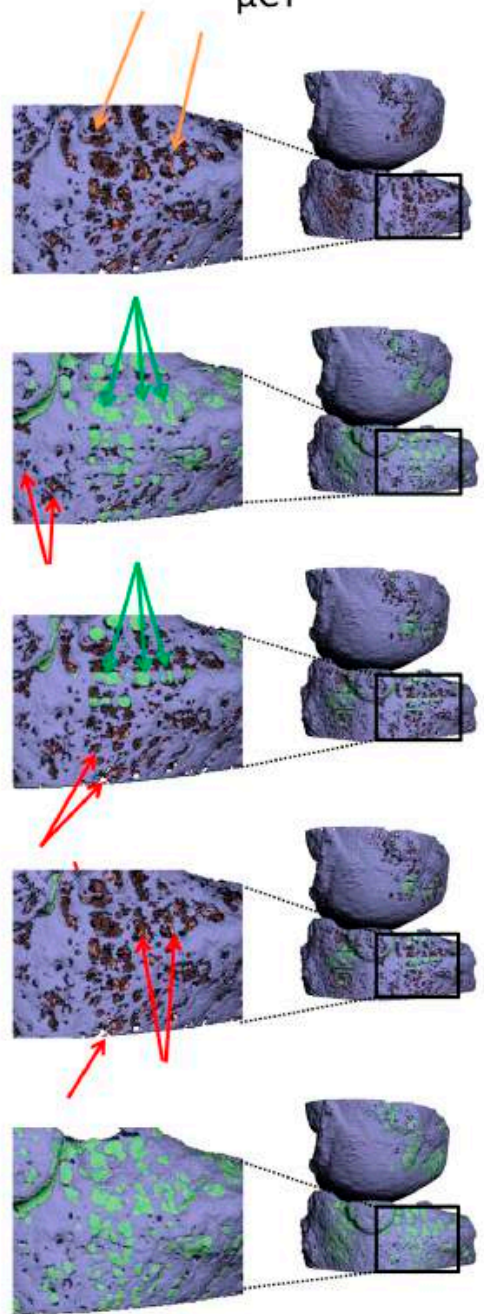

Figure 4.3. Typical example of 3D reconstructions of an MCP joint of a HR-pQCT scan (left) and $\mu C T$ scan (right) and the 3D outputs of the algorithm for the different minimum diameters of the interruptions. The cortical region is indicated in purple, trabecular region in orange, and the cortical interruptions that were detected by the algorithm are shown in green. a) Shows that the cortex is very porous on the HR-pQCT and $\mu C T$ scan, and that thin cortices were identified on $\mu \mathrm{CT}$, but not on HR-pQCT (orange arrows). b) Shows that most pores were identified as cortical interruptions on HR-pQCT and $\mu \mathrm{CT}$. In addition, most cortical interruptions detected on HR-pQCT were also detected on $\mu \mathrm{CT}$ (green arrows), with some exceptions (red arrows). c) Shows that only larger pores are identified as cortical interruptions when a minimum interruption diameter of $>0.33 \mathrm{~mm}$ is chosen. Again, most cortical interruptions detected on HR-pQCT were also detected on $\mu \mathrm{CT}$ (green arrows), with some exceptions (red arrows). d) Shows that large cortical interruptions detected on HR-pQCT were not identified as large interruptions on $\mu \mathrm{CT}$ (red arrows). e) Shows that even smaller pores are identified as cortical interruptions when a minimum interruption diameter $>0.10 \mathrm{~mm}$ was chosen (green arrows). 
$62 \mid$ Chapter 4

\section{Reliability at the joint level for different diameters of the interruptions}

Table 4.2 shows that the mean number of interruptions detected per joint depends on the diameter cut-off applied and ranges from 3.4 to 53.5 on HR-pQCT and from 1.8 to 45.1 on $\mu \mathrm{CT}$ for interruptions $>0.50 \mathrm{~mm}$ and $>0.16 \mathrm{~mm}$, respectively. Furthermore, it can be seen that the number of cortical interruptions per joint differs significantly between HR-pQCT and $\mu \mathrm{CT}$ for all diameter cut-offs (Table 4.2). The interruption surface per joint only differs significantly for interruptions with a diameter of $>0.50 \mathrm{~mm}$ (Table 4.2).

For the number of interruptions and interruption surface, almost perfect reliability (ICC $\geq 0.81$ ) at the joint level between HR-pQCT and $\mu \mathrm{CT}$ is found for interruptions with a diameter of $>0.16 \mathrm{~mm}$ and $>0.33 \mathrm{~mm}$. For large interruptions $(>0.50 \mathrm{~mm})$, a fair to moderate reliability is found (ICC 0.21 - 0.52) (Table 4.2).

Table 4.2. The number of cortical interruptions and interruption surface per joint detected on HR-pQCT and $\mu \mathrm{CT}$

\begin{tabular}{|c|c|c|c|c|c|c|c|c|}
\hline \multirow{2}{*}{$\begin{array}{l}\text { Minimum } \\
\text { diameter of } \\
\text { the } \\
\text { interruption }\end{array}$} & \multicolumn{2}{|c|}{ Number of interruptions } & \multirow[t]{2}{*}{$p$-value } & \multirow[t]{2}{*}{ ICC } & \multicolumn{2}{|c|}{ Interruption surface } & \multirow[t]{2}{*}{$p$-value } & \multirow[t]{2}{*}{ ICC } \\
\hline & HR-pQCT & $\mu \mathrm{CT}$ & & & HR-pQCT & $\mu \mathrm{CT}$ & & \\
\hline$>0.16 \mathrm{~mm}$ & $\begin{array}{c}53.5 \\
(33.9)\end{array}$ & $\begin{array}{c}45.1 \\
(30.4)\end{array}$ & 0.01 & $\begin{array}{c}0.91 \\
(0.65-0.97)\end{array}$ & $\begin{array}{c}44.6 \\
(37.8) \mathrm{mm}^{2}\end{array}$ & $\begin{array}{c}46.3 \\
(42.6) \mathrm{mm}^{2}\end{array}$ & 0.60 & $\begin{array}{c}0.93 \\
(0.82-0.97)\end{array}$ \\
\hline$>0.33 \mathrm{~mm}$ & $\begin{array}{c}9.5 \\
(9.1)\end{array}$ & $\begin{array}{c}12.8 \\
(11.7)\end{array}$ & 0.02 & $\begin{array}{c}0.81 \\
(0.52-0.92)\end{array}$ & $\begin{array}{c}13.5 \\
(14.5) \mathrm{mm}^{2}\end{array}$ & $\begin{array}{c}11.7 \\
(12.9) \mathrm{mm}^{2}\end{array}$ & 0.75 & $\begin{array}{c}0.86 \\
(0.67-0.94)\end{array}$ \\
\hline$>0.50 \mathrm{~mm}$ & $\begin{array}{c}3.4 \\
(4.1)\end{array}$ & $\begin{array}{c}1.8 \\
(2.2)\end{array}$ & 0.02 & $\begin{array}{c}0.52 \\
(0.11-0.78)\end{array}$ & $\begin{array}{c}6.5 \\
(8.4) \mathrm{mm}^{2}\end{array}$ & $\begin{array}{c}1.6 \\
(2.0) \mathrm{mm}^{2}\end{array}$ & $<0.01$ & $\begin{array}{c}0.21 \\
(-0.15-0.56)\end{array}$ \\
\hline
\end{tabular}

Values are displayed as mean (standard deviation) and for ICC as value ( $95 \%$ confidence interval), ICC $=$ intraclass correlation coefficient.

\section{Reliability at the level of a single interruption}

\section{Fixed $\mu \mathrm{CT}$ diameter of $>0.10 \mathrm{~mm}$}

At the level of a single interruption, the $P P V_{0.10 m m}$ is $84.9 \%$ for interruptions with a diameter of $>0.16 \mathrm{~mm}, 96.7 \%$ for interruptions with a diameter of $>0.33 \mathrm{~mm}$, and $100 \%$ for interruptions with a diameter of $>0.50 \mathrm{~mm}$. The sensitivity $0.10 \mathrm{~mm}$ is $62.4 \%$ for interruptions with a diameter of $>0.16 \mathrm{~mm}, 12.7 \%$ for interruptions with a diameter of $>0.33 \mathrm{~mm}$, and $4.7 \%$ for interruptions with a diameter $>0.50 \mathrm{~mm}$.

\section{Same minimum diameter}

In addition, the $\mathrm{PPV}_{\text {sd }}$ is substantial for interruptions with a diameter of $>0.16 \mathrm{~mm}$ and $>0.33 \mathrm{~mm}\left(\mathrm{PPV}_{\mathrm{sd}}=66.6 \%\right.$ and $66.3 \%$, respectively), but fair for interruptions with $\mathrm{a}$ diameter of $>0.50 \mathrm{~mm}\left(\mathrm{PPV}_{\mathrm{sd}}=38.5 \%\right)$. The sensitivity sd was substantial for interruptions with a diameter of $>0.16 \mathrm{~mm}$ and $>0.50 \mathrm{~mm}$ (Sensitivity sd $=79.1 \%$ and $73.5 \%$, 
\begin{tabular}{l|l} 
Reliability of algorithm on HR-pQCT compared to microCT & \\
63
\end{tabular}

respectively), and moderate for interruptions with a diameter of $>0.33 \mathrm{~mm}$ (Sensitivity sd $_{\text {d }}$ $=49.4 \%$ ).

\section{DISCUSSION}

In this study, we investigated the reliability of cortical interruptions detected with our semi-automated algorithm on HR-pQCT in comparison to $\mu \mathrm{CT}$ as gold standard. We showed that almost all cortical interruptions detected with the algorithm on HR-pQCT were also detected on $\mu \mathrm{CT}$ on the same location. However, the size of an interruption detected on HR-pQCT can be different from that on $\mu C T$. In regions of thin bone, the HRpQCT might not be able to identify these thin structures and can therefore overestimate the interruption diameter (Figure 4.1a, orange arrows). ${ }^{14}$ In regions of dense cortical bone, the HR-pQCT tends to underestimate the interruption diameter due to the lower resolution. ${ }^{19,20}$ On the joint level, almost perfect agreement for the number of interruptions and interruption surface was obtained for interruptions with a minimum diameter of $>0.16 \mathrm{~mm}$ and $>0.33 \mathrm{~mm}$. As expected, the $P P V_{0.10 \mathrm{~mm}}$ of the detection of a single interruption improved with increasing diameter, and was excellent for $>0.33 \mathrm{~mm}$ and $>0.50 \mathrm{~mm}\left(\mathrm{PPV}_{0.10 \mathrm{~mm}} \geq 97 \%\right.$ ). However, this improvement in $\mathrm{PPV}_{0.10 \mathrm{~mm}}$ comes with the expense of a lower sensitivity $y_{0.10 \mathrm{~mm}}$, which was low for interruptions with a minimum diameter $>0.33 \mathrm{~mm}$ and $>0.50 \mathrm{~mm}$ ( sensitivity $_{0.10 \mathrm{~mm}} \leq 12.7 \%$ ).

The number of interruptions with a minimum diameter of $>0.16 \mathrm{~mm}$ and interruption surface per joint detected in the present study was substantially higher than observed in a previous study by our study team (53.5 and $44.6 \mathrm{~mm}^{2}$ versus 25.0 and $18.9 \mathrm{~mm}^{2}$ ). ${ }^{15}$ This can be explained by the relatively high mean age of the donors in the present study (85.1 years) compared to the subjects investigated in our previous study (48.8 years). ${ }^{15}$ Due to the old age of the donors and preservation in formalin, the cortices become less mineralized. ${ }^{21}$ The Tot.BMD of these specimens was about $25 \%$ lower than the average in the normal population $\left(248.5 \mathrm{mgHA} / \mathrm{cm}^{3}\right.$ versus $327 \mathrm{mgHA} / \mathrm{cm}^{3}$, respectively). ${ }^{13}$ In addition, the Ct.BMD and Tb.BMD were respectively 31\% and 22\% lower compared to the normal population (464.0 and $179.7 \mathrm{mgHA} / \mathrm{cm}^{3}$ versus 593 and $262 \mathrm{mgHA} / \mathrm{cm}^{3}$, respectively). ${ }^{13}$ These low mineralized and thin cortices are more likely to represent nonbone voxels after binary segmentation than a high mineralized cortex, leading to more cortical interruptions.

Bone density loss is also observed in patients with RA, thus potential overestimation of the number of interruptions and its size might also be observed in patients with RA. However, the Tot.BMD, Ct.BMD and Tb.BMD in patients with RA were 5-7\% lower than in the normal population (308, 566 and $243 \mathrm{mgHA} / \mathrm{cm}^{3}$ versus 327, 593 and $262 \mathrm{mgHA} / \mathrm{cm}^{3}$, respectively). ${ }^{13}$ Meaning that potential overestimation is possible in patients with RA compared to healthy controls, but it is not likely to have a large effect, because of the small difference in BMD. 
A limitation of our algorithm is that it uses a cortical mask with a constant thickness. The chosen value fitted best with the average cortical thickness in the joint. Although this approach is robust, it introduces a systematic error: a cortical interruption is only detected if non-bone voxels connect the inner and outer surface of the mask with a chosen amount of voxels dependent on the number of dilation steps. In regions where the cortex is thin, part of the underlying fatty bone marrow might be included in the mask as non-bone voxels and subsequently become part of a cortical interruption. This might lead to overestimation of the size of an interruption detected. On the other hand, regions of relatively thick cortical bone will lead to underestimation of the interruption size.

The current investigation of the reliability of cortical interruptions detected with our semi-automated algorithm on HR-pQCT compared to $\mu \mathrm{CT}$ as gold standard is a next step in the assessment of small cortical interruptions in finger joints by HR-pQCT. The algorithm can best be used for the detection of interruptions with a minimum diameter of $>0.16 \mathrm{~mm}$ or $>0.33 \mathrm{~mm}$ on HR-pQCT, because an almost perfect agreement on the number and surface of interruptions with $\mu \mathrm{CT}$ was obtained at the joint level, and the locations of interruptions detected on HR-pQCT well matched with $\mu \mathrm{CT}$. The choice can depend on the scope of the study, if a higher PPV is preferred, one should consider using interruptions $>0.33 \mathrm{~mm}$, if a higher sensitivity is preferred, one should consider using interruptions $>0.16 \mathrm{~mm}$.

A next step in the validation of the algorithm is the investigation of the reproducibility of the algorithm on patient scan/re-scan (repositioning) data. Additionally, in patients with RA, loss of cortical bone (i.e. a cortical interruption) is often accompanied with loss of trabeculae. ${ }^{1}$ Therefore, it would be interesting to extend the algorithm with determination of trabecular bone voids adjacent to the cortical interruption. Finally, the algorithm could be used in clinical studies in order to determine its potential value in monitoring patients with RA, and discriminating patients with RA early in the disease from healthy controls. The average interruption diameter found in previous studies in patients with RA was $2.2 \mathrm{~mm} .^{22,23}$ Small interruptions $(<0.50 \mathrm{~mm})$ were found in both healthy controls and patients with $\mathrm{RA}^{8}$, and interruptions $>1.9 \mathrm{~mm}$ were found highly specific for patients with RA. ${ }^{8}$ In early detection of cortical bone loss in patients with RA, this algorithm has the advantage of including the detection of these small cortical interruptions $(<0.50 \mathrm{~mm})$ in a highly reliable manner in comparison to visual scoring which showed fair to moderate reliability. ${ }^{12}$

In conclusion, this semi-automated algorithm for detection of cortical interruptions with HR-pQCT in finger joints performed best for interruptions with a minimum diameter of $>0.16 \mathrm{~mm}$ or $>0.33 \mathrm{~mm}$. Almost perfect reliability was obtained at the joint level and the interruptions matched well with those detected on $\mu \mathrm{CT}$. This algorithm can be useful for evaluation of cortical interruptions in rheumatic diseases. 
Reliability of algorithm on HR-pQCT compared to microCT $\mid$

\section{REFERENCES}

1. Schett G, Gravallese E. Bone erosion in rheumatoid arthritis: mechanisms, diagnosis and treatment. Nature reviews Rheumatology. 2012;8(11):656-64.

2. Geusens P, van den Bergh J. Bone erosions in rheumatoid arthritis. Rheumatology. 2014;53(1):4-5.

3. Baillet A, Gaujoux-Viala C, Mouterde G, Pham T, Tebib J, Saraux A, et al. Comparison of the efficacy of sonography, magnetic resonance imaging and conventional radiography for the detection of bone erosions in rheumatoid arthritis patients: a systematic review and meta-analysis. Rheumatology. 2011; 50(6):1137-47.

4. Dohn UM, Ejbjerg BJ, Hasselquist M, Narvestad E, Moller J, Thomsen HS, et al. Detection of bone erosions in rheumatoid arthritis wrist joints with magnetic resonance imaging, computed tomography and radiography. Arthritis Res Ther. 2008;10(1):R25.

5. Geusens P, Chapurlat R, Schett G, Ghasem-Zadeh A, Seeman E, de Jong J, et al. High-resolution in vivo imaging of bone and joints: a window to microarchitecture. Nat Rev Rheumatol. 2014; 10(5):304-13.

6. Ejbjerg B, Narvestad E, Rostrup E, Szkudlarek M, Jacobsen S, Thomsen HS, et al. Magnetic resonance imaging of wrist and finger joints in healthy subjects occasionally shows changes resembling erosions and synovitis as seen in rheumatoid arthritis. Arthritis Rheum. 2004;50(4):1097-106.

7. Boutroy S, Bouxsein ML, Munoz F, Delmas PD. In vivo assessment of trabecular bone microarchitecture by high-resolution peripheral quantitative computed tomography. J Clin Endocrinol Metab. 2005;90(12): 6508-15.

8. Stach CM, Bauerle M, Englbrecht M, Kronke G, Engelke K, Manger B, et al. Periarticular bone structure in rheumatoid arthritis patients and healthy individuals assessed by high-resolution computed tomography. Arthritis Rheum. 2010;62(2):330-9.

9. Srikhum W, Virayavanich W, Burghardt AJ, Yu A, Link TM, Imboden JB, et al. Quantitative and semiquantitative bone erosion assessment on high-resolution peripheral quantitative computed tomography in rheumatoid arthritis. J Rheumatol. 2013;40(4):408-16.

10. Albrecht A, Finzel S, Englbrecht M, Rech J, Hueber A, Schlechtweg $P$, et al. The structural basis of MRI bone erosions: an assessment by microCT. Ann Rheum Dis. 2013;72(8):1351-7.

11. Finzel S, Ohrndorf S, Englbrecht M, Stach C, Messerschmidt J, Schett G, et al. A detailed comparative study of high-resolution ultrasound and micro-computed tomography for detection of arthritic bone erosions. Arthritis Rheum. 2011;63(5):1231-6.

12. Scharmga A, Peters M, van Tubergen A, van den Bergh J, de Jong J, Loeffen D, et al. Visual detection of cortical breaks in hand joints: reliability and validity of high-resolution peripheral quantitative CT compared to microCT. BMC Musculoskelet Disord. 2016;17(1):271.

13. Fouque-Aubert A, Boutroy S, Marotte H, Vilayphiou N, Bacchetta J, Miossec P, et al. Assessment of hand bone loss in rheumatoid arthritis by high-resolution peripheral quantitative CT. Ann Rheum Dis. 2010;69(9):1671-6.

14. Krause M, Museyko O, Breer S, Wulff B, Duckstein C, Vettorazzi E, et al. Accuracy of trabecular structure by HR-pQCT compared to gold standard muCT in the radius and tibia of patients with osteoporosis and long-term bisphosphonate therapy. Osteoporos Int. 2014;25(5):1595-606.

15. Peters M, de Jong J, Scharmga A, van Tubergen A, Loeffen D, Weijers R, et al. The reproducibility of a semi-automatic algorithm in the detection of cortical breaks and adjacent trabecular bone loss in scanrescan data from patients with early arthritis [abstract]. Arthritis Rheum. 2016;68 (suppl 10).

16. Burghardt AJ, Buie HR, Laib A, Majumdar S, Boyd SK. Reproducibility of direct quantitative measures of cortical bone microarchitecture of the distal radius and tibia by HR-pQCT. Bone. 2010;47(3):519-28.

17. Buie HR, Campbell GM, Klinck RJ, MacNeil JA, Boyd SK. Automatic segmentation of cortical and trabecular compartments based on a dual threshold technique for in vivo micro-CT bone analysis. Bone. 2007;41(4):505-15.

18. Laib A, Hauselmann HJ, Ruegsegger P. In vivo high resolution 3D-QCT of the human forearm. Technol Health Care. 1998;6(5-6):329-37.

19. Tjong W, Kazakia GJ, Burghardt AJ, Majumdar S. The effect of voxel size on high-resolution peripheral computed tomography measurements of trabecular and cortical bone microstructure. Med Phys. 2012; 39(4):1893-903. 
20. Tjong W, Nirody J, Burghardt AJ, Carballido-Gamio J, Kazakia GJ. Structural analysis of cortical porosity applied to HR-pQCT data. Med Phys. 2014;41(1):013701.

21. Zebaze RM, Ghasem-Zadeh A, Bohte A, Iuliano-Burns S, Mirams M, Price RI, et al. Intracortical remodelling and porosity in the distal radius and post-mortem femurs of women: a cross-sectional study. Lancet. 2010;375(9727):1729-36.

22. Finzel S, Rech J, Schmidt S, Engelke K, Englbrecht M, Schett G. Interleukin-6 receptor blockade induces limited repair of bone erosions in rheumatoid arthritis: a micro CT study. Ann Rheum Dis. 2013;72(3): 396-400.

23. Finzel S, Rech J, Schmidt S, Engelke K, Englbrecht M, Stach C, et al. Repair of bone erosions in rheumatoid arthritis treated with tumour necrosis factor inhibitors is based on bone apposition at the base of the erosion. Ann Rheum Dis. 2011;70(9):1587-93. 


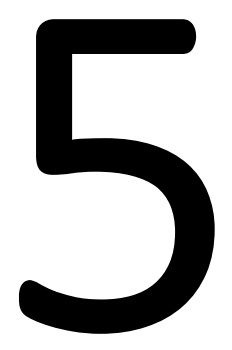

An automated algorithm for the detection of cortical interruptions and its underlying loss of trabecular bone; a reproducibility study

M. Peters, J. de Jong, A. Scharmga, A. van Tubergen, P. Geusens, D. Loeffen, R. Weijers, S.K. Boyd, C. Barnabe, K. S. Stok, B. van Rietbergen, J. van den Bergh 


\section{ABSTRACT}

\section{Background}

We developed a semi-automated algorithm that detects cortical interruptions in finger joints using high-resolution peripheral quantitative computed tomography (HR-pQCT), and extended it with trabecular void volume measurement. In this study we tested the reproducibility of the algorithm using scan/re-scan data.

\section{Methods}

Second and third metacarpophalangeal joints of 21 subjects (mean age 49 (SD 11) years, 17 early rheumatoid arthritis and 4 undifferentiated arthritis, all diagnosed $<1$ year ago) were imaged twice by HR-pQCT on the same day with repositioning between scans. The images were analyzed twice by one operator (OP1) and once by an additional operator (OP2), who independently corrected the bone contours when necessary. The number, surface area and volume of interruptions per joint were obtained. Intra- and interoperator reliability and intra-operator reproducibility were determined by intra-class correlation coefficients (ICC). Intra-operator reproducibility errors were determined as the least significant change ( $\left.\mathrm{LSC}_{\mathrm{SD}}\right)$.

\section{Results}

Per joint, the mean number of interruptions was 3.1 (SD 3.6), mean interruption surface area 4.2 (SD 7.2) $\mathrm{mm}^{2}$, and mean interruption volume 3.5 (SD 10.6) $\mathrm{mm}^{3}$ for OP1. Intraand inter-operator reliability was excellent for the cortical interruption parameters (ICC $\geq 0.91$ ), except good for the inter-operator reliability of the interruption surface area (ICC 0.70). The LSC SD per joint was 4.2 for the number of interruptions, $5.8 \mathrm{~mm}^{2}$ for interruption surface area, and $3.2 \mathrm{~mm}^{3}$ for interruption volume.

\section{Conclusion}

The algorithm was highly reproducible in the detection of cortical interruptions and their volume. Based on the LSC findings, the potential value of this algorithm for monitoring structural damage in the joints in early arthritis patients needs to be tested in clinical studies. 
\begin{tabular}{l|l} 
& \\
Reproducibility of an automated algorithm & 69
\end{tabular}

\section{BACKGROUND}

Rheumatoid arthritis (RA) is a chronic disease, in which inflammation at the joint may lead to erosions (i.e. cortical interruptions). ${ }^{1,2}$ Interruptions in the cortical bone surface are often accompanied with underlying trabecular bone loss. ${ }^{3-5}$ The presence, size and number of cortical interruptions within a joint, and the number of joints affected, are each associated with poor functional outcome and predictors of further progression of structural damage. ${ }^{3,6,7}$ The quantification of interruptions on conventional radiography (CR) is considered the gold standard in clinical practice, however, it has a lower sensitivity compared to ultrasound, computed tomography (CT) and magnetic resonance imaging (MRI). ${ }^{8-10}$

High-resolution peripheral quantitative CT (HR-pQCT) is a low-dose imaging technique that is able to assess the three-dimensional (3D) bone structure at the micro-scale ( $82 \mu \mathrm{m}$ nominal isotropic voxel size) of the peripheral skeleton in vivo. ${ }^{11}$ Multiple studies have reported results on the visual inspection of the presence, number and size of interruptions with underlying trabecular bone voids in finger joints of patients with RA using HR-pQCT. ${ }^{12-22}$ Excellent intra- and inter-rater reliability have been reported, but in all these studies only relatively large cortical interruptions were scored (mean diameter $>1.5 \mathrm{~mm}$ ). ${ }^{5,13-15,21,23,24}$ In an earlier study, we showed that the inter-operator reliability is fair when visually scoring smaller cortical interruptions. ${ }^{25}$

In addition, the quantification of interruption volume was primarily based on simple distance measures on a two-dimensional (2D) slice. ${ }^{5,14,15,17,18,23}$ A more extensive method is the 3D automated volume determination developed by Töpfer et al.. ${ }^{21}$ However, in this method the location of the interruption still has to be visually identified by an operator. In addition, this volume determination was performed in large interruptions (average volume: $9.3 \mathrm{~mm}^{3}$ ).

We have therefore developed a semi-automated algorithm that reliably detects small cortical interruptions (with a diameter $\geq 0.246 \mathrm{~mm}$ ). ${ }^{26}$ In addition, we showed that interruptions with a diameter of $\geq 0.41 \mathrm{~mm}$ detected on HR-pQCT were also detected on $\mu \mathrm{CT}$, the $3 \mathrm{D}$ gold standard. ${ }^{27}$ However, this algorithm only analyzed the presence of an interruption in the cortex and did not consider the underlying trabecular bone loss as part of the total interruption volume. This is important because not only the presence but also the size of cortical interruptions (which includes the trabecular bone void) are associated with poor functional outcome and predictors of further progression of structural damage. ${ }^{3,6,7}$

Furthermore, the reproducibility of our algorithm on scan/re-scan with repositioning inbetween the scans has not yet been tested in the standard workflow of the HR-pQCT scanner. Only the effect of the operator was investigated and not the influence of repositioning of the hand nor the effect of image quality (noise and motion artifacts) in addition to the effect of the operator. Two previous studies tested the reproducibility on scan/re-scan data in the standard workflow of the HR-pQCT scanner for structural and density parameters in metacarpal heads. ${ }^{13,28}$ The density parameters showed precision 
$70 \mid$ Chapter 5

errors of $\leq 2 \%$, but for trabecular and cortical structural parameters, precision errors up to $33 \%$ were found. ${ }^{13,28}$ However, precision errors of the cortical parameters were only tested in healthy controls and not in early arthritis patients. Moreover, the phalangeal base was not included as part of the metacarpophalangeal (MCP) joint.

Therefore, the aims of this study were: 1) to extend our algorithm for detection of cortical interruptions with underlying trabecular bone void volume detection, 2) to evaluate the precision errors of our algorithm to detect cortical interruptions and its volume using scan/re-scan data in the standard workflow of the HR-pQCT scanner, and 3) to evaluate the precision errors of the trabecular and cortical density and microstructure parameters in the standard workflow of the HR-pQCT scanner..

\section{MATERIALS AND METHODS}

\section{Patient population}

Twenty-one patients were recruited (mean age 49 (SD 11) years) with early RA ( $n=17$ ) and undifferentiated arthritis $(n=4)$, all diagnosed $<1$ year from the Early Inflammatory Arthritis Clinics of the Division of Rheumatology at the University of Calgary, Canada. All patients with early RA fulfilled the 2010 American College of Rheumatology (ACR)/European League Against Rheumatism (EULAR) classification criteria for RA. ${ }^{29}$ Ethical approval was obtained from the Conjoint Health Research Ethics Board at the University of Calgary, Canada (REB 15-0582). All participants signed informed consent.

\section{HR-pQCT scanning procedure}

The second and third metacarpophalangeal joints of the dominant hand were scanned twice with HR-pQCT (XtremeCT, Scanco Medical AG, Switzerland) using the image acquisition protocol developed by the Study group for xtrEme Computed Tomography in RA (SPECTRA), an international collaboration of HR-pQCT users. $^{30}$ Scanning was performed at clinical in vivo settings, i.e. at $60 \mathrm{kVp}$ tube voltage, $900 \mu \mathrm{A}$ tube current, $100 \mathrm{~ms}$ integration time and images were reconstructed using an $82 \mu \mathrm{m}$ nominal isotropic voxel size. The reference line was placed at the midpoint of the concave articular surface at the base of the second or third proximal phalanx (whichever was the most distal of the two). The scan covered a length of $9.02 \mathrm{~mm}$ (1 stack) in the distal direction and $18.04 \mathrm{~mm}$ ( 2 stacks) in the proximal direction (total scan length $27.06 \mathrm{~mm}$, 330 slices, 3 stacks) (Supplementary Figure S5.1). The total scanning time was approximately 9 minutes. After the first scan, the patients removed their hand from the stabilization platform, rested for five minutes, and were then repositioned for a second scan. Subject scans were evaluated on motion artifacts per stack according to Pialat et

al.. ${ }^{31}$ Stacks of poor quality (grade $>3$ ) on the first and/or second scan were excluded from further analyses. ${ }^{31}$ 
Reproducibility of an automated algorithm $\mid$

Image analysis

\section{Outer contour}

The outer margin of the cortex was identified using a modified previously described auto-contouring algorithm for periosteal segmentation of the distal radius and tibia. ${ }^{26}$ For the first scans, the contours were visually inspected and, if necessary, corrected by one operator twice (MP, OP1) with three years of experience with HR-pQCT to enable calculation of intra-operator reliability. Contouring of the first scans was performed by one additional operator (JdJ, OP2) with five years of experience with HR-pQCT to enable calculation of inter-operator reliability. For the second scans, the contours were corrected by OP1, to enable calculation of intra-operator reproducibility. The number of manual corrections largely depends on the presence and severity of motion artifacts and the number of large cortical interruptions $\left(>1 \mathrm{~mm}^{2}\right)$ within the joint. Corrections are always necessary in case of large cortical interruptions and usually necessary with motion grades $\geq 3$. Figure 5.1 shows two examples of contours that were corrected by the operators, one due to motion artifacts (Figure 5.1a), and another due to a large cortical interruption (Figure 5.1b).

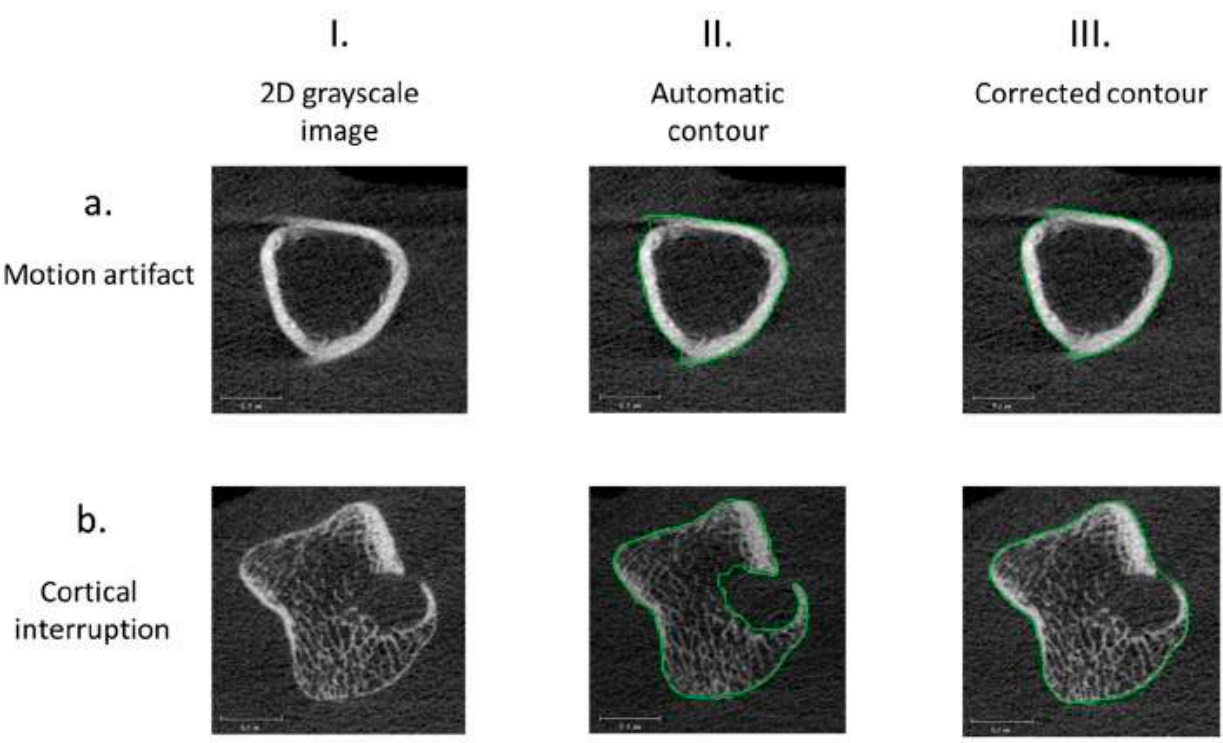

Figure 5.1. Typical examples of 2D grayscale images in which the contour is manually corrected by the operators. (a) Due to a motion artifact, the automatically obtained contour was not tight around its original structure (a. II). The operators corrected this (a. III). In b) a large cortical interruption with underlying trabecular bone loss is shown (b. I, arrow). The automatically obtained contour does not follow the outer margin of the original structure at the cortical interruption (b. II). The operators therefore corrected the contour (b. III). 
$72 \mid$ Chapter 5

Binary image, and bone structure and density parameters

The outer contours obtained from OP1 and OP2 were used for standard and cortical evaluation protocols. ${ }^{11,32}$ The cortical evaluation protocol incorporates the detection of the inner cortical contour as explained by Burghardt et al.. ${ }^{32}$ No corrections were applied to the inner cortical contour. The standard evaluation protocol from the manufacturer (Scanco Medical AG, Brüttisellen, Switzerland) for radius and tibia, which included Laplace-Hamming filtering and thresholding, ${ }^{33}$ was used to distinguish bone from nonbone voxels to create a 3D binary image, and to determine the bone density and microarchitectural parameters as described elsewhere. ${ }^{11}$ From the standard evaluation protocol, the volumetric bone mineral density (VBMD) $\left[\mathrm{mg} \mathrm{HA} / \mathrm{cm}^{3}\right.$ ] in the total (Tot.BMD) and trabecular (Tb.BMD) region was obtained. ${ }^{11}$ Furthermore, trabecular number (Tb.N) $\left[\mathrm{mm}^{-1}\right]$, thickness (Tb.Th) $[\mu \mathrm{m}]$, separation (Tb.Sp) $[\mu \mathrm{m}]$, and intraindividual distribution of separation (Tb.SpSD) [ $\mu \mathrm{m}]$ were determined to assess the trabecular compartment. ${ }^{11}$ From the cortical evaluation protocol, the cortical density (Ct.BMD) and the density of the cortical bone tissue (Ct.TMD) were obtained. The cortical thickness (Ct.Th) [ $\mu \mathrm{m}]$, cortical porosity (Ct.Po) [\%], and cortical pore diameter (Ct.Po.Dm) $[\mu \mathrm{m}]$ were determined to assess the cortical compartment. ${ }^{32}$

\section{Cortical interruption detection algorithm}

The algorithm is developed within the scanner software (Image Processing Language (IPL)). The first part of the algorithm has been described in detail elsewhere. ${ }^{26,27}$ In short, first, a cortical mask with a constant depth of 4 voxels $(0.328 \mathrm{~mm})$ is generated in 3D based on the outer contour. Second, the cortical bone within the masked region is analyzed for discontinuities that can be considered cortical interruptions (diameter $\geq 0.41 \mathrm{~mm}$ ), because these were also detected on $\mu \mathrm{CT}$ scans. ${ }^{27}$

The extended part of the algorithm is explained in Figure 5.2. As an example, two cortical interruptions $\geq 0.41 \mathrm{~mm}$ are visualized on a $2 \mathrm{D}$ grayscale image as output of the first part of the algorithm (Figure 5.2a, green circles). A region of interest (ROI) is obtained by dilating the detected cortical interruptions 48 times $(=3.936 \mathrm{~mm})$ in all three dimensions, which results in a sphere with a radius of $4 \mathrm{~mm}$. This contour was masked with the outer contour to only consider the region within the bone (Figure 5.2b). A radius of $4 \mathrm{~mm}$ was chosen because it approximates half the width of the metacarpal head (Figure 5.2b). The $\mathrm{ROI}$ prevents connection of detected voids with the intramedullary canal void. Within this ROI, a 3D distance transformation is performed, and only trabecular voids that are $\geq 0.738 \mathrm{~mm}$ in diameter are selected (Figure 5.2c), which is higher than trabecular separation commonly $(<5 \%)$ observed in MCP joints of healthy controls. ${ }^{13}$

The detected voids are then eroded by 2 voxels in all three dimensions, which leads to loss of connections of $\leq 0.328 \mathrm{~mm}$ in diameter, and therefore prevents leaking of the voids into the trabecular structure (Figure 5.2d). Only those voids that remain connected to a cortical interruption are included (Figure 5.2e). Last, these selected voids are dilated 
in all three dimensions to their original size and the originally detected cortical interruptions are added to these voids (Figure 5.2f).

Per joint, the number of cortical interruptions detected, interruption surface area, and interruption volume (cortical interruption + trabecular void volume) were obtained.
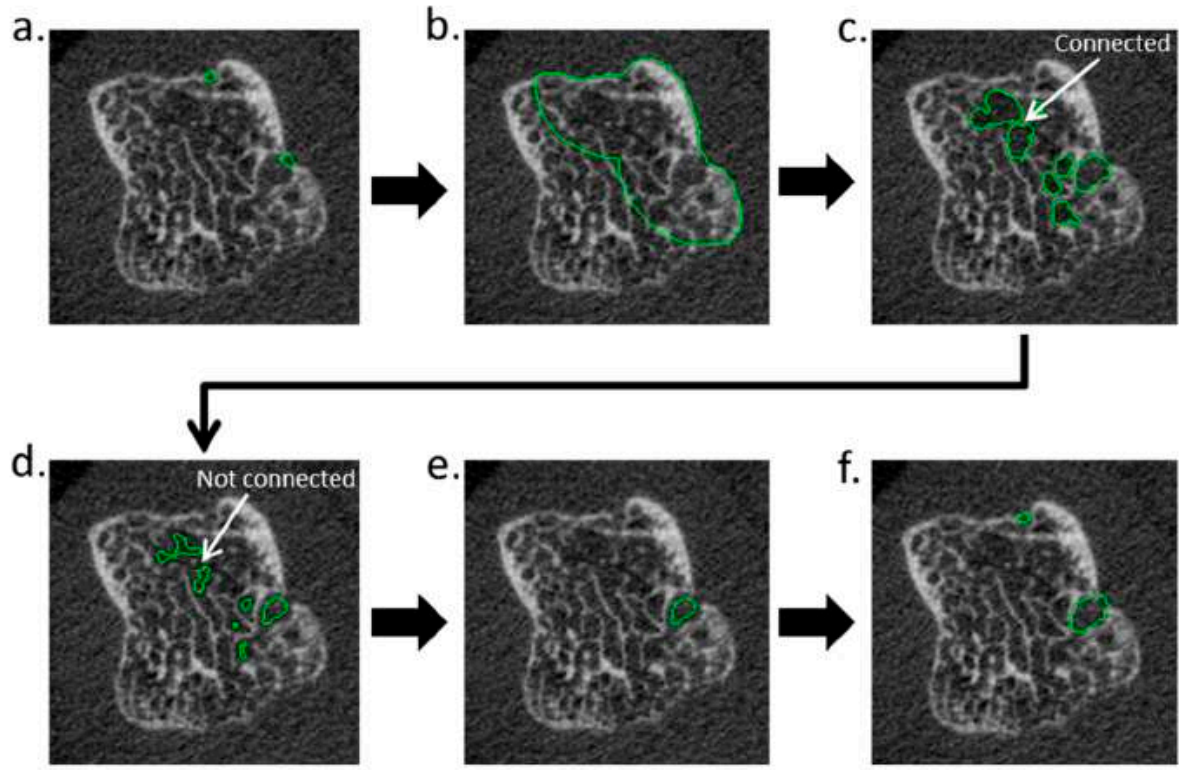

Figure 5.2. Representation of the steps executed by the extended part of the algorithm. (a.) Two cortical interruptions $\geq 0.41 \mathrm{~mm}$ are visualized on a $2 \mathrm{D}$ grayscale image as output of the first part of the algorithm. A region of interest $(\mathrm{ROI})$ is obtained by dilating the detected cortical interruptions with 48 voxels $(=3.936 \mathrm{~mm})$ and masked with the outer contour (b.). Within this ROI, a distance transformation is performed. Only voids that are $\geq 0.738 \mathrm{~mm}$ in diameter are selected (c.). These volumes are eroded by 2 voxels to lose connections of $\leq 0.328 \mathrm{~mm}$ and therefore prevent leaking into the trabecular structure (d.). The voids that remain connected to a cortical interruption are included (e.), dilated to its original size and the originally detected cortical interruptions are added (f.).

\section{Statistical analysis}

Descriptive statistics per joint were calculated for the number of interruptions, interruption surface area and volume, and bone density and micro-structure parameters. Paired t-test was used to compare the results per joint between the first and second scan assessments. Intra- and inter-operator reliability and intra-operator reproducibility on the joint level was assessed by intra-class correlation coefficient (ICC) with a two-way random model and absolute agreement. ICCs were rated as: $<0.40$ poor, 0.40-0.60 fair, 0.60-0.75 good, and 0.75-1.00 excellent. In addition, intra-operator reliability and reproducibility errors were determined as the root mean square (RMS) of coefficients of variation (CV) and the RMS of the standard deviation (SD), respectively 
$\mathrm{CV}_{\text {RMS }}$ and $\mathrm{SD}_{\mathrm{RMS}}$, as described by Glüer et al.. ${ }^{34}$ The Least Significant Change (LSC) was calculated in absolute values ( LSC $_{\mathrm{SD}}$ ) and in percentages ( $\mathrm{LSC}_{\mathrm{CV} \%}$ ) according to equation 1 and 2. Bland-Altman plots were made for qualitative assessment of the intra- and interoperator reliability, and intra-operator reproducibility for all parameters. Statistical analyses were performed using IBM SPSS Statistics for Windows, Version 20.0 (IBM Corp., Armonk, NY).

$$
\begin{array}{ll}
\mathrm{LSC}_{\mathrm{SD}}=1.96 \times \sqrt{ } 2 \times \mathrm{SD}_{\mathrm{RMS}} & \text { Eq. } 1 \\
\mathrm{LSC}_{\mathrm{CV} \%}=1.96 \times \sqrt{ } 2 \times \mathrm{CV}_{\mathrm{RMS}} & \text { Eq. } 2
\end{array}
$$

\section{RESULTS}

In 42 different patient scans with 126 stacks, 20 (15.9\%) stacks of poor quality were observed at the first scan and $13(10.3 \%)$ at the second scan. Two stacks were of poor quality at both the first and second scan. Therefore, in total 31 out of 126 stacks (24.6\%) were excluded from the analysis due to motion artifacts. Two joints, which had poor quality on all three stacks, were excluded (Figure 5.3). Hence, 40 joints remained for analysis (Figure 5.3).

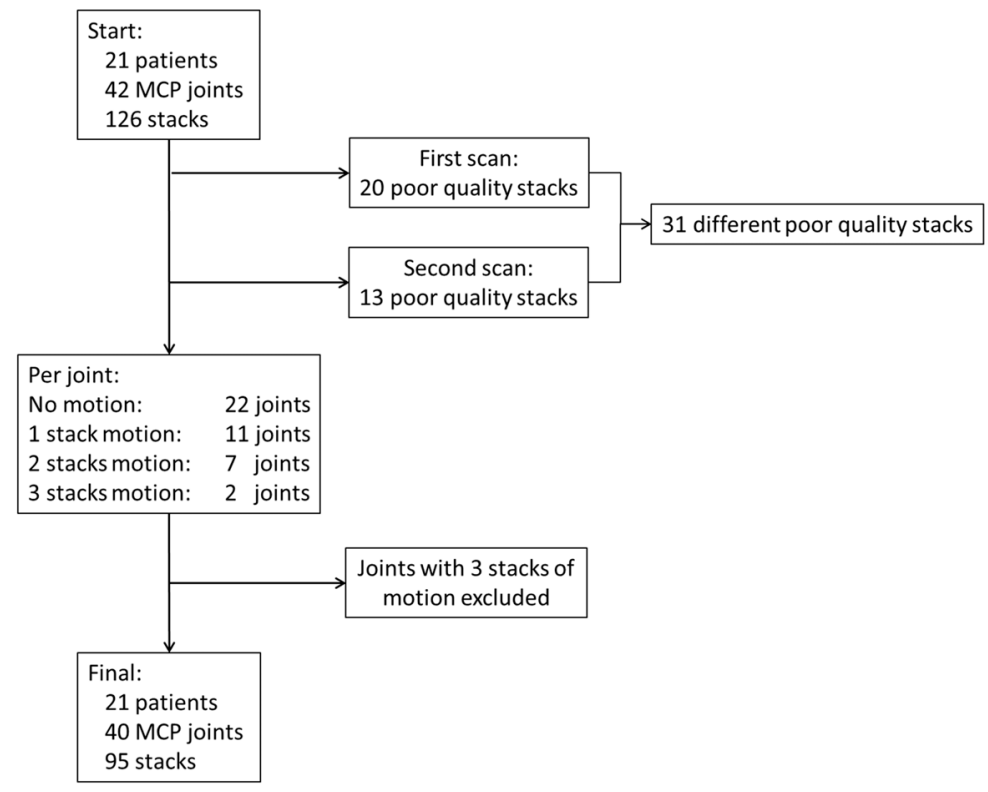

Figure 5.3. Schematic overview of the exclusion of joints due to motion artifacts. 


\section{Visual assessment}

Cortical interruptions detected by the algorithm based on the corrected contours of OP1 and OP2 on the first scan and corrected contours of OP1 on the second scan are visualized in 3D, and visualized on corresponding 2D grayscale images (Figure 5.4). Figure $5.4 a$ shows that the algorithm accurately detects cortical interruptions (green) and its underlying trabecular void volume (red), and that most interruptions were detected on both the first and second scan (green arrows). However, discrepancies were also found (red arrow).

Similarly, most interruptions detected by the algorithm on the first scan using the corrected contours of OP1 were also found when using the corrected contours of OP2 (green arrows), but discrepancies were found as well (red arrow).

OP1

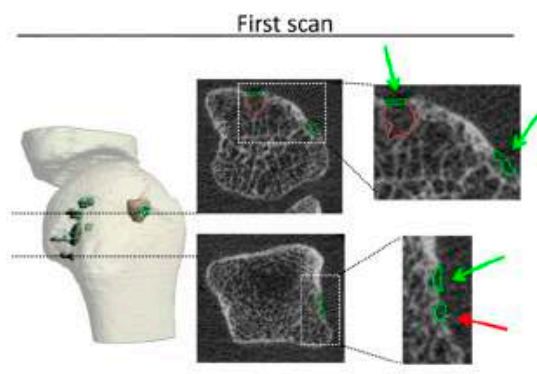

b.

OP2

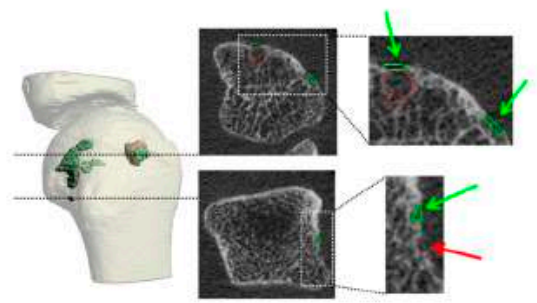

Second scan

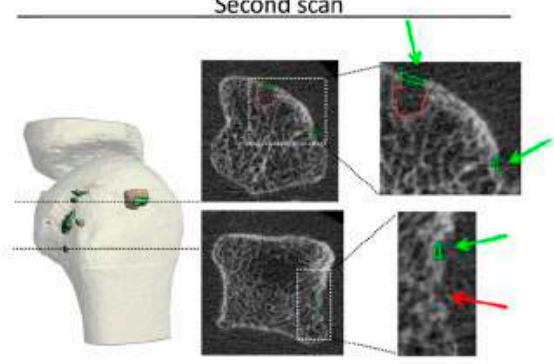

Figure 5.4. Visual outputs of the algorithm in 3D (I) and 2D (II) of an MCP joint. The outputs of OP1 (upper) and OP2 (lower) at the first (a.) and second scan performed (b.) are shown. (I) Shows the 3D outputs and multiple cortical interruptions (green) and underlying trabecular bone voids (red) are detected. (II) Shows the corresponding 2D grayscale images, and that the algorithm indeed accurately fills the underlying trabecular bone voids. It can be seen that most interruptions are detected at the same location on the first (a.) and second (b.) scan, and by both OP1 and OP2 (green arrows). However, some discrepancies were also seen (red arrows). 
$76 \mid$ Chapter 5

\section{Quantitative assessment}

Reliability

Intra- and inter-operator reliability was excellent for all bone density and micro-structure parameters (ICC >0.99). Bland-Altman plots show that no cut-off bias was observed for all bone density and micro-structure parameters, and that the errors were independent of the mean values detected (Supplementary Figures S5.2-S5.3). Intra- and interoperator reliability of the cortical interruption algorithm was excellent for the cortical interruption parameters (ICCs $\geq 0.91$ ), except for the inter-operator reliability of the interruption surface area (ICC $=0.70$, Supplementary Table S5.1). For the intra-operator reliability, LSC $_{S D}$ values were 2.0 for the number of interruptions, $4.6 \mathrm{~mm}^{2}$ for the interruption surface area, and $1.9 \mathrm{~mm}^{3}$ for the interruption volume (Supplementary Table S5.1). Bland-Altman plots show no cut-off bias and the errors were independent of the mean values detected (Supplementary Figure S5.5a). For the inter-operator reliability, Bland-Altman plots show no cut-off bias for the number of interruptions and the errors were independent of the number of interruptions detected (Supplementary Figure S5.5b). However, for the interruption surface area and volume, OP2 had higher outcomes compared to OP1, and this increased with increasing mean value (Supplementary Figure S5.5b).

\section{Reproducibility}

Table 5.1 shows the results for the bone density and micro-structure parameters, and the number of interruptions, interruption surface area and interruption volume detected by OP1 on the first and second scan. No statistical difference was found for all outcomes between the first and second scan.

The reproducibility for the bone density and micro-structure parameters was excellent with ICCS $\geq 0.84$ (Table 5.1). The precision errors in percentages $\left(\mathrm{CV}_{\mathrm{RMS}}\right)$ were generally $<5 \%$, except for Tb.SpSD, Ct.Po and Ct.Po.Dm $\left(\mathrm{CV}_{\mathrm{RMS}}=7.7 \%, 8.7 \%\right.$ and $7.6 \%$, respectively). The $\mathrm{LSC}_{\mathrm{CV} \%}$ was $\leq 7.3 \%$ for the bone density parameters, but up to $24.1 \%$ for the bone micro-structure parameters. Bland-Altman plots show that for the intraoperator reproducibility no cut-off bias was observed for all bone density and microstructure parameters, and that the errors were independent of the mean values detected (Supplementary Figure S5.4).

Reproducibility of the cortical interruption algorithm was also excellent for all outcomes with ICCs $\geq 0.82$ (Table 5.1), especially for the interruption volume (ICC 0.99, Table 5.1). The precision errors $S_{\text {RMS }}$ per joint was 1.5 for the number of interruptions, $2.1 \mathrm{~mm}^{2}$ for the interruption surface area, and $1.1 \mathrm{~mm}^{3}$ for the interruption volume. The $\mathrm{LSC}_{\mathrm{SD}}$ was 4.2 for the number of interruptions, $5.8 \mathrm{~mm}^{2}$ for interruption surface area, and $3.2 \mathrm{~mm}^{3}$ for the interruption volume. Bland-Altman plots show that for the intra-operator reproducibility no cut-off bias was observed for the number, surface area and volume of 
interruptions, and that the errors were independent of the mean values detected (Supplementary Figure S5.5c).

Table 5.1. Reproducibility of the cortical interruption parameters, and bone density and structure parameters.

\begin{tabular}{|c|c|c|c|c|c|c|}
\hline & Mean (SD) & $\operatorname{ICC}(95 \% \mathrm{CI})$ & $\mathrm{SD}_{\mathrm{RMS}}$ & $\mathrm{LSC}_{\mathrm{SD}}$ & $\mathrm{CV}_{\mathrm{RMS}}$ & $\mathrm{LSC}_{\mathrm{CV} \%}$ \\
\hline \multicolumn{7}{|l|}{ Cortical interruption parameters } \\
\hline Number of interruptions & $3.1(3.4)$ & $0.82(0.69-0.90)$ & 1.5 & 4.2 & n.a. & n.a. \\
\hline Interruption surface area $\left(\mathrm{mm}^{2}\right)$ & $4.2(7.1)$ & $0.92(0.85-0.96)$ & 2.1 & 5.8 & n.a. & n.a. \\
\hline Interruption volume $\mathrm{mm}^{3}$ & $3.5(10.7)$ & $0.99(0.98-0.99)$ & 1.1 & 3.2 & n.a. & n.a. \\
\hline \multicolumn{7}{|l|}{ Bone density parameters } \\
\hline Tot.BMD (mg HA/cm³) & $341.8(72.2)$ & $1.00(1.00-1.00)$ & 3.8 & 10.6 & 1.1 & 3.2 \\
\hline Tb.BMD (mg HA/ $\left.\mathrm{cm}^{3}\right)$ & $163.4(47.2)$ & $0.99(0.99-1.00)$ & 3.6 & 10.0 & 2.6 & 7.3 \\
\hline Ct.BMD (mg HA/ $\mathrm{cm}^{3}$ ) & 883.7 (94.5) & $1.00(1.00-1.00)$ & 4.5 & 12.4 & 0.5 & 1.4 \\
\hline Ct.TMD (mg HA/ $\left./ \mathrm{cm}^{3}\right)$ & $939.8(70.6)$ & $1.00(1.00-1.00)$ & 3.3 & 9.1 & 0.4 & 1.0 \\
\hline \multicolumn{7}{|l|}{ Bone structure parameters } \\
\hline Tb.N $\left(\mathrm{mm}^{-1}\right)$ & $1.56(0.49)$ & $0.99(0.98-0.99)$ & 0.06 & 0.16 & 4.2 & 11.7 \\
\hline Tb.Th $(\mu \mathrm{m})$ & $90.7(22.7)$ & $0.98(0.95-0.99)$ & 3.6 & 10.1 & 4.3 & 11.8 \\
\hline Tb.Sp $(\mu \mathrm{m})$ & $630.4(259.5)$ & $0.98(0.96-0.99)$ & 39.6 & 109.7 & 4.4 & 12.1 \\
\hline Tb.SpSD $(\mu \mathrm{m})$ & $558.3(316.2)$ & 0.97 (0.95-0.99) & 54.0 & 149.7 & 7.7 & 21.3 \\
\hline Ct.Th $(\mu \mathrm{m})$ & $889.7(196.2)$ & 1.00 (0.99-1.00) & 15.1 & 41.8 & 1.4 & 3.9 \\
\hline Ct.Po $(\%)$ & $4.31(3.07)$ & 1.00 (0.99-1.00) & 0.35 & 0.96 & 8.7 & 24.1 \\
\hline Ct.Po.Dm $(\mu \mathrm{m})$ & $197.3(86.3)$ & $0.84(0.72-0.91)$ & 36.7 & 101.7 & 7.6 & 21.1 \\
\hline
\end{tabular}

Values are displayed as mean (SD), and for ICC as value (95\% Confidence Interval). n.a., not applicable; $\mathrm{CV}_{\text {RMS }}$ and $\mathrm{LSC}_{\mathrm{CV} \%}$ were only determined for continuous variables. ICC, intra-class correlation coefficient; $\mathrm{SD}_{\mathrm{RMS}}$, root mean square of the standard deviation; $\mathrm{LSC}_{\mathrm{SD}}$, absolute least significant change; $\mathrm{CV}_{\mathrm{RMS}}$ root mean square of the coefficient of variation; $\mathrm{LSC}_{\mathrm{CV} \%}$, least significant change in percentages; Tot.BMD, total volumetric bone mineral density; Tb.BMD, trabecular BMD; Ct.BMD, cortical BMD; Ct.TMD, cortical bone tissue BMD; Tb.N, trabecular number; Tb.Th, trabecular thickness; Tb.Sp, trabecular separation; Tb.SpSD, intra-individual distribution of trabecular separation; Ct.Th, cortical thickness; Ct.Po, cortical porosity; Ct.Po.Dm, cortical porosity diameter

\section{DISCUSSION}

In this study, we calculated the precision errors of our extended algorithm for detection of cortical interruptions and underlying trabecular bone void volume in MCP joints on scan/re-scan HR-pQCT data with repositioning in-between the scans in early arthritis patients. In addition, we calculated the precision errors for the bone density and microstructure parameters. Reproducibility of our algorithm was excellent (ICCs $\geq 0.82$ ), especially for the interruption volume (ICC 0.99). Reproducibility for the bone density and micro-structure parameters was also excellent (ICCs $\geq 0.84$ ). Bland-Altman plots showed no systematic error in the reproducibility of our algorithm, bone density and bone micro-structure parameters. The reproducibility LSC $_{S D}$ value per joint was 4.2 for number of interruptions, $5.8 \mathrm{~mm}^{2}$ for interruption surface area, and $3.2 \mathrm{~mm}^{3}$ for interruption volume.

The intra-operator reproducibility LSC $_{S D}$ value of the algorithm for the interruption volume was higher in our study than the intra- and inter-operator LSC $_{S D}$ reported by Töpfer et al. for single interruptions (LSC $3.2 \mathrm{~mm}^{3}$ versus $1.4 \mathrm{~mm}^{3}$ and $2.1 \mathrm{~mm}^{3}$, 
respectively). ${ }^{21}$ The study from Töpfer et al. differed in several aspects from ours. They analyzed a selection of interruptions in one dataset on its volume by two operators. In contrast, we used scan/re-scan data and included all interruptions, irrespective whether they were detected on the first scan but not on the second scan and vice versa. These aspects will lead to higher reproducibility errors. By excluding the effect of the rescanning (i.e. intra-operator reliability), the $\mathrm{LSC}_{\mathrm{SD}}$ value was comparable to the study of Töpfer et al. (LSC $1.9 \mathrm{~mm}^{3}$ versus $1.4 \mathrm{~mm}^{3}$ ). ${ }^{21}$

In our study, we also included the phalangeal base, thus creating a larger scan region for analyzing bone density and micro-structure parameters. This did not affect the precision errors, except for Ct.Po, which was substantially lower compared to a previous study (8.7\% versus $27.7 \%) .{ }^{28}$ The precision errors of the other parameters obtained in our study were similar as in previous studies. ${ }^{13,28}$ In our study, the precision errors ( $\left.\mathrm{CV}_{\text {RMS }}\right)$ were generally below $2 \%$ for the bone density parameters (except for Tb.BMD), below $5 \%$ for the trabecular bone parameters (except for Tb.SpSD), below 10\% for the cortical bone parameters. The precision errors are also comparable as observed in radius and tibia scans. $^{11}$

The mean number of cortical interruptions and interruption surface area per joint detected in this study (3.1 and $4.2 \mathrm{~mm}^{2}$, respectively) were substantially lower than in our previously reported study using the same algorithm (9.5 and $13.5 \mathrm{~mm}^{2}$, respectively). ${ }^{27}$ In our previous study, anatomic specimens from high-aged subjects (mean 85.1 years) were used with a low BMD (vBMD of the joints: $245 \mathrm{mgHA} / \mathrm{cm}^{3}$ versus $338 \mathrm{mgHA} / \mathrm{cm}^{3}$ in this study). In the present study, the bone is better mineralized and therefore these voxels are less likely to represent non-bone voxels after segmentation and, hence, a lower number of interruptions was found.

The mean volume of the interruptions detected with the algorithm was substantially lower compared to previous studies that investigated volumes of interruptions in 3D $\left(1.1 \mathrm{~mm}^{3}\right.$ versus $\left.>4 \mathrm{~mm}^{3}\right)$, confirming that our algorithm enables the detection of much smaller interruptions. ${ }^{13,21,24}$

Our study has several limitations. First, with our algorithm, the trabecular void volume underlying the cortical interruption that can be detected is limited to a depth of $4 \mathrm{~mm}$. This means that the algorithm underestimates the volume of interruptions with a depth greater than $4 \mathrm{~mm}$. However, $4 \mathrm{~mm}$ is approximately half the width of the metacarpal head. Hence, such interruptions are not the primary focus of research with HR-pQCT, because these large interruptions can also be detected by other imaging techniques with lower resolution. For example, "small" interruptions, i.e. $<10 \mathrm{~mm}^{3}$ are occasionally missed with MRI. ${ }^{12}$ Thus, our algorithm can best be used for studies with HR-pQCT aiming at early detection of structural damage, i.e. small interruptions, in patients with RA. Second, our algorithm requires manual correction of the outer margin of the contours in case of large cortical interruptions and motion artifacts which can make the analysis time consuming. ${ }^{26}$ However, this correction is also advised by the manufacturer for the standard evaluation protocol for assessment of the bone density and microstructure parameters. Further automation of the outer contour would improve the 
applicability. The strength of our algorithm is that it is developed within the scanner software (IPL). Therefore, the algorithm can be easily implemented to other scanners.

The current investigation of the reproducibility of the algorithm and the extension of underlying trabecular bone void detection was a next step in the validation of our algorithm in the detection of small cortical interruptions in finger joints by HR-pQCT. We found that the algorithm was highly reproducible, but still had substantial precision errors compared to the mean value detected. Therefore, the next step is to test this algorithm in clinical studies in order to determine its potential value in monitoring patients with RA, and discriminating patients with RA, preferably early in the disease course, from healthy controls.

\section{Conclusion}

The extended algorithm for detection of cortical interruptions and their volume, and the assessment of the bone density and micro-structure parameters on HR-pQCT is highly reproducible in finger joints of early arthritis patients. The potential value of this algorithm for monitoring structural damage in the joints in early arthritis patients needs to be tested in clinical studies. 


\section{REFERENCES}

1. Schett G, Gravallese E. Bone erosion in rheumatoid arthritis: mechanisms, diagnosis and treatment. Nature reviews Rheumatology. 2012;8(11):656-64.

2. Geusens P, van den Bergh J. Bone erosions in rheumatoid arthritis. Rheumatology. 2014;53(1):4-5.

3. Baillet A, Gaujoux-Viala C, Mouterde G, Pham T, Tebib J, Saraux A, et al. Comparison of the efficacy of sonography, magnetic resonance imaging and conventional radiography for the detection of bone erosions in rheumatoid arthritis patients: a systematic review and meta-analysis. Rheumatology. 2011;50(6):1137-47.

4. Dohn UM, Ejbjerg BJ, Hasselquist M, Narvestad E, Moller J, Thomsen HS, et al. Detection of bone erosions in rheumatoid arthritis wrist joints with magnetic resonance imaging, computed tomography and radiography. Arthritis Res Ther. 2008;10(1):R25.

5. Geusens P, Chapurlat R, Schett G, Ghasem-Zadeh A, Seeman E, de Jong J, et al. High-resolution in vivo imaging of bone and joints: a window to microarchitecture. Nature reviews Rheumatology. 2014;10(5):304-13.

6. Ejbjerg B, Narvestad E, Rostrup E, Szkudlarek M, Jacobsen S, Thomsen HS, et al. Magnetic resonance imaging of wrist and finger joints in healthy subjects occasionally shows changes resembling erosions and synovitis as seen in rheumatoid arthritis. Arthritis Rheum. 2004;50(4):1097-106.

7. Boutroy S, Bouxsein ML, Munoz F, Delmas PD. In vivo assessment of trabecular bone microarchitecture by high-resolution peripheral quantitative computed tomography. J Clin Endocrinol Metab. 2005;90(12):6508-15.

8. Stach CM, Bauerle M, Englbrecht M, Kronke G, Engelke K, Manger B, et al. Periarticular bone structure in rheumatoid arthritis patients and healthy individuals assessed by high-resolution computed tomography. Arthritis Rheum. 2010;62(2):330-9.

9. Srikhum W, Virayavanich W, Burghardt AJ, Yu A, Link TM, Imboden JB, et al. Quantitative and semiquantitative bone erosion assessment on high-resolution peripheral quantitative computed tomography in rheumatoid arthritis. J Rheumatol. 2013;40(4):408-16.

10. Albrecht A, Finzel S, Englbrecht M, Rech J, Hueber A, Schlechtweg $P$, et al. The structural basis of MRI bone erosions: an assessment by microCT. Ann Rheum Dis. 2013;72(8):1351-7.

11. Finzel S, Ohrndorf S, Englbrecht M, Stach C, Messerschmidt J, Schett G, et al. A detailed comparative study of high-resolution ultrasound and micro-computed tomography for detection of arthritic bone erosions. Arthritis Rheum. 2011;63(5):1231-6.

12. Scharmga A, Peters M, van Tubergen A, van den Bergh J, de Jong J, Loeffen D, et al. Visual detection of cortical breaks in hand joints: reliability and validity of high-resolution peripheral quantitative $C T$ compared to microCT. BMC Musculoskelet Disord. 2016;17(1):271.

13. Fouque-Aubert A, Boutroy S, Marotte H, Vilayphiou N, Bacchetta J, Miossec P, et al. Assessment of hand bone loss in rheumatoid arthritis by high-resolution peripheral quantitative CT. Ann Rheum Dis. 2010;69(9):1671-6.

14. Krause M, Museyko O, Breer S, Wulff B, Duckstein C, Vettorazzi E, et al. Accuracy of trabecular structure by HR-pQCT compared to gold standard muCT in the radius and tibia of patients with osteoporosis and long-term bisphosphonate therapy. Osteoporos Int. 2014;25(5):1595-606.

15. Peters M, de Jong J, Scharmga A, van Tubergen A, Loeffen D, Weijers R, et al. The reproducibility of a semi-automatic algorithm in the detection of cortical breaks and adjacent trabecular bone loss in scanrescan data from patients with early arthritis [abstract]. Arthritis Rheum. 2016;68 (suppl 10).

16. Burghardt AJ, Buie HR, Laib A, Majumdar S, Boyd SK. Reproducibility of direct quantitative measures of cortical bone microarchitecture of the distal radius and tibia by HR-pQCT. Bone. 2010;47(3):519-28.

17. Buie HR, Campbell GM, Klinck RJ, MacNeil JA, Boyd SK. Automatic segmentation of cortical and trabecular compartments based on a dual threshold technique for in vivo micro-CT bone analysis. Bone. 2007;41(4):505-15.

18. Laib A, Hauselmann HJ, Ruegsegger P. In vivo high resolution 3D-QCT of the human forearm. Technol Health Care. 1998;6(5-6):329-37.

19. Tjong W, Kazakia GJ, Burghardt AJ, Majumdar S. The effect of voxel size on high-resolution peripheral computed tomography measurements of trabecular and cortical bone microstructure. Med Phys. 2012; 39(4):1893-903. 
20. Tjong W, Nirody J, Burghardt AJ, Carballido-Gamio J, Kazakia GJ. Structural analysis of cortical porosity applied to HR-pQCT data. Med Phys. 2014;41(1):013701.

21. Zebaze RM, Ghasem-Zadeh A, Bohte A, Iuliano-Burns S, Mirams M, Price RI, et al. Intracortical remodelling and porosity in the distal radius and post-mortem femurs of women: a cross-sectional study. Lancet. 2010;375(9727):1729-36.

22. Finzel S, Rech J, Schmidt S, Engelke K, Englbrecht M, Schett G. Interleukin-6 receptor blockade induces limited repair of bone erosions in rheumatoid arthritis: a micro CT study. Ann Rheum Dis. 2013;72(3): 396-400.

23. Finzel S, Rech J, Schmidt S, Engelke K, Englbrecht M, Stach C, et al. Repair of bone erosions in rheumatoid arthritis treated with tumour necrosis factor inhibitors is based on bone apposition at the base of the erosion. Ann Rheum Dis. 2011;70(9):1587-93. 


\section{SUPPLEMENTARY FILE}

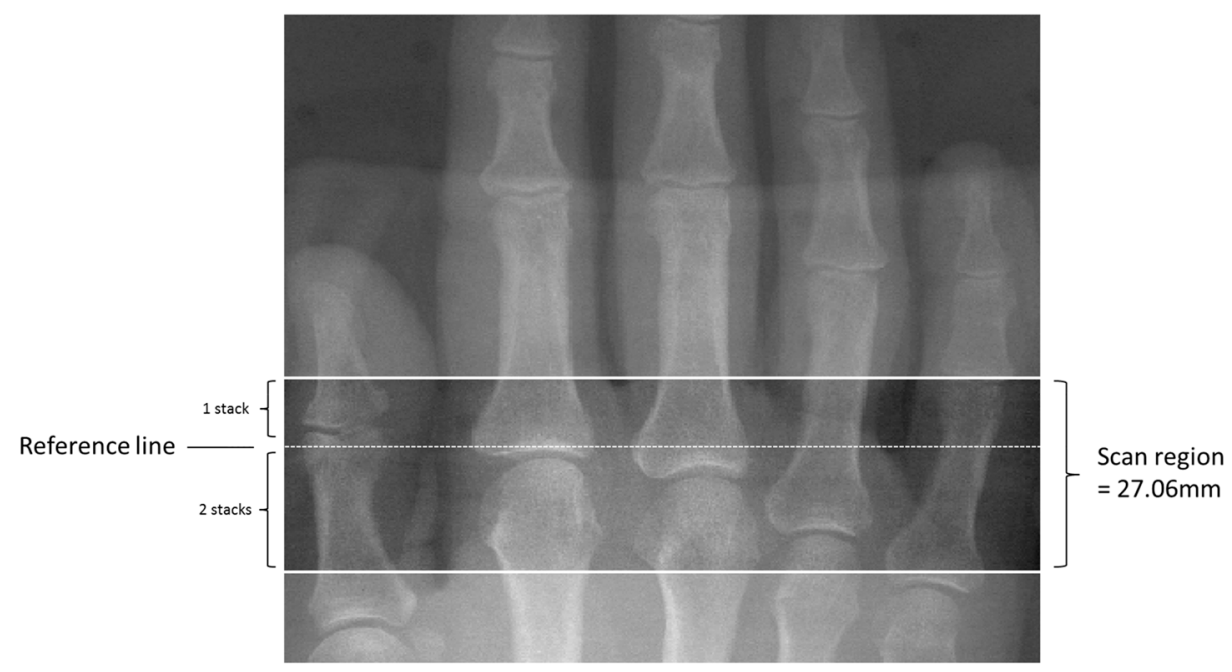

Figure S5.1. Scout view of the right hand, showing the region that was scanned by the HR-pQCT during both scans. The proximal edge of the phalangeal base of the most distal joint (MCP2 in this case) was chosen as the landmark for the placement of the reference line. The scan region was $27.06 \mathrm{~mm}$ (3 stacks) long with $9.02 \mathrm{~mm}$ (1 stack) distal of the reference line and $18.04 \mathrm{~mm}$ (2 stacks) proximal of the reference line. 
Tot.BMD

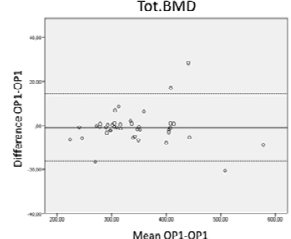

Tb. N

B

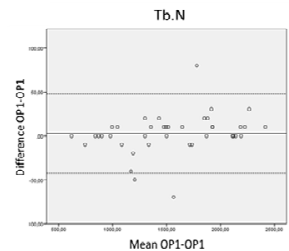

Ct.Th

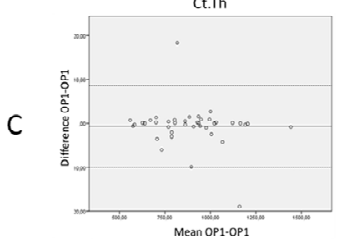

Tb.BMD

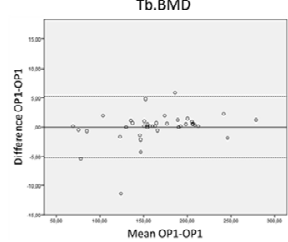

Tb.Th

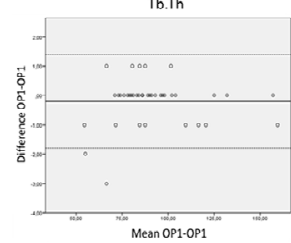

Ct.Po

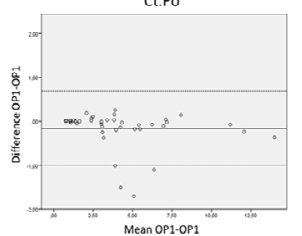

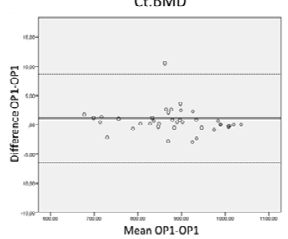

Tb.Sp

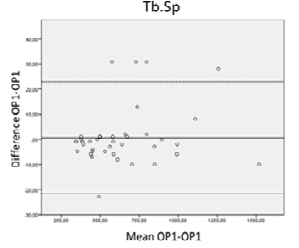

Ct.Po.Dm

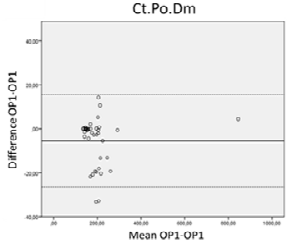

Ct.TMD

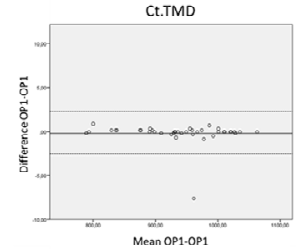

Th. $5 p 50$

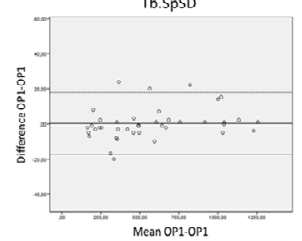

Mean OP1-OP1

Figure S5.2. Bland-Altman plots for all bone density (A), trabecular micro-structure (B) and cortical microstructure $(C)$ parameters for the intra-operator reliability. For all parameters, no cut-off bias was observed and the errors were independent of the mean values detected. BMD, volumetric bone mineral density; Tot.BMD, total BMD; Tb.BMD, trabecular BMD; Ct.BMD, cortical BMD; Ct.TMD, cortical bone tissue BMD; Tb.N, trabecular number; Tb.Th, trabecular thickness; Tb.Sp, trabecular separation; Tb.SpSD, intra-individual distribution of trabecular separation; Ct.Th, cortical thickness; Ct.Po, cortical porosity; Ct.Po.Dm, cortical porosity diameter. 
Tot.BMD

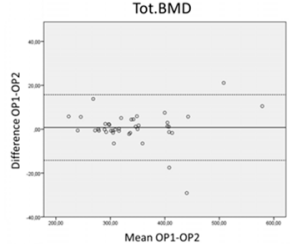

Tb.N

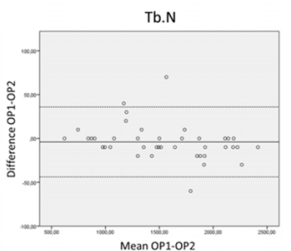

Ct.Th

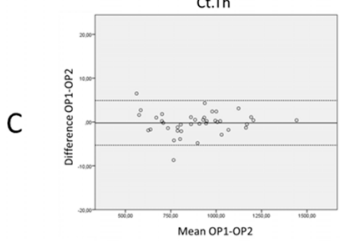

Tb.BMD

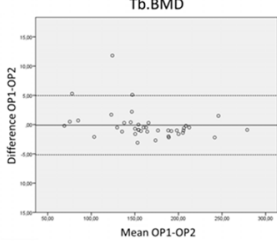

Tb.Th

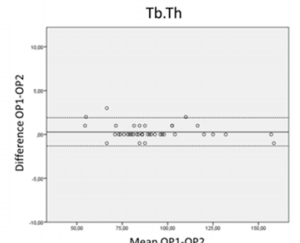

Ct.Po

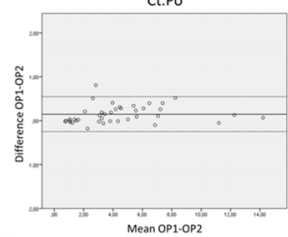

Ct.BMD

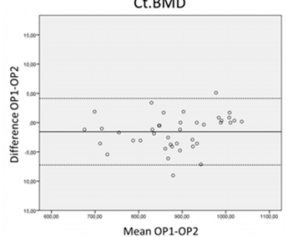

Tb.Sp

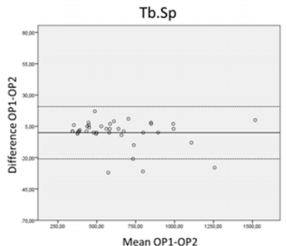

Ct.Po.Dm

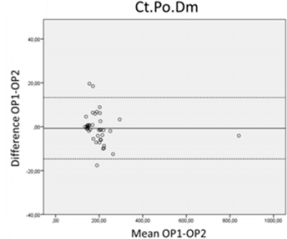

Ct.TMD

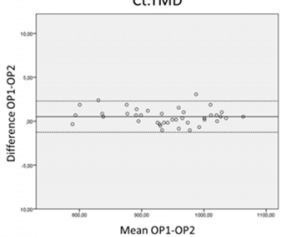

Tb.SpSD

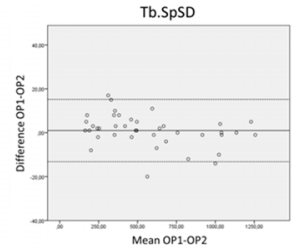

Figure S5.3. Bland-Altman plots for all bone density (A), trabecular micro-structure (B) and cortical microstructure $(C)$ parameters for the inter-operator reliability. For all parameters, no cut-off bias was observed and the errors were independent of the mean values detected. BMD, volumetric bone mineral density; Tot.BMD, total BMD; Tb.BMD, trabecular BMD; Ct.BMD, cortical BMD; Ct.TMD, cortical bone tissue BMD; Tb.N, trabecular number; Tb.Th, trabecular thickness; Tb.Sp, trabecular separation; Tb.SpSD, intra-individual distribution of trabecular separation; Ct.Th, cortical thickness; Ct.Po, cortical porosity; Ct.Po.Dm, cortical porosity diameter. 
Tot.BMD

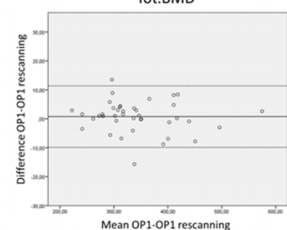

Tb.N

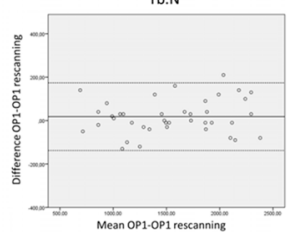

Ct.Th

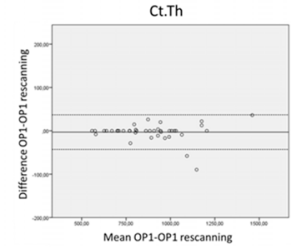

Tb.BMD

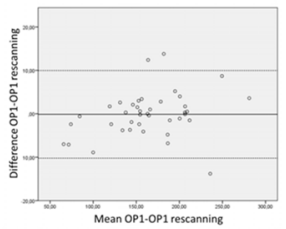

Tb.Th

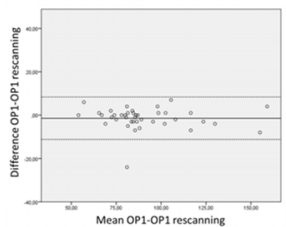

Ct.po

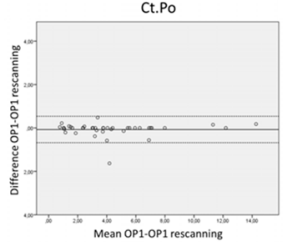

Ct.BMD

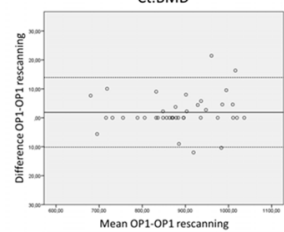

Tb.Sp

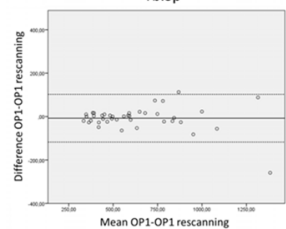

Ct.Po.Dm

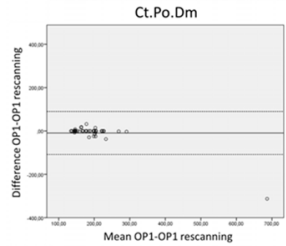

Ct.TMD

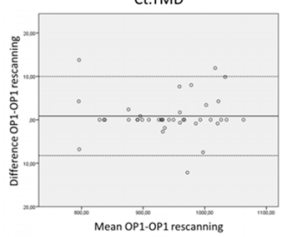

Tb.SpSD

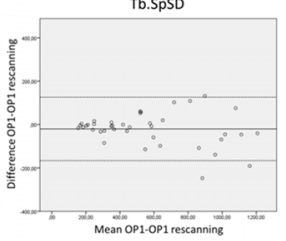

Mean OP1.OP1 rescanning

Figure S5.4. Bland-Altman plots for all bone density (A), trabecular micro-structure (B) and cortical microstructure (C) parameters for the intra-operator reproducibility. For all parameters, no cut-off bias was observed and the errors were independent of the mean values detected. $\mathrm{BMD}$, volumetric bone mineral density; Tot.BMD, total BMD; Tb.BMD, trabecular BMD; Ct.BMD, cortical BMD; Ct.TMD, cortical bone tissue BMD; Tb.N, trabecular number; Tb.Th, trabecular thickness; Tb.Sp, trabecular separation; Tb.SpSD, intraindividual distribution of trabecular separation; Ct.Th, cortical thickness; Ct.Po, cortical porosity; Ct.Po.Dm, cortical porosity diameter. 
A
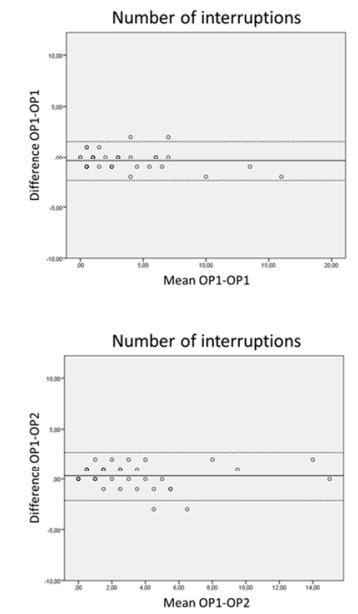

\section{B}

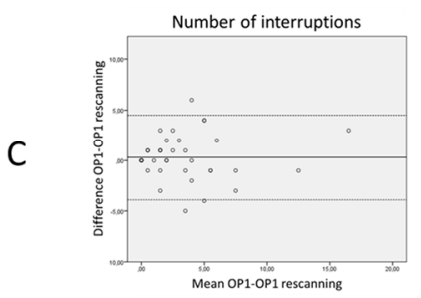

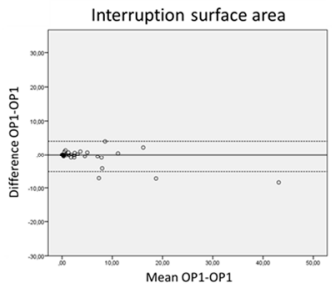
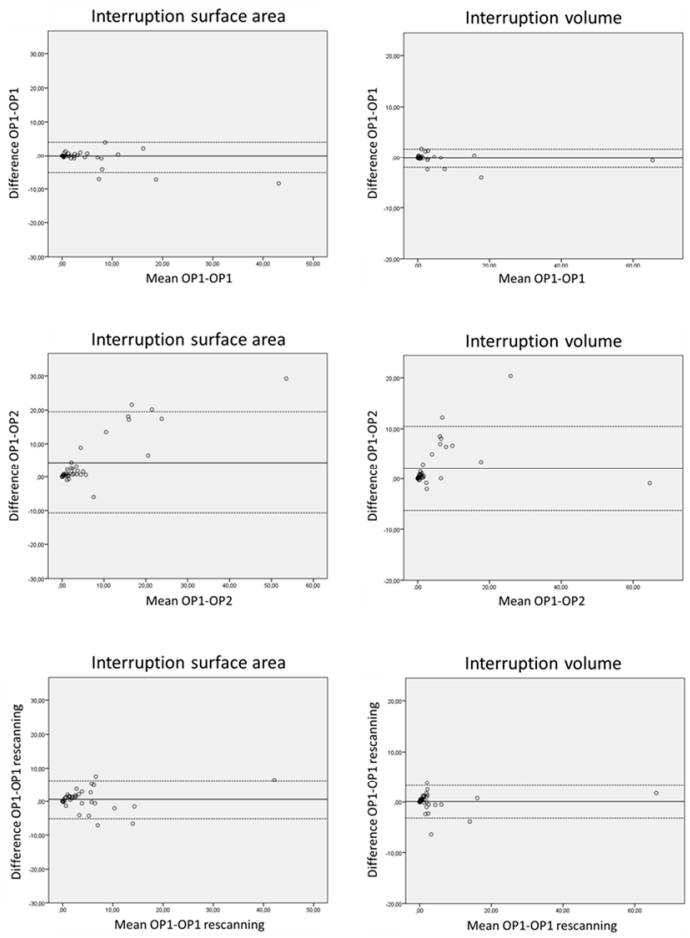

Figure S5.5. Bland-Altman plots for all cortical interruption parameters for the inter-operator reliability (A) and intra-operator reproducibility (B). (A) Between the operators, no cut-off bias was observed for the number of interruptions and the errors were independent of the number of interruptions detected. For the interruption surface area and volume, OP2 had higher outcomes compared to operator 1, and this increased with increasing mean value. (B) Between the first and second scanning, no cut-off bias was observed for the number, surface area and volume of interruptions and the errors were independent of the mean values detected.

Table S5.1. Intra- and inter-operator reliability of the cortical interruption parameters.

\begin{tabular}{|c|c|c|c|c|c|}
\hline & & \multicolumn{3}{|c|}{ Intra-operator reliability } & \multirow{2}{*}{$\begin{array}{c}\text { Inter-operator reliability } \\
\text { ICC } \\
(95 \% \mathrm{Cl}) \\
\end{array}$} \\
\hline & & $\begin{array}{c}\text { ICC } \\
(95 \% \mathrm{Cl}) \\
\end{array}$ & $\mathrm{SD}_{\mathrm{RMS}}$ & $\mathrm{LSC}_{\mathrm{SD}}$ & \\
\hline $\begin{array}{l}\text { Number of } \\
\text { interruptions }\end{array}$ & & $\begin{array}{c}0.96 \\
(0.92-0.98)\end{array}$ & 0.7 & 2.0 & $\begin{array}{c}0.94 \\
(0.89-0.97) \\
\end{array}$ \\
\hline $\begin{array}{l}\text { Interruption } \\
\text { surface area }\end{array}$ & $\mathrm{mm}^{2}$ & $\begin{array}{c}0.95 \\
(0.92-0.98)\end{array}$ & 1.7 & 4.6 & $\begin{array}{c}0.70 \\
(0.41-0.84)\end{array}$ \\
\hline $\begin{array}{l}\text { Interruption } \\
\text { volume }\end{array}$ & $\mathrm{mm}^{3}$ & $\begin{array}{c}1.00 \\
(0.99-1.00)\end{array}$ & 0.7 & 1.9 & $\begin{array}{c}0.91 \\
(0.81-0.96)\end{array}$ \\
\hline
\end{tabular}

Values are as value (95\% Confidence Interval). ICC, intra-class correlation coefficient; $\mathrm{SD}_{\mathrm{RMS}}$, root mean square of the standard deviation; LSC $_{S D}$, absolute least significant change. 


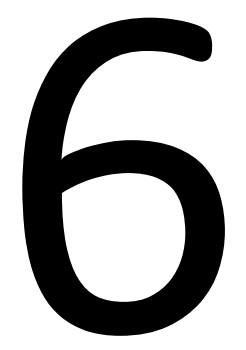

Assessment of cortical interruptions in the finger joints of patients with rheumatoid arthritis using HR-pQCT, radiography and MRI

M. Peters, A. van Tubergen, A. Scharmga, J.H.M. Driessen, B. van Rietbergen, D. Loeffen, R. Weijers, P. Geusens, J.P. van den Bergh 


\section{ABSTRACT}

\section{Objectives}

Small cortical interruptions may be the first sign of an erosion, and more interruptions can be found in patients with rheumatoid arthritis (RA) compared to healthy subjects. Therefore, we, first, compared the number and size of interruptions in patients with RA to healthy subjects using high-resolution peripheral quantitative CT (HR-pQCT). Second, we investigated the association between structural damage and inflammatory markers on conventional radiography (CR) and MRI with interruptions on HR-pQCT. Third, the added value of HR-pQCT over CR and MRI was investigated.

\section{Methods}

Finger joints of 39 patients with RA and 38 healthy subjects were examined through CR, MRI and HR-pQCT. CRs were scored using the Sharp/Van der Heijde method. MRI images were analyzed for the presence of erosions, bone marrow edema and synovitis. HRpQCT images were analyzed for the number, surface area and volume of cortical interruptions using a semi-automated algorithm. Descriptives were calculated, and associations were tested using generalized estimating equations.

\section{Results}

Significantly more interruptions and both a larger surface area and volume of interruptions were detected in MCP joints of patients with RA compared to HS (median 2.0, $1.42 \mathrm{~mm}^{2}$ and $0.48 \mathrm{~mm}^{3}$ versus $1.0,0.69 \mathrm{~mm}^{2}$ and $0.23 \mathrm{~mm}^{3}$, respectively, all $p<0.01$ ). Findings on $C R$ and $M R I$ were significantly associated with more and larger interruptions on HR-pQCT (prevalence ratios (PRs) 1.03-7.74, all $p<0.01$ ) in all subjects, and were consistent in patient with RA alone. Having RA is significantly associated with more and larger interruptions on HR-pQCT (PRs 2.33-5.39, all $p<0.01$ ), also after adjustment for findings on CR or MRI.

\section{Conclusions}

More and larger cortical interruptions and both a larger surface area and volume were found in finger joints of patients with RA compared to healthy subjects, also after adjustment for findings on CR or MRI, implying that HR-pQCT imaging may be of value in addition to $C R$ and MRI for the evaluation of structural damage in patients with RA. 
\begin{tabular}{c|l} 
Cortical interruptions in RA and healthy subjects & \\
89
\end{tabular}

\section{INTRODUCTION}

Rheumatoid arthritis (RA) is a chronic inflammatory auto-immune disease, in which inflammation at the joint might lead to peri-articular osteoporosis and erosions (i.e. pathological cortical interruptions). ${ }^{1,2}$ Traditionally, the presence of erosions is assessed by conventional radiography (CR) in the joints of the hands and feet. However, CR has its limitations since the sensitivity to detect erosions is low compared to computed tomography (CT) and magnetic resonance imaging (MRI). ${ }^{3-6}$ With $\mathrm{CT}$ as reference method, sensitivity to detect erosions in MCP joints was $26 \%$ and $68 \%$ for CR and MRI, respectively. ${ }^{7}$ In addition, $\mathrm{MRI}$ is also able to visualize inflammation in the joint such as bone marrow edema (BME) and synovitis, which are associated with progression of bone damage on CR in patients with RA. ${ }^{8-10}$

Another imaging modality used in RA is high-resolution peripheral quantitative $\mathrm{CT}$ (HR-pQCT), with which the three-dimensional (3D) bone structure of the peripheral skeleton can be visualized at the micro-scale ( $82 \mu \mathrm{m}$ isotropic voxel size) in vivo. Comparative studies showed that HR-pQCT imaging is more sensitive than MRI and CR in the detection of pathological interruptions (i.e. erosions) in finger joints. ${ }^{11-13}$ In addition, HR-pQCT imaging can evaluate the cortical and trabecular volumetric bone mineral density (VBMD) and bone micro-structure in finger joints. ${ }^{14}$

Previous studies that compared patients with RA to healthy subjects using HR-pQCT primarily focused on the visual detection of pathological interruptions. ${ }^{14-20}$ In a previous study, we demonstrated that with HR-pQCT a variety of cortical interruptions can be seen in finger joints of both patients with RA and healthy subjects. Some (larger) cortical interruptions had underlying trabecular bone voids and were suggestive for an erosion, whereas other cortical interruptions were smaller and may represent physiological interruptions (i.e. vascular channels). ${ }^{21}$ The detection of these smaller interruptions might be of clinical relevance, since it has been hypothesized that vascular channels might serve as a starting point for pathological cortical interruptions as the vascular channel provides direct entry for osteoclast-mediated joint destruction., ${ }^{1,22,23}$ It has also been shown that the number of visually detected small cortical interruptions is increased in patients with RA compared to healthy subjects. ${ }^{24}$ Moreover, the number of visually detected interruptions was increased in patients with a more active disease, suggesting a link to synovitis. ${ }^{11}$ Besides the detection of more and larger cortical interruptions in RA, also an impaired $V B M D$ and micro-structure can be found in finger joints of patients with RA compared to healthy subjects. ${ }^{14}$ These parameters at the MCP joints are strongly correlated to the parameters at the distal radius, which are impaired in osteoporosis patients. $^{25,26}$

A major drawback of visual detection of cortical interruptions is that it is not only prone to subjectivity but also time-consuming. ${ }^{27}$ In a previous study, we showed that the interoperator reliability of visually detecting these small cortical interruptions was fair. ${ }^{28}$ Therefore, we developed and validated a semi-automated algorithm that more reliably 
$90 \mid$ Chapter 6

detects the number, surface area and volume of (small) cortical interruptions (diameter $\geq 0.41 \mathrm{~mm}$ ) in comparison to visual scoring. ${ }^{28-31}$

Whereas these earlier studies demonstrated the feasibility, validity and reliability of our semi-automated algorithm in quantifying (small) cortical interruptions, it remains unknown if these (small) cortical interruptions are more frequently seen in patients with RA compared to controls, and whether they are related to both structural damage on CR and structural damage and inflammatory markers on MRI. Furthermore, it is unknown if HR-pQCT imaging has an added value over findings on CR and MRI in the detection of (small) cortical interruptions and in the distinction between patients with RA and healthy subjects.

Therefore, our aims were, first, to evaluate the number, surface area and volume of cortical interruptions (further described as "cortical interruption parameters") detected on HR-pQCT images with our semi-automated algorithm in patients with RA (dominant versus non-dominant hand, and early versus longstanding RA patients) and compare this to healthy subjects. Second, we investigated whether an association exists between structural damage on $\mathrm{CR}$, or structural and inflammatory markers on MRI, with the cortical interruption parameters on HR-pQCT in all subjects and in patients with RA alone. Third, we investigated the added value of HR-pQCT in the detection of cortical interruptions and in the distinction between patients with RA and healthy subjects over findings on $\mathrm{CR}$ and $\mathrm{MRI}$. Last, we investigated the bone density and micro-structure parameters in all subjects and in patients with RA alone, and their association with the cortical interruption parameters.

\section{MATERIALS AND METHODS}

\section{Subjects}

For this cross-sectional study, we used data from the MOSA-Hand cohort, which consists of 38 healthy females and 41 female patients diagnosed with RA. ${ }^{21}$ All subjects were recruited at the Maastricht University Medical Center, the Netherlands. All patients with RA fulfilled the 2010 American College of Rheumatology (ACR)/European League Against Rheumatism (EULAR) classification criteria for RA. ${ }^{32}$ Information on disease duration, medication use, smoking status, anti-citrullinated protein antibody (ACPA) and rheumatoid factor (RF) positivity, was retrieved from the patients' medical file. Healthy subjects, matched on age by decade, did not suffer from hand joint complaints. All subjects signed informed consent. Ethical approval was obtained from the ethics board of the academic hospital Maastricht/ Maastricht University, the Netherlands. Netherlands Trial Registry number: NTR3821. 
\begin{tabular}{c|l} 
& \\
Cortical interruptions in RA and healthy subjects & 91
\end{tabular}

\section{HR-pQCT image acquisition}

The second and third MCP and PIP joints were scanned with HR-pQCT (XtremeCT1, Scanco Medical AG, Switzerland). In healthy subjects only the dominant hand was scanned and in patients with RA both hands were scanned. Each hand was separately scanned using a carbon cast for finger scanning as provided by the manufacturer.

For the first 13 subjects ( 8 healthy subjects and 5 patients with RA) that were scanned, a scan protocol of 2 stacks was used for MCP joints and 1 stack for PIP joints (Figure 6.1a, Scan protocol 1). This scan length was in subsequent subjects enlarged to 3 stacks for MCP joints and 2 stacks for PIP joints, based on the acquired experience of the Study grouP for xtrEmeCT in RA (SPECTRA, Figure $6.1 b$, Scan protocol 2). ${ }^{33}$ With both scan protocols, the reference line of MCP scans was placed at the top of the second or third metacarpal head (whichever was most distal of the two), and for PIP scans at second or third proximal interphalange (whichever was most distal of the two). In this way, similar regions of interest were selected and measured. Figure 6.1 shows that both scan protocols encapsulate the whole joint region, i.e. including the total region where cortical interruptions may occur. Therefore, all scans were used for the quantification of cortical interruptions. However, for the bone density and micro-structure parameters, the scans of protocol 1 were excluded since a difference in scan length substantially influences these parameters (Figure 6.2).

Scanning was performed at clinical in vivo settings, i.e. at $60 \mathrm{kVp}$ tube voltage, $900 \mu \mathrm{A}$ tube current, 100ms integration time and images were reconstructed using an $82 \mu \mathrm{m}$ isotropic voxel size. Subject scans were evaluated on motion artifacts according to Pialat et al.. ${ }^{34}$ Scans with at least one stack of poor quality (grade $>3$ ) were excluded from further analyses. ${ }^{34}$

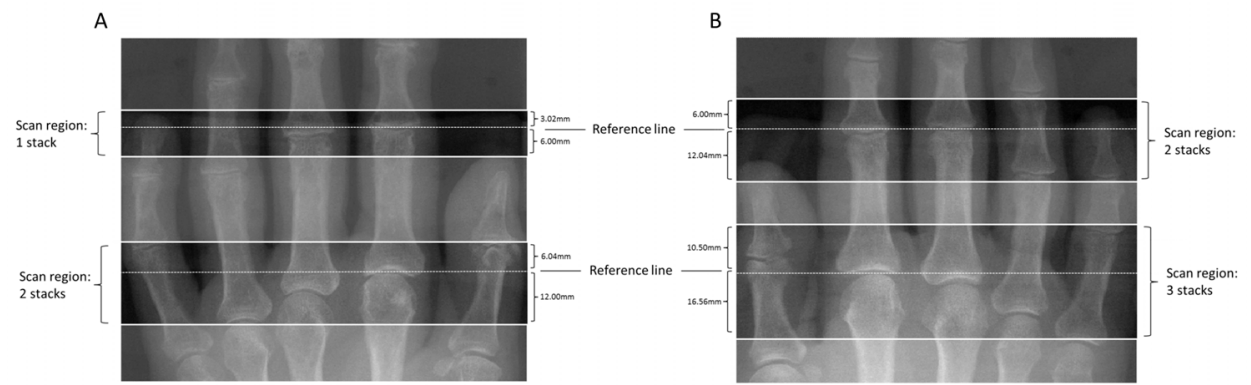

Figure 6.1. Scout views of a left (a.) and right (b.) hand, showing the regions that were scanned by HR-pQCT of the MCP and PIP scans of the first (a.) and second (b.) scan protocols. The top of the metacarpal head and proximal interphalangeal of the most distal joint (MCP2 and PIP2 in A, and MCP2 and PIP3 in B) were chosen as the landmark for the placement of the reference line. Shown is that with both scan protocols the total joint is encapsulated in the scan region. Thus, the total region where cortical interruptions can occur are included with both scan protocols. 
92 Chapter 6

\section{Image analyses}

The outer margin of the cortex was identified using an auto-contouring algorithm. ${ }^{29}$ The contours were visually inspected and, if necessary, adjusted by an operator. A segmentation to distinguish bone and non-bone was performed using the standard evaluation protocol from the manufacturer, which included Laplace-Hamming filtering and thresholding. ${ }^{35}$

\section{Cortical interruptions}

An automated algorithm for the detection of cortical interruptions and underlying trabecular bone void was applied to all binary images and is described in previous work. ${ }^{29-31}$ In short, first, a cortical mask with a constant depth of 4 voxels $(0.328 \mathrm{~mm})$ is generated based on the outer contour. Second, the bone within this mask is analyzed for cortical interruptions with a diameter of $\geq 0.41 \mathrm{~mm} .{ }^{30}$ Third, a region of interest (ROI) of approximately $4 \mathrm{~mm}$ around these cortical interruptions was identified for MCP joints and $2 \mathrm{~mm}$ for PIP joints. ${ }^{31}$ Fourth, voids in this ROI that are $\geq 0.41 \mathrm{~mm}$ in radius were selected and only those connected to the cortical interruptions were included as underlying trabecular voids. ${ }^{31}$

Per joint, the number of cortical interruptions, interruption surface area, and interruption volume (cortical interruption and trabecular void volume) were obtained (i.e. cortical interruption parameters). In addition, the proportion of joints with at least 2 cortical interruptions (proportion of joints affected) was calculated. This cut-off value was chosen because we expected at least one physiological interruption in the joints because of the presence of vascular channels. ${ }^{36}$

\section{Bone density and micro-structure parameters}

The standard and cortical evaluation protocols were used to determine the volumetric bone density and micro-structure parameters as described elsewhere. ${ }^{26,37}$ The VBMD $\left[\mathrm{mgHA} / \mathrm{cm}^{3}\right]$ was calculated for the total (Tot.BMD), trabecular (Tb.BMD) and cortical (Ct.BMD) region. Additionally, the density of the cortical bone tissue was measured (Ct.TMD). Furthermore, trabecular number (Tb.N) [ $\left.\mathrm{mm}^{-1}\right]$, thickness (Tb.Th) $[\mu \mathrm{m}]$, separation (Tb.Sp) $[\mu \mathrm{m}]$, and intra-individual distribution of separation (Tb.SpSD) [ $\mu \mathrm{m}]$ were determined to assess the trabecular compartment. The cortical thickness (Ct.Th) $[\mu \mathrm{m}]$, cortical porosity (Ct.Po) [\%], and cortical pore diameter (Ct.Po.Dm) [ $\mu \mathrm{m}]$ were determined to assess the cortical compartment.

\section{CR acquisition and scoring}

Posterior-anterior radiographs of the hands were taken from all subjects according to a standard clinical protocol. Two experienced and trained rheumatologists, which were blinded for clinical data, independently scored the radiographs of the hands in order to assess structural bone damage according to the Sharp/Van der Heijde (SvdH) method. ${ }^{38}$ 
\begin{tabular}{c|c} 
Cortical interruptions in RA and healthy subjects & \\
93
\end{tabular}

Agreement between the readers for the SvdH hand score was excellent with ICC 0.96 (95\% Cl 0.93-0.97). Mean scores of the two readers were calculated and used for the analysis. We additionally examined the SvdH erosion scores on the second and third MCP and PIP joints (radiographic damage). Radiographic damage was defined as the SvdH erosion score of the specific joint.

\section{MRI acquisition and scoring}

Second and third MCP and PIP joints of both hands were examined using a 3.0 T Achieva Philips MRI device. During the examination, the hand was fixed inside a dedicated wrist coil and the space around the hand was filled with rubber to reduce motion artifacts. Images were acquired of both hands using axial T1-weighted, axial fat-suppressed T2weighted and sagittal 3D WATSc sequences. Additional images were acquired post intravenous gadolinium (Gadovist $1.0 \mathrm{mmol} / \mathrm{ml}$ solution for injection) using axial and coronal fat-suppressed T1-weighted images.

The MRI images were independently scored by two radiologists ( $D L$ and RW), who were blinded for clinical data. Presence of erosions was evaluated per joint quadrant per bone end (thus a maximum of 8 erosions per joint could be obtained). Synovitis and BME was evaluated per joint (yes/no). Agreement per joint between the readers was good with ICC 0.75 (95\% Cl: 0.70-0.80) for the number of erosions, and $\mathrm{k} 0.71$ and 0.87 for the presence of synovitis and $\mathrm{BME}$, respectively. Consensus was reached in case of discrepancies.

\section{Statistics}

Descriptives per joint were calculated in patients with RA and healthy subjects for the proportion of joints affected, the cortical interruption-, bone density- and microstructure parameters on HR-pQCT, and for findings on CR and MRI. Stratified analyses were performed in patients with RA for hand dominance (dominant versus nondominant) and disease duration (early ( $<2$ years) versus longstanding ( $>2$ years)). Differences were tested with independent t-test, paired t-test, Mann-Whitney $U$ test or chi-squared test, as appropriate.

Possible associations were tested, first, between either structural damage on CR or structural damage and inflammatory markers on MRI, and cortical interruption parameters on HR-pQCT, second, between the presence of RA and cortical interruption parameters on HR-pQCT, and third, between either bone density or micro-structure parameters and cortical interruption parameters on HR-pQCT. Associations were calculated using repeated measures generalized estimating equations (GEE) with a negative binomial distribution, because of count data (non-normally distributed data) with overdispersion. A repeated exchangeable or unstructured working correlation structure was assumed (which fitted best) in order to adjust for within-subject correlation. All GEE analyses were performed with data from the dominant hand of each subject and were adjusted for joint (MCP or PIP), digit (second or third digit), age (years) 
and subject (patient with RA or healthy subject), if applicable. In patients with RA, all GEE analyses were repeated using data from both the dominant and non-dominant hand with, in addition, adjustment for hand dominance (dominant or non-dominant).

The added value of findings on HR-pQCT over findings on CR or MRI was tested in two additional models ('model 2' and 'model 3') in which the association between the presence of RA and cortical interruption parameters on HR-pQCT was investigated. In model 2, the analysis was additionally adjusted for findings on CR (SvdH hand score and radiographic damage) and in model 3 , the analysis was additionally adjusted for findings on MRI (number of erosions, and presence of synovitis and BME).

Statistical analyses were performed using IBM SPSS Statistics for Windows, Version 20.0 (IBM Corp., Armonk, NY) and SAS software version 9.3 (SAS Institute Inc., Cary, North Carolina, USA).

\section{RESULTS}

Two patients with RA did not have an HR-pQCT scan available and were excluded from further analyses (Figure 6.2). In one patient with RA, HR-pQCT images of the PIP joints were missing due to intolerance to long immobilization during scanning, but the remaining joints were included in the analyses (Figure 6.2). Table 6.1 shows the characteristics of the study population. Patients with RA tended to be older, and their mean (SD) disease duration was 131.7 (116.5) months.

\section{Cortical interruption parameters on HR-pQCT}

Table 6.2 shows that in the MCP joints of patients with RA, significantly more cortical interruptions were detected and in more joints, compared to healthy subjects. In addition, a significantly larger surface area and volume of cortical interruptions per joint in patients with RA compared to healthy subjects were found.

For the MCP joints, comparative stratified analyses in patients with RA showed a significant larger interruption volume in patients with longstanding RA compared to early RA patients. Furthermore, in the dominant of patients with RA, significantly more cortical interruptions and both a larger surface area and volume of interruptions could be detected compared to the non-dominant hand.

For the PIP joints, similar but smaller differences were found between patients with RA and healthy subjects, and in the stratified analyses in patients with RA. Statistical significance was only found for the proportion of joints affected between patients with RA and healthy subjects, and for the number of interruptions in the dominant compared to the non-dominant hand.

Figure $6.3 a$ shows examples of cortical interruptions detected in MCP and PIP joints of a healthy subject and a patient with RA. 


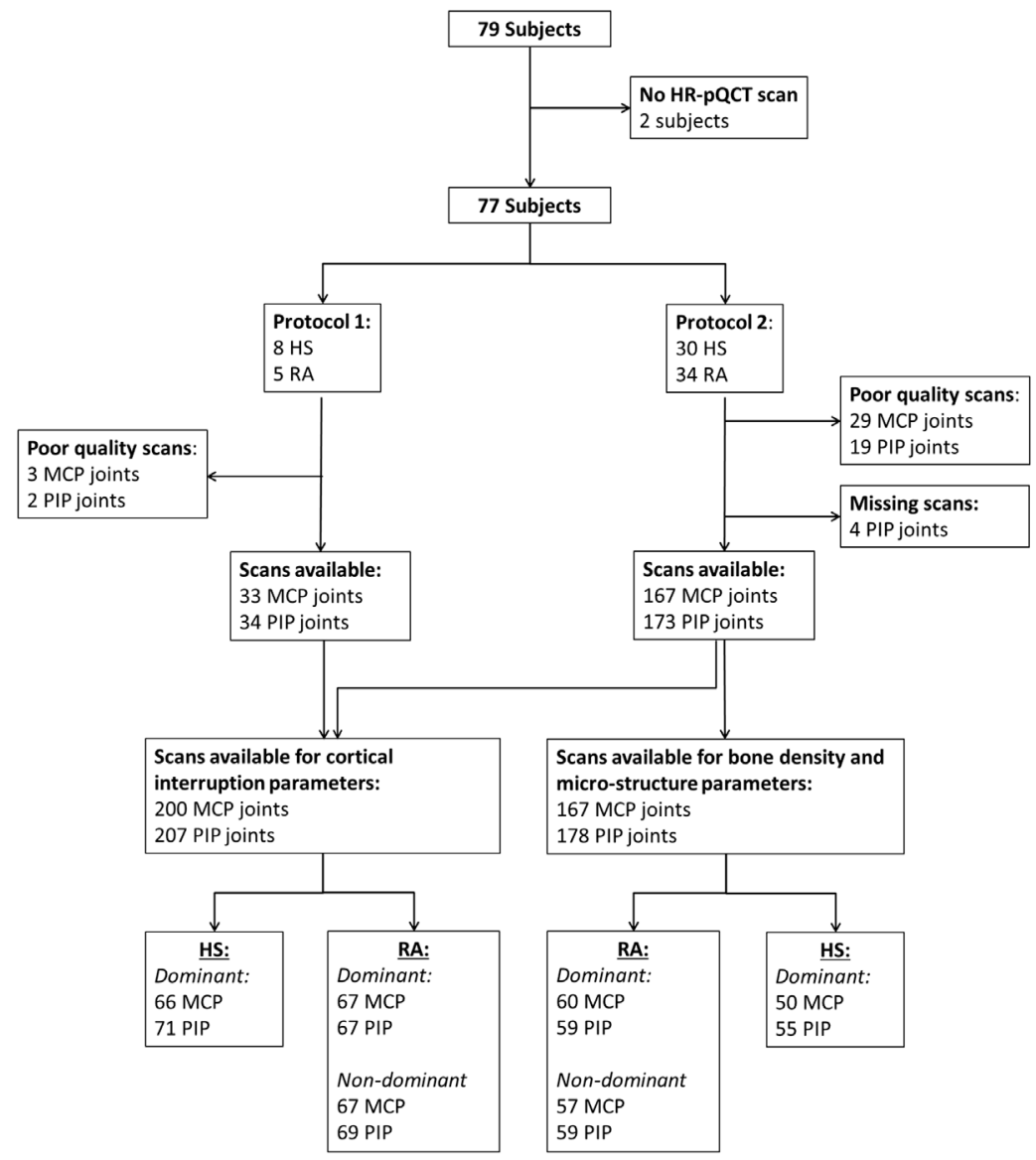

Figure 6.2. Schematic overview of the HR-pQCT scans in all subjects. Shown are the number of excluded scans because of poor quality. Additionally is shown that both scan protocols are used for analyses of the cortical interruption parameters, and only scan protocol 2 is used for analyses of the bone density and micro-structure parameters. HR-pQCT, high-resolution peripheral quantitative computed tomography; HS, healthy subject; $\mathrm{MCP}$, metacarpophalangeal joint; PIP, proximal interphalangeal joint; RA, rheumatoid arthritis.

\section{Findings on $C R$ and $M R I$}

Seventy-five baseline radiographs of the 77 subjects with an HR-pQCT scan were available and scored (39 patients with RA and 36 healthy subjects). The mean SvdH score based on two readers was significantly higher in patients with RA versus healthy subjects (mean 9.1 (SD 17.3, range 0-87.5) versus 1.1 (SD 1.8, range 0-10, $p=0.008$ ). In 10 joints from five patients with RA, radiographic damage was present in the joints specifically examined with HR-pQCT and MRI (MCP2, 3 and PIP 2, 3). In healthy subjects, no radiographic damage was observed in these joints. 
96 Chapter 6

Table 6.1. Characteristics of the participants

\begin{tabular}{lccc}
\hline & $\begin{array}{c}\text { Healthy subjects } \\
\mathrm{n}=38\end{array}$ & $\begin{array}{c}\text { Patients with RA } \\
\mathrm{n}=39\end{array}$ & $p$-value \\
\hline Age, years & $51.2(10.3)$ & $54.8(7.0)$ & 0.081 \\
Disease duration, months & $\mathrm{n} / \mathrm{a}$ & $131.7(116.5)$ & - \\
ACPA positive & $\mathrm{n} / \mathrm{a}$ & $30(77 \%)$ & \\
RF positive & $\mathrm{n} / \mathrm{a}$ & $24(59 \%)$ & \\
ACPA and RF positive & $\mathrm{n} / \mathrm{a}$ & $21(54 \%)$ & - \\
Use of DMARDs & $\mathrm{n} / \mathrm{a}$ & $34(87 \%)$ & - \\
Use of Biologicals & $\mathrm{n} / \mathrm{a}$ & $20(51 \%)$ & - \\
Use of DMARDs and biologicals & $\mathrm{n} / \mathrm{a}$ & $15(38 \%)$ & 0.624 \\
Smoking & & & \\
$\quad$ No & $22(58 \%)$ & $19(49 \%)$ & \\
Former & $11(29 \%)$ & $12(31 \%)$ & \\
Current & $5(13 \%)$ & $8(21 \%)$ & \\
\hline
\end{tabular}

All values are presented as mean (SD) or absolute number (\%).RA, rheumatoid arthritis; ACPA, anti-citrullinated protein antibody; RF, rheumatoid factor; DMARD, disease modifying anti-rheumatic drug; n/a, not applicable. In two patients, ACPA values were missing.

Seventy-five baseline MRI scans of the 77 subjects who received an HR-pQCT scan were available and scored (38 patients with RA and 37 healthy subjects). In one subject, images of four PIP joints were missing due to technical issues. Due to motion artifacts on MRI, twelve joints were not evaluable for BME and fourteen joints were not evaluable for erosion and synovitis. In patients with RA, significantly more frequently joints with erosions, BME and synovitis were found compared to healthy subjects (Supplementary Table S6.1). Longstanding RA patients had a significantly higher proportion of joints with BME compared to early RA patients. Furthermore, the mean number of erosions was significantly higher in the dominant hand compared to the non-dominant hand in patients with RA (Supplementary Table S6.1).

\section{Associations between findings on $\mathrm{CR}$ or MRI with the cortical interruption parameters on HR-pQCT}

Table 6.3 shows GEE analysis investigating associations between findings on either CR or $\mathrm{MRI}$ and cortical interruption parameters. Radiographic damage in the joint and SvdH hand score on CR were significantly associated with more cortical interruptions and both a larger surface area and volume of cortical interruptions per joint on HR-pQCT (prevalence ratios (PRs) ranging from 1.03 to 2.04, all $p<0.01$ ).

Also, the presence and number of erosions, BME and synovitis per joint on MRI were each significantly associated with more cortical interruptions and both a larger surface area and volume of cortical interruptions per joint on HR-pQCT (PRs ranging from 1.32 to 7.74, all $p<0.01$, Table 6.3).

Similar associations were found in additional analyses using data from both the dominant and non-dominant hand of patients with RA (Supplementary Table S6.2). 


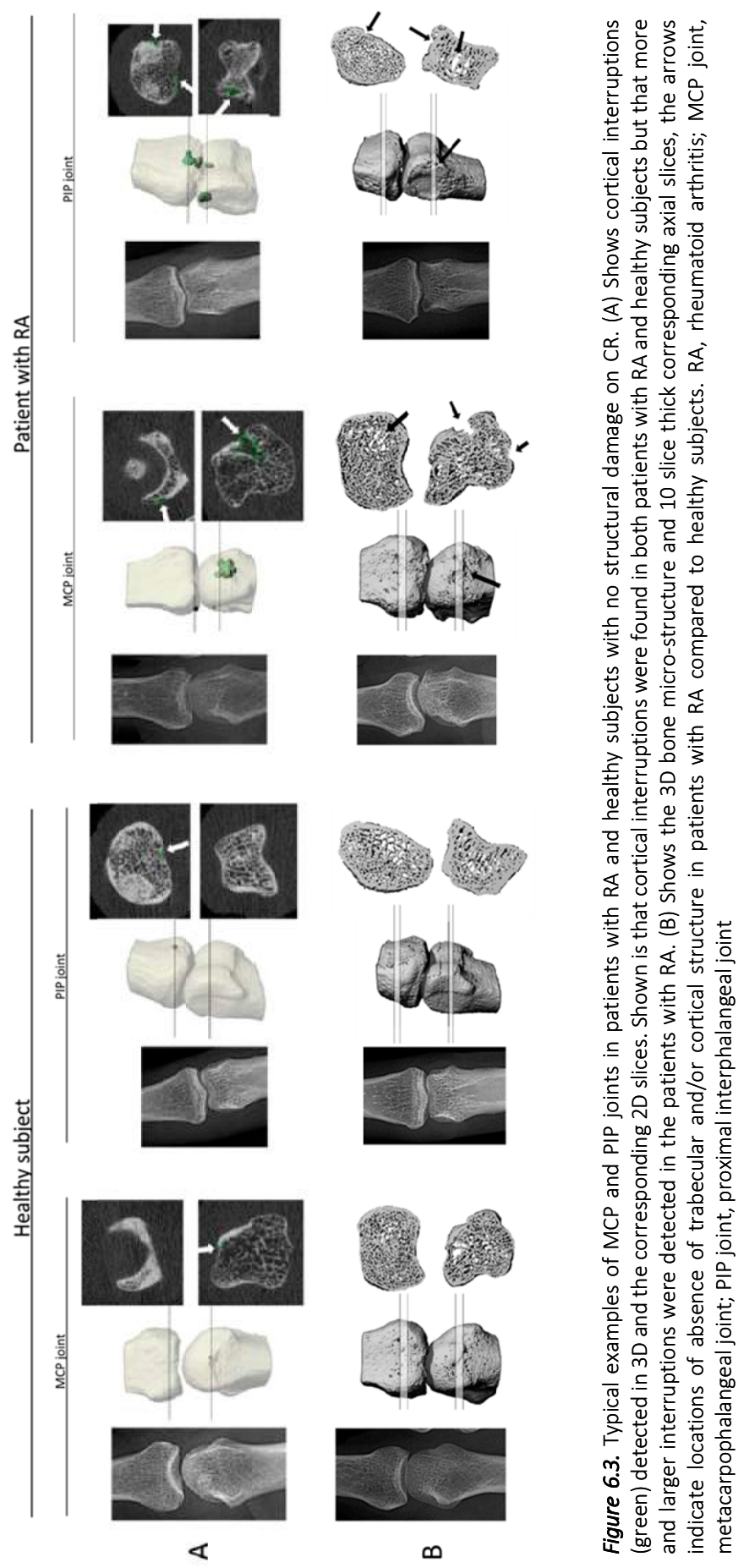


98 $\mid$ Chapter 6

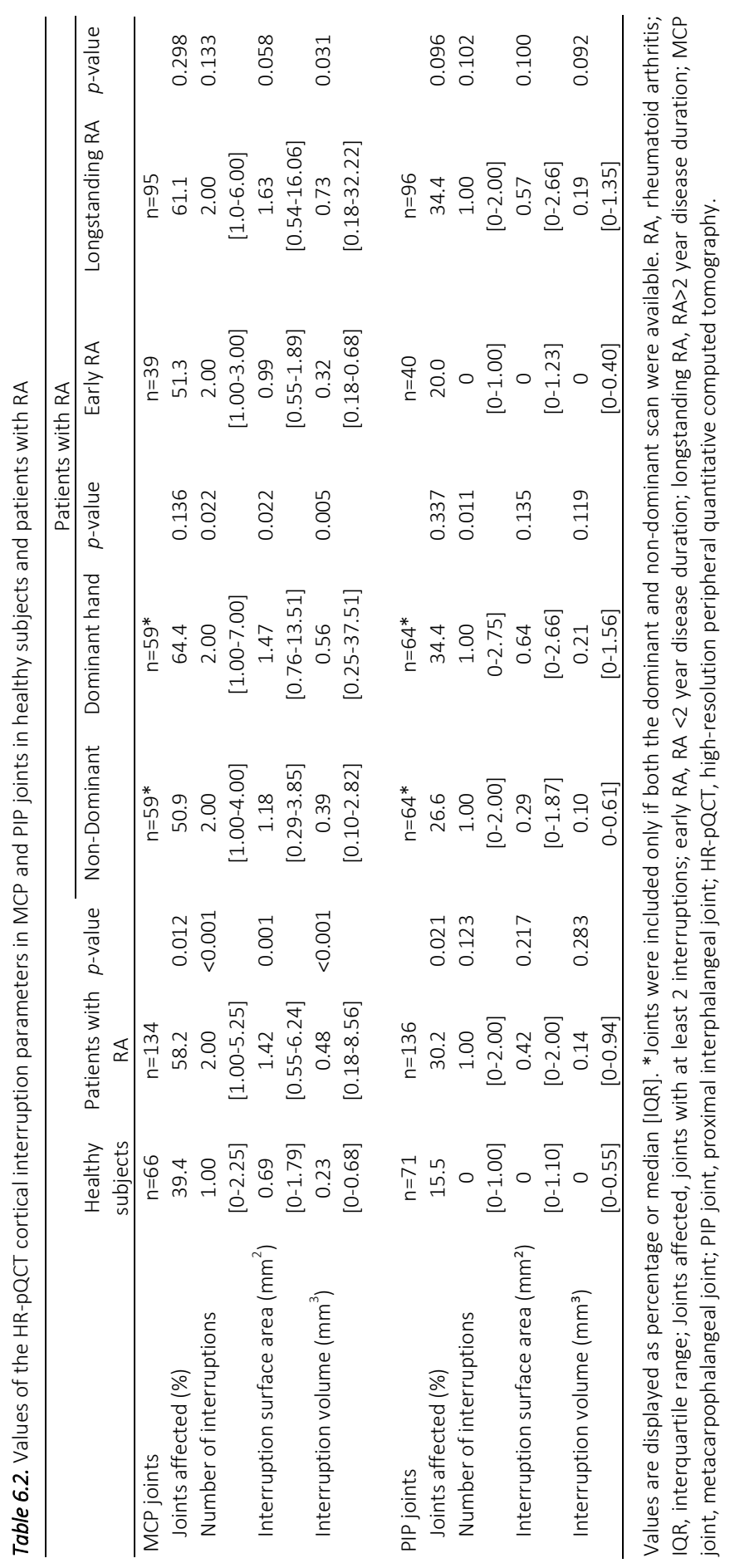


Cortical interruptions in RA and healthy subjects $\quad 99$

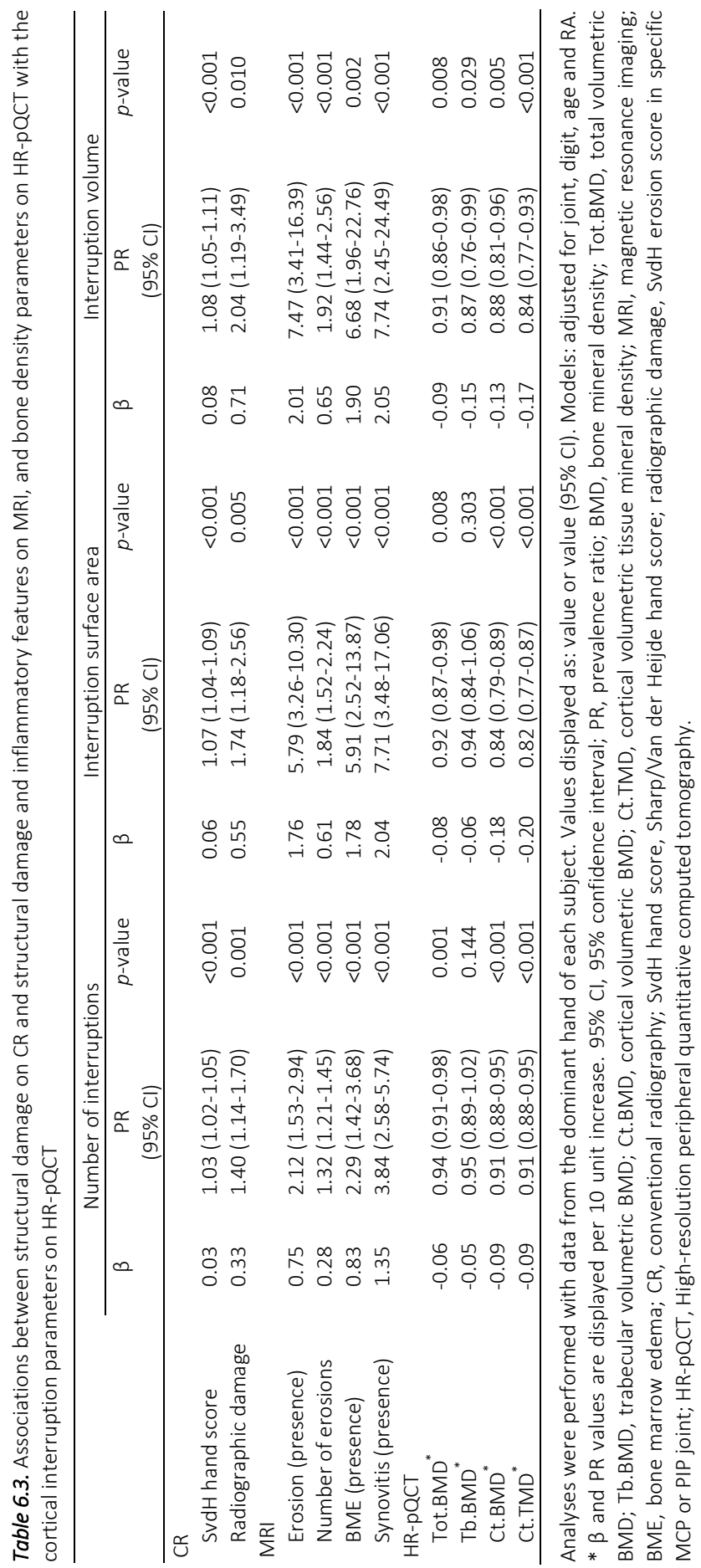




\section{Added value of HR-pQCT over CR or MRI}

The association between the presence of RA and each of the cortical interruption parameters was tested in a model with adjustment for joint, digit and age. Significant associations were found with PRs ranging from 2.33 to 5.39 (Table 6.4, model 1).

The added value of HR-pQCT over findings on CR or MRI was tested in two additional models. After adjustment for structural damage on CR (Table 6.4, model 2) or structural damage and inflammatory markers on MRI (Table 6.4, model 3), still significant associations between the presence of RA and cortical interruptions parameters were found, indicating that the HR-pQCT has an added value over findings on CR and MRI in the detection of cortical interruptions and in the distinction between patients with RA and healthy subjects.

Figure 6.3a shows examples of cortical interruptions detected on HR-pQCT in MCP and PIP joints of a patient with RA and healthy subject with no structural damage on CR. It can be seen that more and larger interruptions are detected in the patient with RA compared to the healthy subject despite no visible structural damage on CR in both subjects.

Table 6.4. Associations between the presence of RA and each of the cortical interruption parameters on HRPQCT.

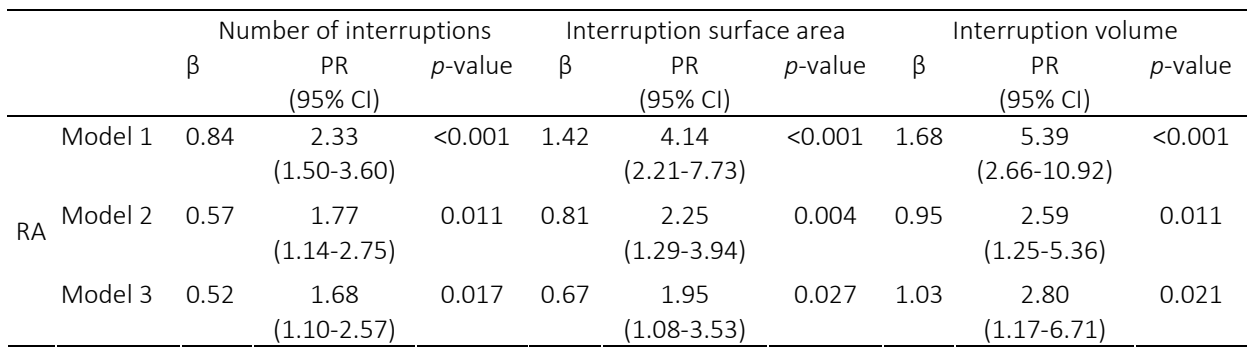

Analyses were performed with data from the dominant hand of each subject. Values displayed as: value or value $(95 \% \mathrm{Cl}$ ). Model 1: adjustment for joint, digit and age. Model 2: adjustment for joint, digit, age, radiographic damage and SvdH hand score on CR. Model 3: adjustment for joint, digit, age, erosions, BME and synovitis on MRI. 95\% Cl, 95\% confidence interval; RA, rheumatoid arthritis; PR, prevalence ratio; SvdH score, Sharp/van der Heijde score; CR, conventional radiography; MRI, magnetic resonance imaging; BME, bone marrow edema; radiographic damage, SvdH erosion score in specific MCP or PIP joint

\section{Bone density and micro-structure parameters}

In both MCP and PIP joints, we found that the VBMD was lower in patients with RA compared to healthy subjects in the total-, trabecular- and cortical region (Supplementary Tables S6.3 and S6.4). Moreover, the mineralization of the cortical bone tissue (Ct.TMD) was lower in patients with RA than in healthy subjects. Furthermore, the trabecular number (Tb.N) was lower and trabecular separation (Tb.Sp) higher with more variance in the distribution of separation (Tb.SpSD higher). In addition, a thinner cortex 
(Ct.Th lower) and smaller cortical pores (Ct.Po.Dm lower) were observed in patients with RA compared to healthy subjects (Supplementary Tables S6.3 and S6.4).

Comparative stratified analyses showed that in patients with longstanding RA compared to early RA, significantly thinner trabeculae in MCP joints were found.

Figure $6.3 \mathrm{~b}$ shows examples of trabecular and cortical bone loss in MCP and PIP joints of the dominant hand in a patient with RA compared to a healthy subject.

\section{Associations between bone density or micro-structure with the cortical interruption parameters}

A significant negative association was found between the vBMD parameters and the cortical interruption parameters per joint, except for the association between Tb.BMD and the number and surface area of interruptions (PRs ranging from: 0.82 to 0.95 per 10 unit vBMD increase, Table 6.3).

The bone micro-structure parameters did not show any significant associations with the cortical interruption parameters per joint, except for Tb.N and Tb.Sp with interruption volume (PRs: 0.21 (95\% confidence interval: 0.05-0.83) and 22.89 (1.67-313.75), respectively).

Similar associations were found in additional analyses using data from both the dominant and non-dominant hand of patients with RA (Supplementary Table S6.2).

\section{DISCUSSION}

The present study showed that more and larger cortical interruptions were found on HRpQCT in patients with RA compared to healthy subjects, and these differences were primarily found in MCP joints compared to PIP joints, as expected. ${ }^{39}$ More and larger interruptions were found in longstanding RA patients compared to early RA patients, but the difference was only significant for the interruption volume in MCP joints. Structural damage observed on CR and MRI, and markers of inflammation (BME and synovitis) on MRI were significantly associated with more cortical interruptions and both a larger surface area and volume of cortical interruptions on HR-pQCT. In addition, the presence of RA was significantly associated with the number, surface area and volume of cortical interruptions on HR-pQCT, even after adjustment for findings on CR and MRI, indicating an added value of the HR-pQCT. Moreover, bone density and micro-structural parameters were impaired in patients with RA, and a lower bone density was associated with more interruptions and both a larger surface area and volume of the interruptions on HR-pQCT. For example, every erosion on MRI was associated with a 1.32 times (32\%) increase of the number of interruptions, a 1.84 times (84\%) increase of interruption surface area and a 1.92 times (92\%) increase of interruption volume.

In the present study, we found on average a higher number of cortical interruptions in MCP joints of patients with RA than has been reported in previous studies which used 
visual scoring of HR-pQCT images (mean number of cortical interruptions was 4.3 in our study versus means ranging from 0.7 to 3.0 in previous studies). ${ }^{15-18,40,41}$ This suggests that we indeed included smaller sized interruptions with our semi-automated algorithm compared to visual scoring of interruptions on HR-pQCT.

Our observation that more small cortical interruptions could be detected on HR-pQCT in patients with RA compared to healthy subjects is in line with a previous study. ${ }^{24}$ In that study, using visual scoring on binary images, more very small cortical interruptions (with a diameter $\geq 0.082 \mathrm{~mm}$ ) were found in patients with RA compared to healthy subjects (112.9 versus 75.2$).{ }^{24}$ Small interruptions might serve as a starting point for pathological cortical interruptions. ${ }^{42}$ Monitoring these individual small cortical interruptions might be useful in understanding the pathogenesis of RA and might also be of value for evaluating the progression of structural damage or responsiveness to treatment in individual patients with RA. Previous research demonstrated for example repair processes of existing large pathological interruptions with HR-pQCT in those patients using tumor necrosis factor inhibitors (TNFi), interleukin-6 (IL-6) or receptor activator of nuclear factor kappa B ligand (RANKL) blockers. ${ }^{15,16,43}$

We also found associations between the dominant hand (compared to non-dominant hand), MCP joint (compared to PIP joint), digit 2 (compared to digit 3), a higher age, a lower $V B M D$ and more and larger interruptions in finger joints on HR-pQCT. In studies using $\mathrm{CR}$, higher SvdH scores have been reported for the dominant hand compared to the non-dominant hand. ${ }^{44,45}$ More and larger interruptions in the dominant hand support the hypothesis that mechanical factors also play a role in the development of erosions. $^{1,5}$ Using HR-pQCT imaging, Stach et al. showed that most interruptions could be found in the second MCP joint. ${ }^{11}$ Werner et al. showed that the number of small cortical interruptions in finger joints on HR-pQCT increases with increasing age. ${ }^{24}$ Last, Rossini et al. showed that a lower BMD in the hip or spine measured on dual-energy $X$-ray absorptiometry (DXA) was correlated with more interruptions on $C^{4}{ }^{46}$

Another observation in the present study was that the bone density and micro-structural parameters were impaired in patients with RA in comparison to healthy subjects. A lower bone density in both the trabecular and cortical region, fewer trabeculae (Tb.N lower, Tb.Sp and Tb.SpSD higher), and a thinner cortex was found in patients with RA. This is in agreement with previous studies investigating bone density and microstructure parameters in patients with RA compared to healthy subjects using HR$\mathrm{pQCT},{ }^{14,47}$ although the study from Fouque-Aubert et al. reported thinner trabeculae rather than fewer trabeculae, as was found in our study. ${ }^{14}$ This difference in finding might be explained by the longer scan region in our study compared to the study of Fouque-Aubert et al. (3 stacks $(27.06 \mathrm{~mm}$ ) versus 1 stack $(9.02 \mathrm{~mm})$ ), because more distally from the joint fewer trabeculae are present. ${ }^{14}$ In addition to the standard evaluation protocol, we were the first to apply the cortical evaluation protocol, which also evaluates the cortical tissue mineral density and porosity in finger joints of patients with RA in comparison to healthy subjects. The cortical tissue mineral density was significantly lower in patients with RA, meaning that the decrease in cortical bone 
density (Ct.BMD) is not only caused by the loss of cortical bone, but also by a decrease in the density of cortical bone tissue. Interestingly, in both MCP and PIP joints, the cortical porosity (Ct.Po) and cortical pore diameter (Ct.Po.Dm) were smaller in patients with RA compared to healthy subjects. This might seem contra-intuitive, but it should be noted that these Ct.Po measurements only considers intra-cortical pores, hence cortical interruptions are excluded. Because the cortical porosity and cortical pore parameter only considers intra-cortical pores, expanded intra-cortical pores are likely to become cortical interruptions, and thus are not included in these parameters. Hence, this might lead to a decrease in the cortical porosity and cortical pore diameter and increase in the cortical interruption parameters in patients with RA.

Our study has several limitations. First, we did not visually inspect and correct the inner cortical contour for determination of the cortical density and cortical micro-structural parameters. However, in a separate study in MCP joints of early arthritis patients, a high reproducibility on scan/re-scan data with in-between repositioning of these parameters without correcting the inner cortical contour was found, indicating that the determination of the inner cortical contour was reliable and only slightly dependent on repositioning and motion artifacts. ${ }^{31}$ Second, with our algorithm, the trabecular void volume underlying the cortical interruption that can be detected is limited to a depth of $4 \mathrm{~mm}$ in MCP joints and $2 \mathrm{~mm}$ in PIP joints. This means that the algorithm underestimates the volume of interruptions with a depth greater than these cut-offs (i.e. volume $>134 \mathrm{~mm}^{3}$ and $>16.8 \mathrm{~mm}^{3}$, respectively). Hence, comparability of the 3D volume of very large interruptions may be limited between our algorithm and other techniques. However, such large interruptions are not the primary focus of research with HR-pQCT, because these can also be detected by other imaging techniques with lower resolution (e.g. CR and MRI). The strength of HR-pQCT lies mainly in detection of "small" interruptions, i.e. $<10 \mathrm{~mm}^{3}$, which are regularly missed with MRI. ${ }^{48}$ Third, our study was performed using the first generation HR-pQCT. Meanwhile, the second generation HRpQCT (HR-pQCT2) has been introduced, which has a higher spatial resolution $(95 \mu \mathrm{m}$ versus $130 \mu \mathrm{m})$ and smaller voxel size $(61 \mu \mathrm{m}$ versus $82 \mu \mathrm{m})$. This higher resolution enables better distinction between bone and non-bone, which may reduce the number of falsely detected interruptions due to the partial volume effect. ${ }^{29}$ Additionally, the minimum interruption diameter will be smaller when applying 2 dilation steps ( $\geq 0.305 \mathrm{~mm}$ versus $\geq 0.410 \mathrm{~mm}$, respectively). The detection of more and smaller cortical interruptions with a possible higher validity of these interruptions may both increase the added value of HR-pQCT over findings on MRI and CR in the detection of cortical interruptions and in the distinction of patients with RA and healthy subjects

The present study has shown that our algorithm is able to detect these small cortical interruptions and showed differences in patients with RA and healthy subjects beyond structural damage on $\mathrm{CR}$, and structural damage and inflammatory markers on MRI. Only small differences between early RA and longstanding RA patients were found. This may indicate that the patients were treated well $(51 \%$ of the patients are treated with biologicals). The algorithm can best be used in arthritis patients aiming at early detection 
of structural damage. The next step is to apply this algorithm in a clinical follow-up study in order to test its potential value in monitoring patients with RA.

In conclusion, using our semi-automated algorithm, we demonstrated a higher number of interruptions, and both a larger surface area and volume of interruptions on HR-pQCT in early and longstanding RA patients in comparison to healthy subjects. Structural damage on CR and MRI and markers of inflammation (BME and synovitis) on MRI were associated with these cortical interruption parameters on HR-pQCT. HR-pQCT has shown to be of added value over findings on $C R$ and $M R I$ in the detection of cortical interruptions and in the distinction of patients with RA compared to healthy subjects. We also found an impaired bone density and micro-structure in patients with RA compared to healthy subjects, and that a lower bone density was associated with more and larger interruptions on HR-pQCT. These findings demonstrate the value of HR-pQCT imaging in the evaluation of peri-articular bone and in the assessment of (early) bone damage in addition to $C R$ and MRI in patients with RA. 


\section{REFERENCES}

1. Schett G, Gravallese E. Bone erosion in rheumatoid arthritis: mechanisms, diagnosis and treatment. Nat Rev Rheumatol. 2012;8(11):656-64.

2. Geusens P, van den Bergh J. Bone erosions in rheumatoid arthritis. Rheumatology. 2014;53(1):4-5.

3. Ejbjerg B, Narvestad E, Rostrup E, Szkudlarek M, Jacobsen S, Thomsen HS, et al. Magnetic resonance imaging of wrist and finger joints in healthy subjects occasionally shows changes resembling erosions and synovitis as seen in rheumatoid arthritis. Arthritis Rheum. 2004;50(4):1097-106.

4. Baillet A, Gaujoux-Viala C, Mouterde G, Pham T, Tebib J, Saraux A, et al. Comparison of the efficacy of sonography, magnetic resonance imaging and conventional radiography for the detection of bone erosions in rheumatoid arthritis patients: a systematic review and meta-analysis. Rheumatology. 2011;50(6):1137-47.

5. Geusens P, Chapurlat R, Schett G, Ghasem-Zadeh A, Seeman E, de Jong J, et al. High-resolution in vivo imaging of bone and joints: a window to microarchitecture. Nat Rev Rheumatol. 2014;10(5):304-13.

6. Dohn UM, Ejbjerg BJ, Hasselquist M, Narvestad E, Moller J, Thomsen HS, et al. Detection of bone erosions in rheumatoid arthritis wrist joints with magnetic resonance imaging, computed tomography and radiography. Arthritis Res Ther. 2008;10(1):R25.

7. Dohn UM, Ejbjerg B, Boonen A, Hetland ML, Hansen MS, Knudsen LS, et al. No overall progression and occasional repair of erosions despite persistent inflammation in adalimumab-treated rheumatoid arthritis patients: results from a longitudinal comparative MRI, ultrasonography, CT and radiography study. Ann Rheum Dis. 2011;70(2):252-8.

8. Gandjbakhch F, Foltz V, Mallet A, Bourgeois P, Fautrel B. Bone marrow oedema predicts structural progression in a 1-year follow-up of 85 patients with RA in remission or with low disease activity with low-field MRI. Ann Rheum Dis. 2011;70(12):2159-62.

9. Hetland ML, Ejbjerg B, Horslev-Petersen K, Jacobsen S, Vestergaard A, Jurik AG, et al. MRI bone oedema is the strongest predictor of subsequent radiographic progression in early rheumatoid arthritis. Results from a 2-year randomised controlled trial (CIMESTRA). Ann Rheum Dis. 2009;68(3):384-90.

10. Peterfy C, Strand V, Tian L, Ostergaard M, Lu Y, DiCarlo J, et al. Short-term changes on MRI predict longterm changes on radiography in rheumatoid arthritis: an analysis by an OMERACT Task Force of pooled data from four randomised controlled trials. Ann Rheum Dis. 2017;76(6):992-7.

11. Stach CM, Bauerle M, Englbrecht M, Kronke G, Engelke K, Manger B, et al. Periarticular bone structure in rheumatoid arthritis patients and healthy individuals assessed by high-resolution computed tomography. Arthritis Rheum. 2010;62(2):330-9.

12. Regensburger A, Rech J, Englbrecht M, Finzel S, Kraus S, Hecht K, et al. A comparative analysis of magnetic resonance imaging and high-resolution peripheral quantitative computed tomography of the hand for the detection of erosion repair in rheumatoid arthritis. Rheumatology (Oxford). 2015;54(9):1573-81.

13. Lee $\mathrm{CH}$, Srikhum W, Burghardt AJ, Virayavanich W, Imboden JB, Link TM, et al. Correlation of structural abnormalities of the wrist and metacarpophalangeal joints evaluated by high-resolution peripheral quantitative computed tomography, 3 Tesla magnetic resonance imaging and conventional radiographs in rheumatoid arthritis. Int J Rheum Dis. 2015;18(6):628-39.

14. Fouque-Aubert A, Boutroy S, Marotte H, Vilayphiou N, Bacchetta J, Miossec P, et al. Assessment of hand bone loss in rheumatoid arthritis by high-resolution peripheral quantitative CT. Ann Rheum Dis. 2010;69(9):1671-6.

15. Finzel S, Rech J, Schmidt S, Engelke K, Englbrecht M, Stach C, et al. Repair of bone erosions in rheumatoid arthritis treated with tumour necrosis factor inhibitors is based on bone apposition at the base of the erosion. Ann Rheum Dis. 2011;70(9):1587-93.

16. Finzel S, Rech J, Schmidt S, Engelke K, Englbrecht M, Schett G. Interleukin-6 receptor blockade induces limited repair of bone erosions in rheumatoid arthritis: a micro CT study. Ann Rheum Dis. 2013;72(3): 396-400.

17. Srikhum W, Virayavanich W, Burghardt AJ, Yu A, Link TM, Imboden JB, et al. Quantitative and semiquantitative bone erosion assessment on high-resolution peripheral quantitative computed tomography in rheumatoid arthritis. J Rheumatol. 2013;40(4):408-16. 
18. Topfer D, Finzel S, Museyko O, Schett G, Engelke K. Segmentation and quantification of bone erosions in high-resolution peripheral quantitative computed tomography datasets of the metacarpophalangeal joints of patients with rheumatoid arthritis. Rheumatology. 2014;53(1):65-71.

19. Topfer D, Gerner B, Finzel S, Kraus S, Museyko O, Schett G, et al. Automated three-dimensional registration of high-resolution peripheral quantitative computed tomography data to quantify size and shape changes of arthritic bone erosions. Rheumatology. 2015;54(12):2171-80.

20. Barnabe C, Toepfer D, Marotte H, Hauge EM, Scharmga A, Kocijan R, et al. Definition for Rheumatoid Arthritis Erosions Imaged with High Resolution Peripheral Quantitative Computed Tomography and Interreader Reliability for Detection and Measurement. J Rheumatol. 2016;43(10):1935-40.

21. Scharmga A, Peters M, van Tubergen A, van den Bergh J, Barnabe C, Finzel S, et al. Heterogeneity of Cortical Breaks in Hand Joints of Patients with Rheumatoid Arthritis and Healthy Controls Imaged by Highresolution Peripheral Quantitative Computed Tomography. J Rheumatol. 2016;43(10):1914-20.

22. Binks DA, Gravallese EM, Bergin D, Hodgson RJ, Tan AL, Matzelle MM, et al. Role of vascular channels as a novel mechanism for subchondral bone damage at cruciate ligament entheses in osteoarthritis and inflammatory arthritis. Ann Rheum Dis. 2015 Jan;74(1):196-203.

23. Schett G, Stolina M, Bolon B, Middleton S, Adlam M, Brown H, et al. Analysis of the kinetics of osteoclastogenesis in arthritic rats. Arthritis Rheum. 2005;52(10):3192-201.

24. Werner D, Simon D, Englbrecht M, Stemmler F, Simon C, Berlin A, et al. Rheumatoid arthritis is characterized by early changes of the cortical micro-channel (CoMiC) system in the bare area of the joints. Arthritis Rheum. 2017;69(8):1580-7.

25. Simon D, Kleyer A, Stemmler F, Simon C, Berlin A, Hueber AJ, et al. Age- and sex-dependent changes of intra-articular cortical and trabecular bone structure and the effects of rheumatoid arthritis. J Bone Miner Res. 2017;32(4):722-30.

26. Boutroy S, Bouxsein ML, Munoz F, Delmas PD. In vivo assessment of trabecular bone microarchitecture by high-resolution peripheral quantitative computed tomography. J Clin Endocrinol Metab. 2005;90(12):6508-15.

27. Zebaze R, Seeman E. Cortical bone: a challenging geography. J Bone Miner Res. 2015;30(1):24-9.

28. Scharmga A, Peters $M$, van Tubergen A, van den Bergh J, de Jong J, Loeffen $D$, et al. Visual detection of cortical breaks in hand joints: reliability and validity of high-resolution peripheral quantitative CT compared to microCT. BMC Musculoskelet Disord. 2016;17(1):271.

29. Peters M, Scharmga A, de Jong J, van Tubergen A, Geusens P, Arts JJ, et al. An automated algorithm for the detection of cortical interruptions on high resolution peripheral quantitative computed tomography images of finger joints. PloS One. 2017;12(4):e0175829.

30. Peters M, Scharmga A, van Tubergen A, Arts J, Loeffen D, Weijers R, et al. The Reliability of a Semiautomated Algorithm for Detection of Cortical Interruptions in Finger Joints on High Resolution CT Compared to MicroCT. Calcif Tissue Int. 2017;101(2):132-40.

31. Peters $M$, de Jong J, Scharmga A, van Tubergen A, Loeffen D, Weijers R, et al. The reproducibility of a semi-automatic algorithm in the detection of cortical breaks and adjacent trabecular bone loss in scanrescan data from patients with early arthritis [abstract]. Arthritis Rheum. 2016;68 (suppl 10).

32. Aletaha D, Neogi T, Silman AJ, Funovits J, Felson DT, Bingham CO, 3rd, et al. 2010 Rheumatoid arthritis classification criteria: an American College of Rheumatology/European League Against Rheumatism collaborative initiative. Arthritis Rheum. 2010;62(9):2569-81.

33. Barnabe C, Feehan L. High-resolution peripheral quantitative computed tomography imaging protocol for metacarpophalangeal joints in inflammatory arthritis: the SPECTRA collaboration. J Rheumatol. 2012;39(7):1494-5.

34. Pialat JB, Burghardt AJ, Sode M, Link TM, Majumdar S. Visual grading of motion induced image degradation in high resolution peripheral computed tomography: impact of image quality on measures of bone density and micro-architecture. Bone. 2012;50(1):111-8.

35. Laib A, Hauselmann HJ, Ruegsegger P. In vivo high resolution 3D-QCT of the human forearm. Technol Health Care. 1998;6(5-6):329-37.

36. Scharmga A, Keller KK, Peters M, van Tubergen A, van den Bergh JP, van Rietbergen B, et al. Vascular channels in metacarpophalangeal joints: a comparative histologic and high-resolution imaging study. Sci Rep. 2017;7(1):8966.

37. Burghardt AJ, Buie HR, Laib A, Majumdar S, Boyd SK. Reproducibility of direct quantitative measures of cortical bone microarchitecture of the distal radius and tibia by HR-pQCT. Bone. 2010;47(3):519-28. 
38. van der Heijde D. How to read radiographs according to the Sharp/van der Heijde method. J Rheumatol. 2000;27(1):261-3.

39. van der Heijde DM, van Leeuwen MA, van Riel PL, Koster AM, van 't Hof MA, van Rijswijk MH, et al. Biannual radiographic assessments of hands and feet in a three-year prospective followup of patients with early rheumatoid arthritis. Arthritis Rheum. 1992;35(1):26-34.

40. Finzel S, Englbrecht M, Engelke K, Stach C, Schett G. A comparative study of periarticular bone lesions in rheumatoid arthritis and psoriatic arthritis. Ann Rheum Dis. 2011;70(1):122-7.

41. Barnabe C, Szabo E, Martin L, Boyd SK, Barr SG. Quantification of small joint space width, periarticular bone microstructure and erosions using high-resolution peripheral quantitative computed tomography in rheumatoid arthritis. Clin Exp Rheumatol. 2013;31(2):243-50.

42. Schett G, Gravallese E. Bone erosion in rheumatoid arthritis: mechanisms, diagnosis and treatment. Nat Rev Rheumatol. 2012;8(11):656-64.

43. Yue J, Griffith JF, Xiao F, Shi L, Wang D, Shen J, et al. Repair of bone erosion in rheumatoid arthritis by denosumab: A high-resolution peripheral quantitative computed tomography study. Arthritis Care Res (Hoboken). 2017;69(8):1156-63.

44. Koh JH, Jung SM, Lee JJ, Kang KY, Kwok SK, Park SH, et al. Radiographic Structural Damage Is Worse in the Dominant than the Non-Dominant Hand in Individuals with Early Rheumatoid Arthritis. PLoS One. 2015;10(8):e0135409.

45. Owsianik WD, Kundi A, Whitehead JN, Kraag GR, Goldsmith C. Radiological articular involvement in the dominant hand in rheumatoid arthritis. Ann Rheum Dis. 1980;39(5):508-10.

46. Rossini M, Bagnato G, Frediani B, lagnocco A, G LAM, Minisola G, et al. Relationship of focal erosions, bone mineral density, and parathyroid hormone in rheumatoid arthritis. J Rheumatol. 2011;38(6): 997-1002.

47. Yang H, Yu A, Burghardt AJ, Virayavanich W, Link TM, Imboden JB, et al. Quantitative characterization of metacarpal and radial bone in rheumatoid arthritis using high resolution- peripheral quantitative computed tomography. Int J Rheum Dis. 2017;20(3):353-62.

48. Albrecht A, Finzel S, Englbrecht M, Rech J, Hueber A, Schlechtweg P, et al. The structural basis of MRI bone erosions: an assessment by microCT. Ann Rheum Dis. 2013;72(8):1351-7. 


\section{SUPPLEMENTARY FILES}

Table S6.1. MRI scoring in MCP and PIP joints of healthy subjects and patients with RA.

\begin{tabular}{|c|c|c|c|c|c|c|c|c|c|}
\hline & \multirow[b]{2}{*}{$\begin{array}{c}\text { Healthy } \\
\text { subjects } \\
n=38\end{array}$} & \multirow[b]{2}{*}{$\begin{array}{c}\text { Patients } \\
\text { with RA } \\
n=39\end{array}$} & \multirow[b]{2}{*}{$p$-value } & \multicolumn{6}{|c|}{ Patients with RA } \\
\hline & & & & $\begin{array}{l}\text { Non-dominant } \\
\text { hand } \\
n=39\end{array}$ & $\begin{array}{c}\text { Dominant } \\
\text { hand } \\
n=39\end{array}$ & $p$-value & $\begin{array}{c}\text { Early RA } \\
n=10\end{array}$ & $\begin{array}{c}\text { Longstanding } \\
\text { RA } \\
n=29\end{array}$ & $\begin{array}{c}p- \\
\text { value }\end{array}$ \\
\hline $\begin{array}{l}\text { Number of joints } \\
\text { evaluable for erosions }\end{array}$ & 144 & 286 & & 138 & 148 & & 64 & 222 & \\
\hline $\begin{array}{l}\text { Proportion of joints } \\
\text { with erosions (\%) }\end{array}$ & 17.4 & 28.7 & 0.010 & 23.9 & 33.1 & 0.086 & 21.9 & 30.6 & 0.172 \\
\hline $\begin{array}{l}\text { Mean number of } \\
\text { erosions per joint }\end{array}$ & $\begin{array}{c}0.20 \\
(0.48)\end{array}$ & $\begin{array}{c}0.63 \\
(1.36)\end{array}$ & 0.081 & $\begin{array}{c}0.50 \\
(1.22)\end{array}$ & $\begin{array}{c}0.74 \\
(1.48)\end{array}$ & 0.012 & $\begin{array}{l}0.33 \\
(0.74)\end{array}$ & $\begin{array}{c}0.72 \\
(1.49)\end{array}$ & 0.119 \\
\hline $\begin{array}{l}\text { Number of joints } \\
\text { evaluable for BME }\end{array}$ & 144 & 284 & & 136 & 148 & & 64 & 220 & \\
\hline $\begin{array}{l}\text { Proportion of joints } \\
\text { with BME (\%) }\end{array}$ & 1.4 & 9.9 & 0.001 & 7.4 & 12.2 & 0.174 & 1.6 & 12.3 & 0.011 \\
\hline $\begin{array}{l}\text { Number of joints } \\
\text { evaluable for synovitis }\end{array}$ & 148 & 296 & & 148 & 148 & & 68 & 228 & \\
\hline $\begin{array}{l}\text { Proportion of joints } \\
\text { with synovitis (\%) }\end{array}$ & 2.0 & 10.8 & 0.001 & 7.4 & 14.2 & 0.061 & 4.4 & 12.7 & 0.053 \\
\hline
\end{tabular}

Values are displayed as percentage or mean (SD). RA, rheumatoid arthritis; BME, bone marrow edema; SD, standard deviation; early RA, RA $<2$ years disease duration; longstanding RA, RA $>2$ years disease duration. * Significantly different from healthy subjects. " four PIP joints missing in one patient with RA. Due to motion artifacts, twelve joints were not evaluable for BME and fourteen joints were not evaluable for synovitis and erosion 
Table S6.2. Associations between structural damage on CR and structural damage and inflammatory features on MRI, and bone density parameters on HR-pQCT with the cortical interruption parameters on HR-pQCT in patients with RA

\begin{tabular}{|c|c|c|c|c|c|c|c|c|c|}
\hline & \multicolumn{3}{|c|}{ Number of interruptions } & \multicolumn{3}{|c|}{ Interruption surface area } & \multicolumn{3}{|c|}{ Interruption volume } \\
\hline & $\beta$ & $\begin{array}{c}\mathrm{PR} \\
(95 \% \mathrm{Cl})\end{array}$ & $p$-value & $\beta$ & $\begin{array}{c}\text { PR } \\
(95 \% \mathrm{Cl})\end{array}$ & $p$-value & $\beta$ & $\begin{array}{c}\mathrm{PR} \\
(95 \% \mathrm{Cl})\end{array}$ & $\begin{array}{c}p- \\
\text { value }\end{array}$ \\
\hline \multicolumn{10}{|l|}{$C R$} \\
\hline SvdH hand score & 0.02 & $\begin{array}{c}1.02 \\
(1.00-1.04)\end{array}$ & 0.014 & 0.04 & $\begin{array}{c}1.04 \\
(1.01-1.07)\end{array}$ & 0.007 & 0.05 & $\begin{array}{c}1.05 \\
(1.01-1.09)\end{array}$ & 0.012 \\
\hline Radiographic damage & 0.21 & $\begin{array}{c}1.23 \\
(1.00-1.52)\end{array}$ & 0.047 & 0.43 & $\begin{array}{c}1.53 \\
(1.02-2.30)\end{array}$ & 0.039 & 0.55 & $\begin{array}{c}1.74 \\
(0.91-3.32)\end{array}$ & 0.096 \\
\hline \multicolumn{10}{|l|}{ MRI } \\
\hline Erosion (presence) & 0.83 & $\begin{array}{c}2.29 \\
(1.63-3.21)\end{array}$ & $<0.001$ & 1.89 & $\begin{array}{c}6.62 \\
(3.80-11.55)\end{array}$ & $<0.001$ & 3.14 & $\begin{array}{c}23.06 \\
(6.17-86.19)\end{array}$ & $<0.001$ \\
\hline Number of erosions & 0.28 & $\begin{array}{c}1.32 \\
(1.21-1.44)\end{array}$ & $<0.001$ & 0.59 & $\begin{array}{c}1.80 \\
(1.53-2.12)\end{array}$ & $<0.001$ & 0.65 & $\begin{array}{c}1.92 \\
(1.44-2.56)\end{array}$ & $<0.001$ \\
\hline BME (presence) & 0.71 & $\begin{array}{c}2.03 \\
(1.34-3.07)\end{array}$ & $<0.001$ & 1.70 & $\begin{array}{c}5.47 \\
(2.66-11.25)\end{array}$ & $<0.001$ & 1.77 & $\begin{array}{c}5.86 \\
(1.72-19.96)\end{array}$ & 0.005 \\
\hline Synovitis (presence) & 0.91 & $\begin{array}{c}2.48 \\
(1.68-3.66)\end{array}$ & $<0.001$ & 1.90 & $\begin{array}{c}6.69 \\
(3.30-13.54)\end{array}$ & $<0.001$ & 2.05 & $\begin{array}{c}7.75 \\
(2.21-27.26)\end{array}$ & 0.001 \\
\hline \multicolumn{10}{|l|}{ HR-pQCT } \\
\hline Tot.BMD* & -0.06 & $\begin{array}{c}0.94 \\
(0.91-0.97)\end{array}$ & $<0.001$ & -0.09 & $\begin{array}{c}0.91 \\
(0.87-0.96)\end{array}$ & $<0.001$ & -0.12 & $\begin{array}{c}0.89 \\
(0.84-0.94)\end{array}$ & $<0.001$ \\
\hline Tb.BMD* & -0.07 & $\begin{array}{c}0.93 \\
(0.88-1.00)\end{array}$ & 0.039 & -0.12 & $\begin{array}{c}0.89 \\
(0.80-0.99)\end{array}$ & 0.030 & -0.19 & $\begin{array}{c}0.83 \\
(0.73-0.93)\end{array}$ & 0.002 \\
\hline Ct.BMD* & -0.07 & $\begin{array}{c}0.93 \\
(0.90-0.97)\end{array}$ & $<0.001$ & -0.14 & $\begin{array}{c}0.87 \\
(0.81-0.92)\end{array}$ & $<0.001$ & -0.19 & $\begin{array}{c}0.82 \\
(0.75-0.90)\end{array}$ & $<0.001$ \\
\hline Ct.TMD* & -0.07 & $\begin{array}{c}0.93 \\
(0.89-0.97) \\
\end{array}$ & 0.001 & -0.15 & $\begin{array}{c}0.86 \\
(0.80-0.92) \\
\end{array}$ & $<0.001$ & -0.23 & $\begin{array}{c}0.79 \\
(0.72-0.87) \\
\end{array}$ & $<0.001$ \\
\hline
\end{tabular}

Analyses were performed with data from both hands of patients with RA. Values displayed as: value or value $(95 \% \mathrm{Cl})$. Models: adjusted for hand dominance, joint, digit, age and disease duration (early or longstanding RA). ${ }^{*} \beta$ and IRR values are displayed per 10 unit increase. $95 \% \mathrm{Cl}, 95 \%$ confidence interval; IRR, incidence rate ratio; $\mathrm{BMD}$, bone mineral density; Tot.BMD, total volumetric BMD; Tb.BMD, trabecular volumetric BMD; Ct.BMD, cortical volumetric BMD; Ct.TMD, cortical volumetric tissue mineral density; MRI, magnetic resonance imaging; $\mathrm{BME}$, bone marrow edema; CR, conventional radiography; SvdH, Sharp/Van der Heijde score; radiographic damage, SvdH erosion score in specific joint; HR-pQCT, High-resolution peripheral quantitative computed tomography 
$110 \mid$ Chapter 6

Table S6.3. HR-pQCT bone density, and micro-structure parameters in MCP joints of healthy subjects and patients with RA

\begin{tabular}{|c|c|c|c|c|c|c|c|c|c|}
\hline \multirow{3}{*}{ MCP joints } & \multirow{3}{*}{$\begin{array}{l}\text { Healthy } \\
\text { subjects } \\
n=50\end{array}$} & \multirow{3}{*}{$\begin{array}{l}\begin{array}{l}\text { Patients } \\
\text { with RA } \\
n=117\end{array} \\
\end{array}$} & \multirow{3}{*}{$p$-value } & \multicolumn{6}{|c|}{ Patients with RA } \\
\hline & & & & $\begin{array}{l}\text { non- } \\
\text { dominant } \\
\text { hand }\end{array}$ & $\begin{array}{l}\text { Dominant } \\
\text { hand }\end{array}$ & $p$-value & early RA & $\begin{array}{c}\text { longstanding } \\
\text { RA }\end{array}$ & $p$-value \\
\hline & & & & $\mathrm{n}=52 *$ & $n=52 *$ & & $n=31$ & $n=86$ & \\
\hline \multicolumn{10}{|l|}{ Bone density parameters } \\
\hline Tot.BMD (mg HA/ $/ \mathrm{cm}^{3}$ ) & $\begin{array}{l}327.3 \\
(35.3)\end{array}$ & $\begin{array}{l}286.3 \\
(57.1)\end{array}$ & $<0.001$ & $\begin{array}{l}283.8 \\
(52.3)\end{array}$ & $\begin{array}{l}287.8 \\
(56.3)\end{array}$ & 0.357 & $\begin{array}{l}295.8 \\
(38.9)\end{array}$ & $\begin{array}{l}282.8 \\
(62.2)\end{array}$ & 0.183 \\
\hline Tb.BMD (mg HA/cm³) & $\begin{array}{l}202.1 \\
(20.6)\end{array}$ & $\begin{array}{l}178.0 \\
(35.6)\end{array}$ & $<0.001$ & $\begin{array}{l}177.4 \\
(32.5)\end{array}$ & $\begin{array}{l}177.9 \\
(34.6)\end{array}$ & 0.873 & $\begin{array}{l}185.0 \\
(21.6)\end{array}$ & $\begin{array}{l}175.4 \\
(39.2)\end{array}$ & 0.097 \\
\hline Ct.BMD (mg HA/cm³) & $\begin{array}{l}835.8 \\
(39.8)\end{array}$ & $\begin{array}{l}797.8 \\
(52.5)\end{array}$ & $<0.001$ & $\begin{array}{l}795.7 \\
(49.8)\end{array}$ & $\begin{array}{l}800.0 \\
(54.8)\end{array}$ & 0.262 & $\begin{array}{l}806.2 \\
(59.9)\end{array}$ & $\begin{array}{l}794.7 \\
(49.6)\end{array}$ & 0.296 \\
\hline Ct.TMD (mg HA/ $\mathrm{cm}^{3}$ ) & $\begin{array}{l}909.8 \\
(32.0)\end{array}$ & $\begin{array}{l}878.8 \\
(48.8)\end{array}$ & $<0.001$ & $\begin{array}{l}877.3 \\
(47.0)\end{array}$ & $\begin{array}{l}881.1 \\
(49.4)\end{array}$ & 0.286 & $\begin{array}{l}886.1 \\
(54.1)\end{array}$ & $\begin{array}{l}876.2 \\
(46.7)\end{array}$ & 0.335 \\
\hline \multicolumn{10}{|c|}{ Bone micro-structure parameters } \\
\hline Tb.N $\left(\mathrm{mm}^{-1}\right)$ & $\begin{array}{c}1.68 \\
(0.31)\end{array}$ & $\begin{array}{c}1.47 \\
(0.35)\end{array}$ & $<0.001$ & $\begin{array}{c}1.47 \\
(0.36)\end{array}$ & $\begin{array}{c}1.43 \\
(0.34)\end{array}$ & 0.242 & $\begin{array}{c}1.45 \\
(0.29)\end{array}$ & $\begin{array}{c}1.47 \\
(0.37)\end{array}$ & 0.772 \\
\hline Tb.Th $(\mu \mathrm{m})$ & $\begin{array}{l}102.3 \\
(15.3)\end{array}$ & $\begin{array}{l}103.4 \\
(17.4)\end{array}$ & 0.703 & $\begin{array}{l}103.2 \\
(17.3)\end{array}$ & $\begin{array}{l}105.2 \\
(16.7)\end{array}$ & 0.216 & $\begin{array}{l}109.1 \\
(17.8)\end{array}$ & $\begin{array}{l}101.3 \\
(16.9)\end{array}$ & 0.033 \\
\hline Tb.Sp $(\mu \mathrm{m})$ & $\begin{array}{c}513.9 \\
(116.9)\end{array}$ & $\begin{array}{c}624.5 \\
(196.9)\end{array}$ & $<0.001$ & $\begin{array}{c}621.4 \\
(184.2)\end{array}$ & $\begin{array}{c}637.2 \\
(213.7)\end{array}$ & 0.434 & $\begin{array}{c}608.4 \\
(132.3)\end{array}$ & $\begin{array}{c}630.3 \\
(215.9)\end{array}$ & 0.511 \\
\hline Tb.SpSD $(\mu \mathrm{m})$ & $\begin{array}{c}550.6 \\
(287.0)\end{array}$ & $\begin{array}{c}739.6 \\
(366.5)\end{array}$ & 0.001 & $\begin{array}{c}733.7 \\
(364.8)\end{array}$ & $\begin{array}{c}762.6 \\
(380.7)\end{array}$ & 0.437 & $\begin{array}{c}728.5 \\
(306.1)\end{array}$ & $\begin{array}{c}743.6 \\
(387.5)\end{array}$ & 0.845 \\
\hline Ct.Th $(\mu \mathrm{m})$ & $\begin{array}{c}664.5 \\
(111.5)\end{array}$ & $\begin{array}{c}591.6 \\
(122.5)\end{array}$ & $<0.001$ & $\begin{array}{c}579.5 \\
(112.0)\end{array}$ & $\begin{array}{c}601.4 \\
(127.2)\end{array}$ & 0.124 & $\begin{array}{c}599.2 \\
(105.4)\end{array}$ & $\begin{array}{c}588.9 \\
(128.6)\end{array}$ & 0.689 \\
\hline Ct.Po (\%) & $\begin{array}{c}5.37 \\
(1.42)\end{array}$ & $\begin{array}{c}5.64 \\
(1.52)\end{array}$ & 0.284 & $\begin{array}{c}5.63 \\
(1.24)\end{array}$ & $\begin{array}{c}5.73 \\
(1.77)\end{array}$ & 0.629 & $\begin{array}{c}5.76 \\
(1.70)\end{array}$ & $\begin{array}{c}5.59 \\
(1.45)\end{array}$ & 0.605 \\
\hline Ct.Po.Dm $(\mu \mathrm{m})$ & $\begin{array}{c}212.3 \\
(137.7)\end{array}$ & $\begin{array}{l}190.8 \\
(22.4)\end{array}$ & 0.101 & $\begin{array}{l}192.5 \\
(21.7)\end{array}$ & $\begin{array}{l}192.0 \\
(24.8)\end{array}$ & 0.899 & $\begin{array}{l}192.7 \\
(21.4)\end{array}$ & $\begin{array}{l}190.1 \\
(22.9)\end{array}$ & 0.586 \\
\hline
\end{tabular}

Values are displayed as mean (SD). *Joints were included only if both the dominant and non-dominant scan were available. SD, standard deviation; RA, rheumatoid arthritis; early RA, RA <2 year disease duration; longstanding RA, RA>2 year disease duration; $M C P$ joint, metacarpophalangeal joint; HR-pQCT, high-resolution peripheral quantitative computed tomography; $B M D$, bone mineral density; Tot.BMD, total volumetric BMD; Tb.BMD, trabecular volumetric BMD; Ct.BMD, cortical volumetric BMD; Ct.TMD, cortical volumetric tissue mineral density; Tb.N, trabecular number; Tb.Th, trabecular thickness; Tb.Sp, trabecular separation; Tb.SpSD, intra-individual trabecular separation; Ct.Th, cortical thickness; Ct.Po, cortical porosity; Ct.Po.Dm, cortical porosity diameter. 
Table S6.4. HR-pQCT bone density, and micro-structure parameters in PIP joints of healthy subjects and patients with RA

\begin{tabular}{|c|c|c|c|c|c|c|c|c|c|}
\hline \multirow{3}{*}{ PIP joints } & \multirow{3}{*}{$\begin{array}{l}\text { Healthy } \\
\text { subjects } \\
n=57\end{array}$} & \multirow{3}{*}{$\begin{array}{c}\text { Patients } \\
\text { with RA } \\
n=122\end{array}$} & \multirow{3}{*}{$p$-value } & \multicolumn{6}{|c|}{ Patients with RA } \\
\hline & & & & $\begin{array}{l}\text { non- } \\
\text { dominant } \\
\text { hand }\end{array}$ & $\begin{array}{l}\text { Dominant } \\
\text { hand }\end{array}$ & $p$-value & early RA & $\begin{array}{c}\text { longstanding } \\
\text { RA }\end{array}$ & $p$-value \\
\hline & & & & $n=56^{*}$ & $n=56 *$ & & $n=34$ & $\mathrm{n}=88$ & \\
\hline \multicolumn{10}{|l|}{ Bone density parameters } \\
\hline Tot.BMD (mg HA/cm³) & $\begin{array}{l}518.8 \\
(45.6)\end{array}$ & $\begin{array}{l}467.2 \\
(72.8)\end{array}$ & $<0.001$ & $\begin{array}{l}467.0 \\
(71.3)\end{array}$ & $\begin{array}{l}467.6 \\
(75.7)\end{array}$ & 0.912 & $\begin{array}{l}466.6 \\
(38.7)\end{array}$ & $\begin{array}{l}467.4 \\
(82.5)\end{array}$ & 0.943 \\
\hline Tb.BMD (mg HA/cm³) & $\begin{array}{l}283.3 \\
(17.8)\end{array}$ & $\begin{array}{l}261.9 \\
(27.7)\end{array}$ & $<0.001$ & $\begin{array}{l}261.0 \\
(27.6)\end{array}$ & $\begin{array}{l}262.0 \\
(28.4)\end{array}$ & 0.640 & $\begin{array}{l}264.5 \\
(17.8)\end{array}$ & $\begin{array}{l}260.9 \\
(30.7)\end{array}$ & 0.424 \\
\hline Ct.BMD (mg HA/cm³) & $\begin{array}{l}837.3 \\
(41.8)\end{array}$ & $\begin{array}{l}813.6 \\
(51.2)\end{array}$ & 0.003 & $\begin{array}{l}816.5 \\
(51.9)\end{array}$ & $\begin{array}{l}812.3 \\
(47.5)\end{array}$ & 0.295 & $\begin{array}{l}807.6 \\
(65.5)\end{array}$ & $\begin{array}{l}815.9 \\
(44.7)\end{array}$ & 0.503 \\
\hline Ct.TMD (mg HA/ $\mathrm{cm}^{3}$ ) & $\begin{array}{l}950.8 \\
(30.3)\end{array}$ & $\begin{array}{l}928.3 \\
(43.6)\end{array}$ & $<0.001$ & $\begin{array}{l}929.5 \\
(42.8)\end{array}$ & $\begin{array}{l}929.5 \\
(42.8)\end{array}$ & 0.992 & $\begin{array}{l}928.0 \\
(49.8)\end{array}$ & $\begin{array}{l}928.5 \\
(41.3)\end{array}$ & 0.956 \\
\hline \multicolumn{10}{|c|}{ Bone micro-structure parameters } \\
\hline Tb.N $\left(\mathrm{mm}^{-1}\right)$ & $\begin{array}{c}1.83 \\
(0.20)\end{array}$ & $\begin{array}{c}1.72 \\
(0.23)\end{array}$ & 0.002 & $\begin{array}{c}1.70 \\
(0.24)\end{array}$ & $\begin{array}{c}1.71 \\
(0.22)\end{array}$ & 0.596 & $\begin{array}{c}1.72 \\
(0.20)\end{array}$ & $\begin{array}{c}1.72 \\
(0.24)\end{array}$ & 0.970 \\
\hline Tb.Th $(\mu \mathrm{m})$ & $\begin{array}{l}130.3 \\
(14.0)\end{array}$ & $\begin{array}{l}128.6 \\
(15.7)\end{array}$ & 0.481 & $\begin{array}{l}129.4 \\
(16.6)\end{array}$ & $\begin{array}{l}128.9 \\
(15.5)\end{array}$ & 0.729 & $\begin{array}{l}129.5 \\
(12.6)\end{array}$ & $\begin{array}{l}128.2 \\
(16.8)\end{array}$ & 0.684 \\
\hline Tb.Sp $(\mu \mathrm{m})$ & $\begin{array}{l}423.5 \\
(52.5)\end{array}$ & $\begin{array}{l}466.0 \\
(85.7)\end{array}$ & 0.001 & $\begin{array}{l}470.1 \\
(77.6)\end{array}$ & $\begin{array}{l}467.9 \\
(96.9)\end{array}$ & 0.809 & $\begin{array}{l}460.5 \\
(62.1)\end{array}$ & $\begin{array}{l}468.1 \\
(93.4)\end{array}$ & 0.663 \\
\hline Tb.SpSD $(\mu \mathrm{m})$ & $\begin{array}{c}346.5 \\
(115.3)\end{array}$ & $\begin{array}{c}401.5 \\
(140.4)\end{array}$ & 0.011 & $\begin{array}{c}405.9 \\
(134.3)\end{array}$ & $\begin{array}{c}401.0 \\
(151.4)\end{array}$ & 0.769 & $\begin{array}{c}372.9 \\
(100.4)\end{array}$ & $\begin{array}{c}412.6 \\
(152.1)\end{array}$ & 0.097 \\
\hline Ct.Th $(\mu \mathrm{m})$ & $\begin{array}{l}1,047.5 \\
(227.6)\end{array}$ & $\begin{array}{c}923.5 \\
(251.2)\end{array}$ & 0.002 & $\begin{array}{c}921.4 \\
(242.1)\end{array}$ & $\begin{array}{c}932.7 \\
(268.6)\end{array}$ & 0.612 & $\begin{array}{c}918.1 \\
(161.4)\end{array}$ & $\begin{array}{c}925.6 \\
(279.1)\end{array}$ & 0.855 \\
\hline Ct.Po (\%) & $\begin{array}{c}8.85 \\
(2.09)\end{array}$ & $\begin{array}{c}8.66 \\
(2.37)\end{array}$ & 0.600 & $\begin{array}{c}8.49 \\
(2.49)\end{array}$ & $\begin{array}{c}8.87 \\
(2.35)\end{array}$ & 0.077 & $\begin{array}{c}9.42 \\
(2.90)\end{array}$ & $\begin{array}{c}8.37 \\
(2.08)\end{array}$ & 0.060 \\
\hline Ct.Po.Dm $(\mu \mathrm{m})$ & $\begin{array}{c}278.7 \\
(147.1)\end{array}$ & $\begin{array}{l}229.9 \\
(67.7)\end{array}$ & 0.020 & $\begin{array}{l}229.2 \\
(64.5)\end{array}$ & $\begin{array}{l}229.3 \\
(62.8)\end{array}$ & 0.991 & $\begin{array}{l}221.6 \\
(20.8)\end{array}$ & $\begin{array}{l}233.2 \\
(78.5)\end{array}$ & 0.205 \\
\hline
\end{tabular}

Values are displayed as mean (SD). *Joints were included only if both the dominant and non-dominant scan were available. SD, standard deviation; early RA, RA $<2$ year disease duration; longstanding RA, RA $>2$ year disease duration; PIP joint, proximal interphalangeal joint; HR-pQCT, high-resolution peripheral quantitative computed tomography; $\mathrm{BMD}$, bone mineral density; Tot.BMD, total volumetric BMD; Tb.BMD, trabecular volumetric BMD; Ct.BMD, cortical volumetric BMD; Ct.TMD, cortical volumetric tissue mineral density; Tb.N, trabecular number; Tb.Th, trabecular thickness; Tb.Sp, trabecular separation; Tb.SpSD, intra-individual trabecular separation; Ct.Th, cortical thickness; Ct.Po, cortical porosity; Ct.Po.Dm, cortical porosity diameter. 

This chapter is embargoed at request

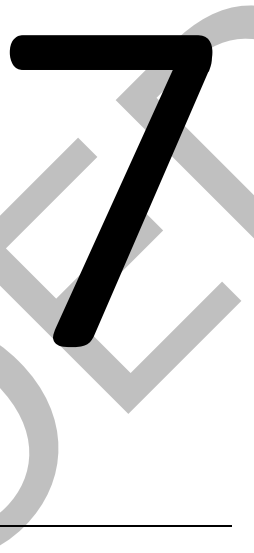

Prospective follow-up of cortical interruptions, bone density and micro-structure detected on high-resolution peripheral quantitative $\mathrm{CT}$ : a study in patients with RA and healthy subjects

M. Peters, J.P. van den Bergh, P. Geusens, A. Scharmga, D. Loeffen, R. Weijers, B. van Rietbergen, A. van Tubergen 


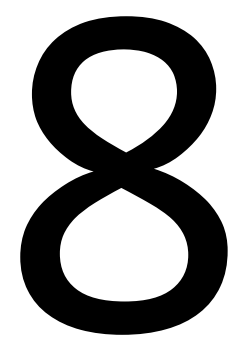

General discussion 

This thesis, described the development of an algorithm for automated image analysis for detection of small cortical interruptions in finger joints, which aimed at facilitating a more standardized and less time-consuming analysis of finger joints compared to visual scoring. When developing a new tool, it is important that it meets the requirements described in the Outcome Measures in Rheumatology (OMERACT, an independent initiative of health professionals interested in outcome measures in rheumatology): truth, discrimination and feasibility. ${ }^{1}$ By introducing this new automated algorithm, it is important to know whether this algorithm measures what is intended to measure (truth or validity), whether it discriminates between situations of interest (discriminatory value) and provides similar results on replication (reliability), and whether it is applicable in clinical practice in terms of time and interpretability (applicability).

We therefore, studied the reliability, validity, discriminatory value and applicability of HR-pQCT in the detection of small cortical interruptions, bone density and bone microstructure in finger joints of patients with RA and healthy subjects.

\section{DISCUSSION OF MAIN FINDINGS}

\section{Validity of HR-pQCT}

\section{Cortical interruptions}

When developing a new algorithm, an important question to answer is, "does it measure what is intended to measure", which is described in the OMERACT filter truth (validity). ${ }^{1}$ In Chapter 2, using visual scoring we showed that HR-pQCT is a sensitive (sensitivity = 81.6\%) imaging technique to detect cortical interruptions when using $\mu C T$ as gold standard. In addition, cortical interruptions that were present in at least 2 consecutive slices in 2 planes ( $2 \times 2$ slices) were frequently seen $(n=149)$ in MCP and PIP joints of anatomic specimens. However, since reliability of visual evaluation of HR-pQCT images between operators was fair to moderate ( $\mathrm{k} 0.37$ and ICC 0.55), an automated algorithm with (semi-automated method) and without (fully-automated method) manual correction of the outer cortical contours was developed, and both methods were compared to visual scoring (Chapter 3 ) and microCT ( $\mu \mathrm{CT}$ ) imaging (Chapter 4 ), as gold standards.

In Chapter 3, the median number of interruptions was significantly higher when using the algorithm compared to visual scoring (median per joint 14 versus $5, p<0.01$ ). We showed that only $28-30 \%$ of cortical interruptions $(\geq 0.246 \mathrm{~mm})$ detected by the fullyand semi-automated algorithm were identified with visual scoring, while $70-76 \%$ of the cortical interruptions detected visually were also detected by the fully- and semiautomated algorithm. Most interruptions detected with both the fully- and semiautomated algorithm, were visible as an interruption on a 2D grayscale image. However, these interruptions did not meet the pre-specified criteria of an interruption according 
to the SPECTRA definition that was used for visual scoring (i.e. an interruption visible in 2x2 HR-pQCT slices).

Differences between visual scoring and the semi-automated algorithm were mainly found for interruptions that were present within a region with a low density cortex or in case of (small) interruptions with a clear (dense) cortical lining. In a region of low cortical bone density it is often difficult to detect interruptions visually, while the algorithm can detect these interruptions more easily on binary images. However, due to partial volume effects (PVEs) interruption size may be overestimated. In contrast, small interruptions with a clear (dense) cortical lining, can easily be identified visually, but detection may be hampered when using the algorithm on binary images due to PVE.

Because the algorithm uses binary images to detect cortical interruptions, increasing the minimum cut-off diameter of interruptions, might be a solution to avoid false positive detection of cortical interruptions due to PVEs. Therefore, we compared semiautomated detection of cortical interruptions with different minimum diameters on HRpQCT to $\mu \mathrm{CT}$, with $\mu \mathrm{CT}$ as 3D gold standard because of its high resolution, in Chapter 4 . On the joint level, almost perfect agreement for the number of interruptions and interruption surface area was obtained for interruptions $\geq 0.41 \mathrm{~mm}$ (ICC 0.81 and 0.86 , respectively). The $\mathrm{PPV}_{\text {sd }}$ and sensitivity sd $_{\text {f }}$ for detection of a single interruption on HRpQCT with the same diameter on $\mu \mathrm{CT}$ was moderate to substantial $(66 \%$ and $49 \%$, respectively). The $P P V_{0.10 \mathrm{~mm}}$, with the use of a fixed $\mu \mathrm{CT}$ diameter of $\geq 0.10 \mathrm{~mm}$, was excellent (97\%).

The PPV $\mathrm{S}_{\text {sd }}$ and sensitivity sd $_{\text {of }}$ visual scoring was higher compared to the semi-automated algorithm (interruptions with diameter $\geq 0.41 \mathrm{~mm}$ ) (PPV sd $_{\text {and }}$ ansitivity sd $_{\text {s }}: 81.6 \%$ and $81.6 \%$ versus $66.3 \%$ and $49.4 \%$ ). However, when considering the presence of interruptions on $\mu \mathrm{CT}$ not taking into account the diameter, PPV was higher for the semiautomated algorithm (PPV $0.10 \mathrm{~mm}$ 97\%). In addition, for calculation of the PPV and sensitivity of the semi-automated algorithm, interruptions were considered matching when they were on exactly the same location. For visual scoring, however, interruptions were considered matching if an interruption was scored in the same joint quadrant. Hence, this probably led to an overestimation of the PPV and sensitivity of the visual scoring.

The validity of our algorithm was further explored in Chapter 6. We compared the number, surface area and volume of interruptions detected on HR-pQCT images with clinical standards (CR and MRI) in 38 healthy females and 39 female patients with RA of the MOSA-Hand cohort. We found that structural damage on CR and MRI was significantly associated with the number, surface area and volume of interruptions (Prevalence Ratio's (PRs) ranging from 1.3 to 2.0). For example, every erosion on MRI was associated with a $32 \%$ increase of the number of interruptions, $84 \%$ increase of interruption surface area and 92\% increase of interruption volume. Markers of inflammation on MRI (BME and synovitis) were also significantly associated with the number, surface area and volume of interruptions (PRs ranging from 2.3 to 7.7). 
Our findings were in line with previous studies, showing that the presence of BME on MRI is associated with cortical interruptions (i.e. erosions) and progression of structural bone damage on radiographs. ${ }^{2-4}$ The study of Lee et al. compared structural damage on $\mathrm{CR}$ and $\mathrm{MRI}$, and BME on MRI with erosions on HR-pQCT. Strong correlations were found between structural damage on CR and erosions on HR-pQCT (Spearman $r$ ranging from 0.66 to $0.87, p<0.05$ ), and between BME MRI and erosions on HR-pQCT (Spearman $r$ ranging from 0.46 to $0.62, p<0.05)$, and erosions on $M R I$ and erosions on HR-pQCT (Spearman $r$ ranging from 0.86 to $0.88, p<0.05) .{ }^{5}$ The study of Albrecht et al. compared erosions on MRI to HR-pQCT, with HR-pQCT as reference method. Sensitivity and PPV were $94 \%$ and $97 \%$ for MRI to HR-pQCT, respectively. ${ }^{6}$

In summary, we validated a semi-automated algorithm compared to visual scoring on HR-pQCT and to $\mu C T$ (as 3D gold standard), and clinical standards CR and MRI. We showed that interruptions detected with the automated algorithm were also detected visually and on $\mu C T$, and structural damage on $C R$ and $M R I$ were associated with interruptions detected by the automated algorithm on HR-pQCT. Hence, we showed that the automated algorithm measures what is intended to measure

\section{Reliability of HR-pQCT}

When investigating a newly developed algorithm, an important question to answer is, "does it provide similar results on replication" (reliability), which is described in the OMERACT filter discrimination. ${ }^{1}$

\section{Cortical interruptions}

At the start of this thesis, the reliability of visual detection of small cortical interruptions on HR-pQCT images was unknown. Therefore, in Chapter 2, we evaluated the intra- and inter-reader reliability of visually scored small cortical interruptions in anatomic specimens of finger joints. The intra-reader reliability was moderate to substantial (ICC 0.61 and $\mathrm{k} 0.52$ ) and the inter-reader reliability was fair to moderate (ICC 0.55 and к 0.37).

In Chapter 3 and 5, we aimed to improve reliability of cortical interruption detection by introducing an automated algorithm to detect cortical interruptions $\geq 0.246 \mathrm{~mm}$ (Chapter 3) and $\geq 0.41 \mathrm{~mm}$ (Chapter 5) on HR-pQCT images of MCP joints from patients with RA and healthy subjects from the MOSA-Hand cohort. Also, the additional value of manual correction of automatically generated outer cortical contours was investigated (semi-automated approach) in Chapter 3. Almost perfect reliability for the number of cortical interruptions (ICC 0.97 and 0.94), interruption surface area (ICC 0.98 and 0.70 ) and volume (ICC 0.91) was observed when applying both the fully- and semi-automated algorithm. Although the reliability of the fully- and semi-automated procedure was comparable, we believe that the semi-automated procedure is the preferred method, because manual corrections of the outer cortical contours may be necessary in case of 
large cortical interruptions and considerable motion artefacts in order to correct for auto-contouring inaccuracies.

In Chapter 5, we investigated the reproducibility and precision errors of cortical interruption detection when using repeated scans with complete in-between repositioning in early arthritis patients. The reproducibility of the extended algorithm for the number, surface area and volume of cortical interruptions was excellent (all ICCS $\geq 0.82$ ), especially for the interruption volume (ICC 0.99). The calculated LSC SD $_{\text {Sere }} 4.2$ for the number of interruptions, $5.8 \mathrm{~mm}^{2}$ for interruption surface area, and $3.2 \mathrm{~mm}^{3}$ for interruption volume. Although a high reproducibility was obtained, the $\mathrm{LSC}_{\mathrm{SD}}$ values were substantial compared to the absolute mean values detected.

When comparing the reliability of the semi-automated algorithm (Chapter 3 and 5) to visual scoring (Chapter 2), we found that the inter-operator reliability was indeed better when using the semi-automated algorithm for the number of interruptions (ICC $\geq 0.94$ versus 0.55 , respectively).

In contrast to the findings in this thesis, others reported reliability of visual scoring of cortical interruptions for large erosions only. ${ }^{7-9}$ Semi-quantitative grading of these erosions showed a good intra- and inter-reader reliability $(\kappa \geq 0.75$ and ICC $\geq 0.89)$. ${ }^{7,8}$ Moreover, Barnabe et al. reported a moderate inter-reader reliability between 11 readers for the presence or absence of erosions per quadrant ( $\mathrm{k} 0.52)$, but when restricted to experienced readers (>5 years of experience) $\mathrm{k}$ was $0.75 .^{9}$ In addition, reliability of the size of single erosions was reported using 4 distance measurements (i.e. width and depth in the axial plane, and width and depth in the perpendicular plane). The ICCs for all 11 readers ranged from fair to almost perfect (range 0.21-0.87, perpendicular width and axial depth, respectively), but when restricted to experienced readers, ICCS were $>0.90 .^{9}$

Lastly, Töpfer et al. developed a 3D automated volume determination of erosions and reported the intra- and inter-operator reliability of single erosions. ${ }^{10}$ The $\mathrm{LSC}_{\mathrm{SD}}$ values were lower compared to our $L_{S C}$ SD values LSC $_{S D} 3.2 \mathrm{~mm}^{3}$ versus $1.4 \mathrm{~mm}^{3}$ and $2.1 \mathrm{~mm}^{3}$, respectively), but the study from Töpfer et al. differed in several aspects from ours. In that study, only erosions, and the volume of a selection of erosions were analysed by two operators twice on a single scan. In contrast, we used data from repeated scans and included all interruptions, irrespective whether they were detected on the first scan but not on the second scan and vice versa. This approach will result in higher reproducibility errors.

In general, a higher reliability was obtained for the semi-automated algorithm compared to visual scoring for the number and size of interruptions, but comparison to other studies is difficult because we also included small interruptions in contrast to the previous studies. The $\mathrm{LSC}_{\mathrm{SD}}$ values and precision errors in previous studies were, however, in the same order of magnitude. 


\section{$\checkmark B M D$ and bone micro-structure}

At the start of this thesis, the intra- and inter-operator reliability and intra-operator reproducibility of HR-pQCT derived $\mathrm{VBMD}$ and bone micro-structural parameters of finger joints in arthritis patients were unknown. Therefore, in Chapter 5, the intra- and inter-operator reliability and intra-operator reproducibility of all VBMD and bone microstructural parameters were studied using semi-automated assessment. Both intra- and inter-operator reliability (ICCs $>0.99$ ) as well as the intra-operator reproducibility were excellent for $\mathrm{VBMD}$ and bone micro-structural parameters (ICCs $\geq 0.97$ ) and good for the cortical porosity diameter (Ct.Po.Dm, ICC 0.84). The precision errors $\left(\mathrm{CV}_{\mathrm{RMS}}\right)$ were generally below $2 \%$ for the vBMD parameters (except for Tb.BMD), below $5 \%$ for the trabecular bone parameters (except for Tb.SpSD), and below $10 \%$ for the cortical bone parameters. The least significant changes $\left(\mathrm{LSC}_{\%}\right)$ were ranging from $1.0 \%$ (Ct.TMD) to $24.1 \%$ (Ct.Po).

To the best of our knowledge, we were the first to study the intra- and inter-operator reliability of HR-pQCT assessment of VBMD and bone micro-structural parameters in finger joints. The precision errors of repeated scans in our study were comparable to those reported by others, except for the Ct.Po parameter, which was substantially lower in our study compared to Feehan et al. (8.7\% versus $27.7 \%) .{ }^{11}$ In addition, the precision errors of finger joint HR-pQCT scans were comparable to those observed in HR-pQCT scans of the distal radius and tibia. ${ }^{12}$

In summary, based on the findings in this thesis, it is reliable to use a semi-automated algorithm for detection of cortical interruptions, and assessment of VBMD and bone micro-structural parameters on HR-pQCT images of finger joints.

\section{Discriminatory value of $\mathrm{HR}-\mathrm{pQCT}$}

When investigating a newly developed algorithm, important questions to answer are, "whether it discriminates between situations of interest" (discriminatory value), which is described in the OMERACT filter discrimination. ${ }^{1}$

\section{Cortical interruptions}

In Chapter 6, we therefore tested the semi-automated algorithm in a cross-sectional clinical study in order to determine its potential value in discriminating patients with RA from healthy subjects, preferably early in the disease course. We found more and larger cortical interruptions in more joints in patients with RA compared to healthy subjects (means, 4.3 and $27.0 \mathrm{~mm}^{3}$ versus 1.5 and $1.5 \mathrm{~mm}^{3}$ per MCP joint, respectively), also after correction for within-subject correlation and age. More and larger interruptions were already found in early RA patients (disease duration <2 years) compared to healthy subjects. The presence of RA was associated with more and larger interruptions even after adjustment of findings on CR and MRI. This suggests that HR-pQCT is of added 
value in the detection of small cortical interruptions and in the distinction of patients with RA and healthy subjects.

This is in line with a previous study using visual scoring on binary images, where more very small cortical interruptions (with a diameter $\geq 0.082 \mathrm{~mm}$ ) were found in patients with RA compared to healthy subjects (112.9 versus 75.2$).{ }^{13}$ Other studies using visual analysis showed already that more pathological interruptions in patients with RA could be found compared to healthy subjects, ${ }^{7,8,14,15}$ and interruptions $>1.9 \mathrm{~mm}$ were considered highly specific for patients with RA. ${ }^{8}$

We further explored the discriminatory value of cortical interruptions in Chapter 7. Here, we prospectively studied the change in the number, surface area and volume of cortical interruptions in patients with RA (treated with synthetic disease modifying antirheumatic drugs (sDMARDs) and/or biologic DMARDs (bDMARDs)) versus healthy subjects after a one-year follow-up period using our semi-automated algorithm. We showed that the mean in cortical interruption parameters did not change at the group level in both patients with RA and healthy subjects, except for a significant increase in the number of interruptions in PIP joints of patients with RA. No difference in mean change of cortical interruptions was found between patients treated with sDMARDs compared with patients on bDMARDs. In contrast, at the individual joint level, joints could be show bone repair and loss, both mainly in patients with RA.

This is in line with the study of Töpfer et al. who also reported no change in the mean erosion volume between baseline and one year follow-up in patients with RA. ${ }^{16}$ Additionally, they identified multiple erosions that significantly increased or decreased in volume, as we found. The effect of bDMARD treatment on repair of interruptions detected on HR-pQCT has been described earlier. ${ }^{17-19}$ A decrease in erosion size was observed in patients treated with bDMARDs compared with an increase in erosion size in patients treated with sDMARDs after 1 year, ${ }^{18}$ and after 3 months. ${ }^{17}$ In another study in patients on bDMARDs, a decrease in erosion size was found after 1 year, but also nine new erosions were detected. ${ }^{19}$ In this study, no comparison was made to an SDMARD treatment group. Future research should further explore which patients show progressive bone damage and show bone repair processes.

\section{$\checkmark B M D$ and bone micro-structure}

We investigated the $V B M D$ and bone micro-structure in Chapter 6 in patients with RA compared to healthy subjects. We found that the $\mathrm{VBMD}$ and bone micro-structural parameters were impaired in patients with RA (a lower VBMD in both the trabecular and cortical region, fewer trabeculae (Tb.N lower, Tb.Sp and Tb.SpSD higher), and a thinner cortex). This is in agreement with previous studies investigating VBMD and bone microstructure parameters in patients with RA compared to healthy subjects using HR$\mathrm{pQCT},{ }^{14,20}$ although the study from Fouque-Aubert et al. reported thinner trabeculae rather than fewer trabeculae, as was found in our study. ${ }^{14}$ This difference in finding might be explained by the longer scan region in our study compared to the study of 
Fouque-Aubert et al. (3 stacks $(27.06 \mathrm{~mm})$ versus 1 stack $(9.02 \mathrm{~mm})$ ), because more distally from the joint fewer trabeculae are present. ${ }^{14}$

We were the first that also evaluated Ct.TMD and Ct.Po in finger joints of patients with RA in comparison to healthy subjects. We found that the Ct.TMD was significantly lower in patients with RA, meaning that the decrease in cortical VBMD (Ct.BMD) is not only caused by the loss of cortical bone (thinning and increase in porosity of the cortical bone), but also by a decrease of the density of cortical bone tissue. Ct.Po was comparable between patients with RA and healthy subjects. It should be noted that the Ct.Po measurement only considers intra-cortical pores, hence cortical interruptions are excluded.

We further explored the discriminatory value in Chapter 7. Here, we prospectively studied the change in VBMD and bone micro-structural parameters in patients with RA (treated with ssDMARDs and/or bDMARDs) versus healthy subjects after a one-year follow-up period. Volumetric BMD decreased significantly more in patients with RA compared to healthy subjects $\left(-4.4\right.$ and -5.8 versus -1.1 and $-2.6 \mathrm{mgHA} / \mathrm{cm}^{3}$ in $\mathrm{MCP}$ and PIP joints, respectively). The decrease in trabecular thickness was significantly more in patients with RA compared to healthy subjects in PIP joints. In addition, the proportion of joints with loss of VBMD beyond LSC was significantly higher in patients with RA than in healthy subjects $(26.7 \%$ versus $12.9 \%$, respectively, OR $2.6(95 \% \mathrm{Cl} 1.4-5.0))$. The proportion of joints with loss of VBMD beyond LSC was significantly higher in patients treated with sDMARDs compared with patients treated with bDMARDs (31.3\% versus $17.2 \%$, respectively, OR $2.7(95 \% \mathrm{Cl} 1.4-5.3))$.

In the study of Töpfer et al. no change in mean VBMD between baseline and one year follow-up in patients with RA was found. However, VBMD was only analysed in the vicinity of erosions. ${ }^{16}$ In the study of Shimizu et al., no change in mean VBMD was observed in patients treated with bDMARDs compared to sDMARDs, but the follow-up period was only 3 months. ${ }^{17}$ Another study showed that patients additionally treated with denosumab had an increase in $\mathrm{VBMD}$, but patients additionally treated with alendronate had a decrease in VBMD. No study longitudinally evaluated the bone microstructural parameters in finger joints of patients with RA.

\section{Applicability of HR-pQCT}

When investigating a newly developed algorithm, an important question to answer is, "Can the measure be applied easily, given constraints of time, money, and interpretability?" (applicability), which is described in the OMERACT filter feasibility. ${ }^{1}$

At the start of this thesis no studies on the feasibility of HR-pQCT use had been performed. HR-pQCT scanning time in our study was approximately 30 minutes, when using 330 (9 minutes) and 220 slices (6 minutes) for the second and third MCP and PIP joints of both hands respectively. Compared to MRI, which takes up to one hour for both hands, it is less time consuming, but CR of the hands takes no more than a few seconds. Furthermore, studies on the time to evaluate HR-pQCT images were scarce. A study of 
Srikhum et al. reported visual scoring time using semi-quantitative grading of erosions for analysis of one MCP joint (330 slices), and found a median scoring time of 2 minutes (range 1.20-5.30 minutes). ${ }^{7}$ Hence, visual semi-quantitative grading might be feasible for clinical practice, but only large erosions could be detected using this method, thereby limiting its added value over CR and MRI. In a previous study from our study group, we estimated the average scoring time for visual scoring incorporating all cortical interruptions. An average of 9.2 minutes (range 1-38 minutes) for scoring one MCP or PIP joint was needed and we concluded that visual scoring of small cortical interruptions is therefore not feasible to apply in clinical practice. ${ }^{21}$

Using our semi-automated algorithm, we may decrease the scoring time per joint, but we did not investigate the average time necessary for manual correction in the outer cortical contours. The number of manual corrections depends on the presence and severity of motion artefacts and the number of large cortical interruptions (interruption surface area $>1 \mathrm{~mm}^{2}$ ) within the joint. However, these corrections are also necessary for the assessment of the $\mathrm{VBMD}$ and bone micro-structural parameters. Hence, the correction of the contours is not a limitation of the algorithm, and after correction of the contours, the number and size of cortical interruptions, vBMD and bone micro-structural parameters can be assessed automatically.

\section{Limitations}

The work presented in this thesis has several limitations.

First, in Chapter 3, only a small number of finger joint scans was included (10 scans). However, a large number of cortical interruptions (199 in total) was detected in these joints, which still provided reliable estimates for the proportion of matching interruptions.

Second, in Chapters 2 and 4 we used a sample of anatomic specimens of elderly women. These specimens had a low bone mineralization (average $\mathrm{vBMD}=245 \mathrm{mgHA} / \mathrm{cm}^{3}$ compared to $>300 \mathrm{mgHA} / \mathrm{cm}^{3}$ in healthy subjects ${ }^{22,23}$ ). Due to thin and low mineralized cortices, false positive detection of cortical interruptions might have occurred. This may have affected the number of detected interruptions, hence the reliability and validity of detected interruptions. Therefore, our findings regarding validity and reliability may not be extrapolated to young, healthy subjects and patients with RA.

Third, PVEs are inherent to imaging, hence also to HR-pQCT. However, PVEs can especially be important in binary images. Due to a reduced cortical bone mineral density in patients with RA, thin and low mineralized cortices can falsely be identified as cortical interruptions. However, we showed that most interruptions on HR-pQCT were also detected on $\mu \mathrm{CT}$ imaging.

Fourth, motion artefacts are a common finding in HR-pQCT imaging. ${ }^{24}$ In Chapter 5, 23 stacks of 126 stacks (18.3\%) were of poor quality due to motion artefacts. One scan of a finger joint comprises of multiple stacks, if one stack was of poor quality we considered the whole scan as poor quality. In Chapters 6 and 7, scans were repeated 
once if the first scan was of insufficient quality, but we still found poor quality scans in $8.2 \%$ of all scans in the MOSA-Hand cohort. The number of poor quality scans was lower compared to radius scanning where motion artefacts occur in approximately $30 \%$ of the scans. ${ }^{25}$ This might be because of the better fixation in the scan holder of the hand compared to the radius. Therefore, in research using HR-pQCT imaging, one need to bear in mind that patient motion is regularly observed and should be considered when performing a power and radiation exposure calculation.

Fifth, this thesis focused on cortical interruptions, VBMD and bone micro-structure, but RA is also characterized by joint space narrowing which was not studied in this thesis. An algorithm for semi-automated detection of the mean joint space width and volume has already been developed and showed promising results. ${ }^{26}$ Therefore, HR-pQCT might also be used for joint space width measurements in patients with RA.

Sixth, our studies were performed using the HR-pQCT1. Meanwhile, the HR-pQCT2 has been introduced, which has a higher spatial resolution ( $95 \mu \mathrm{m}$ versus $130 \mu \mathrm{m}$ ) and smaller voxel size $(61 \mu \mathrm{m}$ versus $82 \mu \mathrm{m})$. This higher resolution will enable better distinction between bone and non-bone. Additionally, the parameters (cortical mask thickness, minimum interruption diameter and minimum trabecular void volume) used in the semi-automated algorithm should be adapted to obtain similar values. The cortical mask should be increased to 5 voxels to obtain a similar cortical mask thickness ( 5 voxels of $61 \mu \mathrm{m}=0.305 \mathrm{~mm}$ versus $0.328 \mathrm{~mm}$ ). In addition, the minimum interruption diameter is smaller when still applying 2 dilation steps $(\geq 0.305 \mathrm{~mm}$ versus $\geq 0.410 \mathrm{~mm}$, respectively). One may consider to increase the number of dilation steps to 3 , hence interruptions $\geq 0.427 \mathrm{~mm}$ would be detected, which is closer to the minimum interruption diameter of $\geq 0.410 \mathrm{~mm}$ of the semi-automated algorithm on HR-pQCT 1 . Lastly, the detection of the trabecular void volume may be reconsidered. Now, voids with a diameter of at least $0.728 \mathrm{~mm}$ (i.e. 9 voxels of $82 \mu \mathrm{m}$ ) are considered, because this is larger than the trabecular separation which is regularly $(<5 \%)$ observed in MCP joints of healthy subjects. Using the HR-pQCT 2, the new void could be decreased to $0.671 \mathrm{~mm}$ (i.e. 11 voxels of $61 \mu \mathrm{m}$ ) or increased to $\geq 0.793 \mathrm{~mm}$ (i.e. 13 voxels $61 \mu \mathrm{m}$ ). Hence, studies should be performed that compare the validity, reliability and the discriminatory value of the interruptions that are detected with the different cut-off values.

Lastly, the automated algorithm developed in this thesis has several limitations that should be noted. First, the cortical mask was set to a constant thickness, because this would avoid any inaccuracies in the detection of the endosteal cortical bone contour. A disadvantage of this approach is that in relatively thick cortical regions, that are located more distal from the joint, cortical interruptions may be detected which are not connected to the endosteal cortical bone surface and in fact are not cortical interruptions. Second, the trabecular void volume underlying the cortical interruption is limited to a depth of $4 \mathrm{~mm}$ in MCP joints and $2 \mathrm{~mm}$ in PIP joints. This means that the algorithm underestimates the volume of interruptions with a depth greater than these cut-offs (i.e. volume $>134 \mathrm{~mm}^{3}$ and $16.8 \mathrm{~mm}^{3}$, respectively). Hence, the 3D volume of 
very large interruptions detected by our algorithm may be different compared to other approaches. However, such large interruptions are not the primary focus of research with HR-pQCT, because they can also be detected by other imaging techniques with lower resolution (e.g. CR and MRI). The strength of HR-pQCT lies mainly in detection of "small" interruptions, i.e. $<10 \mathrm{~mm}^{3}$, which are regularly missed with MRI. ${ }^{6}$ Third, the automated algorithm still requires manual correction of the outer cortical contours in case of large cortical interruptions and motion artefacts, which can make the analysis time consuming. This correction is also advised by the manufacturer as part of the standard evaluation protocol for assessment of $\mathrm{VBMD}$ and bone micro-structural parameters. Hence, no extra manual correction is necessary for the assessment of cortical interruptions. After the correction, analysis can be performed for cortical interruption-, vBMD-, and bone micro-structural parameters. In addition, these contours can be used for the joint space width algorithm. ${ }^{26}$

\section{FUTURE PERSPECTIVES}

Based on the findings in this thesis, HR-pQCT imaging is a potential new imaging method for diagnosis and monitoring of patients with RA. Future studies may focus on further automation and optimization of the algorithm. Limitations of the algorithm such as the constant thickness of the cortical mask, PVE and the requirement of manual correction of the outer cortical contours have to be further optimized.

For example, instead of choosing a constant thickness of the cortical mask, one may consider the use of the endosteal contouring as described by Burghardt et al., ${ }^{27}$ as this might lower the detection of cortical interruptions that are not connected to the endosteal cortical bone surface. However, this might be less accurate in cases where a new cortex is formed in the joint.

Further automation can be performed by optimizing the outer cortical contour. This might be resolved by enhanced filtering of motion artefacts or by making a standard "normal" atlas based finger joint (i.e. a mould) that can be used as a reference finger contour. This has already been successfully applied in tibiofemoral joint and intervertebral discs. ${ }^{28,29}$

Next, the influence of PVEs may be reduced by using the second generation HR-pQCT 2. The HR-pQCT 2 has a higher spatial resolution compared to the HR-pQCT 1 (95 $\mu \mathrm{m}$ versus $130 \mu \mathrm{m}$, respectively). Thus, HR-pQCT 2 might be able to detect these thin structures, which may reduce the number of interruptions detected falsely as a result of PVE.

Next, it would be interesting to use HR-pQCT imaging with the semi-automated algorithm in clinical trials to evaluate and monitor the effects of therapy. Additionally, the joint space width could be additionally evaluated, since RA is also characterized by joint space narrowing which was not studied in this thesis. An algorithm for automated detection of the mean joint space width and volume has already been developed and 
showed promising results. ${ }^{26}$ In addition, given the prospects of high resolution imaging of finger joints, this field of research may be expanded to other bone affecting rheumatic diseases, such as psoriatic arthritis, osteoarthritis and gout.

\section{OVERALL CONCLUSION}

This thesis showed that semi-automated analysis of HR-pQCT images is a valid, reliable and feasible tool, for detection of cortical interruptions with a diameter $\geq 0.41 \mathrm{~mm}$, and for assessment of VBMD and bone micro-structural parameters in finger joints. HR-pQCT imaging can discriminate between patients with RA and healthy subjects both crosssectionally and longitudinally, and may attribute in the evaluation of peri-articular bone involvement in finger joints of patients with RA.

We developed a semi-automated algorithm for detection of small cortical interruptions and showed that the algorithm was more reliable compared to visual scoring. The algorithm was validated for cortical interruption detection compared to visual scoring of HR-pQCT images, $\mu C T, C R$ and MRI. Structural damage and inflammatory features on CR and MRI were associated with more and larger cortical interruptions on HR-pQCT. More and larger interruptions were found in patients with RA compared to healthy subjects independent of findings on CR or MRI. When applying the algorithm, significant progression as well as significant repair of cortical interruptions was found in individual joints of patients with RA after one year follow-up. Additionally we showed that VBMD and bone micro-structural parameters are impaired in patients with RA already early in the disease course compared to healthy subjects. Longitudinal evaluation also showed that both VBMD and bone micro-structural parameters decreased significantly more in patients with RA compared to healthy subjects. Patients treated with bDMARDs had less decrease of vBMD compared to patients treated with sDMARDs. Altogether, this thesis shows that HR-pQCT holds a great promise in the investigation of (early) bone damage in finger joints of arthritis patients and may prove to be of additional value to currently available imaging techniques, not only for detecting cortical interruptions but also by quantifying $\mathrm{VBMD}$ and micro-structure over time. 


\section{REFERENCES}

1. Boers M, Brooks P, Strand CV, Tugwell P. The OMERACT filter for Outcome Measures in Rheumatology. J Rheumatol. 1998;25(2):198-9.

2. Gandjbakhch F, Foltz V, Mallet A, Bourgeois P, Fautrel B. Bone marrow oedema predicts structural progression in a 1-year follow-up of 85 patients with RA in remission or with low disease activity with low-field MRI. Ann Rheum Dis. 2011;70(12):2159-62.

3. Hetland ML, Ejbjerg B, Horslev-Petersen K, Jacobsen S, Vestergaard A, Jurik AG, et al. MRI bone oedema is the strongest predictor of subsequent radiographic progression in early rheumatoid arthritis. Results from a 2-year randomised controlled trial (CIMESTRA). Ann Rheum Dis. 2009;68(3):384-90.

4. Peterfy C, Strand V, Tian L, Ostergaard M, Lu Y, DiCarlo J, et al. Short-term changes on MRI predict longterm changes on radiography in rheumatoid arthritis: an analysis by an OMERACT Task Force of pooled data from four randomised controlled trials. Ann Rheum Dis. 2017;76(6):992-7.

5. Lee CH, Srikhum W, Burghardt AJ, Virayavanich W, Imboden JB, Link TM, et al. Correlation of structural abnormalities of the wrist and metacarpophalangeal joints evaluated by high-resolution peripheral quantitative computed tomography, 3 Tesla magnetic resonance imaging and conventional radiographs in rheumatoid arthritis. Int J Rheum Dis. 2015;18(6):628-39.

6. Albrecht A, Finzel S, Englbrecht M, Rech J, Hueber A, Schlechtweg P, et al. The structural basis of MRI bone erosions: an assessment by microCT. Ann Rheum Dis. 2013;72(8):1351-7.

7. Srikhum W, Virayavanich W, Burghardt AJ, Yu A, Link TM, Imboden JB, et al. Quantitative and semiquantitative bone erosion assessment on high-resolution peripheral quantitative computed tomography in rheumatoid arthritis. J Rheumatol. 2013;40(4):408-16.

8. Stach CM, Bauerle M, Englbrecht M, Kronke G, Engelke K, Manger B, et al. Periarticular bone structure in rheumatoid arthritis patients and healthy individuals assessed by high-resolution computed tomography. Arthritis Rheum. 2010;62(2):330-9.

9. Barnabe C, Toepfer D, Marotte H, Hauge EM, Scharmga A, Kocijan R, et al. Definition for Rheumatoid Arthritis Erosions Imaged with High Resolution Peripheral Quantitative Computed Tomography and Interreader Reliability for Detection and Measurement. J Rheumatol. 2016;43(10):1935-40.

10. Topfer D, Finzel S, Museyko O, Schett G, Engelke K. Segmentation and quantification of bone erosions in high-resolution peripheral quantitative computed tomography datasets of the metacarpophalangeal joints of patients with rheumatoid arthritis. Rheumatology. 2014;53(1):65-71.

11. Feehan. A customized protocol to assess bone quality in the metacarpal head, a HRpQCT study.

12. Boutroy S, Bouxsein ML, Munoz F, Delmas PD. In vivo assessment of trabecular bone microarchitecture by high-resolution peripheral quantitative computed tomography. J Clin Endocrinol Metab. 2005; 90(12):6508-15.

13. Werner D, Simon D, Englbrecht M, Stemmler F, Simon C, Berlin A, et al. Rheumatoid arthritis is characterized by early changes of the cortical micro-channel (CoMiC) system in the bare area of the joints. Arthritis Rheum. 2017;69(8):1580-7.

14. Fouque-Aubert A, Boutroy S, Marotte H, Vilayphiou N, Bacchetta J, Miossec P, et al. Assessment of hand bone loss in rheumatoid arthritis by high-resolution peripheral quantitative CT. Ann Rheum Dis. 2010;69(9):1671-6.

15. Barnabe C, Szabo E, Martin L, Boyd SK, Barr SG. Quantification of small joint space width, periarticular bone microstructure and erosions using high-resolution peripheral quantitative computed tomography in rheumatoid arthritis. Clin Exp Rheumatol. 2013;31(2):243-50.

16. Topfer D, Gerner B, Finzel S, Kraus S, Museyko O, Schett G, et al. Automated three-dimensional registration of high-resolution peripheral quantitative computed tomography data to quantify size and shape changes of arthritic bone erosions. Rheumatology. 2015;54(12):2171-80.

17. Shimizu T, Choi HJ, Heilmeier U, Tanaka M, Burghardt AJ, Gong J, et al. Assessment of 3-month changes in bone microstructure under anti-TNFalpha therapy in patients with rheumatoid arthritis using highresolution peripheral quantitative computed tomography (HR-pQCT). Arthritis Res Ther. 2017;19(1):222.

18. Finzel S, Rech J, Schmidt S, Engelke K, Englbrecht M, Stach C, et al. Repair of bone erosions in rheumatoid arthritis treated with tumour necrosis factor inhibitors is based on bone apposition at the base of the erosion. Ann Rheum Dis. 2011;70(9):1587-93. 
19. Finzel S, Rech J, Schmidt S, Engelke K, Englbrecht M, Schett G. Interleukin-6 receptor blockade induces limited repair of bone erosions in rheumatoid arthritis: a micro CT study. Ann Rheum Dis. 2013;72(3):396-400.

20. Yang H, Yu A, Burghardt AJ, Virayavanich W, Link TM, Imboden JB, et al. Quantitative characterization of metacarpal and radial bone in rheumatoid arthritis using high resolution- peripheral quantitative computed tomography. Int J Rheum Dis. 2017;20(3):353-62.

21. Scharmga A. High-resolution peripheral quantitative computed tomography in patients with rheumatoid arthritis: truth, discrimination and feasibility. Thesis UM 2017.

22. Scharmga A, Peters M, van Tubergen A, van den Bergh J, de Jong J, Loeffen D, et al. Visual detection of cortical breaks in hand joints: reliability and validity of high-resolution peripheral quantitative $\mathrm{CT}$ compared to microCT. BMC Musculoskelet Disord. 2016;17(1):271.

23. Peters $M$, Scharmga A, van Tubergen A, Arts J, Loeffen $D$, Weijers $R$, et al. The Reliability of a Semiautomated Algorithm for Detection of Cortical Interruptions in Finger Joints on High Resolution CT Compared to MicroCT. Calcif Tissue Int. 2017;101(2):132-40.

24. Pialat JB, Burghardt AJ, Sode M, Link TM, Majumdar S. Visual grading of motion induced image degradation in high resolution peripheral computed tomography: impact of image quality on measures of bone density and micro-architecture. Bone. 2012;50(1):111-8.

25. Geusens P, Chapurlat R, Schett G, Ghasem-Zadeh A, Seeman E, de Jong J, et al. High-resolution in vivo imaging of bone and joints: a window to microarchitecture. Nat Rev Rheumatol. 2014;10(5):304-13.

26. Burghardt AJ, Lee CH, Kuo D, Majumdar S, Imboden JB, Link TM, et al. Quantitative in vivo HR-pQCT imaging of 3D wrist and metacarpophalangeal joint space width in rheumatoid arthritis. Ann Biomed Eng. 2013;41(12):2553-64.

27. Burghardt AJ, Buie HR, Laib A, Majumdar S, Boyd SK. Reproducibility of direct quantitative measures of cortical bone microarchitecture of the distal radius and tibia by HR-pQCT. Bone. 2010;47(3):519-28.

28. Michopoulou SK, Costaridou L, Panagiotopoulos E, Speller R, Panayiotakis G, Todd-Pokropek A. Atlasbased segmentation of degenerated lumbar intervertebral discs from MR images of the spine. IEEE Trans Biomed Eng. 2009;56(9):2225-31.

29. Brehler M, Thawait G, Shyr W, Ramsay J, Siewerdsen JH, Zbijewski W. Atlas-based automatic measurements of the morphology of the tibiofemoral joint. Proc SPIE Int Soc Opt Eng. 2017;10137. 


\section{ADDENDUM}

Valorisation

Summary

Nederlandse samenvatting

Dankwoord

Curriculum Vitae

Publications 



\section{VALORISATION}

Valorisation of research is defined as "the process of creating value from knowledge, by making this knowledge available and suitable for economic and social exploitation and to translate this knowledge into products, services, processes, and new business". Or, how can society benefit from the knowledge acquired with research? In this chapter I aimed to describe the clinical relevance of this thesis and relevance of future research of highresolution peripheral quantitative computed tomography (HR-pQCT) imaging.

During my research I have only seen a fraction of patients suffering from rheumatoid arthritis (RA). Some of the patients were not able to get in or out of the bed or dress themselves. These limitations will probably be there for the rest of their lives. Since there is no cure for RA, the treatment of the patients is directed at achieving symptom improvement, a low disease activity state or remission, and to inhibit damage to bone, ligaments and tendons. However, despite achieving clinical remission, bone damage can still occur or progress, and bone damage is considered largely irreversible. To reduce the development of this bone damage, current treatment recommendations for RA support early and aggressive treatment. Would it therefore not be great if we could diagnose patients, of whom the bone is still deteriorating, at an earlier stage in order to initiate or change the treatment which may prevent progression of bone damage, and thus prevent loss of function and mobility? Besides the benefits on the individual level, improved diagnosis and monitoring of patients with RA could also benefit the society by reducing the costs that are related to RA, such as loss of productivity.

Imaging of peri-articular bone in RA in clinical practice is performed using conventional radiography $(C R)$, but $C R$ is not sensitive to detect bone damage at an early stage. More sensitive techniques, such as magnetic resonance imaging (MRI) or clinical CT, are suggested for monitoring disease activity and progression of bone damage. HR-pQCT is a novel three-dimensional imaging technique that allows in vivo evaluation of the bone at the micro-scale. HR-pQCT is proven to be a more sensitive technique in the detection of bone damage compared to $\mathrm{CR}, \mathrm{MRI}$ and clinical $\mathrm{CT}$. In this thesis, we further validated and improved the applicability of HR-pQCT imaging by introducing a more standardized and less time-consuming analysis of bone damage of finger joints. We showed that HRpQCT imaging with our introduced algorithm is a valid, reliable and applicable tool in the detection of small cortical interruptions, bone density and micro-structure in finger joints, and can discriminate between patients with RA and healthy subjects both crosssectionally and longitudinally.

\section{Contributions of this thesis to society}

The algorithm introduced in this thesis can be a significant contributor for further research, since it improves the reliability, applicability and discriminatory value of HRpQCT imaging in research of peri-articular bone in RA. 
We first showed that the HR-pQCT with our algorithm is able to detect smaller interruptions compared to CR, MRI and visual scoring of HR-pQCT images. Detection of small cortical interruptions is of importance since these small interruptions are more frequently present in patients with RA compared to healthy subjects. Therefore, HRpQCT imaging with our algorithm was of added value over findings on CR and MRI in the discrimination between patients with RA and healthy subjects. Additionally, these small interruptions may play a role in the development of large (pathological) interruptions (i.e. erosions). When large interruptions indeed develop at the 'entry points' through small physiological interruptions (such as vascular channels), HR-pQCT imaging will enable to show and follow these bone changes and occurrence of new cortical interruptions over time. This monitoring may be of additional value after treatment initiation in clinical studies in order to assess the effect of treatment, even early in this process. Because HR-pQCT has a higher sensitivity to detect bone damage (small cortical interruptions) and structural bone changes over time compared to $C R$ and $M R I$, it is expected that fewer participants will be needed in clinical trials with HR-pQCT to observe an effect of drug treatment. We already could identify differences in bone changes between patients treated with biological disease modifying anti-rheumatic drugs (bDMARDs) and synthetic DMARDs (sDMARDs).

Second, we showed a higher reliability of the algorithm compared to visual scoring. An improvement in reliability enables to better discriminate between patients and healthy subjects and to measure change over time. Therefore, compared to $C R, M R I$ and visual scoring of HR-pQCT images, we expect that fewer participants will be needed in clinical trials using HR-pQCT with our algorithm to observe an effect of drug treatment.

Third, the use of our algorithm is more applicable compared to visual scoring. With our algorithm, corrections of the outer cortical contours are needed to assess the number and size of interruptions. However, these corrections were already necessary to determine the bone density and micro-structural parameters. Hence, compared to visual scoring no extra visual evaluation to score interruptions is necessary. Improvement in applicability can help larger studies using HR-pQCT to analyse the data, and improvement in applicability is needed for eventual use in clinical practice.

This thesis aimed to answer the filters of the OMERACT initiative (outcome measures of rheumatology), defined by "truth", "discrimination" and "feasibility". The SPECTRA (Study grouP for xtrEmeCT in RA) collaboration, in which we participated, strives to implement HR-pQCT as imaging tool in clinical practice. A new instrument such as HRPQCT needs to meet these OMERACT filters, before it can be implemented in clinical practice. Other international scientific centres that are part of the SPECTRA initiative are interested to apply our cortical interruption detection algorithm. The results in this thesis form a solid base for further development and implementation of automated algorithms in the assessment of peri-articular bone damage in finger joints. 


\section{Future perspectives}

Currently, HR-pQCT imaging has only been used in research settings. Further optimisation of the algorithm in reliability and applicability, and confirmation of our results are needed before studying the possibility of use in clinical settings. Studies that further answer the questions raised by the OMERACT filters, "truth", "discrimination" and "feasibility", to show that HR-pQCT imaging is valid, reliable, applicable tool which can discriminate between situations of interest.

HR-pQCT imaging may be applied in several future studies, for example for in randomized controlled clinical trials that compare different treatment strategies. Other studies compared sDMARDs to bDMARDs, or the additional effect of osteoporosis medication treatment (e.g. denosumab or alendronate) in addition to DMARD treatment. Partial repair of bone was regularly observed using HR-pQCT in patients primarily on bDMARD treatment. Differences could already be observed after 3 months of treatment. In view of the limited availability of HR-pQCT, such studies could be performed in the context of pilot studies, proof of concept studies or Phase IV monitoring studies.

Further HR-pQCT research may focus on better identification of patients that will develop severe, erosive RA, and a variety of factors can be investigated using HR-pQCT to identify those patients (e.g. the number, size, location, shape and distribution of cortical interruptions, which may be predictors for the development of erosions). The structural integrity of the bone tissue around a cortical interruption might be an additional predictor of bone damage progression that can be studied with HR-pQCT.

HR-pQCT imaging may also be used in the future as a diagnostic tool to identify those patients that will develop RA and might be used to monitor patients with RA to earlier detect bone damage progression. This might be an indication to alter the medication of these patients to prevent bone damage progression.

This thesis provides a solid basis for an algorithm based analysis of HR-pQCT images of peri-articular bone in finger joints of patients with RA and healthy subjects, which can be further explored in future research, including other diseases that affect finger joints, such as psoriatic arthritis and erosive osteoarthritis.

\section{Current publicity}

Results in this thesis have been submitted and published in scientific journals. Results have also been presented at national and international conferences. Oral presentations have been given at the national conferences of the Dutch and Belgian Rheumatology association (Nederlandse Vereniging voor Reumatologie (NVR) and Belgian Congress of Rheumatology (BCR) (NVR in 2014 and 2017, and BCR in 2014 and 2015). Posters have been presented at American Society of Bone Mineral Research (ASBMR 2014), the European League Against Rheumatism (EULAR 2015, 2016 and 2017) and American College of Rheumatology (2016). 


\section{SUMMARY}

\section{Background}

Rheumatoid arthritis (RA) is a chronic inflammatory auto-immune disease, in which inflammation at the joint might lead to peri-articular osteoporosis and erosions (i.e. pathological cortical interruptions). ${ }^{1,2}$ Approximately $1 \%$ of the population worldwide suffers from rheumatoid arthritis (RA), and the disease is more common in females., Traditionally imaging in RA is performed using conventional radiography (CR), which is fast, inexpensive, easily accessible, provides a two dimensional (2D) assessment of bone, and rheumatologists are familiar with its interpretation. ${ }^{5}$ A major drawback of CR is that it is not sensitive to detect erosions at an early stage and (subtle) progression over time. ${ }^{6-9}$ More sensitive techniques to detect early structural changes in the joints are computed tomography (CT), ultrasound (US) and magnetic resonance imaging (MRI). ${ }^{10-13}$ In contrast to $C R$, US and MRI can visualize soft tissue, which enables detection of inflammation in the synovial fluid (synovitis). ${ }^{12,14-17} \mathrm{MRI}$ also enables to evaluate the presence of inflammation in the bone marrow (bone marrow edema (BME)). ${ }^{12,15-17}$. The (sole) use of US and MRI also has disadvantages, such as operator dependency (US), inability to penetrate bone tissue for imaging (US), limited availability (MRI), long duration of the examination (MRI) and the costs (MRI). ${ }^{10}$ The optimal imaging method in RA would be more sensitive and reproducible for diagnosis and monitoring changes in bone structure even at the smallest level. ${ }^{10}$

High-resolution peripheral quantitative CT (HR-pQCT) is a novel three-dimensional (3D) imaging technique with a resolution of $82 \mu \mathrm{m}$ that allows in vivo evaluation of the volumetric bone mineral density (VBMD) and bone micro-structure of the peripheral skeleton. ${ }^{18}$ It has been reported that multiple erosions can be seen in finger joints of patients with RA on HR-pQCT while having no erosions visible on CR, US and MRI, ${ }^{19-23}$ indicating that HR-pQCT is more sensitive in the detection of structural bone damage in RA than these imaging techniques. In addition to detection of erosions, HR-pQCT imaging is able to detect small cortical interruptions $(<0.5 \mathrm{~mm}$ in diameter $),{ }^{19}$ which may be the first signs of pathological interruptions (i.e. erosions). ${ }^{1,24,25}$ For these reasons, HR-pQCT imaging is a promising tool for diagnosis and/or monitoring of peri-articular bone in finger joints of patients with RA.

To date, cortical interruptions on HR-pQCT are detected by visual evaluation on multiple HR-pQCT slices, which is not only prone to subjectivity but also time-consuming. ${ }^{19,22,26-32}$ An automated scoring algorithm, for the detection of cortical interruptions, could potentially improve reliability by minimizing reader interventions and feasibility in clinical practice. In addition, VBMD and bone micro-structural parameters have not yet been studied in detail in finger joints of patients with RA compared to healthy subjects.

When developing a new tool, important aspects described in the Outcome Measures in Rheumatology (OMERACT) filter need to be taken into account: any instrument needs to meet the requirements defined by truth (validity), discrimination (reliability and 
discrimination between patients with RA and healthy subjects), and feasibility (applicability). ${ }^{33}$ In this thesis we therefore studied aspects of validity, reliability, discrimination and applicability of HR-pQCT in the detection of small cortical interruptions, VBMD and bone micro-structure in finger joints of patients with RA and healthy subjects.

\section{Summary of thesis}

Previous studies using HR-pQCT in RA mainly focused on erosions. ${ }^{19,22,23,26-31}$ Occasionally the existence of small, possible physiological vascular channels was reported, also in healthy individuals. ${ }^{19,22}$ Hence, there was a knowledge gap regarding the performance of HR-pQCT in the visual detection of small cortical interruptions. In Chapter 2, we therefore investigated the validity and reliability of visual detection of cortical interruptions in metacarpophalangeal (MCP) and proximal interphalangeal (PIP) joints when using HR-pQCT with microCT $(\mu \mathrm{CT})$ as gold standard. Nineteen joints of female anatomic specimens (ten MCP and nine PIP joints) were analyzed for the presence of cortical interruptions on HR-pQCT and $\mu \mathrm{CT}$. We studied the intra- and inter-reader reliability and determined the sensitivity, specificity, positive predictive value (PPV) and negative predictive value (NPV). Using either imaging modality, cortical interruptions were found in all joints (129 on HR-pQCT and 149 on $\mu \mathrm{CT}$ ). Intra-reader reliability for the presence (kappa, k) and total number (intraclass correlation coefficient, ICC) of cortical interruptions was moderate to substantial (range 0.52-0.61) for HR-pQCT. Inter-reader reliability for the presence $(\mathrm{K})$ and total number (ICC) of cortical interruptions was fair to moderate (range 0.37-0.55) for HR-pQCT. HR-pQCT appeared highly sensitive (81.6\%) to detect cortical interruptions with $\mu \mathrm{CT}$ as gold standard and the PPV was $81.6 \%$. In conclusion, cortical interruptions were commonly detected in finger joints on both HRpQCT and $\mu \mathrm{CT}$ with fair to substantial reliability and high sensitivity.

These findings in combination with the time consuming aspect of visual evaluation of HR-pQCT images ${ }^{34}$ indicated the need for a more reliable and feasible scoring method. While there was one published paper reporting accurate detection of the volume of erosions in finger joints on HR-pQCT images with an automated algorithm that relied on operator input, ${ }^{35}$ a fully-automated algorithm to detect cortical interruptions was not available yet. In Chapter 3, we therefore developed a fully-automated algorithm to detect cortical interruptions with a diameter $\geq 0.246 \mathrm{~mm}$ on HR-pQCT and tested it in MCP joints of patients with RA and healthy subjects from the MOSA-Hand cohort. ${ }^{36}$ Also, the additional value of manual correction of automatically generated outer cortical contours was investigated (semi-automated approach). The median number of cortical interruptions per joint detected with the algorithms was 14 (range 2-59) and did not differ significantly between the semi- and fully-automated algorithms ( $p=0.37)$. Almost perfect reliability (ICC 0.95-0.97) to detect cortical interruptions was observed for both the fully- and semi-automated algorithm procedures. Compared to visual detection of cortical interruptions, considered as the gold standard in this study, agreement of the 
fully- and semi-automated algorithms was fair to moderate (ICC 0.38-0.45). The majority of the interruptions detected visually were also detected by the fully- and semiautomated algorithms (70-76\%). Based on the findings in this study, the semi-automated procedure is the method of choice because manual corrections of the cortical contours are necessary in case of large cortical interruptions and considerable motion artefacts. We concluded that the application of the semi-automated algorithm is promising for a sensitive and objective assessment of cortical interruptions in fingers joints by HR-pQCT. In Chapter 4, we further validated our semi-automated algorithm by comparing the detection of cortical interruptions with different minimum cut-off diameters (range $\geq 0.246 \mathrm{~mm}$ to $\geq 0.574 \mathrm{~mm}$ ) on HR-pQCT to $\mu \mathrm{CT}$, as gold standard, using ten female index fingers from anatomic specimens. In Chapter 4, cut-off values for defining a cortical interruption were $>0.164 \mathrm{~mm}$ (i.e. $>2$ voxels $\equiv \geq 3$ voxels $\equiv \geq 0.246 \mathrm{~mm}$ ), $>0.328 \mathrm{~mm}$ (i.e. $>4$ voxels $\equiv \geq 5$ voxels $\equiv \geq 0.328 \mathrm{~mm}$ ) and $>0.492 \mathrm{~mm}$ (i.e. $>6$ voxels $\equiv \geq 7$ voxels $\equiv$ $\geq 0.574 \mathrm{~mm}$ ). In addition, validity of single interruptions with different minimum diameters on HR-pQCT was evaluated through calculating PPV and sensitivity with a fixed interruption diameter of $\geq 0.100 \mathrm{~mm}$ on $\mu C T\left(P P V_{0.10 \mathrm{~mm}}\right.$ and sensitivity $\left.y_{0.10 \mathrm{~mm}}\right)$ and same minimum cortical interruption diameter $\left(\mathrm{PPV}_{\mathrm{sd}}\right.$ and sensitivity $\left.\mathrm{s}_{\mathrm{sd}}\right)$.

On the joint level, almost perfect agreement for the number and surface area of interruptions was obtained for interruptions with a minimum diameter of $\geq 0.246 \mathrm{~mm}$ and $\geq 0.410 \mathrm{~mm}$ (ICC $\geq 0.81$ ). A fair to moderate agreement was found for interruptions $\geq 0.574 \mathrm{~mm}$ (ICC $\leq 0.52$ ). At the level of a single interruption, we found that the $P P V_{0.10 \mathrm{~mm}}$ improved with every increase in minimum cortical interruption diameter from 84.9 to $100 \%$, for interruptions $\geq 0.246 \mathrm{~mm}$ to $\geq 0.574 \mathrm{~mm}$, respectively. However, sensitivity $0_{0.10 \mathrm{~mm}}$ of these small interruptions decreased (from 62.4 to $4.7 \%$ ) with every increase in minimum cortical interruption diameter. PPV $\mathrm{sd}_{\mathrm{sd}}$ was substantial for interruptions with a diameter of $\geq 0.246 \mathrm{~mm}$ and $\geq 0.41 \mathrm{~mm}\left(\mathrm{PPV}_{\mathrm{sd}}=66.6\right.$ and $66.3 \%$, respectively), but fair for interruptions with a diameter of $\geq 0.574 \mathrm{~mm}$ ( $\mathrm{PPV}_{\mathrm{sd}}=38.5 \%$ ). Sensitivity $_{\text {sd }}$ was substantial for interruptions with a diameter of $\geq 0.246 \mathrm{~mm}$ and $\geq 0.574 \mathrm{~mm}$ (Sensitivity ${ }_{s d}=79.1$ and $73.5 \%$, respectively), but moderate for interruptions with a diameter of $\geq 0.41 \mathrm{~mm}$ (Sensitivity ${ }_{\text {sd }}=49.4 \%$ ).

Hence, the size of an interruption detected on HR-pQCT may be different from that on $\mu \mathrm{CT}$. The semi-automated algorithm for detection of cortical interruptions with HR-pQCT in finger joints performed best for interruptions with a minimum diameter of $\geq 0.246$ and $\geq 0.41 \mathrm{~mm}$. Almost perfect agreement was obtained at the joint level and the interruptions matched well with those detected on $\mu \mathrm{CT}$ for both cut-off values. We used a diameter cut-off of $\geq 0.41 \mathrm{~mm}$ in the subsequent studies, since we preferred a high $\mathrm{PPV}_{0.10 \mathrm{~mm}}$ when extending the algorithm with trabecular void volume detection.

So far, our algorithm only analyzed the presence of an interruption in the cortex and did not consider the underlying trabecular bone void as part of the total interruption volume. In addition, the reproducibility of our algorithm and the cortical micro-structural parameters on repeated scans with complete repositioning between the scans has not yet been tested in the standard workflow of the HR-pQCT scanner. Therefore, in 
Chapter 5, we extended our algorithm for detection of cortical interruptions with underlying trabecular bone void volume detection, and evaluated the reproducibility of the extended algorithm, the $\mathrm{VBMD}$, and micro-structural parameters of the cortical and trabecular region. For this study, we used HR-pQCT images from the Early Inflammatory Arthritis Clinics from the Division of Rheumatology at the University of Calgary, who recruited twenty-one patients (mean age 49 (standard deviation: 11) years) with early RA $(n=17)$ and undifferentiated arthritis $(n=4)$, all diagnosed less than one year ago.

The reproducibility of the extended algorithm for the number, surface area and volume of cortical interruptions was excellent (ICCS $\geq 0.82$ ), especially for the interruption volume (ICC 0.99). The absolute least significant change ( LSC $_{S D}$ ) values were 4.2 for the number of interruptions, $5.8 \mathrm{~mm}^{2}$ for interruption surface, and $3.2 \mathrm{~mm}^{3}$ for the interruption volume. The reproducibility for the VBMD and bone micro-structural parameters was excellent with ICCS $\geq 0.97$, and good for the cortical porosity diameter (Ct.Po.Dm, ICC $=0.84)$. The percentage $L S C$ values $\left(L_{S C} C_{C V \%}\right)$ were $\leq 7.3 \%$ for the $\mathrm{VBMD}$ parameters and $\leq 12.1 \%$ for the micro-structural parameters, except for Tb.SpSD, Ct.Po.Dm and Ct.Po which were between $21.1 \%$ and $24.1 \%$. Therefore, we concluded that the algorithm was highly reproducible but still had substantial precision errors while the assessment of the VBMD and bone micro-structural parameters was highly reproducible with acceptable precision errors.

Whereas these earlier studies demonstrated the validity, reliability and applicability of our semi-automated algorithm in quantifying (small) cortical interruptions, it remained unknown whether they were related to both structural damage on $C R$, and structural damage and inflammatory markers on MRI. In addition, it was unknown if HR-pQCT imaging had an added value over these findings on CR and MRI in the detection of (small) cortical interruptions and in the distinction between patients with RA and healthy subjects. Therefore, in Chapter 6, we compared the number and size of cortical interruptions in patients with RA to healthy subjects using the semi-automated algorithm. Additionally, we investigated the association between structural damage and inflammatory markers on either CR or MRI with cortical interruptions on HR-pQCT. Also, the added value of HR-pQCT over these findings on CR and MRI was investigated. For this cross-sectional study, we used data from the MOSA-Hand cohort, which consists of 38 healthy females and 39 female patients diagnosed with RA (mean age (SD) 51.2 (10.3) and 54.8 (7.0), respectively). ${ }^{36}$

Significantly more cortical interruptions and both a significantly larger surface area and volume of cortical interruptions were detected in MCP joints of patients with RA compared to healthy subjects. Findings on CR and MRI were significantly associated with the number, surface area and volume of cortical interruptions on HR-pQCT (prevalence ratios (PRs) ranging from 1.03 to 7.48 , all $p<0.01$ ). Having RA was significantly associated with the number, surface area and volume of cortical interruptions on HR-pQCT (PRs 2.26 to 5.39 , all $p<0.01$ ), also after adjustment for findings on CR or MRI. We therefore concluded that HR-pQCT imaging with the semi-automated algorithm may be of value in addition to $C R$ and MRI for the evaluation of structural damage in patients with RA. 
Cross-sectional discrimination between patients with RA and healthy subjects was found using HR-pQCT, but the longitudinal discriminatory value of HR-pQCT remained unknown. Therefore, in Chapter 7, we prospectively investigated the change in number, surface area and volume of cortical interruptions, VBMD- and bone micro-structural parameters in patients with RA (treated with synthetic disease modifying anti-rheumatic drugs (sDMARDs) and/or biologic DMARDs (bDMARDs)) after a 1-year follow-up period, and compared our results with a population of healthy subjects. Eleven of the 77 patients declined participation at the second visit and two subjects were excluded because no HR-pQCT images were of sufficient quality. In total, 32 healthy subjects and 32 patients with RA could be evaluated.

The number, surface area and volume of interruptions did not change at the group level in both patients with RA and healthy subjects, except for a significant increase in the number of interruptions in PIP joints of patients with RA. The decrease in VBMD was significantly more in MCP and PIP joints of patients with RA $\left(-4.4\right.$ and $-5.8 \mathrm{mgHA} / \mathrm{cm}^{3}$, respectively) compared to healthy subjects (-1.1 and $-2.6 \mathrm{mgHA} / \mathrm{cm}^{3}$, respectively), and the trabecular thickness also decreased significantly more in PIP joints of patients with RA compared to healthy subjects.

Applying the LSC $_{S D}$ cut-off values, the proportion of joints showing repair was higher in patients with RA compared with healthy subjects $(4.1 \%, 3.6 \%$ and $6.6 \%$ versus $0 \%, 0 \%$ and $1.7 \%$, respectively), and was significant for the volume of interruptions (Odd's Ratio (OR) 6.6 (95\% Cl 1.08-39.9), $p=0.041$ ). The proportions of joints showing bone loss was not significantly different between the groups $(4.1 \%, 2.7 \%$ and $5.9 \%$ versus $0.9 \%, 0 \%$ and $2.6 \%$, respectively).

The proportion of joints showing progression in cortical interruption was higher in patients on sDMARDs compared with patients on bDMARDs (6.1\%, 3.0\% and $4.0 \%$ versus $1.8 \%, 1.8 \%$ and $7.9 \%$ ), and was significant for the number of interruptions (OR: 6.6 (95\% $\mathrm{Cl} 1.0-41.7), p=0.048)$. The proportion of joints with loss in $\mathrm{VBMD}$ beyond $\mathrm{LSC}_{S D}$ was significantly higher in patients treated with sDMARDs compared with bDMARDs $(31.3 \%$ versus 17.2\%, OR 2.7 (95\% Cl 1.4-5.3), $p=0.004$, respectively).

We concluded that, HR-pQCT is able to quantify bone repair and loss in finger joints in RA and HS. Cortical interruption-, vBMD- and micro-structural parameters were impaired in patients with RA compared with $\mathrm{HS}$, of which $\mathrm{VBMD}$ and micro-structural parameters further deteriorated. Furthermore, in patients treated with sDMARDs significantly more decrease in VBMD compared with patients treated with bDMARDs was found. HR-pQCT with our semi-automated algorithm showed promising results for monitoring treatment effects in RA.

Finally, in Chapter 9, the main findings were summarized and discussed in the context of the OMERACT determined terms "validity", "reliability", "discriminatory value" and "applicability" for detection of cortical interruptions with the semi-automated algorithm and the assessment of VBMD and bone micro-structural parameters.

Exploring the "validity", HR-pQCT was sensitive in the detection of cortical interruptions compared to $\mu \mathrm{CT}$ (Chapter 2). In Chapter 3, significantly more cortical interruptions were 
detected with the algorithm compared to visual scoring. Differences between visual scoring and the semi-automated algorithm were mainly found in regions of low cortical bone density, where it is often difficult to detect interruptions visually, while the algorithm can detect these interruptions more easily on binary images. On the other hand, due to partial volume effects (PVEs) interruption size may be overestimated. In Chapter 4, false positive detection of cortical interruptions could be avoided by increasing the minimal cut-off diameter of interruptions. In Chapter 6, validity was further investigated by comparing the semi-automated algorithm to clinical standards ( $C R$ and MRI). Structural damage on both $C R$ and MRI were associated with the number and size of cortical interruptions on HR-pQCT.

Exploring the "reliability", we found that the inter-reader reliability was fair to moderate in the visual scoring of cortical interruptions. In Chapter 3, the reliability of the algorithm (semi- and fully-automated) in the detection of cortical interruptions was higher compared with visual scoring (Chapter 2). However, the fully-automated algorithm was susceptible to errors due to motion artefacts and in case of large cortical interruptions. In Chapter 5, we found that the reproducibility of the semi-automated algorithm was excellent on repeated scans and precision errors were substantial compared to the mean values detected. In general we found a higher reliability with the semi-automated algorithm compared with visual scoring in the number and size of cortical interruptions. Precision errors were comparable to those reported by others, although in contrast to previous studies we also analyzed small cortical interruptions. ${ }^{33}$ Additionally, we found an excellent inter-operator reliability and intra-operator reproducibility, comparable to earlier studies. In summary, we found that it is reliable to use our semi-automated algorithm for detection of cortical interruptions, and assessment of VBMD and bone micro-structural parameters on HR-pQCT images of finger joints.

Exploring the "discriminatory value", we found more and larger cortical interruptions already in early RA patients (disease duration <2 years) compared to healthy subjects (Chapter 6). The presence of RA was associated with more and larger interruptions even after adjustment of structural damage on $\mathrm{CR}$ and $\mathrm{MRI}$ and inflammatory markers on MRI. The longitudinal change in patients with RA compared with healthy subjects after 1 year follow-up showed no change at the group level in both groups. However, at the level of individual joints, we found bone repair or bone damage progression, both mainly in patients with RA. Furthermore, we looked at the discriminatory value of the VBMD and bone micro-structural parameters. We found an impaired VBMD and bone microstructure in patients with RA compared to healthy subjects. The VBMD and bone microstructure deteriorated more in patients with RA compared with healthy subjects after 1 year follow-up. The decrease of $V B M D$ was greater in RA patients treated with sDMARDs compared to bDMARDs.

Finally, the "applicability" of HR-pQCT imaging was determined. Previously we have reported that visual scoring was not feasible in a clinical setting because it was very time consuming. ${ }^{34}$ The use of our semi-automated algorithm may reduce examination time. Correction of the outer cortical contour remains necessary for instance in case of motion 
artefacts and the large cortical interruptions. This contour correction is also a standard procedure for HR-pQCT measurements of the distal radius and tibia. However, after correction of the contours, the number and size of cortical interruptions, VBMD and bone micro-structural parameters can be assessed automatically.

In conclusion, this thesis showed that semi-automated analysis of HR-pQCT images is a valid, reliable and applicable tool which can discriminate between patients with RA and healthy subjects in the detection of cortical interruptions (diameter $\geq 0.41 \mathrm{~mm}$ ), and in the assessment of $\mathrm{VBMD}$ and bone micro-structural parameters in finger joints. Therefore, HR-pQCT imaging with our algorithm may contribute in the evaluation of peri-articular bone in finger joints of patients with RA. 


\section{REFERENCES}

1. Schett G, Gravallese E. Bone erosion in rheumatoid arthritis: mechanisms, diagnosis and treatment. Nat Rev Rheumatol. 2012;8(11):656-64.

2. Geusens P, van den Bergh J. Bone erosions in rheumatoid arthritis. Rheumatology. 2014;53(1):4-5.

3. Myasoedova E, Crowson CS, Kremers HM, Therneau TM, Gabriel SE. Is the incidence of rheumatoid arthritis rising?: results from Olmsted County, Minnesota, 1955-2007. Arthritis Rheum. 2010;62(6): 1576-82.

4. Alamanos Y, Voulgari PV, Drosos AA. Incidence and prevalence of rheumatoid arthritis, based on the 1987 American College of Rheumatology criteria: a systematic review. Semin Arthritis Rheum. 2006;36(3):182-8.

5. van der Heijde DM. Radiographic imaging: the 'gold standard' for assessment of disease progression in rheumatoid arthritis. Rheumatology. 2000;39 Suppl 1:9-16.

6. Mottonen TT. Prediction of erosiveness and rate of development of new erosions in early rheumatoid arthritis. Ann Rheum Dis. 1988;47(8):648-53.

7. van der Heijde DM, van Leeuwen MA, van Riel PL, Koster AM, van 't Hof MA, van Rijswijk MH, et al. Biannual radiographic assessments of hands and feet in a three-year prospective followup of patients with early rheumatoid arthritis. Arthritis Rheum. 1992;35(1):26-34.

8. Nissila M, Isomaki H, Kaarela K, Kiviniemi P, Martio J, Sarna S. Prognosis of inflammatory joint diseases. A three-year follow-up study. Scand J Rheumatol. 1983;12(1):33-8.

9. Ravindran V, Rachapalli S. An overview of commonly used radiographic scoring methods in rheumatoid arthritis clinical trials. Clin Rheumatol. 2011;30(1):1-6.

10. Ostergaard M, Pedersen SJ, Dohn UM. Imaging in rheumatoid arthritis--status and recent advances for magnetic resonance imaging, ultrasonography, computed tomography and conventional radiography. Best practice \& research Clin Rheumatol. 2008;22(6):1019-44.

11. Baillet A, Gaujoux-Viala C, Mouterde G, Pham T, Tebib J, Saraux A, et al. Comparison of the efficacy of sonography, magnetic resonance imaging and conventional radiography for the detection of bone erosions in rheumatoid arthritis patients: a systematic review and meta-analysis. Rheumatology. 2011; 50(6):1137-47.

12. Dohn UM, Ejbjerg BJ, Court-Payen M, Hasselquist M, Narvestad E, Szkudlarek M, et al. Are bone erosions detected by magnetic resonance imaging and ultrasonography true erosions? A comparison with computed tomography in rheumatoid arthritis metacarpophalangeal joints. Arthritis Res Ther. 2006;8(4):R110.

13. Perry D, Stewart N, Benton N, Robinson E, Yeoman S, Crabbe J, et al. Detection of erosions in the rheumatoid hand; a comparative study of multidetector computerized tomography versus magnetic resonance scanning. J Rheumatol. 2005;32(2):256-67.

14. Szkudlarek M, Klarlund M, Narvestad E, Court-Payen M, Strandberg C, Jensen KE, et al. Ultrasonography of the metacarpophalangeal and proximal interphalangeal joints in rheumatoid arthritis: a comparison with magnetic resonance imaging, conventional radiography and clinical examination. Arthritis Res Ther. 2006;8(2):R52.

15. Conaghan PG, O'Connor P, McGonagle D, Astin P, Wakefield RJ, Gibbon WW, et al. Elucidation of the relationship between synovitis and bone damage: a randomized magnetic resonance imaging study of individual joints in patients with early rheumatoid arthritis. Arthritis Rheum. 2003;48(1):64-71.

16. Ostergaard M, Stoltenberg M, Lovgreen-Nielsen P, Volck B, Jensen CH, Lorenzen I. Magnetic resonance imaging-determined synovial membrane and joint effusion volumes in rheumatoid arthritis and osteoarthritis: comparison with the macroscopic and microscopic appearance of the synovium. Arthritis Rheum. 1997;40(10):1856-67.

17. Ostendorf B, Peters R, Dann P, Becker A, Scherer A, Wedekind F, et al. Magnetic resonance imaging and miniarthroscopy of metacarpophalangeal joints: sensitive detection of morphologic changes in rheumatoid arthritis. Arthritis Rheum. 2001;44(11):2492-502.

18. Boutroy S, Bouxsein ML, Munoz F, Delmas PD. In vivo assessment of trabecular bone microarchitecture by high-resolution peripheral quantitative computed tomography. J Clin Endocrinol Metab. 2005;90(12):6508-15. 
19. Stach CM, Bauerle M, Englbrecht M, Kronke G, Engelke K, Manger B, et al. Periarticular bone structure in rheumatoid arthritis patients and healthy individuals assessed by high-resolution computed tomography. Arthritis Rheum. 2010;62(2):330-9.

20. Fouque-Aubert A, Boutroy S, Marotte H, Vilayphiou N, Bacchetta J, Miossec P, et al. Assessment of hand bone loss in rheumatoid arthritis by high-resolution peripheral quantitative CT. Ann Rheum Dis. 2010;69(9):1671-6.

21. Lee CH, Srikhum W, Burghardt AJ, Virayavanich W, Imboden JB, Link TM, et al. Correlation of structural abnormalities of the wrist and metacarpophalangeal joints evaluated by high-resolution peripheral quantitative computed tomography, 3 Tesla magnetic resonance imaging and conventional radiographs in rheumatoid arthritis. Int J Rheum Dis. 2015;18(6):628-39.

22. Finzel S, Ohrndorf S, Englbrecht M, Stach C, Messerschmidt J, Schett G, et al. A detailed comparative study of high-resolution ultrasound and micro-computed tomography for detection of arthritic bone erosions. Arthritis Rheum. 2011;63(5):1231-6.

23. Albrecht A, Finzel S, Englbrecht M, Rech J, Hueber A, Schlechtweg $P$, et al. The structural basis of MRI bone erosions: an assessment by microCT. Ann Rheum Dis. 2013;72(8):1351-7.

24. Binks DA, Gravallese EM, Bergin D, Hodgson RJ, Tan AL, Matzelle MM, et al. Role of vascular channels as a novel mechanism for subchondral bone damage at cruciate ligament entheses in osteoarthritis and inflammatory arthritis. Ann Rheum Dis. 2015;74(1):196-203.

25. Schett G, Stolina M, Bolon B, Middleton S, Adlam M, Brown H, et al. Analysis of the kinetics of osteoclastogenesis in arthritic rats. Arthritis Rheum. 2005;52(10):3192-201.

26. Regensburger A, Rech J, Englbrecht M, Finzel S, Kraus S, Hecht K, et al. A comparative analysis of magnetic resonance imaging and high-resolution peripheral quantitative computed tomography of the hand for the detection of erosion repair in rheumatoid arthritis. Rheumatology (Oxford). 2015; 54(9):1573-81.

27. Lee CH, Srikhum W, Burghardt AJ, Virayavanich W, Imboden JB, Link TM, et al. Correlation of structural abnormalities of the wrist and metacarpophalangeal joints evaluated by high-resolution peripheral quantitative computed tomography, 3 Tesla magnetic resonance imaging and conventional radiographs in rheumatoid arthritis. Int J Rheum Dis. 2015;18(6):628-39.

28. Finzel S, Rech J, Schmidt S, Engelke K, Englbrecht M, Stach C, et al. Repair of bone erosions in rheumatoid arthritis treated with tumour necrosis factor inhibitors is based on bone apposition at the base of the erosion. Ann Rheum Dis. 2011;70(9):1587-93.

29. Finzel S, Rech J, Schmidt S, Engelke K, Englbrecht M, Schett G. Interleukin-6 receptor blockade induces limited repair of bone erosions in rheumatoid arthritis: a micro CT study. Ann Rheum Dis. 2013;72(3):396-400.

30. Yue J, Griffith JF, Xiao F, Shi L, Wang D, Shen J, et al. Repair of bone erosion in rheumatoid arthritis by denosumab: A high-resolution peripheral quantitative computed tomography study. Arthritis Care Res. 2017;69(8):1156-63.

31. Srikhum W, Virayavanich W, Burghardt AJ, Yu A, Link TM, Imboden JB, et al. Quantitative and semiquantitative bone erosion assessment on high-resolution peripheral quantitative computed tomography in rheumatoid arthritis. J Rheumatol. 2013;40(4):408-16.

32. Zebaze R, Seeman E. Cortical bone: a challenging geography. J Bone Miner Res. 2015;30(1):24-9.

33. Boers M, Brooks P, Strand CV, Tugwell P. The OMERACT filter for Outcome Measures in Rheumatology. J Rheumatol. 1998;25(2):198-9.

34. Scharmga A. High-resolution peripheral quantitative computed tomography in patients with rheumatoid arthritis: truth, discrimination and feasibility. Thesis UM 2017.

35. Topfer D, Finzel S, Museyko O, Schett G, Engelke K. Segmentation and quantification of bone erosions in high-resolution peripheral quantitative computed tomography datasets of the metacarpophalangeal joints of patients with rheumatoid arthritis. Rheumatology. 2014;53(1):65-71.

36. Scharmga A, Peters M, van Tubergen A, van den Bergh J, Barnabe C, Finzel S, et al. Heterogeneity of Cortical Breaks in Hand Joints of Patients with Rheumatoid Arthritis and Healthy Controls Imaged by High-resolution Peripheral Quantitative Computed Tomography. J Rheumatol. 2016;43(10):1914-20. 


\section{NEDERLANDSE SAMENVATTING}

\section{Achtergrond}

Reumatoïde artritis (RA) is een chronische ontstekingsziekte, waarbij ontsteking leidt tot afbraak van het bot wat kan resulteren in schade aan het bot (erosies, corticale interrupties) of botverlies (osteoporose). ${ }^{1,2}$ Ongeveer $1 \%$ van de wereldbevolking wordt getroffen door RA en de ziekte komt vaker voor bij vrouwen. ${ }^{3,4}$ In de kliniek wordt beeldvorming bij patiënten met RA vooral uitgevoerd met een röntgenfoto van de handen en voeten. Het vervaardigen van röntgenfoto's is eenvoudig, relatief goedkoop, de techniek is ruim beschikbaar en het geeft een twee dimensioneel beeld van het bot. ${ }^{5}$ Een nadeel van röntgenfoto's is echter dat het hiermee niet goed mogelijk is om vroege schade aan het bot en kleine veranderingen te detecteren. ${ }^{6-9}$ Gevoeligere methodes waarmee schade in het bot gedetecteerd kunnen worden zijn: computertomografie (CT), echografie en magnetische resonantie imaging (MRI). ${ }^{10-13}$ In tegenstelling tot de röntgenfoto kunnen echografie en MRI de weke delen in beeld brengen, waardoor ontsteking in het gewricht mede gedetecteerd kan worden. ${ }^{12,14-17}$ Daarnaast kan met MRI ook ontsteking van het beenmerg gedetecteerd worden (beenmerg oedeem). ${ }^{12,15-17}$ Het gebruik van echografie en MRI heeft echter ook nadelen, zoals operator afhankelijkheid (echografie), beperking om dieper in het bot te kijken (echografie), beperkte beschikbaarheid en de hoge kosten (MRI). De optimale methode voor beeldvorming van botaantasting in RA zou sensitiever en reproduceerbaarder moeten zijn in de diagnose en het monitoren van veranderingen in de botstructuur op micro niveau. $^{10}$

Hoge resolutie perifere kwantitatieve CT (HR-pQCT) is een nieuwe beeldvormende techniek met een resolutie van $82 \mu \mathrm{m}$ die het mogelijk maakt om driedimensionaal de botdichtheid en bot microstructuur te meten van het perifere skelet (distale radius en tibia). ${ }^{18}$ Eerdere studies rapporteerden dat met gebruik van HR-pQCT meerdere erosies werden gevonden in vinger gewrichten van patiënten met RA zonder dat er erosies te zien waren op de röntgenfoto's, echografie en MRI, ${ }^{19-23}$ wat erop wijst dat HR-pQCT sensitiever is in de detectie van structurele botschade in RA dan deze beeldvormende technieken. Met HR-pQCT kunnen naast erosies ook kleinere interrupties van het corticaal bot gedetecteerd worden $(<0.5 \mathrm{~mm}$ in diameter $) .{ }^{19}$ Deze kleine interrupties zijn mogelijk de eerste tekenen van erosies. ${ }^{1,24,25}$ Om deze redenen is HR-pQCT een veelbelovende techniek voor de diagnostiek en/of het monitoren van de botstructuur in de vingergewrichten van patiënten met RA.

Tot op heden zijn corticale interrupties met HR-pQCT gedetecteerd door middel van visuele evaluatie van de HR-pQCT beelden. Dit is niet alleen subjectief, maar ook tijdrovend. ${ }^{19,22,26-32}$ Een automatisch scoringsalgoritme dat corticale interrupties detecteert kan potentieel de betrouwbaarheid verbeteren en de toepasbaarheid in de kliniek vergroten door de interventies van een operator te beperken. Daarnaast zijn de 
botdichtheid en bot microstructuur parameters nog niet in detail bestudeerd in vingergewrichten van patiënten met RA in vergelijking tot gezonde proefpersonen.

Wanneer je een nieuwe methode ontwikkelt, moet je belangrijke aspecten die beschreven staan in de OMERACT ('Outcome Measures in Rheumatology') filter in acht nemen. leder instrument moet voldoen aan de voorwaarden gedefinieerd als 'waarheid' (validiteit), 'discriminatie' (betrouwbaarheid en discriminerend vermogen tussen patiënten en gezonde proefpersonen) en 'bruikbaarheid' (toepasbaarheid). ${ }^{33}$ In dit proefschrift hebben we daarom aspecten van de validiteit, betrouwbaarheid, het discriminerend vermogen en de toepasbaarheid van HR-pQCT bestudeerd in de detectie van kleine corticale interrupties alsmede de botdichtheid en bot microstructuur in vingergewrichten van patiënten met RA en gezonde proefpersonen.

\section{Samenvatting}

Eerdere onderzoeken met HR-pQCT in RA richtten zich vooral op erosies. ${ }^{19,22,23,26-31}$ Incidenteel werd het bestaan van kleine, mogelijk fysiologische vasculaire kanalen gerapporteerd, in patiënten met RA en ook in gezonde proefpersonen. ${ }^{19,22} \mathrm{Er}$ was dus een kennis kloof met betrekking tot de visuele detectie van kleine corticale interrupties. In hoofdstuk 2 hebben we daarom de validiteit en betrouwbaarheid van visuele detectie van corticale interrupties op HR-pQCT beelden van metacarpofalangeale (MCP) en proximale interfalangeale (PIP) gewrichten onderzocht met microCT $(\mu \mathrm{CT})$ als gouden standaard. Negentien gewrichten van anatomische vinger specimen (10 MCP en 9 PIP gewrichten) werden visueel geanalyseerd op de aanwezigheid van corticale interrupties op HR-pQCT en $\mu \mathrm{CT}$ door twee beoordelaars. De intra- en inter-beoordelaars betrouwbaarheid werden bestudeerd door middel van Cohen's kappa ( $\mathrm{k}$ ) en intra class correlatie coëfficiënt (ICC). Sensitiviteit, specificiteit, positief voorspellende waarde (PVW) en negatief voorspellende waarde (NVW) van HR-pQCT in de detectie van corticale interrupties werden berekend. Corticale interrupties werden in alle gewrichten gevonden met beide beeldmodaliteiten (129 op HR-pQCT en 149 op $\mu C T$ ). Intrabeoordelaar betrouwbaarheid voor de aanwezigheid ( $\mathrm{K}$ ) en het totale aantal (ICC) corticale interrupties was redelijk tot substantieel (bereik 0.52-0.61) voor HR-pQCT. Inter-beoordelaar betrouwbaarheid voor de aanwezigheid en het totale aantal corticale interrupties was matig tot redelijk (bereik 0.37-0.55) voor HR-pQCT. HR-pQCT bleek hoog sensitief te zijn (81.6\%) om corticale interrupties te detecteren met $\mu \mathrm{CT}$ als gouden standaard met een PVW van $81.6 \%$. We concludeerden dat corticale interrupties vaak werden gedetecteerd in vinger gewrichten met zowel HR-pQCT als $\mu \mathrm{CT}$ met een matige tot redelijke betrouwbaarheid en hoge sensitiviteit.

Deze bevindingen in combinatie met het tijdrovende aspect van visuele evaluatie van HR-pQCT beelden impliceerden dat een meer betrouwbare en toepasbare scoringsmethode nodig was. ${ }^{34}$ Hoewel in één artikel werd gerapporteerd over een automatisch algoritme die het volume van erosies in vingergewrichten op HR-pQCT beelden accuraat detecteert, was dit algoritme nog steeds afhankelijk is van de input van 
een operator $^{35}$ en was een volautomatisch algoritme dat corticale interrupties detecteert nog niet beschikbaar. In hoofdstuk 3, hebben we daarom een volautomatisch HR-pQCT algoritme ontwikkeld voor detectie van corticale interrupties met een diameter $\geq 0.246 \mathrm{~mm}$. We hebben het algoritme getest in HR-pQCT beelden van MCP gewrichten van patiënten met RA en gezonde proefpersonen van het MOSA-Hand cohort. De toegevoegde waarde van manuele correctie van de automatisch gegenereerde corticale contouren werd ook onderzocht (semiautomatische benadering). Het aantal corticale interrupties gedetecteerd per gewricht verschilde niet tussen de semi- en volautomatische benadering (mediaan 14 versus $15, p=0.37$ ) en een bijna perfecte betrouwbaarheid (ICC 0.95-0.97) werd gevonden. Visueel scoren werd in deze studie beschouwd als gouden standaard en de overeenstemming tussen de semi- en volautomatische algoritmes met visueel scoren was matig tot redelijk (ICC 0.38-0.45). De meerderheid van de interrupties die visueel werden gescoord werden ook gedetecteerd met de semi- en volautomatische algoritmes (sensitiviteit 70-76\%). Gebaseerd op de bevindingen in deze studie wordt de semiautomatische benadering aanbevolen omdat in het geval van grote corticale interrupties en bewegingsartefacten correcties van de automatisch gegenereerde buitenste contour nodig zijn. We concludeerden dat de toepassing van het semiautomatische algoritme veelbelovend is voor een sensitieve en objectieve bepaling van corticale interrupties in vingergewrichten met HR-pQCT beelden.

In hoofdstuk 4 hebben we het door ons ontwikkelde semiautomatische algoritme verder gevalideerd door de detectie van corticale interrupties met verschillende diameters als minimum afkapwaarde (bereik $\geq 0.246 \mathrm{~mm}$ tot $\geq 0.574 \mathrm{~mm}$ ) op HR-pQCT beelden te vergelijken met $\mu C T$ beelden als gouden standaard. Hierbij hebben we gebruik gemaakt van 10 wijsvingers (10 MCP en 9 PIP gewrichten) van anatomische specimens van vrouwen. Daarnaast hebben we de validiteit van de detectie van corticale interrupties met verschillende minimum afkapwaarden van diameters op HR-pQCT geëvalueerd door middel van het berekenen van de PVW en sensitiviteit ten opzicht van een gefixeerde diameter van interruptie van $\geq 0.10 \mathrm{~mm}$ op $\mu C T$ ( PVW $_{0.10 \mathrm{~mm}}$ en sensitiviteit $\mathrm{t}_{0.10 \mathrm{~mm}}$ ) en ten opzichte van dezelfde minimale diameter ( $P V W_{z d}$ en sensitiviteit $t_{z d}$ ).

Op gewrichtsniveau werd een bijna perfecte overeenstemming gevonden voor het aantal en de oppervlakte van interrupties met een minimale diameter van $\geq 0.246 \mathrm{~mm}$ en $\geq 0.410 \mathrm{~mm}$ (ICC $\geq 0.81$ ). Een matige tot redelijke overeenstemming werd gevonden voor interrupties $\geq 0.574 \mathrm{~mm}$ (ICC $\leq 0.52$ ). Voor afzonderlijke interrupties vonden we dat de $\mathrm{PVW}_{0.10 \mathrm{~mm}}$ verbeterde met iedere verhoging van de minimale afkapwaarde van diameter van corticale interrupties: van $84.9 \%$ tot $100 \%$ voor respectievelijk interrupties $\geq 0.246 \mathrm{~mm}$ tot $\geq 0.574 \mathrm{~mm}$. De sensitiviteit $t_{0.10 \mathrm{~mm}}$ van deze kleine interrupties nam echter af van $62.4 \%$ naar $4.7 \%$. De $\mathrm{PVW}_{\text {zd }}$ was substantieel voor interrupties met een diameter van $\geq 0.246 \mathrm{~mm}$ en $\geq 0.410 \mathrm{~mm}$ ( $P V W_{z d}$ respectievelijk $66.6 \%$ en $66.3 \%$ ), maar was matig voor interrupties met een minimale diameter van $\geq 0.574 \mathrm{~mm}\left(\mathrm{PPV}_{z d}=38.5 \%\right)$. De sensitiviteit $_{z d}$ was substantieel voor interrupties met een minimale diameter van 
$166 \mid$ Addendum

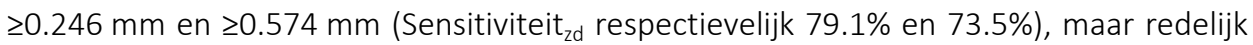
voor interrupties $\geq 0.410 \mathrm{~mm}$ (sensitiviteit $\mathrm{zd}_{\mathrm{d}}=49.4 \%$ ).

Dit betekent dat de grootte van een corticale interruptie die gedetecteerd wordt op HRpQCT verschillend kan zijn van die op $\mu C T$. Het semiautomatische HR-pQCT algoritme voor detectie van corticale interrupties in vingergewrichten presteerde het best voor interrupties met een minimale diameter van $\geq 0.246 \mathrm{~mm}$ en $\geq 0.410 \mathrm{~mm}$. Een bijna perfecte overeenstemming werd verkregen op het gewrichtsniveau en interrupties kwamen overeen met die op de $\mu \mathrm{CT}$ voor beide afkapwaardes. Voor de volgende studies in dit proefschrift hebben we een diameter afkapwaarde van $\geq 0.410 \mathrm{~mm}$ gebruikt, aangezien we een hogere $P V W_{0.10 m m}$ prefereerden met de uitbreiding van het algoritme om trabeculair botverlies te detecteren.

Tot nu toe analyseerden we met ons algoritme alleen de aanwezigheid van een interruptie in de cortex van het bot en werd het onderliggend trabeculaire botverlies niet meegenomen als deel van het totale interruptievolume. Daarnaast was de reproduceerbaarheid van ons algoritme en de corticale microstructuur parameters op herhaalde scans met herpositionering tussen de scans nog niet getest. Daarom hebben we in hoofdstuk 5 ons corticale interruptie algoritme uitgebreid met detectie van verlies van onderliggend trabeculair bot. Verder hebben we de reproduceerbaarheid van het uitgebreide algoritme, de botdichtheid en microstructuur parameters van het corticale en trabeculaire gebied bestudeerd. Voor deze studie hebben we gebruik gemaakt van HR-pQCT beelden van de 'Early Inflammatory Arthritis Clinics' van de afdeling reumatologie op de universiteit van Calgary. Zij hebben 21 patiënten met een gemiddelde leeftijd van 49 (standaard deviatie (SD) 11 jaar) gerekruteerd waarvan 17 vroege RA en 4 ongedifferentieerde artritis, ieder minder dan 1 jaar geleden gediagnostiseerd.

De reproduceerbaarheid van het uitgebreide algoritme voor het aantal, oppervlak en volume van corticale interrupties was uitstekend (ICCs $\geq 0.82$ ), vooral voor het interruptievolume (ICC 0.99). De absolute minst significante verandering ( $\mathrm{MSV}_{\mathrm{SD}}$ ) was 4.2 voor het aantal interrupties, $5.8 \mathrm{~mm}^{2}$ voor het interruptieoppervlak en $3.2 \mathrm{~mm}^{3}$ voor het interruptievolume. De reproduceerbaarheid van de botdichtheid en bot microstructuur parameters was uitstekend met ICCS $\geq 0.97$, en was goed voor de corticale porie

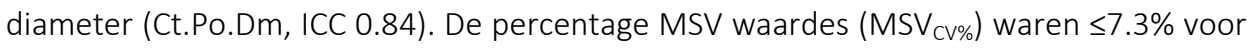
de botdichtheidsparameters en $\leq 12.1 \%$ voor de microstructuur parameters, behalve voor intra-individuele distributie van trabeculaire spreiding (Tb.SpSD), Ct.Po.Dm en corticale poreusheid (Ct.Po) welke tussen $21.1 \%$ en $24.1 \%$ waren. We concludeerden dat de reproduceerbaarheid van het algoritme goed was maar dat de precisie fout nog steeds substantieel is, terwijl de bepaling van de botdichtheid en bot microstructuur parameters goed reproduceerbaar waren met acceptabele precisie fouten.

Deze eerdere studies toonden de validiteit, betrouwbaarheid en toepasbaarheid van ons semiautomatisch algoritme in de detectie van (kleine) corticale interrupties. Het was echter nog steeds onbekend of deze interrupties gerelateerd zijn aan structurele schade 
op röntgenfoto's, en structurele schade en ontstekingsindicatoren op MRI. Daarnaast was het onbekend of HR-pQCT beeldvorming een toegevoegde waarde heeft over deze bevindingen op röntgenfoto's en MRI in de detectie van (kleine) corticale interrupties en in het onderscheidt tussen patiënten met RA en gezonde proefpersonen.

In hoofdstuk 6 hebben we daarom het aantal, oppervlak en volume van corticale interrupties in patiënten met RA vergeleken met gezonde proefpersonen door middel van ons semiautomatische HR-pQCT algoritme. Daarnaast hebben we de associatie tussen structurele schade op röntgenfoto's of MRI en ontstekingsindicatoren op MRI vergeleken met corticale interrupties op HR-pQCT. De toegevoegde waarde van de HRpQCT ten opzichte van de bevindingen op röntgenfoto's en MRI scans werd ook onderzocht. We hebben hiervoor gebruik gemaakt van het MOSA-Hand cohort dat bestaat uit 38 gezonde vrouwen en 39 vrouwen gediagnostiseerd met RA (gemiddelde leeftijd (SD) respectievelijk 51.2 (10.3) en 54.8 (7.0) jaar). ${ }^{36}$

Significant meer corticale interrupties en zowel een significant groter oppervlak als volume van corticale interrupties werden gedetecteerd in MCP gewrichten van patiënten met RA ten opzichte van gezonde proefpersonen. Bevindingen op röntgenfoto's en MRI scans waren significant geassocieerd met het aantal, oppervlak en volume van corticale interrupties op HR-pQCT (prevalentie ratio's (PRs) variërend van 1.03 tot 7.48 , allen $p<0.01$ ). Het hebben van RA was significant geassocieerd met het aantal, oppervlak en volume van corticale interrupties op HR-pQCT (PRs variërend van 2.26 tot 5.39 , allen $p<0.01$ ), ook na correctie voor bevindingen op röntgenfoto's en MRI scans. We concludeerden daarom dat HR-pQCT beeldvorming met het semiautomatische algoritme van waarde kan zijn naast de röntgenfoto's en MRI scans in de evaluatie van structurele botschade in patiënten met RA.

Cross-sectioneel discriminerend vermogen tussen patiënten met RA en gezonde proefpersonen is nu dus aangetoond met HR-pQCT, maar het longitudinale discriminerend vermogen was nog onbekend. Daarom hebben we in hoofdstuk 7 de longitudinale verandering na 1 jaar follow-up vergeleken tussen patiënten met RA en gezonde proefpersonen (MOSA-Hand cohort $^{36}$ ) in het aantal, oppervlak en volume van corticale interrupties, botdichtheid en bot microstructuur parameters. Elf van de 77 proefpersonen weigerden deelname aan de tweede visite en de gegevens van twee proefpersonen werden uitgesloten omdat ze geen HR-pQCT scans van voldoende kwaliteit hadden. In totaal konden 32 gezonde proefpersonen en 32 patiënten met RA worden onderzocht.

Het aantal, oppervlak en volume van interrupties was na 1 jaar niet veranderd op groepsniveau in zowel patiënten met RA als in gezonde proefpersonen, met uitzondering van een significante toename in het aantal interrupties in PIP gewrichten van patiënten met RA. De afname in botdichtheid was significant groter in MCP en PIP gewrichten van patiënten met RA ten opzichte van gezonde proefpersonen (respectievelijk -4.4 en -5.8 $\mathrm{mgHA} / \mathrm{cm}^{3}$ versus -1.1 en $-2.6 \mathrm{mgHA} / \mathrm{cm}^{3}$ ). De trabeculaire dikte nam ook significant meer af in PIP gewrichten van patiënten met RA ten opzichte van gezonde proefpersonen. 
Het toepassen van de MSV $_{S D}$ waardes liet zien dat de proportie van gewrichten die reparatie van het bot lieten zien hoger was in patiënten met RA ten opzichte van gezonde proefpersonen (respectievelijk 4.1\%, 3.6\% en $6.6 \%$ versus $0 \%$, $0 \%$ en $1.7 \%$ ) en het was significant voor het volume van interrupties (odds ratio (OR) (95\% betrouwbaarheidsinterval ( $\mathrm{BI}$ ) 1.08-39.9), $p=0.041$ ). De proportie van gewrichten die progressie in corticale interrupties toonden was niet significant verschillend tussen de groepen (respectievelijk $4.1 \%$, $2.7 \%$ en $5.9 \%$ versus $0.9 \%$, $0 \%$ en $2.6 \%$ ).

De proportie van gewrichten die progressie in corticale interrupties toonden was hoger in patiënten met 'synthetische disease modifying anti reuma drugs' (sDMARDs) vergeleken met patiënten behandeld met biologische DMARDs (bDMARDs) (respectievelijk $6.1 \%$, 3.0\% en $4.0 \%$ versus $1.8 \%$, 1.8\% en $7.9 \%$ ). Dit was significant voor het aantal interrupties (OR: 6.6 (95\% BI 1.0-41.7), $p=0.048$ ). De proportie van gewrichten met verlies van botdichtheid voorbij de MSV $_{S D}$ was significant hoger in patiënten behandeld met sDMARDs vergeleken met bDMARDs (respectievelijk 31.3\% versus 17.2\%, OR 2.7 (95\%BI 1.4-5.3), $p=0.004$ ).

We concludeerden dat HR-pQCT in staat is om botreparatie en botverlies te kwantificeren in vingergewrichten in patiënten met RA en gezonde proefpersonen. Corticale interruptie-, botdichtheid- en bot microstructuur parameters zijn aangedaan in patiënten met RA in vergelijking met gezonde proefpersonen, waarvan botdichtheid en bot microstructuur parameters verder verslechterde. Patiënten behandeld met sDMARDs lieten significant meer verlies in botdichtheid zien dat patiënten behandeld met bDMARDs. HR-pQCT met ons semiautomatisch algoritme liet veelbelovende resultaten zien in het monitoren van de effecten van behandeling in patiënten met RA.

Tot slot werden in hoofdstuk 9 de hoofdbevindingen van dit proefschrift samengevat en bediscussieerd in het kader van de door OMERACT vastgestelde termen "validiteit", "betrouwbaarheid", "discriminerend vermogen" en "toepasbaarheid" voor de detectie van corticale interrupties met het semiautomatische algoritme en de bepaling van de botdichtheid en bot microstructuur parameters.

Ten aanzien van "validiteit" bleek dat HR-pQCT sensitief was in de detectie van corticale interrupties in vergelijking met $\mu \mathrm{CT}$ (hoofdstuk 2). In hoofdstuk 3 zagen we dat het algoritme significant meer interrupties detecteerde dan met visuele scoring. Verschillen tussen visuele scoring en het semiautomatische algoritme waren voornamelijk gevonden door in regio's van lage corticale dichtheid, waar het lastig is om interrupties visueel te detecteren, terwijl het algoritme deze interrupties makkelijker kan detecteren op binaire beelden. Aan de andere kant wordt mogelijk de interruptie grootte overschat, door het partieel volume effect (PVE). In hoofdstuk 4, bleek dat de fout positieve detectie van corticale interrupties vermeden kon worden door de minimale diameter van interrupties te vergroten. In hoofdstuk 6, werd de validiteit van het semiautomatische algoritme getest ten opzichte van klinische beeldvormende methoden (röntgenfoto en MRI) en zagen we dat structurele schade op zowel röntgenfoto's als MRI scans geassocieerd waren met het aantal en de grootte van corticale interrupties op HR-pQCT. 
Ten aanzien van "betrouwbaarheid" bleek dat de betrouwbaarheid van het visueel scoren van corticale interrupties tussen 2 beoordelaars matig was. In hoofdstuk 3 was de betrouwbaarheid van het algoritme (zowel semiautomatisch als volautomatisch) in de detectie van corticale interrupties verbeterd was ten opzichte van visueel scoren (hoofdstuk 2). Echter de toepassing van het volautomatische algoritme resulteerden soms in fouten, voornamelijk in het geval van bewegingsartefacten en bij grote corticale interrupties. In hoofdstuk 5, vonden we dat de reproduceerbaarheid van het semiautomatische algoritme op herhaalde scans uitstekend was maar dat de precisie fouten toch nog substantieel waren. Over het algemeen vonden we een hogere betrouwbaarheid met het semiautomatische algoritme vergeleken met het visueel scoren van interrupties. De precisie fouten in onze studie waren vergelijkbaar met die van andere, alhoewel wij ook naar kleine corticale interrupties hebben gekeken in tegenstelling tot de andere studies. We hebben ook gekeken naar de betrouwbaarheid in de bepaling van de botdichtheid- en bot microstructuur parameters. We vonden een uitstekende intra- en inter-operator betrouwbaarheid evenals een uitstekende intraoperator reproduceerbaarheid, en deze waren vergelijkbaar met eerdere studies. Samenvattend konden we betrouwbaar corticale interrupties detecteren met het semiautomatische algoritme met een betrouwbare bepaling van de botdichtheid en bot microstructuur op HR-pQCT beelden van vingergewrichten.

Ten aanzien van het "discriminerend vermogen" vonden we meer en grotere corticale interrupties bij vroege RA patiënten (diagnose <2 jaar geleden) in vergelijking met gezonde proefpersonen (hoofdstuk 6). De aanwezigheid van RA was geassocieerd met meer en grotere interrupties ook na correctie voor structurele schade op röntgenfoto's en MRI scans en ontstekingsindicatoren op MRI scans. Na 1 jaar follow-up zagen we geen veranderingen in het aantal en grootte van interrupties in zowel patiënten met RA als in gezonde proefpersonen. Echter, op gewrichtsniveau zagen we zowel reparatie als progressie van botschade (interrupties) op HR-pQCT beelden, beide vooral in patiënten met RA. Verder hebben we het discriminerend vermogen van de botdichtheid en bot microstructuur onderzocht. We vonden een verminderde botdichtheid en bot microstructuur in patiënten met RA ten opzichte van gezonde proefpersonen. De botdichtheid en bot microstructuur verslechterden meer in patiënten met RA ten opzichte van gezonde proefpersonen na 1 jaar follow-up. De afname van botdichtheid was groter in patiënten behandeld met sDMARDs ten opzichte van bDMARDs.

Als laatste hebben we de "toepasbaarheid" van de HR-pQCT onderzocht. In eerder onderzoek rapporteerden we al dat visuele scoring niet uitvoerbaar is in een klinische setting omdat het erg tijdrovend is. Het toepassen van ons semiautomatisch algoritme kan mogelijk de evaluatie tijd verminderen. Correctie van de buitenste corticale contour bleek echter nog wel nodig, vooral in geval van bewegingsartefacten en grote corticale interrupties. Deze contour correctie is ook een standaard procedure voor HR-pQCT metingen van de distale radius en tibia. Waardoor na correctie van de buitencontour niet alleen het aantal en grootte van corticale interrupties, maar ook de botdichtheid en bot microstructuur automatisch kan worden bepaald. 
Concluderend, dit proefschrift laat zien dat semiautomatische analyse van HR-pQCT beelden een valide, betrouwbaar en toepasbaar instrument is voor detectie van corticale interrupties. Door toepassing van deze methode kan onderscheidt gemaakt worden tussen patiënten met RA en gezonde proefpersonen ten aanzien van de detectie van corticale interrupties (diameter $\geq 0.41 \mathrm{~mm}$ ), en bij bepaling van de botdichtheid en bot microstructuur in vingergewrichten. Daardoor kan HR-pQCT beeldvorming met ons algoritme bijdragen aan de evaluatie van peri-articulaire bot in vingergewrichten van patiënten met RA. 


\section{REFERENCES}

1. Schett G, Gravallese E. Bone erosion in rheumatoid arthritis: mechanisms, diagnosis and treatment. Nat Rev Rheumatol. 2012;8(11):656-64.

2. Geusens P, van den Bergh J. Bone erosions in rheumatoid arthritis. Rheumatology. 2014;53(1):4-5.

3. Myasoedova E, Crowson CS, Kremers HM, Therneau TM, Gabriel SE. Is the incidence of rheumatoid arthritis rising?: results from Olmsted County, Minnesota, 1955-2007. Arthritis Rheum. 2010;62(6): 1576-82.

4. Alamanos Y, Voulgari PV, Drosos AA. Incidence and prevalence of rheumatoid arthritis, based on the 1987 American College of Rheumatology criteria: a systematic review. Semin Arthritis Rheum. 2006;36(3):182-8.

5. van der Heijde DM. Radiographic imaging: the 'gold standard' for assessment of disease progression in rheumatoid arthritis. Rheumatology. 2000;39 Suppl 1:9-16.

6. Mottonen TT. Prediction of erosiveness and rate of development of new erosions in early rheumatoid arthritis. Ann Rheum Dis. 1988;47(8):648-53.

7. van der Heijde DM, van Leeuwen MA, van Riel PL, Koster AM, van 't Hof MA, van Rijswijk MH, et al. Biannual radiographic assessments of hands and feet in a three-year prospective followup of patients with early rheumatoid arthritis. Arthritis Rheum. 1992;35(1):26-34.

8. Nissila M, Isomaki H, Kaarela K, Kiviniemi P, Martio J, Sarna S. Prognosis of inflammatory joint diseases. A three-year follow-up study. Scand J Rheumatol. 1983;12(1):33-8.

9. Ravindran V, Rachapalli S. An overview of commonly used radiographic scoring methods in rheumatoid arthritis clinical trials. Clin Rheumatol. 2011;30(1):1-6.

10. Ostergaard M, Pedersen SJ, Dohn UM. Imaging in rheumatoid arthritis--status and recent advances for magnetic resonance imaging, ultrasonography, computed tomography and conventional radiography. Best practice \& research Clin Rheumatol. 2008;22(6):1019-44.

11. Baillet A, Gaujoux-Viala C, Mouterde G, Pham T, Tebib J, Saraux A, et al. Comparison of the efficacy of sonography, magnetic resonance imaging and conventional radiography for the detection of bone erosions in rheumatoid arthritis patients: a systematic review and meta-analysis. Rheumatology. 2011; 50(6):1137-47.

12. Dohn UM, Ejbjerg BJ, Court-Payen M, Hasselquist M, Narvestad E, Szkudlarek M, et al. Are bone erosions detected by magnetic resonance imaging and ultrasonography true erosions? A comparison with computed tomography in rheumatoid arthritis metacarpophalangeal joints. Arthritis Res Ther. 2006;8(4):R110.

13. Perry D, Stewart N, Benton N, Robinson E, Yeoman S, Crabbe J, et al. Detection of erosions in the rheumatoid hand; a comparative study of multidetector computerized tomography versus magnetic resonance scanning. J Rheumatol. 2005;32(2):256-67.

14. Szkudlarek M, Klarlund M, Narvestad E, Court-Payen M, Strandberg C, Jensen KE, et al. Ultrasonography of the metacarpophalangeal and proximal interphalangeal joints in rheumatoid arthritis: a comparison with magnetic resonance imaging, conventional radiography and clinical examination. Arthritis Res Ther. 2006;8(2):R52.

15. Conaghan PG, O'Connor P, McGonagle D, Astin P, Wakefield RJ, Gibbon WW, et al. Elucidation of the relationship between synovitis and bone damage: a randomized magnetic resonance imaging study of individual joints in patients with early rheumatoid arthritis. Arthritis Rheum. 2003;48(1):64-71.

16. Ostergaard M, Stoltenberg M, Lovgreen-Nielsen P, Volck B, Jensen CH, Lorenzen I. Magnetic resonance imaging-determined synovial membrane and joint effusion volumes in rheumatoid arthritis and osteoarthritis: comparison with the macroscopic and microscopic appearance of the synovium. Arthritis Rheum. 1997;40(10):1856-67.

17. Ostendorf B, Peters R, Dann P, Becker A, Scherer A, Wedekind F, et al. Magnetic resonance imaging and miniarthroscopy of metacarpophalangeal joints: sensitive detection of morphologic changes in rheumatoid arthritis. Arthritis Rheum. 2001;44(11):2492-502.

18. Boutroy S, Bouxsein ML, Munoz F, Delmas PD. In vivo assessment of trabecular bone microarchitecture by high-resolution peripheral quantitative computed tomography. J Clin Endocrinol Metab. 2005;90(12):6508-15. 
$172 \mid$ Addendum

19. Stach CM, Bauerle M, Englbrecht M, Kronke G, Engelke K, Manger B, et al. Periarticular bone structure in rheumatoid arthritis patients and healthy individuals assessed by high-resolution computed tomography. Arthritis Rheum. 2010;62(2):330-9.

20. Fouque-Aubert A, Boutroy S, Marotte H, Vilayphiou N, Bacchetta J, Miossec P, et al. Assessment of hand bone loss in rheumatoid arthritis by high-resolution peripheral quantitative CT. Ann Rheum Dis. 2010;69(9):1671-6.

21. Lee CH, Srikhum W, Burghardt AJ, Virayavanich W, Imboden JB, Link TM, et al. Correlation of structural abnormalities of the wrist and metacarpophalangeal joints evaluated by high-resolution peripheral quantitative computed tomography, 3 Tesla magnetic resonance imaging and conventional radiographs in rheumatoid arthritis. Int J Rheum Dis. 2015;18(6):628-39.

22. Finzel S, Ohrndorf S, Englbrecht M, Stach C, Messerschmidt J, Schett G, et al. A detailed comparative study of high-resolution ultrasound and micro-computed tomography for detection of arthritic bone erosions. Arthritis Rheum. 2011;63(5):1231-6.

23. Albrecht A, Finzel S, Englbrecht M, Rech J, Hueber A, Schlechtweg $P$, et al. The structural basis of MRI bone erosions: an assessment by microCT. Ann Rheum Dis. 2013;72(8):1351-7.

24. Binks DA, Gravallese EM, Bergin D, Hodgson RJ, Tan AL, Matzelle MM, et al. Role of vascular channels as a novel mechanism for subchondral bone damage at cruciate ligament entheses in osteoarthritis and inflammatory arthritis. Ann Rheum Dis. 2015;74(1):196-203.

25. Schett G, Stolina M, Bolon B, Middleton S, Adlam M, Brown H, et al. Analysis of the kinetics of osteoclastogenesis in arthritic rats. Arthritis Rheum. 2005;52(10):3192-201.

26. Regensburger A, Rech J, Englbrecht M, Finzel S, Kraus S, Hecht K, et al. A comparative analysis of magnetic resonance imaging and high-resolution peripheral quantitative computed tomography of the hand for the detection of erosion repair in rheumatoid arthritis. Rheumatology (Oxford). 2015; 54(9):1573-81.

27. Lee CH, Srikhum W, Burghardt AJ, Virayavanich W, Imboden JB, Link TM, et al. Correlation of structural abnormalities of the wrist and metacarpophalangeal joints evaluated by high-resolution peripheral quantitative computed tomography, 3 Tesla magnetic resonance imaging and conventional radiographs in rheumatoid arthritis. Int J Rheum Dis. 2015;18(6):628-39.

28. Finzel S, Rech J, Schmidt S, Engelke K, Englbrecht M, Stach C, et al. Repair of bone erosions in rheumatoid arthritis treated with tumour necrosis factor inhibitors is based on bone apposition at the base of the erosion. Ann Rheum Dis. 2011;70(9):1587-93.

29. Finzel S, Rech J, Schmidt S, Engelke K, Englbrecht M, Schett G. Interleukin-6 receptor blockade induces limited repair of bone erosions in rheumatoid arthritis: a micro CT study. Ann Rheum Dis. 2013;72(3):396-400.

30. Yue J, Griffith JF, Xiao F, Shi L, Wang D, Shen J, et al. Repair of bone erosion in rheumatoid arthritis by denosumab: A high-resolution peripheral quantitative computed tomography study. Arthritis Care Res. 2017;69(8):1156-63.

31. Srikhum W, Virayavanich W, Burghardt AJ, Yu A, Link TM, Imboden JB, et al. Quantitative and semiquantitative bone erosion assessment on high-resolution peripheral quantitative computed tomography in rheumatoid arthritis. J Rheumatol. 2013;40(4):408-16.

32. Zebaze R, Seeman E. Cortical bone: a challenging geography. J Bone Miner Res. 2015;30(1):24-9.

33. Boers M, Brooks P, Strand CV, Tugwell P. The OMERACT filter for Outcome Measures in Rheumatology. J Rheumatol. 1998;25(2):198-9.

34. Scharmga A. High-resolution peripheral quantitative computed tomography in patients with rheumatoid arthritis: truth, discrimination and feasibility. Thesis UM 2017.

35. Topfer D, Finzel S, Museyko O, Schett G, Engelke K. Segmentation and quantification of bone erosions in high-resolution peripheral quantitative computed tomography datasets of the metacarpophalangeal joints of patients with rheumatoid arthritis. Rheumatology. 2014;53(1):65-71.

36. Scharmga A, Peters M, van Tubergen A, van den Bergh J, Barnabe C, Finzel S, et al. Heterogeneity of Cortical Breaks in Hand Joints of Patients with Rheumatoid Arthritis and Healthy Controls Imaged by High-resolution Peripheral Quantitative Computed Tomography. J Rheumatol. 2016;43(10):1914-20. 


\section{DANKWOORD}

Eindelijk, na viereneenhalf jaar is mijn proefschrift helemaal klaar! Het was af en toe lastig, maar ik ben nu ook extra trots op het eindresultaat! Bij het tot stand komen van dit proefschrift zijn er natuurlijk veel mensen geweest die een steentje hebben bijgedragen. Hen wil ik dan ook graag bedanken.

Als eerste, bedank ik mijn promotie team voor hun vertrouwen in mij, hun expertise en de vele discussies over welke term nu toch het beste is voor een onderbreking van de cortex: erosie, cortical break, cortical discontinuity of cortical interruption.

Prof. dr. van den Bergh, beste Joop, hartelijk dank voor de begeleiding en het mede mogelijk maken van deze promotie plaats. Ik ben je erg dankbaar dat je aan mij dacht toen deze promotie plek vrij kwam. De meetings op de woensdag ochtend, vaak gezamenlijk met Piet, hebben me erg geholpen om ieder artikel goed uit te pluizen maar niet verzeilt te raken in de details, bedankt!

Prof. dr. Geusens, beste Piet, uw enthousiasme en kennis over onderzoek is erg aanstekelijk. Hierdoor kreeg ik na elke meeting weer een 'boost' om weer verder de literatuur of cijfers in te duiken. Door uw creatieve manier van denken had u op ieder (onverwacht) resultaat een goede verklaring, bedankt!

Dr. van Tubergen, beste Astrid, bedankt voor je input over de klinische invalshoek van dit onderwerp en de statistische ondersteuning. Verder heb je me erg geholpen door te hameren op de planning en het formuleren van concrete doelstelling voor artikelen, bedankt!

Dr. ir. van Rietbergen, beste Bert, tijdens mijn afstudeerstage onder begeleiding van jou en Chris vertelde ik al dat ik openstond voor een promotie traject, bedankt dat je aan mij hebt gedacht toen deze plek vrij kwam. Verder kan ik je rustige en nuchtere manier van werken erg waarderen en kon ik altijd bij je terecht voor de 'technische zaken', bedankt!

Verder wil ik de overige leden van de handenwerkgroep hartelijk danken voor hun input tijdens de maandelijkse meeting. René en Daan, dank voor jullie kritische houding ten opzichte van de klinische relevantie van mijn onderzoek. Verder was het erg prettig met jullie samenwerken in de zij-projecten waar we nog steeds gezamenlijk aan werken. Chris, tijdens mijn afstuderen heb je me erg geholpen met je dagelijkse begeleiding en de leuke trip naar Cork, hoewel nu meer op de achtergrond was je input nog erg waardevol, bedankt!

Other co-authors (Dr. K. Stok, Prof. S. Boyd, Dr. C. Barnabe and Dr. J. Driessen) and members of the SPECTRA collaboration thanks for the cooperation and valuable input. 
De leden van de beoordelingscommissie, Prof. dr. A. Boonen, Prof. dr. W. Backes, Prof. Dr. ir. K. Ito en Prof. dr. W. Lems wil ik bedanken voor het lezen en beoordelen van mijn proefschrift.

Verder wil ik de staf en medewerkers van de reumatologie bedanken voor alle hulp gedurende de afgelopen 4 jaar. Thea en Debby wil ik bedanken voor het scoren van de röntgenfoto's en Janine en Mehmet voor het mij leren scoren van de DAS28. Yvonne, hartelijk dank voor het helpen bij alle documentatie en het plannen van alle meetings door de jaren heen.

Leon Huiberts, wil ik bedanken voor de opslag van de anatomische vingers die ik altijd bij je kon brengen en ophalen.

En natuurlijk wil ik ook mijn mede promovendi bedanken. Carmen en Ivette, zoveel vrouwelijke 'roomies' was als ingenieur van de TU/e toch wel even wennen ;). Ellis, dank voor je enthousiasme en het initiatief nemen als organisator van de vele promovendi uitjes en barbecues! Andrea en Lieke, met jullie naar de verschillende congressen in het buitenland was erg gezellig! Joost, Simon, Bart, Casper en Frans, hartelijk dank voor de vele mannenavonden wat vooral bestond uit (veel) eten, bier en Fifa'en! Mayke, altijd leuk om gezamenlijk te fietsen, nu moet ik alleen nog een volledige toertocht fietsen! Gezamenlijk met Antje, José, Fiona, Caroline, Cindy, Yannick, en Lisanne hartelijk dank voor de leuke tijd als PhD student! De laatste maanden zijn Esther, Lennart, Mark en Ritch begonnen aan hun promotie, ik wens ze veel plezier en succes tijdens hun onderzoek!

Joost, Simon en Casper, als collega's zijn jullie goede vrienden van me geworden en wil ik jullie graag nog eens benoemen. Joost, als kamergenoot en mede ingenieur heb ik genoten van onze ontelbare avonden uit (vaak tot in de vroege uurtjes), de FIFA- en spellen avonden, en het wielrennen. Bedankt dat je altijd zin hebt om iets leuks te doen voor de afleiding! Casper, ik heb erg genoten van onze gezamenlijke congressen in Rome, Washington en Londen. Je adoratie ten opzichte van oud-werknemers van de Falstaff (quote: 'ze zijn half goden') blijft fascinerend! Simon, met jou had ik direct een klik tussen 'de vrouwen' van de reumatologie. Goede platte humor past ook bij jou. Gezamenlijk gingen we naar de fitness, maar vooral onze avond 'barbecueën' in een baby zwembadje was legendarisch! Erg jammer dat je er tijdens mijn verdediging niet bij kunt zijn.

Ook wil ik mijn studievrienden van de TU/e (Bob, Dennis, Jules, Paul, Rene, Tim) bedanken voor alle leuke dingen die we vaak ondernemen en de vele vakanties/ weekendjes weg door de jaren heen (Porto, Israël, Wie is de mol en Oktoberfeesten). Volgend jaar ga ik WIDM winnen! ;) Maarten en Luuk, bedankt dat ik jullie altijd mocht komen opzoeken (en de gratis slaapplek) als jullie je weer ergens anders op deze 
aardkloot bevonden! Vrienden uit Lierop, Luuk, Roel en het zaalvoetbal team, hartelijk dank voor de afleiding in de weekenden als ik weer in Lierop te vinden was.

Verder mag ik zeker 'ons pap en ons mam' niet vergeten. Waarschijnlijk zijn jullie nog trotser op dit resultaat als ik zelf ben. Ik kan bij jullie altijd terecht voor advies en steun, zowel persoonlijk als werk gerelateerd. Bedankt voor alles! Verder wil ik natuurlijk ook mijn broers, Mathijs (met Aukje) en Ruben (met Anouk) bedanken!

Last, maar zeker niet least, Marja. We kennen elkaar alweer ruim 3 jaar. Ik hoop dat we nog vele leuke dingen samen gaan ondernemen! Ik geniet er erg van als we samen gaan reizen, skiën, wandelen of wielrennen! Verder als mede-promovendi heb je me erg kunnen helpen tijdens mijn promotie, binnenkort is het jouw beurt! Ook bedankt dat je hand model wilde zijn voor de kaft van dit boekje! :; 
176 Addendum 


\section{CURRICULUM VITAE}

Michaël Petrus Henricus (Michiel) Peters werd geboren op 19 december 1988 in Lierop. $\mathrm{Na}$ het behalen van zijn VWO diploma aan het Varendonck college in 2007 met het profiel natuur en techniek begon hij aan de studie Biomedische Technologie aan de Technische Universiteit Eindhoven. Na afronding van zijn bachelor koos hij voor de master Medical Engineering. Tijdens zijn master heeft de focus gelegen op orthopedische biomechanica waarbij hij zijn afstudeerstage op de afdeling orthopedie bij het Maastricht Universitair Medisch Centrum (MUMC+) heeft uitgevoerd. Hier heeft hij zijn onderzoek gedaan naar de "performance" van zaagbladen bij knie vervangende operaties welke hij heeft mogen presenteren in een trip naar Cork in lerland en uiteindelijk tot een publicatie heeft geleidt. $\mathrm{Na}$ het afronden van zijn master begon hij aan zijn promotieonderzoek op de afdeling reumatologie in het MUMC+ in 2013 onder begeleiding van Prof. dr. J.P.W. van den Bergh, Prof. dr. P.P.M.M. Geusens, Dr. A. van Tubergen en Dr. ir. B. van Rietbergen. Resultaten van zijn onderzoek zijn gepubliceerd in verschillende internationale tijdschriften en resulteerden in dit proefschrift. Verder heeft hij zijn werk gepresenteerd op verschillende nationale en internationale congressen.

Tegenwoordig is Michiel aan het werk als klinisch informaticus op de afdeling radiologie van het MUMC+ onder begeleiding van dr. ir. R. Schnerr. 
178 Addendum 


\section{LIST OF PUBLICATIONS}

\section{This thesis}

- Scharmga A, Peters M, van Tubergen A, van den Bergh JP, de Jong JJ, Loeffen D, van Rietbergen B, Weijers R, Geusens P - Visual detection of cortical breaks in hand joints: reliability and validity of HR-pQCT compared to microCT. BMC Musculoskelet Disord. 2016 Jul 11;17:271.

- Peters M, Scharmga A, de Jong JJ, van Tubergen A, Geusens P, Arts JJ, Loeffen D, Weijers R, van Rietbergen B, van den Bergh JP - An Automated Algorithm for the detection of cortical interruptions on High Resolution peripheral Quantitative Computed Tomography Images of Finger Joints. PLoS One. 2017 Apr 20;12(4):e0175829.

- Peters M, Scharmga A, van Tubergen A, Arts JJ, Loeffen D, Weijers R, van Rietbergen $B$, Geusens P, van den Bergh JP - The Reliability of a semi-automated algorithm for detection of cortical interruptions in finger joints on High Resolution CT compared to microCT. Calcif Tissue Int. 2017 Aug;101(2):132-140.

- Peters M, de Jong JJ, Scharmga A, van Tubergen A, Geusens P, Loeffen D, Weijers R, Boyd SK, Barnabe C, Stok K, van Rietbergen B, van den Bergh JP - An automated algorithm for the detection of cortical interruptions and its underlying loss of trabecular bone; a reproducibility study. BMC Medical Imaging 201818:13

- Peters M, van Tubergen A, Scharmga A, Driessen JHM, van Rietbergen B, Loeffen D, Weijers R, Geusens P, Van den Bergh JP - Assessment of cortical interruptions with semi-automated analysis of HR-pQCT images in finger joints of patients with rheumatoid arthritis versus healthy subjects and in comparison to radiography and MRI. (JBMR 2018)

- Peters M, van den Bergh JP, Geusens P, Scharmga A, Loeffen D, Weijers R, van Rietbergen B, van Tubergen A - Prospective follow-up of cortical interruptions, bone density and micro-structure detected on high-resolution peripheral quantitative CT: a study in patients with RA and healthy subjects. (submitted) 


\section{Other publications:}

- Peters M, Feczko P, Tsang K, van Rietbergen B, Arts JJ, Emans PJ - Noise Exposure in TKA Surgery; Oscillating Tip Saw Systems vs Oscillating Blade Saw Systems. J Arthroplasty 2016;31(12):2773-2777

- Scharmga A, Keller KK, Peters M, van Tubergen A, van den Bergh JP, van Rietbergen B, Weijers R, Loeffen D, Hauge EM, Geusens P - Vascular channels in metacarpophalangeal joints: a comparative histologic and high resolution imaging study. Sci Rep. 2017 Aug 21;7(1):8966.

- Scharmga A, Peters M, van Tubergen A, van den Bergh J, Barnabe C, Finzel S, van Rietbergen B, Geusens P. - Heterogeneity of cortical breaks in hand joints of patients with rheumatoid arthritis and healthy controls imaged by HR-pQCT. J Rheumatol. 2016 Oct;43(10):1914-1920.

- Scharmga A, Geusens P, Peters M, van den Bergh JP, Loeffen D, Schoonbrood T, van Rietbergen B, Vosse D, Weijers R, van Tubergen A. - Structural damage and inflammation on radiographs or MRI are associated with cortical interruptions on high-resolution peripheral quantitative CT: a study in finger joints of patients with rheumatoid arthritis and healthy subjects. Scand J Rheumatol. 2018 Feb 20:1-9.

- Scharmga A, Peters M, A. van Tubergen, J. van den Bergh, J. de Jong, D. Loeffen, B. van Rietbergen, R. Weijers, P. Geusens - Cortical interruptions on High-Resolution peripheral Quantitative Computed Tomography in rheumatoid arthritis and healthy controls: development of a visual scoring method. (Submitted) 
\begin{tabular}{l|l} 
Publications & 181
\end{tabular} 
